\title{
MEASUREMENT OF THE DEUTERON ELASTIC STRUCTURE FUNCTIONS AT LARGE MOMENTUM TRANSFERS
}

\author{
by \\ Kathleen Rae McCormick \\ B.S. May 1993, Eastern Kentucky University \\ M.S. January 1995, University of Illinois at Urbana-Champaign \\ A Dissertation submitted to the Faculty of \\ Old Dominion University in Partial Fullfillment of the \\ Requirement for the Degree of \\ DOCTOR OF PHILOSOPHY \\ PHYSICS \\ OLD DOMINION UNIVERSITY \\ August 1999
}

Approved by:

Paul Ulmer (Director)

Gail Dodge

John Donat

Anatoly Radyushkin

Leposava Vušković 


\title{
ABSTRACT
}

\section{MEASUREMENT OF THE DEUTERON ELASTIC STRUCTURE FUNCTIONS AT LARGE MOMENTUM TRANSFERS}

\author{
Kathleen Rae McCormick \\ Old Dominion University, 2000 \\ Director: Dr. Paul Ulmer
}

The cross section for elastic electron-deuteron scattering has been measured using the Hall A Facility of Jefferson Laboratory. Scattered electrons and recoiling deuterons were detected in coincidence in the two $4 \mathrm{GeV} / \mathrm{c}$ High Resolution Spectrometers (HRS) of Hall A. The deuteron elastic structure functions $A\left(Q^{2}\right)$ and $B\left(Q^{2}\right)$ have been extracted from these data. Results for the measurement of $A\left(Q^{2}\right)$ in the range of $0.7 \leq Q^{2} \leq 6.0(\mathrm{GeV} / \mathrm{c})^{2}$ are reported. Results for the magnetic structure function, $B\left(Q^{2}\right)$, are presented in the range of $0.7 \leq Q^{2} \leq$ $1.35(\mathrm{GeV} / \mathrm{c})^{2}$. The results for both structure functions are compared to predictions of meson-nucleon based models, both with and without the inclusion of meson-exchange currents. The $A\left(Q^{2}\right)$ results are compared to predictions of the dimensional scaling quark model and perturbative quantum chromodynamics. The results can provide insights into the transition from meson-nucleon to quark-gluon descriptions of the nuclear two-body system. 


\section{(c) Copyright by Kathleen Rae McCormick 2000}

All Rights Reserved 
This work is dedicated to the memory of my Aunt Melissa January 9, 1962-May 20, 1996 


\section{Acknowledgments}

The first physics course I had was in Dayton High School. It was taught by Rosann Sharon. Her dynamic teaching influenced me to continue my physics studies in college. As an undergraduate at Eastern Kentucky University, I met many smart and witty people like Rosann. Not only did I get an excellent physics education from physicists like Charles Teague, Karl Kuhn, Dave Sousa, Jerry Cook and Chris Laird, but I also got a well rounded education in the Honors Program.

Thanks to Bonnie Gray I got the chance to travel to places I had only read about before. Thanks to Ron Messerich and Vonda Tolliver, I got to discuss scientific questions from a philosopher's viewpoint. From Ron and Eddy Thompson I also got my first exposure to many "classic" movies. Sean Cornett helped me to become a better scientist with hundreds of hours of discussion and table tennis, sometimes at the same time. We even discussed physics as we strolled through Europe on my first trip out of the U.S. From my friend Bruce MacLaren I learned that philosophy of science isn't as bad as it sounds, and I hope to be a thinker like he is some day. My home away from home while at Eastern was the home of Paula Kopacz and Andy Harnack. I want to thank them for the tons of spaghetti that they fed me and for the chance to discuss topics like the scientific methods used by the Puritan scientists.

I'd like to thank my friends at UIUC, especially Bart Terburg, who has given me tons of encouragement and friendship over the years. I also want to thank Dan Cronin and Jim Phillips for their continued friendship despite the distance that separated us when I left Illinois for Hampton Roads and Old Dominion University.

Here I've had the good fortune to have Paul Ulmer as my advisor. I always 
look forward to our conversations together and I will miss them when I'm gone. He taught me about what it means to actually do physics research and I'm very grateful for all of his help. Thanks to the other members of my committee: Gail Dodge, Ali Nowroozi, John Donat, Anatoly Radyushkin and Leposava Vuskovic for all of their suggestions on this work.

Most of my work has been done at Jefferson Lab (aka CEBAF). I want to thank the past and present Hall A staff: Larry Cardman, Eugene Chudakov, Ron Gilman, Ole Hansen, Kees De Jager, Meme Liang, John LeRose, Bob Michaels, Sirish Nanda, Eddy Offermann, Arun Saha and especially Bogdan Wojtsekhowski, J.P. Chen, Javier Gomez, Michael Kuss and Jack Segal for making CEBAF such a rewarding and fun place for me to work and learn. I want to thank Johnny Tang from the controls group for answering my EPICS questions any time of the day or night. I want to thank the other members of the E91-026 group for giving me such a pleasant working environment. Thanks to Riad Suleiman and Dave Prout for all of their hard work and late nights, to Makis Petratos for sitting down with me to go over presentations and to Mina Katramatou for her Monte Carlo. Without the Hall A technical staff, this experiment would never have been so successful. Thanks to Ed Folts, Mark Stevens, James Proffitt and Scot Spiegel for their patience when they were called in at 3:00 am. Thanks to the other Hall A graduate students and post-docs for all of their hard work. Many things in the Hall work today because of the efforts of Nilanga Liyanage, Krishni Wijesooriya, Juncai Gao, Kevin Fissum, Fraser Duncan, Bill Kahl, Christophe Jutier, Gary Rutledge, Riad Suleiman, Dave Prout, Alexandre Deur, Luminita Todor, Gilles Quemener and Mark Jones. Thanks to all of the other Hall A experimenters that took the time to sit shifts on this experiment.

I really want to thank my French friends. My life would have been totally different and much more boring if I hadn't met them, especially Eric Anciant, Sebastien Fabbro, Pascal Girard, David Pitz, Gerard Audit and Geraud Laveissiere, along with the honarary French Tony Day, Sasha Philips, Aziz Shafi, Paul Gueye, Gary Rutledge and Jochen Volmer.

Last but not least, I want to thank my family and especially my Aunt Missy. 


\section{Table of Contents}

List of Tables $\quad$ x

List of Figures $\quad$ xiii

1 INTRODUCTION 1

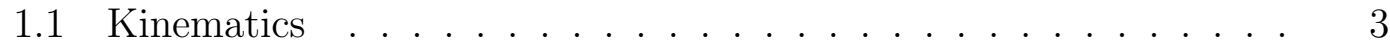

1.2 Deuteron Structure Functions and Form

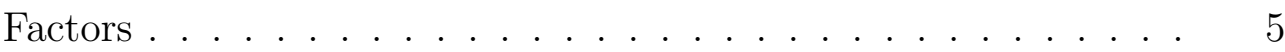

1.3 Experiment Overview . . . . . . . . . . . . 6

2 EXPERIMENTAL APPARATUS 11

2.1 Overview ............................. 11

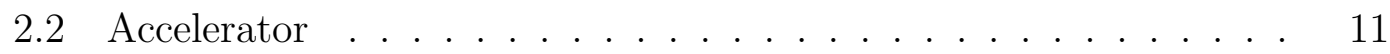

2.3 Hall A . . . . . . . . . . . . . . . . . . . . . . . . . . . 13

2.4 Hall A Beamline . . . . . . . . . . . . . . . . 16

2.4.1 Beam Position Monitors . . . . . . . . . . . . . 17

2.4.2 Beam Current Monitors . . . . . . . . . . . . . . . 18

2.4.3 Beam Rastering System . . . . . . . . . . . . . . 21

2.4.4 Beamline Magnetic Elements . . . . . . . . . . . . 21

2.4.5 Scattering Chamber . . . . . . . . . . . . . 23

2.4.6 Exit Beamline and Beam Dump ........... 24

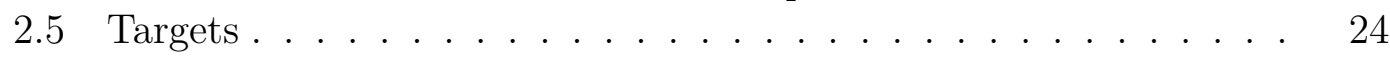

2.5.1 Cryotargets . . . . . . . . . . . . . . 24

2.5.2 Solid Targets . . . . . . . . . . . . . . . . . . . . 37

2.6 High Resolution Spectrometers . . . . . . . . . . . . . . 38

2.7 Detector Packages . . . . . . . . . . . . . . . . . 40

2.7.1 Vertical Drift Chambers . . . . . . . . . . . . . 41

2.7.2 Scintillator Hodoscope . . . . . . . . . . . . . . . 44

2.7.3 Čerenkov Detector ................ . . . 44

2.7.4 Pre-shower and Shower Detectors . . . . . . . . . . . . 46

2.8 Trigger Types and Electronics . . . . . . . . . . . . . . . 47 
2.9 Data Acquisition . . . . . . . . . . . . . . . . 50

2.9 .1 Coda Overview . . . . . . . . . . . . . . . . 50

2.9 .2 Coda Data File . . . . . . . . . . . . . . . . . . 52

3 DATA ANALYSIS 54

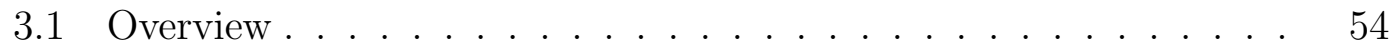

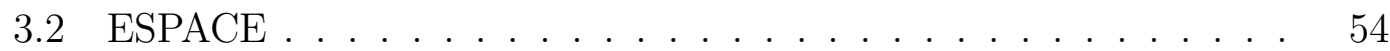

3.3 Spectrometer Optics Calibration . . . . . . . . . . . . . 55

3.4 Beam Energy Determination . . . . . . . . . . . . . . . . . 61

3.5 Total Charge Determination . . . . . . . . . . . . . . . 65

3.6 Electron and Recoil Tracking . . . . . . . . . . . . . 68

3.7 Electron Identification . . . . . . . . . . . . . . . . 70

3.7.1 Čerenkov Analysis . . . . . . . . . . . . . . . . . 70

3.7 .2 Shower Energy . . . . . . . . . . . . . . . . . . . 73

3.8 Recoil Particle Identification . . . . . . . . . . . . . . . 76

3.8.1 Coincidence Time-of-Flight . . . . . . . . . . . . . 77

3.9 Corrections to the Experimental Data . . . . . . . . . . 78

3.9.1 Detector and Trigger Inefficiencies . . . . . . . . . . . . 81

3.9.2 Deadtime and Prescaling Correction . . . . . . . . . 86

3.9.3 Recoil Nuclear Interactions . . . . . . . . . . . . . . . . . 87

3.9.4 Target Density Correction _. . . . . . . . . . . . . . 88

3.10 Monte Carlo Simulation . . . . . . . . . . . . . . . . . . . 89

3.11 Radiative Corrections . . . . . . . . . . . . . . . . . . . 94

3.12 Electron-Proton Elastic Cross Section
Calibrations . . . . . . . . . . . . . . . . . . . 95

4 RESULTS 101

$4.1 \quad A\left(Q^{2}\right)$ Measurement . . . . . . . . . . . . . . . . . . . 101

$4.2 B\left(Q^{2}\right)$ Measurement . . . . . . . . . . . . . . . . 110

5 COMPARISON WITH THEORY 114

5.1 Nonrelativistic Impulse Approximation . . . . . . . . . . . . . 114

5.2 Relativistic Impulse Approximation . . . . . . . . . . . . . . . 120

5.3 Meson Exchange Currents . . . . . . . . . . . . . . . . . . . . . 129

5.4 Quark Dimensional Scaling and Perturbative QCD . . . . . . 136

6 SUMMARY AND CONCLUSIONS 144

A Full Trigger Electronics Diagram 146

B Tables of Kinematics 150 
Bibliography

154

Vita

159 


\section{List of Tables}

I Cryotarget dimensions $(\mathrm{cm}) \ldots \ldots \ldots \ldots$

II Cryotarget parameters . . . . . . . . . . . . . . . . . . . 34

III Solid target thicknesses . . . . . . . . . . . . . . . . . . . 37

IV Properties of the lead glass used as the pre-shower (TF-1) and shower $($ SF5) detectors . . . . . . . . . . . . . . . . 47

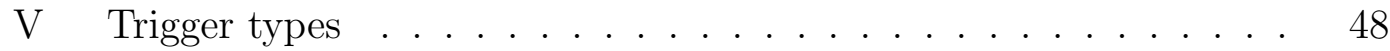

VI Trigger inefficiency corrections . . . . . . . . . . . . . 82

VII Ratio of the measured elastic e-p cross sections to theory for the $A\left(Q^{2}\right)$ Kinematics (collimators IN) . . . . . . . . . . . . 97

VIII Ratio of the measured elastic $e-p$ cross sections to theory for the $A\left(Q^{2}\right)$ Kinematics (collimators OUT) . . . . . . . . . 98

IX Ratio of measured elastic e-p cross sections to theory for the $B\left(Q^{2}\right)$ kinematics . . . . . . . . . . . . . . . . . . 98

X Measured cross sections for the 18 kinematical points taken for the $A\left(Q^{2}\right)$ extraction . . . . . . . . . . . . . . 102

XI Breakdown of $A\left(Q^{2}\right)$ systematic errors . . . . . . . . . . 103

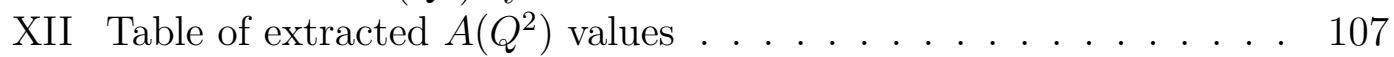

XIII Table of extracted $B\left(Q^{2}\right)$ values $\ldots \ldots \ldots \ldots \ldots$

XIV Deuteron static properties . . . . . . . . . . . . . . . 117

XV Kinematics for the $A\left(Q^{2}\right)$ measurements . . . . . . . . . 151

XVI Table of $\mathrm{H}\left(e, e^{\prime} p\right)$ kinematics for the $A\left(Q^{2}\right)$ measurements . . . . . 152

XVII Kinematics for the $B\left(Q^{2}\right)$ measurements . . . . . . . . . . . 152

XVIII Table of $\mathrm{H}\left(e, e^{\prime} p\right)$ kinematics for the $B\left(Q^{2}\right)$ measurements $\ldots 153$ 


\section{List of Figures}

1 Feynman diagram of elastic electron-deuteron scattering . . . . 4

2 Previous $A\left(Q^{2}\right)$ data in the range of $0.5 \leq Q^{2} \leq 1.9(\mathrm{GeV} / \mathrm{c})^{2} \ldots 8$

3 Previous $A\left(Q^{2}\right)$ data in the range of $0 \leq Q^{2} \leq 4.5(\mathrm{GeV} / \mathrm{c})^{2} \ldots 9$

4 Previous $B\left(Q^{2}\right)$ data in the range of $0 \leq Q^{2} \leq 3.0(\mathrm{GeV} / \mathrm{c})^{2} \ldots 10$

5 Schematic of the CEBAF Accelerator . . . . . . . . . . . 12

6 Schematic of Hall A . . . . . . . . . . . . . . . . . . . . 14

7 Aerial view of the inside of Hall A . . . . . . . . . . . . 15

8 The Hall A beamline from the shield wall through the $e-p$ energy measurement system (not to scale) . . . . . . . . . . . 16

9 The Hall A beamline from the Moller target to the cryotarget . . 17

10 Diagram of the Hall A current monitoring system . . . . . . . . 19

11 Beam profile with fast raster . . . . . . . . . . . . . . 22

12 Schematic of the Hall A cryotarget with attached solid target ladder . . . . . . . . . . . . . . . 25

13 Downstream view of the three cryotarget loops inside the scattering

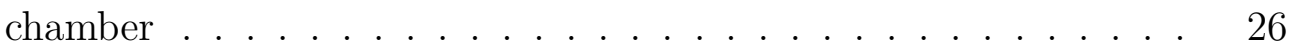

14 Diagram of a single target loop . . . . . . . . . . . 28

15 Side view of a target cell block . . . . . . . . . . . . . . . 29

16 Physical (left) and logical (right) architecture of a control system developed in the EPICS environment . . . . . . . . . . . 33

17 Normalized yield versus current for the $15 \mathrm{~cm} \mathrm{LH} 2$ target . . . . . 36

18 Normalized yield versus current for the $15 \mathrm{~cm}$ LD2 target . . . . . 36

19 Side view of a Hall A High Resolution Spectrometer . . . . . . . . 39

20 Diagram of the sieve slit collimator used on the Electron Arm. . . 40

21 Schematic of the Electron Arm detector package as used during this experiment . . . . . . . . . . . . . . . . . 4 41

22 Schematic of the Hadron Arm detector package as used during this experiment . . . . . . . . . . . . . . . . . 42

23 Schematic of the two Vertical Drift Chambers (VDCs) of the Electron Spectrometer . . . . . . . . . . . . . . . . . 43

24 Electron Arm $\mathrm{CO}_{2}$ gas Čerenkov detector . . . . . . . . . . . 45 
25 A simplified diagram of the HRSE, HRSH and Coincidence trigger circuits used for this experiment . . . . . . . . . . . . . . . . 49

26 Schematic of the Hall A DAQ . . . . . . . . . . . . . . 51

27 Hall A Laboratory Coordinate System (top view) . . . . . . . . . 56

28 Hall A Target Coordinate System . . . . . . . . . . . . . . . . 57

29 Hall A Detector Coordinate System . . . . . . . . . . . . . . . 59

30 Transport Rotated Coordinate System . . . . . . . . . . . . 60

31 Focal Plane Coordinate System . . . . . . . . . . . . . . . . . 60

32 Incident beam energy from $\mathrm{H}\left(e, e^{\prime} p\right)$ with nominal beam energy

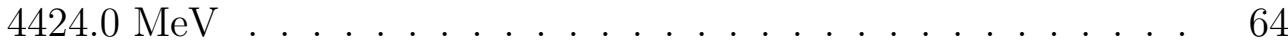

33 Incident beam energy versus run number (time) . . . . . . . . 66

34 Current calibration schematic . . . . . . . . . . . . 67

35 A typical trajectory in one of the VDC wire planes . . . . . . 69

36 ADC spectrum for a typical PMT on the EARM gas Cerenkov counter . . . . . . . . . . . . . . . . . 71

37 Sum of the ADC spectra for all ten phototubes of the Cerenkov

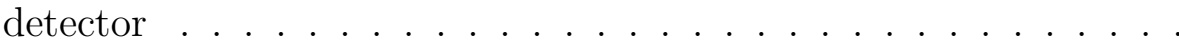

38 Cluster of blocks (shaded blocks) considered for the pre-shower (below) and shower (above) detectors for the given particle track (arrowed line) . . . . . . . . . . . . . . . . .

39 Raw spectrum of the ratio of the sum of the energy deposited in both the pre-shower and shower counters to the tracked momentum of the particle (EP Ratio) . . . . . . . . . . . . . . . .

40 Raw coincidence time (top) and corrected coincidence time (bottom) spectra from an $e-d$ run at $E_{\text {beam }}=3.245 \mathrm{GeV}$ and $Q^{2}=1.22$ $(\mathrm{GeV} / \mathrm{c})^{2} \ldots \ldots \ldots \ldots \ldots \ldots \ldots \ldots \ldots \ldots$

41 Corrected coincidence time spectrum with detector cuts applied . 80

42 Electron and Hadron ARM VDC efficiency . . . . . . . . . . . . 84

43 Electron arm Čerenkov inefficiency versus the scattered electron energy . . . . . . . . . . . . . . . . . 85

44 Shower counter inefficiency for backward (low energy) and forward (high energy) angle data . . . . . . . . . . . . . . . 86

45 Comparison of data to Monte Carlo for the electron spectrometer 91

46 Comparison of data to Monte Carlo for the hadron spectrometer . 92

47 Measured elastic $e-p$ cross sections divided by the Rosenbluth prediction using the form factors of Reference [39] . . . . . . . . . . 100

48 Previous $A\left(Q^{2}\right)$ and $B\left(Q^{2}\right)$ data to $4(\mathrm{GeV} / \mathrm{c})^{2} \ldots \ldots \ldots \ldots$

49 Fit to the existing world $B\left(Q^{2}\right)$ data $\ldots \ldots \ldots \ldots$

50 Hall A $A\left(Q^{2}\right)$ measurements to $Q^{2}=1.9(\mathrm{GeV} / \mathrm{c})^{2} \ldots \ldots \ldots$

51 Hall A $A\left(Q^{2}\right)$ measurements to $Q^{2}=6(\mathrm{GeV} / \mathrm{c})^{2} \ldots \ldots . . .109$ 
52 Hall A $B\left(Q^{2}\right)$ measurements to $Q^{2}=1.6(\mathrm{GeV} / \mathrm{c})^{2} \ldots \ldots \ldots 112$

$53 \quad B\left(Q^{2}\right)$ Measurements to $Q^{2}=4(\mathrm{GeV} / \mathrm{c})^{2} \ldots \ldots \ldots \ldots$

54 Feynman diagram for the Impulse Approximation . . . . . . . . 115

55 Comparison of the deuteron wave function between two NRIA models . . . . . . . . . . . . . . . . . . . . . . . . . . . . 118

56 NRIA comparison to $A\left(Q^{2}\right)$ data $\ldots \ldots \ldots \ldots . \ldots . \ldots 118$

57 NRIA comparison to $B\left(Q^{2}\right)$ data . . . . . . . . . . . . . 119

58 Schematic of relativistic approaches . . . . . . . . . . . . 122

59 A relativistic deuteron wave function . . . . . . . . . . . . 124

60 Feynman diagrams representing the Gross current matrix element 126

61 Comparison of several relativistic theories to the $A\left(Q^{2}\right)$ data . . 128

62 Comparison of several relativistic calculations to the $B\left(Q^{2}\right)$ data . 130

63 Feynman diagrams of Meson Exchange Currents . . . . . . . . . 132

64 Various models of the $\rho \pi \gamma$ MEC form factor . . . . . . . . . . . 133

65 Comparison of several relativistic calculations (with the inclusion of MEC) to the $A\left(Q^{2}\right)$ data . . . . . . . . . . . . . 134

66 Comparison of several relativistic calculations (with the inclusion of MEC) to the $B\left(Q^{2}\right)$ data . . . . . . . . . . . . . 137

67 Schematic of quark momentum exchange during elastic scattering 139

68 Deuteron form factor scaling . . . . . . . . . . . . . . . . . 141

69 The reduced deuteron form factor, $f_{d} \equiv \frac{F_{d}\left(Q^{2}\right)}{F_{N}^{2}\left(Q^{2} / 4\right)}$, as a function of

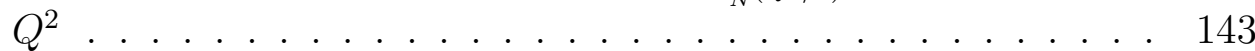

70 Electron Spectrometer Trigger Circuit . . . . . . . . . . . . . 147

71 Hadron Spectrometer Trigger Circuit . . . . . . . . . . . . . . . 148

72 Coincidence Trigger Circuit . . . . . . . . . . . . . . . . 149 


\section{Chapter 1}

\section{INTRODUCTION}

The nuclear force holds the nucleus together ${ }^{1}$. It acts between the nucleons in a nucleus, with a range that is of the same order of magnitude as the nucleon diameter. At moderate distance scales, the interaction between the nucleons is effectively described in terms of nucleons exchanging mesons. In this framework, the form of the nuclear potential is best studied by analyzing the interactions of two-body systems such as the deuteron, the only bound two nucleon system. The deuteron continues to be a useful "laboratory" at short distance scales also, such as those studied in elastic electron-deuteron scattering at high momentum transfers. There conventional meson-nucleon degrees of freedom may be insufficient to describe nuclear interactions and a description in terms of quark-gluon degrees of freedom may become more appropriate.

The deuteron is the bound state of a proton and a neutron. The ground state properties of the deuteron yield information about the character of the nuclear force. Some deuteron properties, such as its binding energy $(2.225 \mathrm{MeV})$, provide inputs which are used to constrain free parameters in nucleon-nucleon (NN) interaction models. Measurements of the deuteron magnetic and quadrupole moments have yielded $\mu=0.857406 \mu_{N}$ (where $\mu_{N}$ is the nuclear magneton) and $Q=0.2859$ $\mathrm{fm}^{2}$, respectively. The value of the quadrupole moment reveals information about

\footnotetext{
${ }^{1}$ The journal model for this thesis is Physical Review C.
} 
the deformation of the deuteron, which in turn reveals information about the nuclear tensor force. Since the deuteron is a relatively simple system, it is amenable to rigorous theoretical treatment and many such studies, using both relativistic and nonrelativistic approaches (see Chapter 5), abound in the literature.

Nonrelativistically, the Schrödinger equation can be solved with a high degree of accuracy for few nucleon systems and predictions for the deuteron static properties such as the $D / S$ ratio (where $D$ and $S$ denote the angular momentum states present in the deuteron wave function), the charge radius and the magnetic and quadrupole moments exist in all recent $N N$ interaction models [1]. In most cases, the models are able to provide accurate predictions for the static properties. The exceptions are the magnetic and quadrupole moments, which are underestimated in the nonrelativistic treatment, indicating that relativistic and two-body exchange corrections need to be taken into account even to describe the static properties of the deuteron. To study the electromagnetic structure of the deuteron, elastic electron scattering is employed.

In elastic electron-deuteron scattering, the electromagnetic structure of the deuteron is described in terms of three form factors which depend upon the square of the momentum transferred to the deuteron $\left(Q^{2}\right)$ during the interaction: the charge monopole, charge quadrupole and magnetic dipole, which are discussed in the sections that follow. These form factors can be determined via measurements of three observables. Two of these observables, the deuteron elastic electric and magnetic structure functions, known as $A\left(Q^{2}\right)$ and $B\left(Q^{2}\right)$ respectively, can be extracted from unpolarized elastic electron-deuteron scattering (the goal of this experiment). The third observable requires the use of a polarized beam and one such measurement $\left(T_{20}\right)$ is the focus of another JLAB experiment [2].

Many nonrelativistic predictions of the structure functions exist (cf. References [3] and [4]), but these predictions usually have to be augmented with corrections (such as relativistic corrections and meson exchange current (MEC) contributions) in order to describe the available data. Relativistically, the deuteron wave function can be derived from realistic $N N$ interaction models via solution of the Bethe-Salpeter and related quasipotential equations [5]. In the context of 
a relativistic model of the nuclear force, the deuteron structure functions can be calculated from only two contributions: the relativistic impulse approximation, in which the photon couples directly to one of the bound nucleons and the meson exchange current contribution in which the photon couples to the exchanged mesons. A variety of relativistic models have been used to calculate $A\left(Q^{2}\right)$ and $B\left(Q^{2}\right)$, but they tend to underestimate the data ( $c f$. Reference [6]). The failure of the relativistic theories to describe the data then gives an estimate of the contributions that meson exchange currents can make to the elastic $e$ - $d$ form factors $[5]$.

At sufficiently large momentum transfers, the deuteron form factors should be calculable in terms of only quarks and gluons within the framework of QCD. Perturbative QCD and dimensional counting rules predict the $Q^{2}$ dependence of the form factors, but the comparison with the available data seems to indicate that these calculations are not applicable below a momentum transfer squared of approximately $4(\mathrm{GeV} / \mathrm{c})^{2}[6]$.

This work describes a new set of measurements of the electric and magnetic structure functions $A\left(Q^{2}\right)$ and $B\left(Q^{2}\right)$. The next two sections of this chapter describe the kinematics of the reaction and the method of extracting the structure functions from the measured cross sections, while Section 1.3 gives an overview of the existing data and the physics goals of these new measurements.

\section{$1.1 \quad$ Kinematics}

The Feynman diagram for elastic electron-deuteron scattering in the one-photonexchange approximation is shown in Figure 1. This diagram represents an electron with energy $E$ incident on a stationary deuteron of mass $M_{d}$.

The four-momentum transfer squared is defined as

$$
Q^{2}=-q^{2}=4 E E^{\prime} \sin ^{2} \frac{\theta}{2}
$$




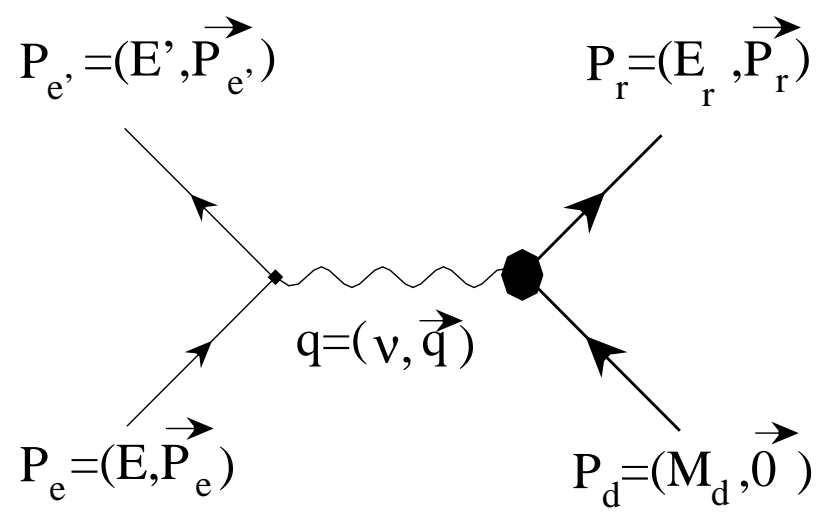

FIG. 1: Feynman diagram of elastic electron-deuteron scattering. The labels represent the four-momenta of the interacting particles and the exchanged photon.

where $E^{\prime}$ is the energy of the scattered electron and $\theta$ is the electron scattering angle. In the derivation of Equation (1), the electron rest mass has been neglected.

In a general scattering process, the invariant mass of the hadron in the final state is given by

$$
W^{2}=M^{2}+2 M \nu-Q^{2}
$$

where $\nu=E-E^{\prime}$ is the energy transfered to the target nucleus and $M$ is the mass of the target nucleus. For elastic scattering, $Q^{2}=2 M \nu$, so $W^{2}=M^{2}$ and the mass of the final state hadron is the same as that of the initial state.

The energy of the elastically scattered electron is given in terms of the energy of the incident electron, $E$, and the scattering angle as

$$
E^{\prime}=\frac{E}{1+\frac{2 E}{M_{d}} \sin ^{2} \frac{\theta}{2}} .
$$




\subsection{Deuteron Structure Functions and Form Factors}

The cross section for unpolarized elastic electron-deuteron scattering in the onephoton exchange approximation is described by the Rosenbluth formula,

$$
\frac{d \sigma}{d \Omega}=\sigma_{M}\left[A\left(Q^{2}\right)+B\left(Q^{2}\right) \tan ^{2}(\theta / 2)\right],
$$

where the Mott cross section, $\sigma_{M}$, describes the cross section for scattering from a structureless target:

$$
\sigma_{M}=\frac{\alpha^{2} E^{\prime} \cos ^{2} \frac{\theta}{2}}{4 E^{3} \sin ^{4} \frac{\theta}{2}}
$$

and the fine-structure constant $\alpha \cong 1 / 137$. The form of the Mott cross section shown in Equation (5) includes a factor to describe the recoil of the target nucleus. From Equation (4) we see that $A\left(Q^{2}\right)$ and $B\left(Q^{2}\right)$ can be separated by measuring the cross section at two different electron scattering angles while keeping the momentum transfer constant (Rosenbluth separation technique).

The structure functions $A\left(Q^{2}\right)$ and $B\left(Q^{2}\right)$ can be expressed in terms of the charge monopole $\left(F_{C}\right)$, charge quadrupole $\left(F_{Q}\right)$ and magnetic dipole $\left(F_{M}\right)$ form factors of the deuteron:

$$
\begin{gathered}
A\left(Q^{2}\right)=F_{C}^{2}\left(Q^{2}\right)+\frac{8}{9} \tau^{2} F_{Q}^{2}\left(Q^{2}\right)+\frac{2}{3} \tau F_{M}^{2}\left(Q^{2}\right), \\
B\left(Q^{2}\right)=\frac{4}{3} \tau(1+\tau) F_{M}^{2}\left(Q^{2}\right),
\end{gathered}
$$

where $\tau=Q^{2} / 4 M_{d}^{2}$. The kinematic factors multiplying $F_{Q}$ and $F_{M}$ are chosen so that in the static limit $\left(Q^{2}=0\right)$ 


$$
\begin{gathered}
F_{C}(0)=1, \\
F_{Q}(0)=M_{d}^{2} Q_{d}, \\
F_{M}(0)=\frac{M_{d}}{M_{p}} \mu_{d},
\end{gathered}
$$

where $F_{C}(0)$ is the electric charge of the deuteron, normalized to the elementary charge $e, Q_{d}$ is the deuteron quadrupole moment and $\mu_{d}$ is the deuteron magnetic dipole moment.

\subsection{Experiment Overview}

The first objective of this experiment was to obtain a precise measurement of the deuteron elastic electric structure function, $A\left(Q^{2}\right)$, from $Q^{2}=0.7$ to $6(\mathrm{GeV} / \mathrm{c})^{2}$. The measurement of $A\left(Q^{2}\right)$ at low momentum transfers (below $\left.2(\mathrm{GeV} / \mathrm{c})^{2}\right)$ was motivated by the apparent disagreement between the SLAC $A\left(Q^{2}\right)$ data [3] and the data from Saclay [7], CEA [8] and Bonn [9] in the region where they overlap, which is illustrated in Figure 2. Prior to this experiment, the deuteron $A\left(Q^{2}\right)$ structure function had been measured out to a maximum momentum transfer squared of $Q^{2}=4(\mathrm{GeV} / \mathrm{c})^{2}$ in SLAC Experiment E101 (Figure 3). Measurements at high momentum transfers probe the short distance structure of the deuteron, so it is at these momentum transfers that quark effects may become important. Therefore, our goal was to extend the measured $A\left(Q^{2}\right)$ range to a maximum of $Q^{2}=6(\mathrm{GeV} / \mathrm{c})^{2}$.

The second objective of this experiment was to provide a precise measurement of the deuteron elastic magnetic structure function, $B\left(Q^{2}\right)$, from $Q^{2}=0.7$ to 1.35 $(\mathrm{GeV} / \mathrm{c})^{2}$. As can be seen from Equation (7), a measurement of $B\left(Q^{2}\right)$ directly determines the deuteron magnetic dipole form factor, $F_{M}\left(Q^{2}\right)$. The magnetic structure function is difficult to measure above $Q^{2}=1.35(\mathrm{GeV} / \mathrm{c})^{2}$ with the current Hall A equipment because it falls very rapidly with momentum transfer, as illustrated in Figure 4 which shows the $B\left(Q^{2}\right)$ measurements made prior to this 
experiment. To extend the measurement of $B\left(Q^{2}\right)$ to higher momentum transfers in Hall A would require an additional electron detection system to be installed in the Hall to detect the scattered electrons at more backward angles then the current Hall A equipment allows [11].

This experiment, referred to as E91-026, took place in Hall A at Jefferson Laboratory (JLAB) in Newport News, Virginia. $A\left(Q^{2}\right)$ and $B\left(Q^{2}\right)$ were measured via elastic electron-deuteron $(e-d)$ scattering of the JLAB electron beam from a liquid deuterium target. The elastic $e-d$ data taking was divided into two periods. The first period was dedicated to measurements at backward electron scattering angles where the magnetic structure function's contribution to the cross section is maximized. Data were taken at $Q^{2}=0.7,0.83,0.96,1.09,1.22$ and $1.35(\mathrm{GeV} / \mathrm{c})^{2}$. The second period of data taking was dedicated to measuring the electric structure function, $A\left(Q^{2}\right)$, to the largest possible $Q^{2}$. Data were taken at $Q^{2}=0.7,0.83$, $0.96,1.09,1.22,1.35,1.80,2.4,3.5,4.0,4.5,5.0,5.4$, and $6.0(\mathrm{GeV} / \mathrm{c})^{2}$. Interspersed in the two month running period were elastic electron-proton cross section measurements which were used for calibration purposes. Tables of the kinematics for the $A\left(Q^{2}\right), B\left(Q^{2}\right)$ and $\mathrm{H}\left(e, e^{\prime} p\right)$ measurements can be found in Appendix B.

The experimental apparatus, including the JLAB accelerator and the Hall A equipment, are discussed in Chapter 2. The analysis method is discussed in Chapter 3. The results for $A\left(Q^{2}\right)$ and $B\left(Q^{2}\right)$ are given in Chapter 4. A discussion of several applicable theories and comparisons to the data are given in Chapter 5 . 


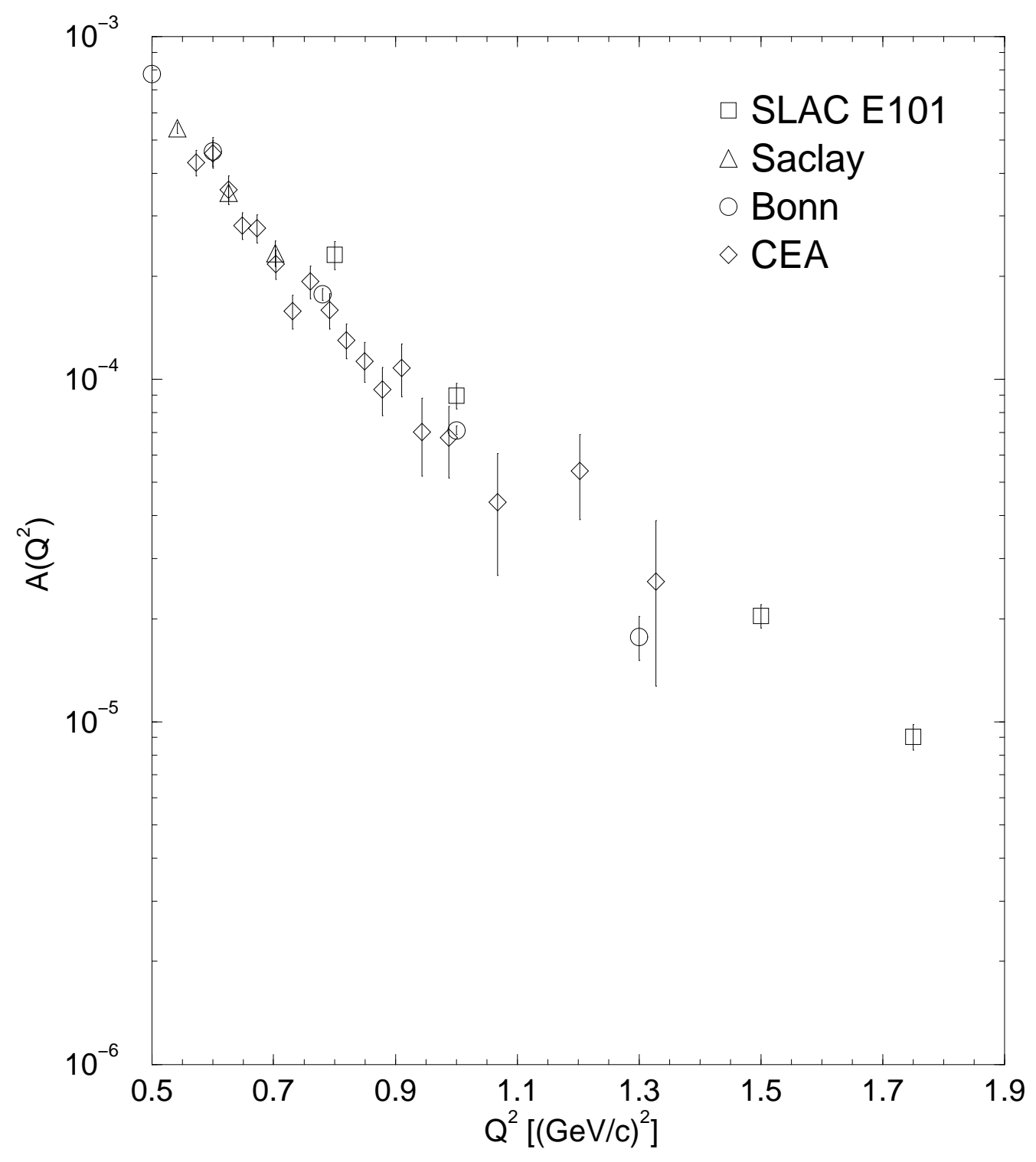

FIG. 2: Previous $A\left(Q^{2}\right)$ data in the range of $0.5 \leq Q^{2} \leq 1.9(\mathrm{GeV} / \mathrm{c})^{2}$. Shown are data from experiments performed at SLAC [3], Saclay [7], CEA [8] and Bonn [9]. 


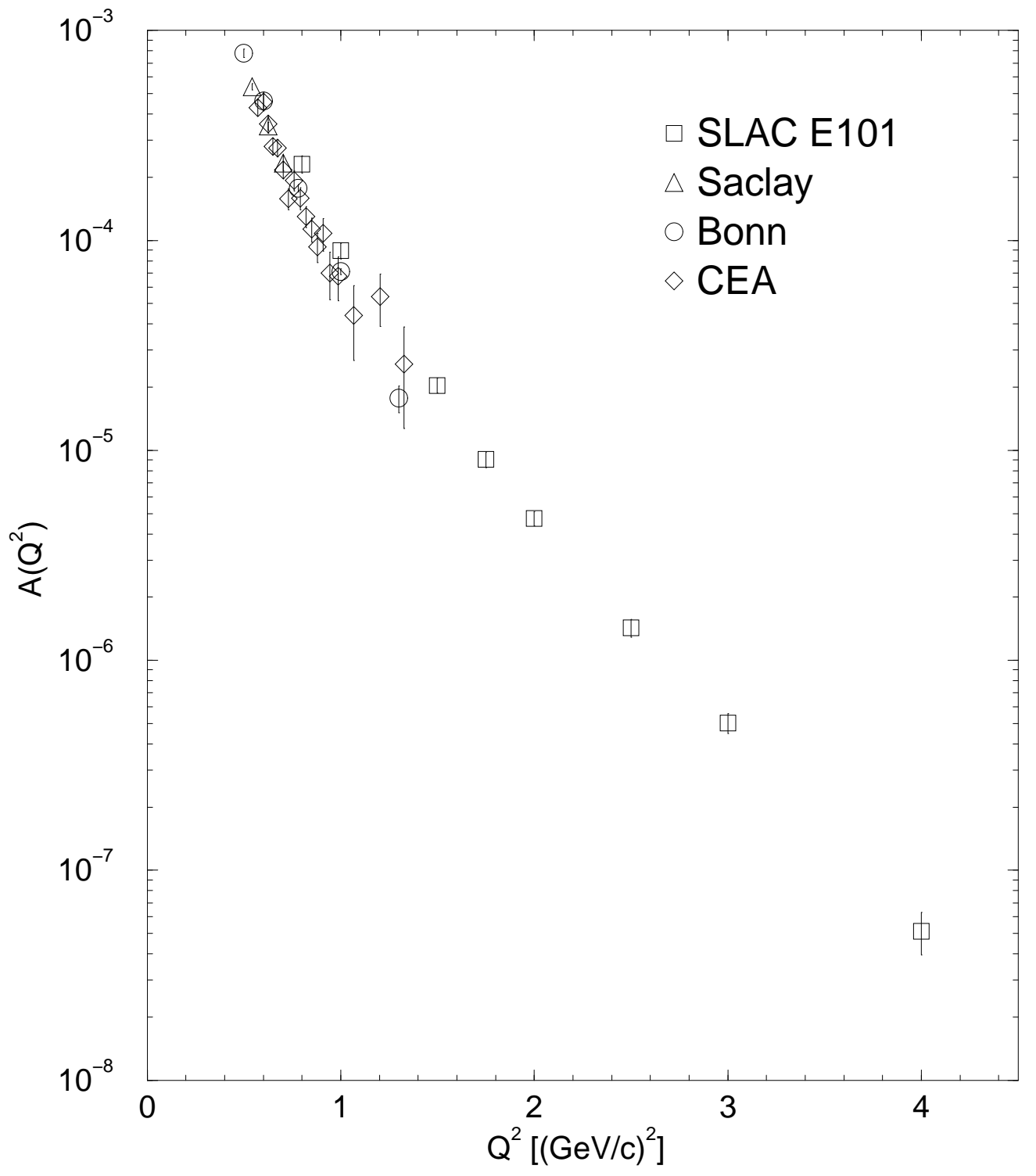

FIG. 3: Previous $A\left(Q^{2}\right)$ data in the range of $0 \leq Q^{2} \leq 4.5(\mathrm{GeV} / \mathrm{c})^{2}$. Shown are data from experiments performed at SLAC [3], Saclay [7], CEA [8] and Bonn [9]. 


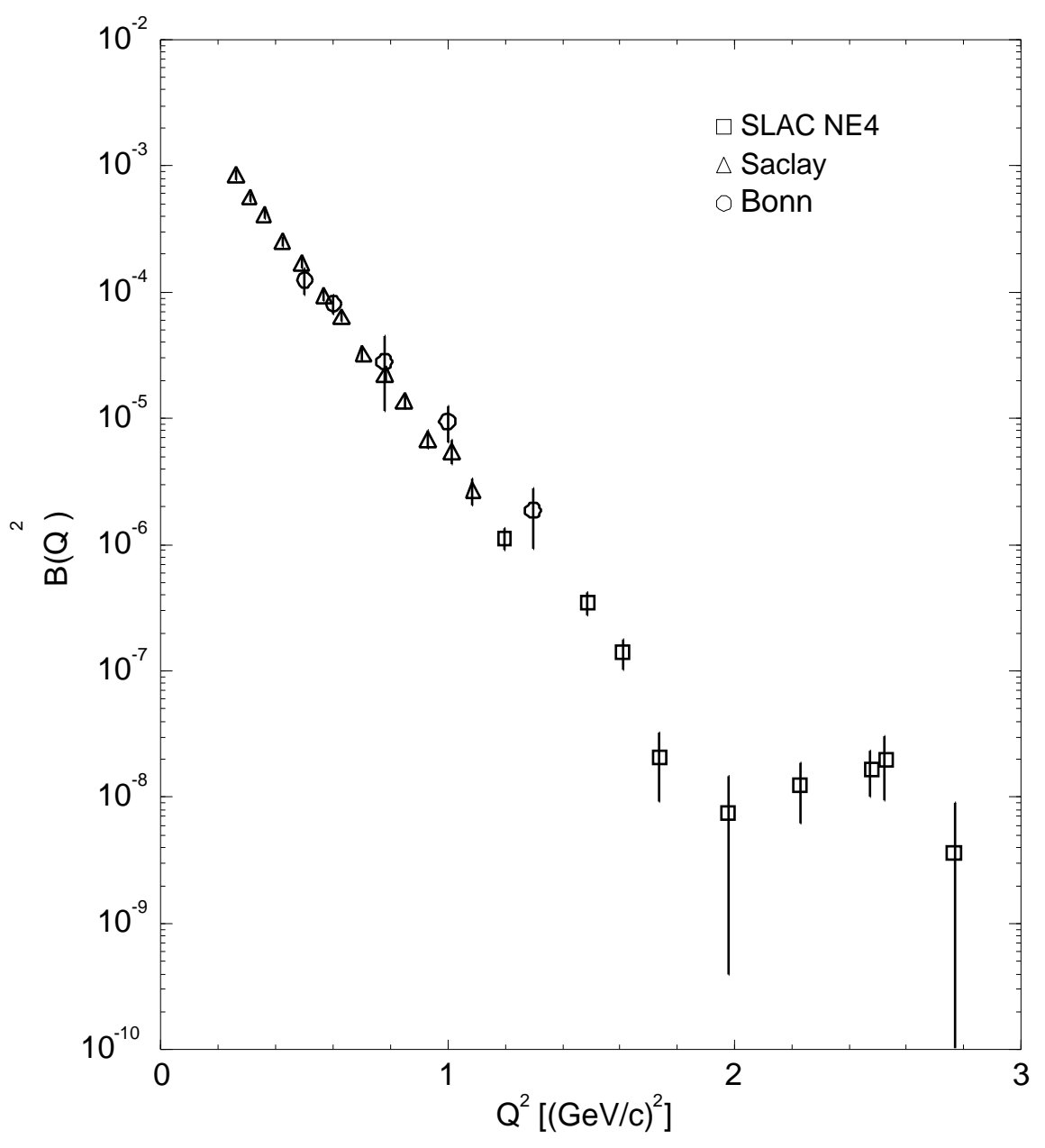

FIG. 4: Previous $B\left(Q^{2}\right)$ data in the range of $0 \leq Q^{2} \leq 3.0(\mathrm{GeV} / \mathrm{c})^{2}$. Shown are data from experiments performed at SLAC [4], Saclay [10], and Bonn [9]. 


\section{Chapter 2}

\section{EXPERIMENTAL APPARATUS}

\subsection{Overview}

Experiment 91-026, "Measurement of the Deuteron Elastic Structure Functions at Large Momentum Transfers", took place in experimental Hall A at Thomas Jefferson National Accelerator Facility. The data taking phase ran from October 13 through December 17, 1997, during which time the other two JLAB experimental halls, Hall B and Hall C, were also running experiments. The goal of E91-026 was to measure the elastic electric and magnetic structure functions of the deuteron via the $\mathrm{D}\left(e, e^{\prime} d\right)$ reaction. This was a coincidence experiment, with the scattered electron detected in the Electron Arm High Resolution Spectrometer (HRSE or EARM) and the recoil deuteron detected in the Hadron Arm High Resolution Spectrometer (HRSH or HARM). Calibration data from the $\mathrm{H}\left(e, e^{\prime} p\right)$ reaction were also taken. The data taking required a range of beam energies, from $0.6 \mathrm{GeV}-4.5 \mathrm{GeV}$, and beam currents, from $5-120 \mu \mathrm{A}$.

\subsection{Accelerator}

The JLAB accelerator was designed to deliver a continuous beam of electrons simultaneously to three experimental endstations. A diagram of the racetrack shaped accelerator is seen in Figure 5. The beam has a microstructure which 


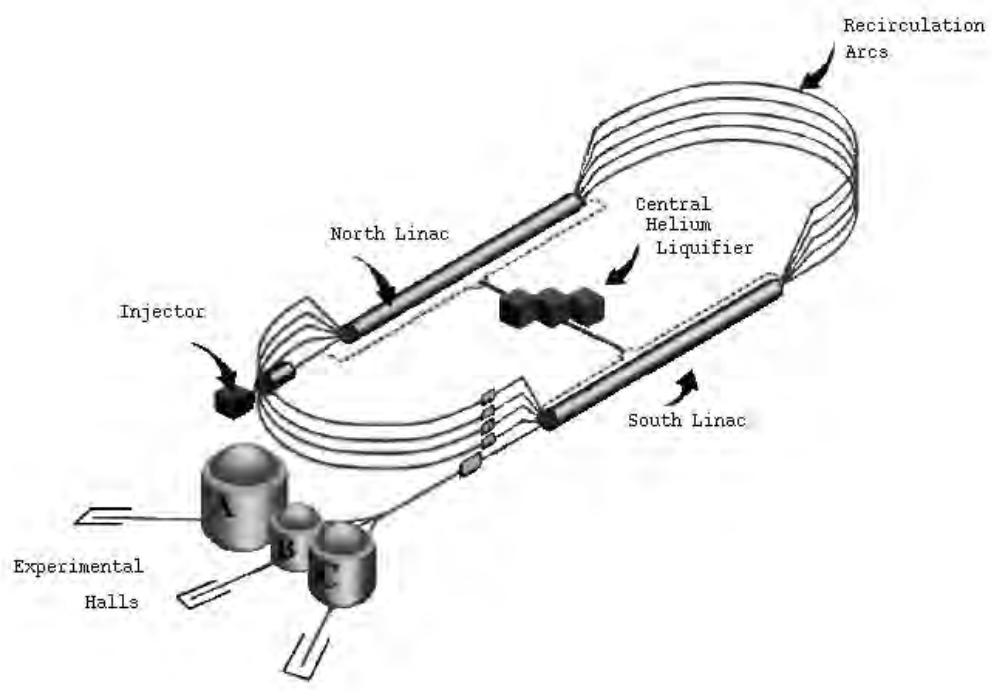

FIG. 5: Schematic of the CEBAF Accelerator.

begins at the injector. The injector produces electron beam pulses with an energy of $45 \mathrm{MeV}$ at a frequency of $1497 \mathrm{MHz}$. Next, the beam pulses are incident on a chopping aperture which contains slits of different sizes, one for each of the experimental Halls A, B and C. The widths of these slits determines the beam currents that are delivered to each hall. Each hall gets every third beam pulse directed through its slit, leading to a pulse train of $499 \mathrm{MHz}$ in the hall. After the slits, the beams for the three halls are injected into the North Linac.

The North Linac is a string of 20 cryomodules, with each cryomodule containing eight superconducting niobium cavities. These cavities are kept superconducting by helium coolant at a temperature of $2 \mathrm{~K}$ from the Central Helium Liquifier (CHL). Electric fields within the cavities accelerate the electrons as they travel down the Linac. At the end of the North Linac the electron beam has a nominal energy of $445 \mathrm{MeV}$ (45 MeV from the injector and $400 \mathrm{MeV}$ from the Linac), although by careful tuning of the accelerating electric field of the cavities, this energy can be raised or lowered, as was necessary several times during E91-026. 
Next, the beam enters the east magnetic recirculation arcs, where it is bent in a semi-circle to enter the South Linac. Here again the beam is accelerated through a string of 20 cryomodules. At the end of the South Linac the beam can be extracted for use in any of the the experimental halls or it can proceed around the west recirculation arcs for another pass around the accelerator. The beam can traverse the accelerator a maximum of five times, gaining a nominal $800 \mathrm{MeV}$ of energy with each pass around the machine. In the North and South Linacs the different energy beams resulting from each pass around the accelerator travel in the same beamline. However, the different energy beams require different bending fields in the recirculation arcs. When the beams reach the arcs they are separated by momentum and each go through a different arc (as seen in Figure 5). At the end of the arcs the beams are recombined into the same beamline again. When the beam is of the energy requested for a given experimental hall, it is extracted from the accelerator to the Beam Switch Yard (BSY). There every third beam pulse is sent into the appropriate experimental hall beamline by deflecting cavities operating at $499 \mathrm{MHz}$.

The nominal, unrastered diameter of the beam on target is approximately 200 $\mu \mathrm{m}(\mathrm{FWHM})$ at $845 \mathrm{MeV}$. The fractional energy spread $(\delta \mathrm{E} / \mathrm{E})$ is at the $10^{-4}$ level. The beam energy is known absolutely to $0.2 \%$ from the analysis of $\mathrm{H}\left(e, e^{\prime} p\right)$ scattering data taken in Hall A (see Section 3.4).

\section{$2.3 \quad$ Hall A}

Hall A is the largest of the three JLAB experimental halls, with a diameter of 53 meters. It contains two nominally identical high resolution magnetic spectrometers, respectively known as the Electron and the Hadron Spectrometers. Either spectrometer can be configured for positive or negative particle detection by changing the polarity of its magnetic elements. The spectrometers can be moved clockwise or counter-clockwise about the Hall A pivot where the target is located. The Electron spectrometer has a minimum central angle of $12.5^{\circ}$ with respect to the beamline and a maximum central angle of $144.5^{\circ}$, while the Hadron 


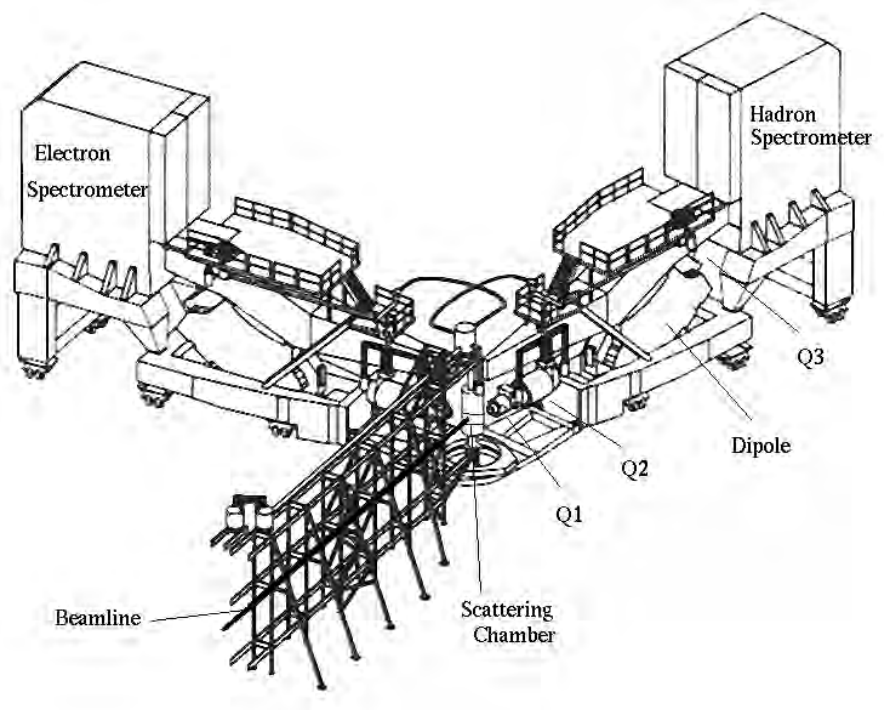

FIG. 6: Schematic of Hall A. Shown are the two high resolution spectrometers, the scattering chamber and the beamline.

spectrometer has a minimum central angle of $12.5^{\circ}$ and a maximum central angle of $130.5^{\circ}$.

A schematic of Hall $\mathrm{A}$ is shown in Figure 6. At the pivot point of the two spectrometers sits the cryotarget, encased within a cylindrical aluminum scattering chamber. The electron beam is incident on the target through an evacuated beamline. There are elements along the beamline for measurement of the beam current, beam position, beam energy and beam polarization. The primary target for this experiment was a high power liquid deuterium target. Scattered electrons and deuterons traverse the magnetic elements of the respective spectrometers and are detected by an array of detectors located in the shield house at the top of each spectrometer. Un-scattered electrons continue along the path of the beamline to the beamdump (not shown in Figure 6). A photograph of Hall A is shown in Figure 7. 


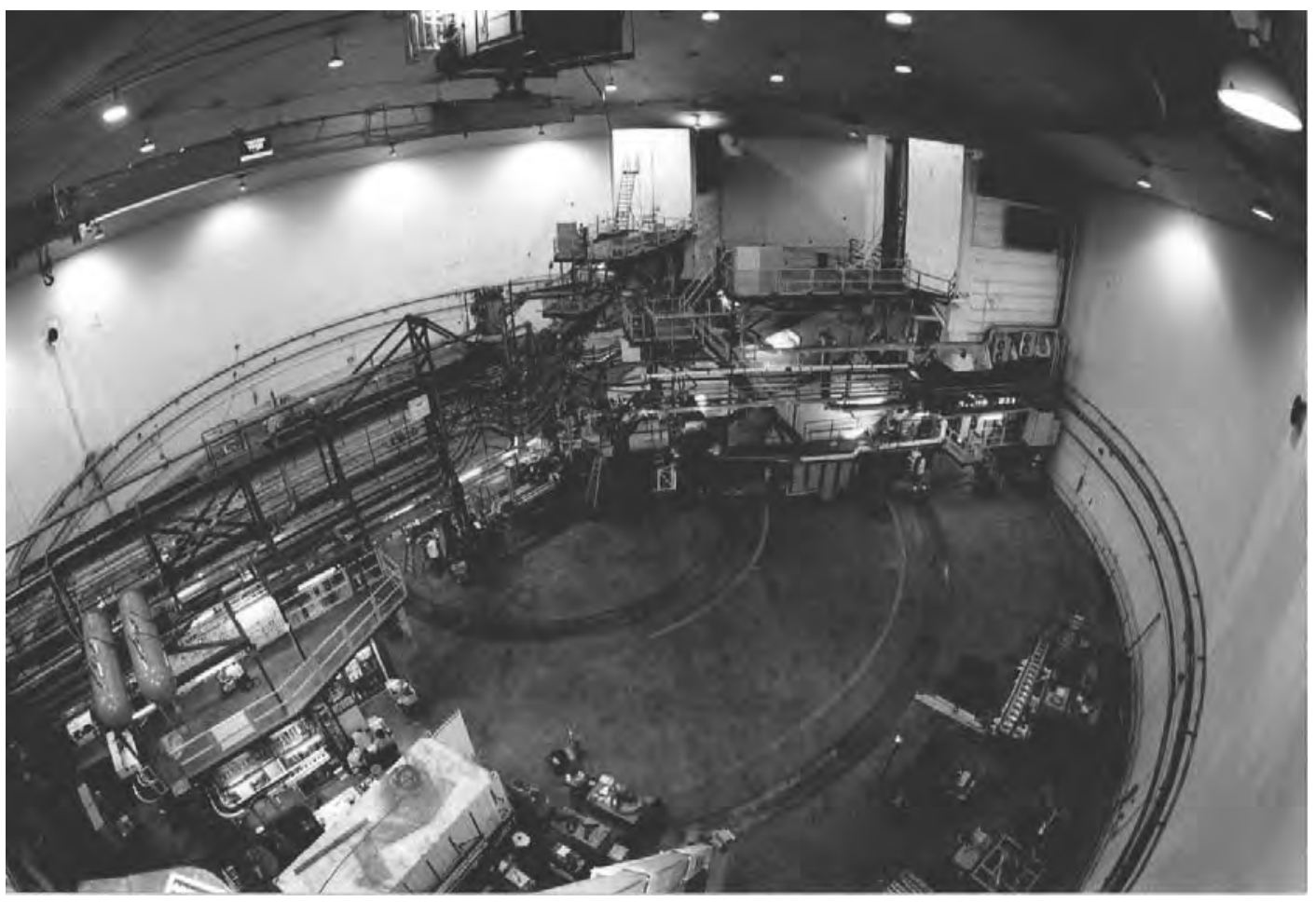

FIG. 7: Aerial view of the inside of Hall A. On the right are the two high resolution spectrometers. In this photo, the electron spectrometer is in the background, while the hadron spectrometer sits in the foreground. At the pivot point of the two spectrometers sits the cryotarget. To the left of the target is the beamline. In the foreground, various experimental electronics are visible. 


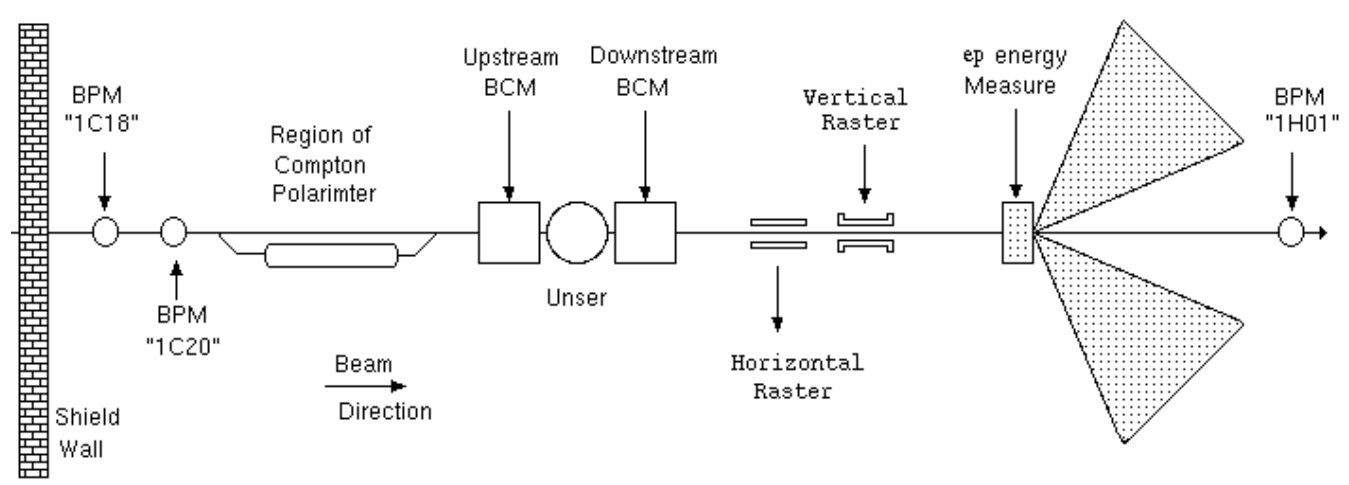

FIG. 8: The Hall A beamline from the shield wall through the $e-p$ energy measurement system (not to scale). This section of the beamline spans approximately 26 meters.

\subsection{Hall A Beamline}

When the beam has reached the requested energy, it is directed into the appropriate experimental hall. Beam bound for the Hall A target is bent into the hall through an arc containing eight magnets. Knowledge of the magnetic field strength of these magnets and the beam's path through them allows extraction of the incident beam energy. After entering the hall, the beam travels in a straight section of beamline until reaching the target. This straight section contains the major beamline elements, including the Compton polarimeter, the $e-p$ energy measurement device and the Moller polarimeter. None of these devices were directly employed during E91-026, although the magnetic elements of the Moller polarimeter were used for beam steering (see section 2.4.4 for more details). More details on all of these devices can be found on the Hall A web page [12]. The beamline also contains many smaller elements, such as the Beam Position Monitors (BPMs) used to monitor the beam position, the Beam Current Monitors (BCMs) which were used to measure the charge incident on the target and a beam rastering system which moved the beam around on target to prevent target density changes 


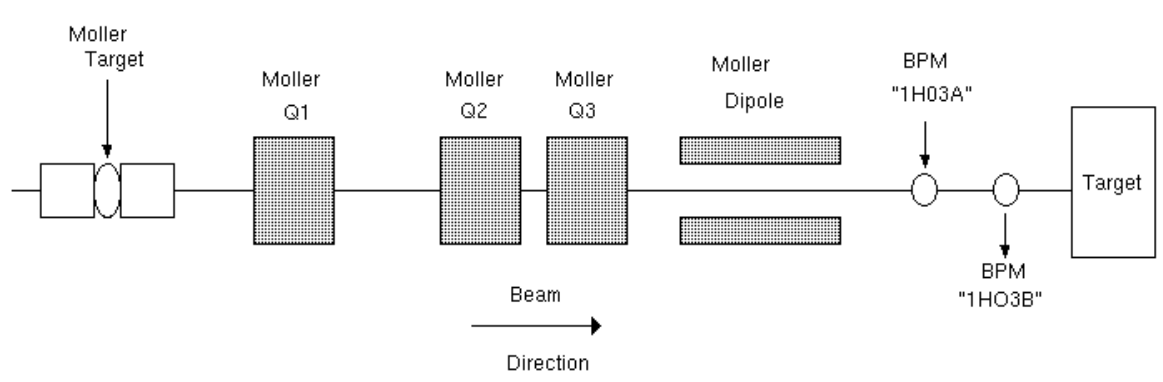

FIG. 9: The Hall A beamline from the Moller target to the cryotarget. This Figure is a continuation of Figure 8. This section of beamline spans approximately 18 meters. After the target, the un-scattered beam continues to the beam dump (not shown).

and damage to the target cell that would occur if the beam was concentrated on one spot.

\subsubsection{Beam Position Monitors}

There are five BPMs in the Hall A beamline between the target and the shield wall that separates Hall A from the accelerator (see Figures 8 and 9). These BPMs are used by the accelerator operators to monitor the position of the electron beam inside the beampipe. The BPMs measure the position of the beam non-destructively, and can therefore be used to continuously monitor the beam position.

Each BPM is a cavity with a four wire antenna array running parallel to its axis, with two wires being used to measure the $x$ (horizontal) position and the other two being used to measure the $y$ (vertical) position of the beam inside the cavity. The antenna-wires are positioned at $\pm 45^{\circ}$ from the horizontal and vertical. When the beam passes through the cavity, it induces a signal in each antenna wire, 
proportional to the distance from that wire. The $x$ and $y$ beam positions are then given by the asymmetry between the two $x$ antenna wire signals and the two $y$ antenna wire signals, respectively, multiplied by some calibration constants (for a complete description of the operation of the BPMs, consult [13]).

For the data taking phase of E91-026, the beam was steered so that its position was within $\pm 0.2 \mathrm{~mm}$ of nominal, as read on the last two BPMs before the target (denoted "1H03A" and "1H03B", respectively). The BPMs have been aligned relative to the center of the target cell via survey, with an uncertainty of approximately $0.5 \mathrm{~mm}$.

\subsubsection{Beam Current Monitors}

The beam current monitoring system for Hall A consists of a parametric current transformer (Unser monitor [14]) sandwiched between two resonant cavities [15]. The Unser monitor provides an absolute measurement of the current, but its offset drifts on the time scale of minutes, so it cannot be used to monitor the current continuously. Continuous monitoring of the current is accomplished with the resonant cavities. The cavities have a stable output signal that is proportional to the beam intensity, but they do not provide an absolute measure of the current. To get an absolute current measurement that is stable over time, the outputs of the resonant cavities are calibrated to that of the Unser monitor.

\section{Hardware}

A schematic of the current monitoring system is shown in Figure 10. In the upper right of this figure, we see the two resonant cavities, labeled Upstream and Downstream BCM, and the Unser monitor. The cavities are steel cylindrical waveguides, $15.48 \mathrm{~cm}$ in diameter and $15.24 \mathrm{~cm}$ long. Inside each cavity are two coaxial loop antennas. The smaller of the two loop antennas is for calibration purposes, while the larger picks up the signal from the beam. When the beam passes through a cavity, it excites the $\mathrm{TM}_{010}$ mode. This mode has a resonant frequency of $1497 \mathrm{MHz}$. The large loop antenna then provides an output signal 


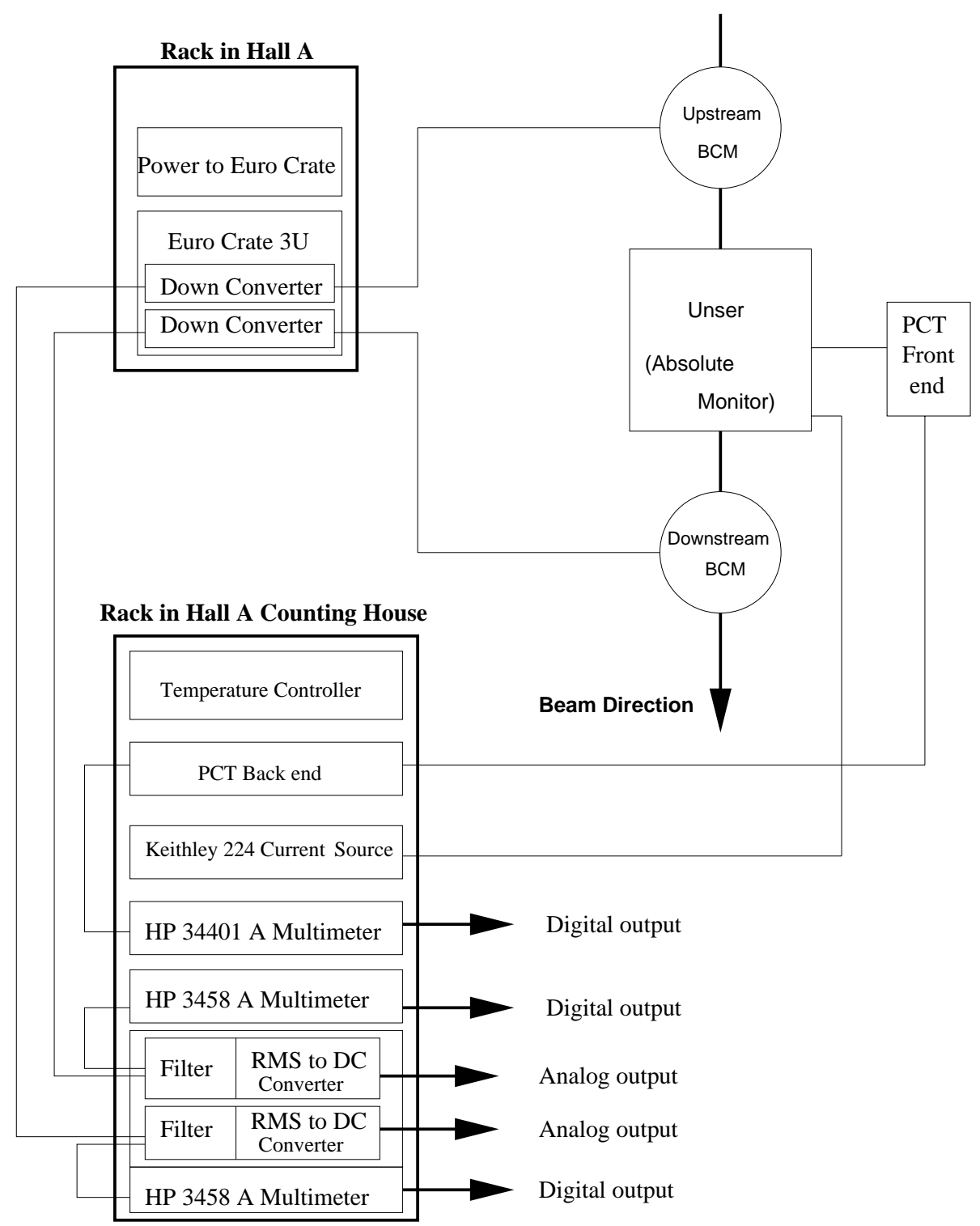

FIG. 10: Diagram of the Hall A current monitoring system. 
whose voltage is proportional to the beam current. Two possible sources of error in this type of current measurement are temperature variations and radial beam displacements. Since the resonant frequency of the cavity is sensitive to the dimension of the cavity (and therefore to temperature), the BCMs and Unser are housed in a temperature stabilized box regulated to $0.2{ }^{\circ} \mathrm{C}$. This variation leads to an error of $0.07 \%$ in the measured beam current. Also, the output signal of the cavity is sensitive to the radial displacement of the beam from the cavity axis, leading to an error of about $0.06 \%$ per $\mathrm{cm}$ of radial displacement. In practice, the beam is kept within millimeters of the cavity axis, leading to a very small error on the measured beam current from radial displacements.

The signal from each cavity is then fed into a down-converter (shown top-left in Figure 10), where it is reduced from $1497 \mathrm{MHz}$ to $1 \mathrm{MHz}$ for transmission to the electronics located in the Hall A counting house (bottom-left in Figure 10). For each BCM signal, two outputs are available in the counting house: a digital output from an HP 3458A multimeter and an analog output. The output from the digital multimeter is fed into a control system developed in the EPICS environment (see section 2.5.1 for more information about EPICS in general). This signal comes in one second intervals and represents an RMS average of the input signal during that second. The resulting number is proportional to the average beam current (and therefore charge) during that second. To get the absolute value of the beam current, the multimeter output is multiplied by a calibration constant (see section 3.5 for details on the measurement of the calibration constant). Roughly every four seconds, the value of the beam current (multimeter output $\times$ calibration constant) is written into the data stream, allowing later extraction of the charge accumulated during a data run.

The other output from the current measurement system is the analog output shown coming from the RMS to DC converters (one for each BCM) in the counting house (bottom-left in Figure 10). The RMS to DC converter produces a DC voltage level which is fed into a Voltage-to-Frequency (VTOF) converter whose output frequency is proportional to the input DC level. The output from the VTOF is then fed into scalers and injected into the data stream with the rest 
of the scaler information, leading to an accumulated scaler sum at the end of a run which is proportional to the beam charge. To get an absolute measure of the charge accumulated during a run, the scaler sum from the VTOF is compared to the EPICS output of the multimeter during that same run (for more details of the VTOF calibration, see [16]).

During this experiment, the beam delivery was stable, so the charge accumulated during a run was extracted from the (sampled) multimeter data which was written into the data stream.

\subsubsection{Beam Rastering System}

In an effort to prevent target density changes and damage from depositing too much beam power in too small an area, the beam is rastered before it interacts with the target. The hardware for the rastering system is located 23 meters before the cryotarget, between the beam current monitors and the $e$ - $p$ energy measurement system ( $c f$. Figure 8). For a complete description of the raster hardware, see Reference [17].

The fast rastering system consists of two sets of steering magnets. The first set has its magnetic field oriented so as to deflect the beam horizontally and the second set has its magnetic field oriented to deflect the beam vertically. The magnetic fields of the deflecting coils were varied sinusoidally, at $25.3 \mathrm{kHz}$ in the horizontal direction and $17.7 \mathrm{kHz}$ in the vertical direction. The ratio of the oscillation frequencies of the two coils was chosen so that the resulting raster pattern would sweep out a rectangular pattern at the target. The beam position on an event by event basis is shown in Figure 11. Since the beam rastering was sinusoidal, the beam spent more time near the edges of the raster pattern.

\subsubsection{Beamline Magnetic Elements}

As seen in Figures 8 and 9, the Moller polarimeter sits between the $e$-p energy measurement system and the cryotarget. The Moller polarimeter is used to make 


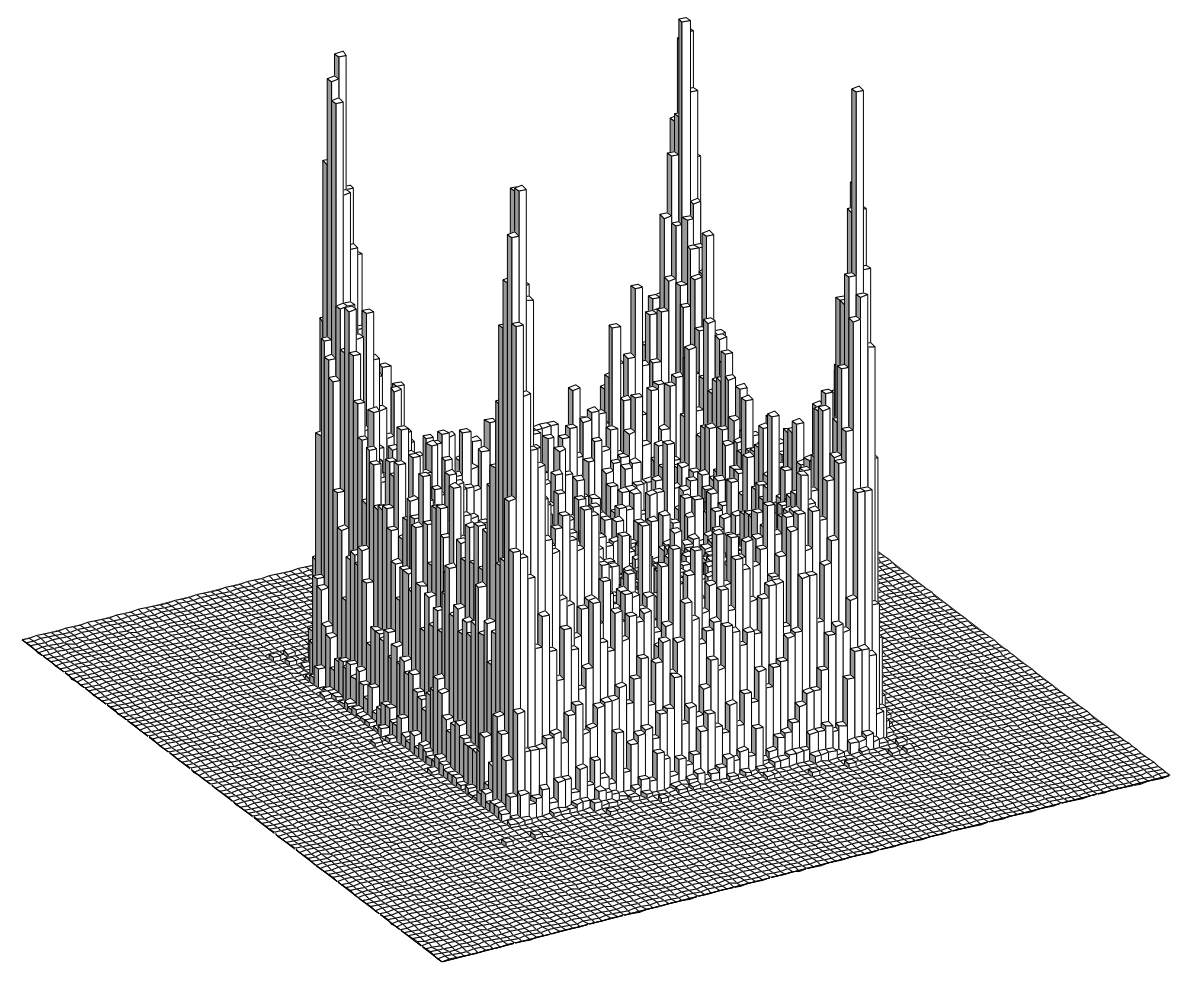

FIG. 11: Beam profile with fast raster. This plot shows the position of the beam on target, taken on an event by event basis. For normal data taking, the raster size was set for $\pm 1.7 \times \pm 1.4 \mathrm{~mm}$ ( $x$ and $y$ dimensions, respectively), while the spot in this picture is approximately $\pm 2.5 \times \pm 2.5 \mathrm{~mm}$. 
measurements of the incident electron beam's polarization. The beam was unpolarized for this experiment, so no Moller measurements were made, but the three quadrupole magnetic elements of the Moller polarimeter were used by the machine operators for general beam steering.

\subsubsection{Scattering Chamber}

The scattering chamber used during this experiment consisted of three sections. The bottom section was fixed to the pivot of the Hall. This section contained several viewports through which the targets could be visually inspected and several ports for vacuum pumps. The middle section where the beam interacted with the target was an aluminum cylinder with an inner diameter of $104 \mathrm{~cm}$, a height of $91 \mathrm{~cm}$ and a wall thickness of 5 centimeters. The beam entrance and exit pipes were coupled directly to this central section, so the beam passed through no material before interacting with the target. Scattered particles exited the scattering chamber through exit windows. Both exit windows were $18 \mathrm{~cm}$ tall and together these windows spanned about $93 \%$ of the scattering chamber's circumference, interrupted only by supports for the beam entry and exit and four additional smaller supports spaced around the circumference. The scattering chamber exit windows for both the electron and hadron spectrometer were made of $0.4064 \mathrm{~mm}$ thick Aluminum. The third and uppermost section of the scattering chamber contained space for the cryogenic target plumbing.

All three sections of the scattering chamber were maintained under vacuum. Besides reducing multiple scattering, the chamber vacuum served as an insulator which helped keep the cryogenic target cold. The vacuum level was carefully maintained at the $10^{-6}$ Torr level. Any degradation in the scattering chamber vacuum was quickly noted, as it led to an increase in temperature of the cryogenic target. 


\subsubsection{Exit Beamline and Beam Dump}

Attached directly to the rear of the scattering chamber is the exit beamline, which terminates at the Hall A beam dump. The exit beamline is divided into two sections which are separated by a kapton window. In the first section after the scattering chamber (approximately $1.5 \mathrm{~m}$ long), the vacuum is maintained at the same level as in the primary beamline $\left(10^{-6}\right.$ Torr $)$. In the second section, after the kapton window, the vacuum is maintained at the level of $10^{-4}$ Torr. This second section extends the remaining approximately 27.5 meters to the beam dump. The beam terminates on the beam dump. The window of the Hall A beam dump is a sandwich of two beryllium plates with water flowing between them. After this diffuser, beam particles continue on to the main beam dump through a helium filled pipe approximately $45 \mathrm{~cm}$ in diameter. The main beam dump contains water-cooled aluminum plates where the beam can deposit its energy.

\subsection{Targets}

A schematic of the target ladder used in this experiment is shown in Figure 12. Shown in this figure (from top to bottom) are the three cryogenic target loops, the three aluminum dummy targets that were used to estimate the contribution from the target cell windows and the five solid targets that were used for various calibration purposes.

\subsubsection{Cryotargets}

\section{Hardware}

The cryogenic portion of the Hall A target consists of three target loops, each of which has two target cells (see Figure 13). These target cells are of lengths 15 $\mathrm{cm}$ and $4 \mathrm{~cm}$. The cryogen in Loop 2 is liquid hydrogen, while the cryogen in Loops 1 and 3 can alternate between liquid deuterium and gaseous ${ }^{4} \mathrm{He}$ according to data taking requirements. During the first period of E91-026, data was taken for the $B\left(Q^{2}\right)$ measurement, using the $15 \mathrm{~cm}$ Loop 1 target cell as the deuterium 


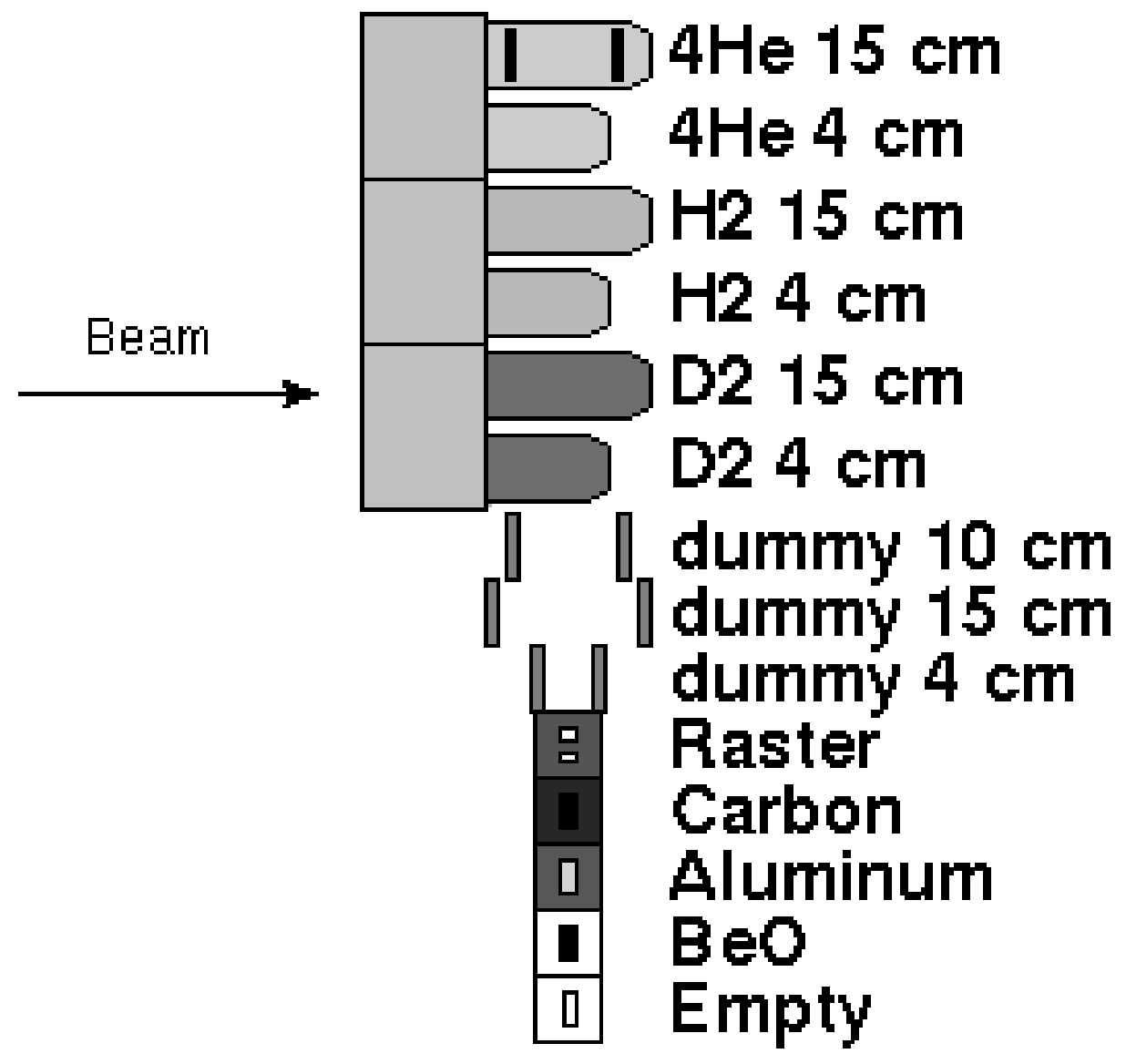

FIG. 12: Schematic of the Hall A cryotarget with attached solid target ladder. Shown, from top to bottom, are the three cryogenic target loops (each loop consists of two cells of lengths 15 and 4 centimeters), the three aluminum dummy targets and the five solid targets. The black lines on the $15 \mathrm{~cm}$ Loop 1 target cell represent a collimator that was installed to remove electrons from the acceptance that would re-scatter from the target cell block (the aluminum block to which the target cells are attached). The cryogen in Loop 1 alternated between liquid deuterium or gaseous helium, depending upon data taking requirements. 


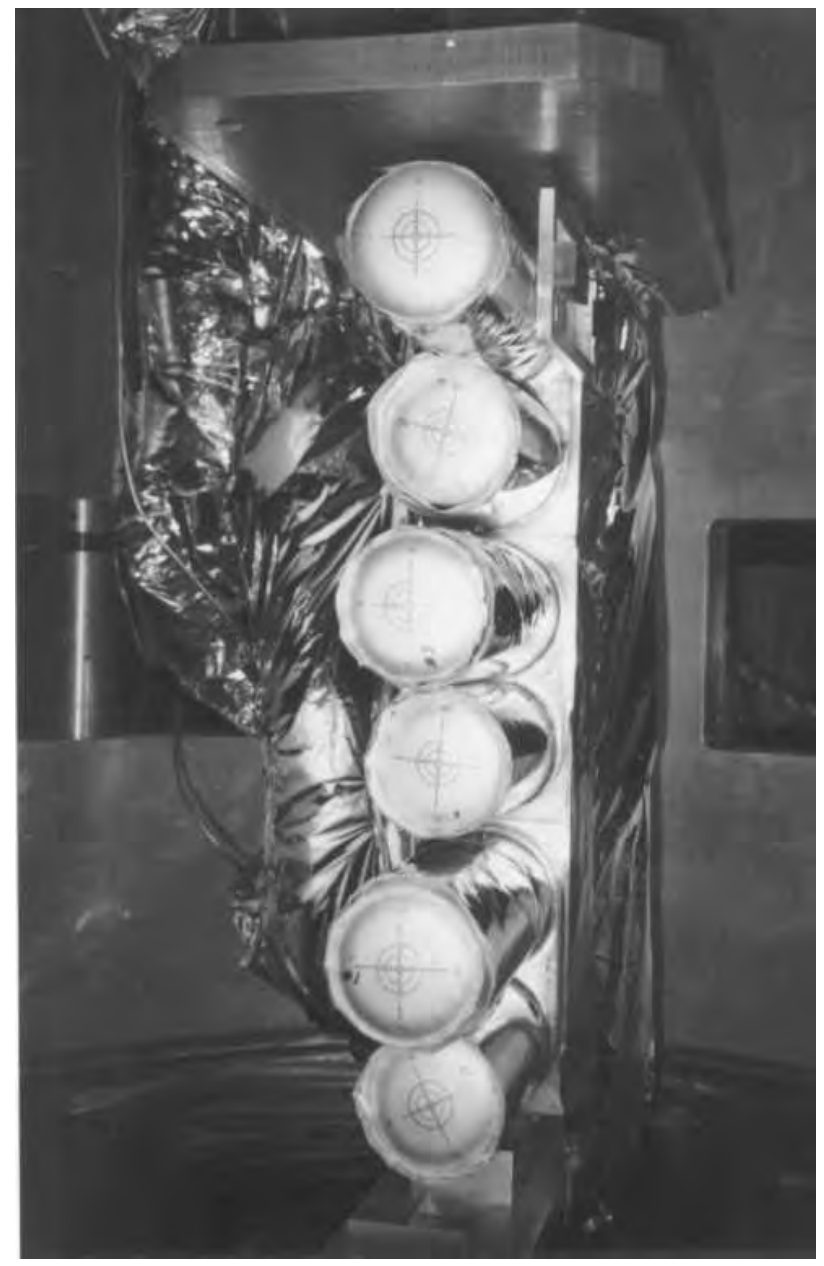

FIG. 13: Downstream view of the three cryotarget loops inside the scattering chamber. Some of the insulated target plumbing can be seen on the left. 
target. To maximize the magnetic structure function's contribution to the cross section, the electron spectrometer was placed at its extreme backward scattering angle $\left(144.5^{\circ}\right)$. At this scattering angle, a portion of the scattered electrons would re-scatter from the target cell block (the metal block to which the targets are attached, see Figures 14 and 15). In order to reduce this effect's contribution, a collimator was installed on the $15 \mathrm{~cm}$ target cell of Loop 1. The use of this collimator was unnecessary during the $A\left(Q^{2}\right)$ data taking (during which the scattered electrons were detected at forward angles), so to minimize mechanical changes to the target, liquid deuterium was switched to Loop 3 (which had no collimator) and the cryogen in Loop 1 was changed to gaseous ${ }^{4} \mathrm{He}$. The target remained in this configuration for the rest of the data taking. Note that the gaseous ${ }^{4} \mathrm{He}$ loop is not used as a target. It functions as a spare loop which will be utilized in the future. The whole target ladder shown in Figure 12 is connected to a vertical lifting mechanism so that the desired target cell can be placed in the beam's path. In the target ladder's uppermost vertical position, the beam passes through the empty target, straight to the beam dump.

A diagram of one of the loops can be seen in Figure 14. The main components in each loop are the heat exchanger, the axial fan, the cell block, the heaters and the temperature thermometry.

The heat exchanger is seen at the top of Figure 14. Inside the heat exchanger are three concentric cylinders filled with fin-tubing. Gaseous ${ }^{4} \mathrm{He}$ from the End Station Refrigerator provides cooling for the target liquid. It flows through the tubing in the heat exchanger at a nominal rate of 10 grams/s (the flow can be adjusted depending upon the amount of cooling power needed) with a temperature of $15 \mathrm{~K}$ and a pressure of 11 atmospheres. The axial fan, located at the center of the heat exchanger, pumps the target cryogen around the fin-tubing, in the direction denoted by the arrows, from the heat exchanger to the cell block and back again.

A side view of a cell block and its pair of target cells can be seen in Figure 15. The target cells themselves are thin cylinders made from Aluminum beer can stock. The cans have a diameter of $6.48 \mathrm{~cm}$ and a sidewall thickness of $0.18 \mathrm{~mm}$ 


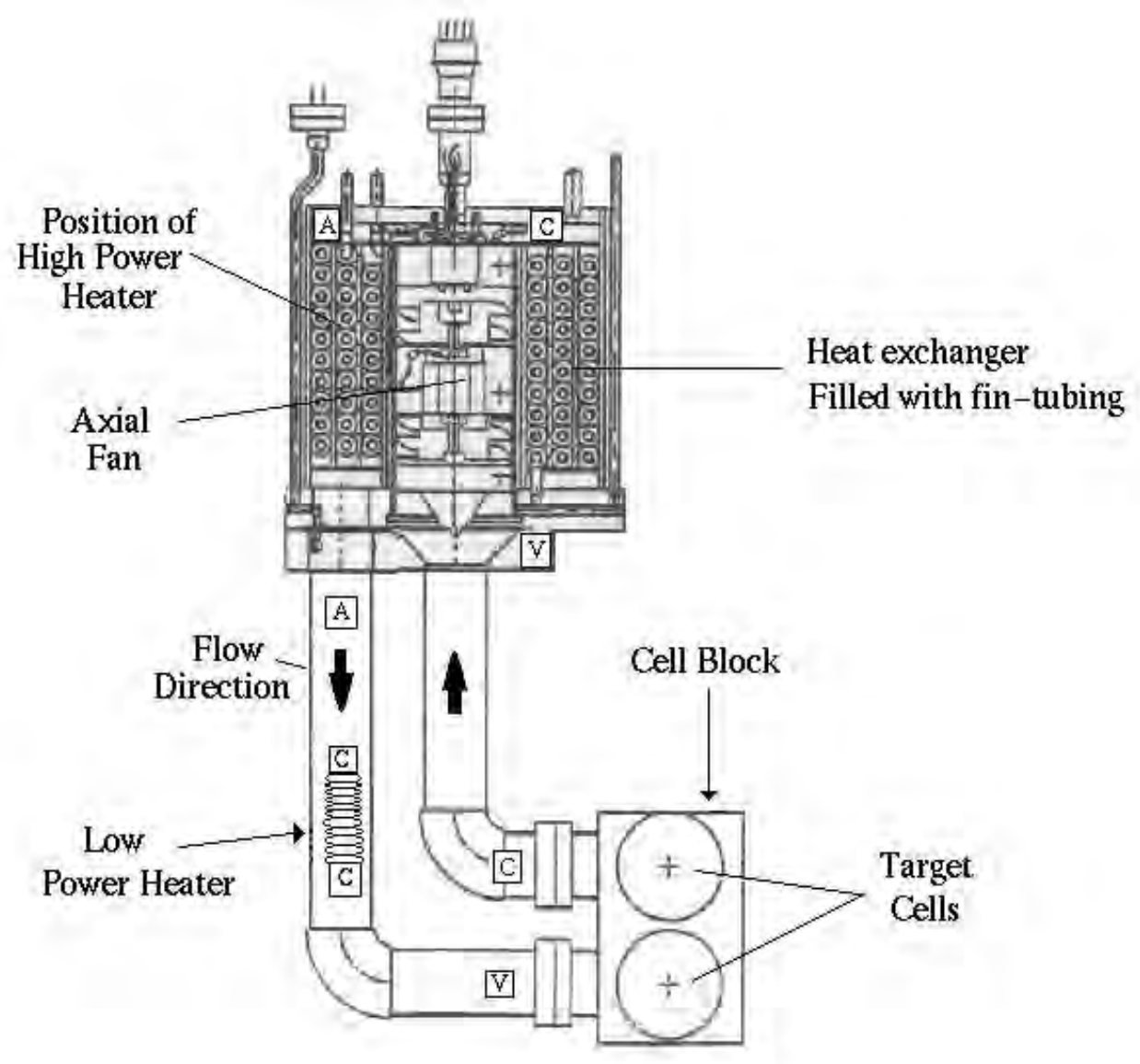

FIG. 14: Diagram of a single target loop. Shown are all of the main loop components. The squares represent the three types of temperature sensors: (C)ernox, (A)llen-Bradley and (V)apor pressure bulbs. 


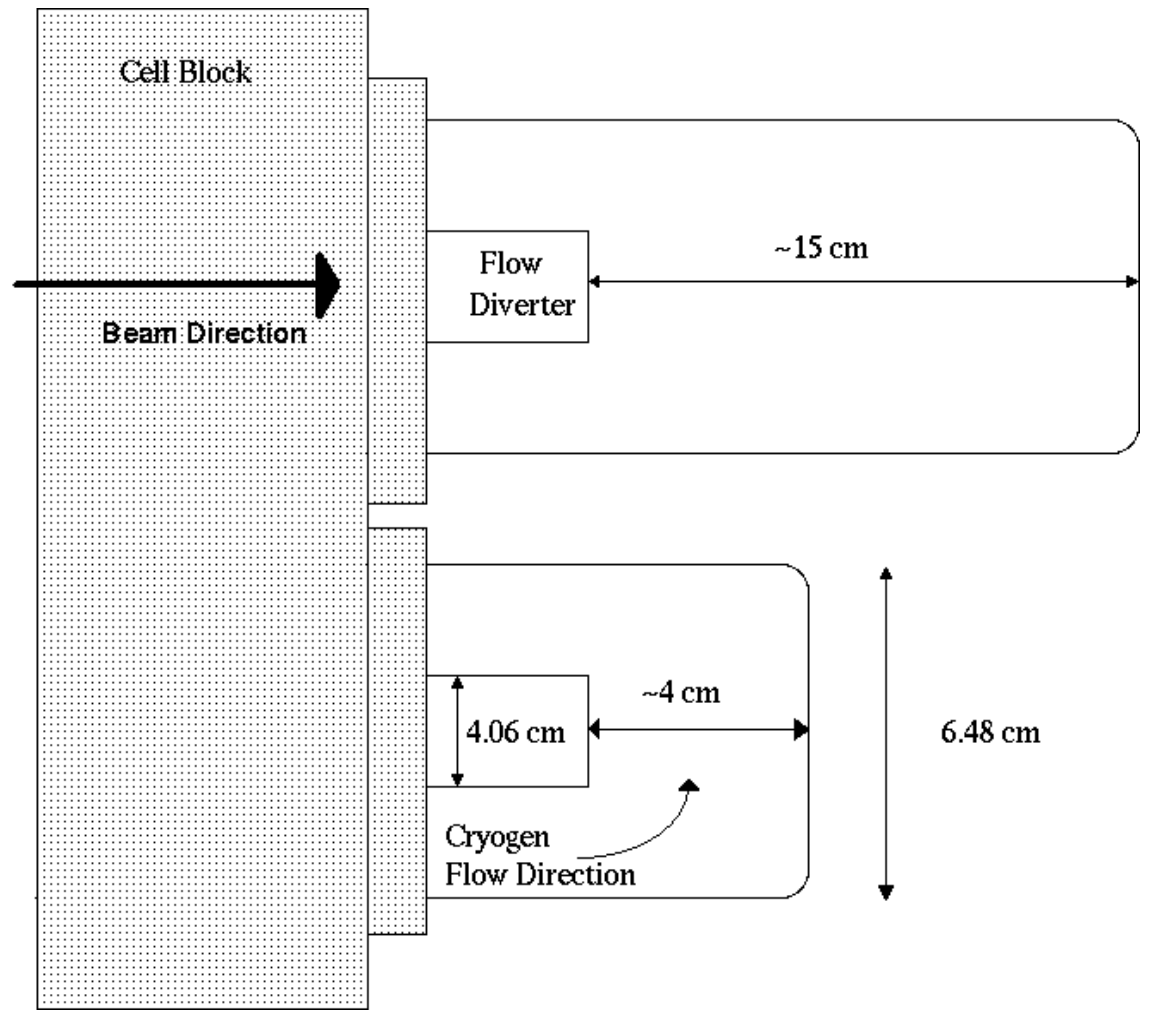

FIG. 15: Side view of a target cell block. Shown are the cell block's pair of target cells and their dimensions. 
TABLE I: Cryotarget dimensions (cm).

\begin{tabular}{|c|c||c|c|c|}
\hline Target Loop & Cell & Cold Length & $\begin{array}{c}\text { Upstream } \\
\text { Window } \\
\text { Thickness }\end{array}$ & $\begin{array}{c}\text { Downstream } \\
\text { Window } \\
\text { Thickness }\end{array}$ \\
\hline \hline 1 & $15 \mathrm{~cm}$ & $14.91 \pm 0.02$ & $0.0071 \pm 0.0003$ & $0.0107 \pm 0.0005$ \\
& $4 \mathrm{~cm}$ & $3.91 \pm 0.01$ & $0.0071 \pm 0.0003$ & $0.0117 \pm 0.0005$ \\
\hline 2 & $15 \mathrm{~cm}$ & $14.95 \pm 0.02$ & $0.0071 \pm 0.0003$ & $0.0094 \pm 0.0005$ \\
& $4 \mathrm{~cm}$ & $3.78 \pm 0.01$ & $0.0071 \pm 0.0003$ & $0.0089 \pm 0.0005$ \\
\hline 3 & $15 \mathrm{~cm}$ & $14.94 \pm 0.02$ & $0.0071 \pm 0.0003$ & $0.0097 \pm 0.0005$ \\
& $4 \mathrm{~cm}$ & $3.93 \pm 0.01$ & $0.0071 \pm 0.0003$ & $0.0091 \pm 0.0005$ \\
\hline
\end{tabular}

(see Reference [18] for all target measurements). Soldered onto the downstream end of the target cell can is an Aluminum endcap. The beam passes through this window after interacting with the target cryogen. The other end of the target can is soldered onto the cellblock. Inside each target cell is a flow diverter. This diverter forces the cryogen into the beam path. The beam passes through an upstream window at the end of the flow diverter before reaching the target cryogen. The target liquid flows through the two target cans from bottom to top, i.e. the cryogen flows first through the $4 \mathrm{~cm}$ cell and then through the $15 \mathrm{~cm}$ and back to the heat exchanger. The flow speed of the cryogen depends upon the rotation frequency of the axial fan that circulates the target fluid. This circulation rate was optimized during the target commissioning. The nominal target lengths are $15 \mathrm{~cm}$ and $4 \mathrm{~cm}$. The actual target lengths vary with construction and operating temperature. Table I lists the lengths of each target cell, corrected for their operating pressure (22 psia for Loop 1 and Loop 3 and 26 psia for Loop 2) and for thermal contraction (approximately $0.4 \%$ at $20 \mathrm{~K}$ ).

Two types of heaters were employed, knicknamed "high" and "low" power heaters. The high power heaters are kapton encased wires embedded in the heat exchanger. These heaters can provide more than 700 Watts of heat. They are used to regulate the temperature of the cryogen during periods when the electron 
beam is off. The low power heaters are located on the inlet side of the cellblock. They can provide up to $50 \mathrm{~W}$ of heating power and were used to compensate for small temperature variations caused by, for example, a small change in beam current.

The loop is monitored by several different types of thermometry, each with a particular range of temperatures for which it is calibrated. The temperature sensors which are most accurate at our cryogenic temperatures are known as Cernox Temperature Sensors. There are four of them per loop; two on the cryogen inlet side, one on the outlet side and one in the heat exchanger. These sensors provide resistive temperature measurements. The resistors are calibrated by the manufacturer (LakeShore) and the calibrations are loaded into a temperature monitoring unit which converts the measured resistance to temperature. There are two Allen-Bradley temperature sensors located in each loop, one on the cryogen inlet side and one in the heat exchanger. These sensors are less accurate at our cryogenic temperatures than the Cernox sensors and are used mostly as a visual check that the temperature is in the correct range. In addition, several of the Allen-Bradley temperature sensors are calibrated to $300 \mathrm{~K}$, so they are used to monitor the target's temperature during warm-up and cool-down periods where the target temperature is changed from approximately $20 \mathrm{~K}$ to $300 \mathrm{~K}$ and viceversa. The third type of temperature measurement is deduced from the vapor pressure inside a hydrogen filled bulb. There are two vapor pressure bulbs located in the target loop, with one on the cryogen inlet side and one in the heat exchanger. As with the Allen-Bradley sensors, this method is used as a visual check that the cryogen's temperature is in the correct range.

\section{Software}

The control system development environment known as EPICS (Experimental Physics and Industrial Control System) was used to develop the interfaces needed for remote control of the cryotarget hardware. The EPICS development environment consists of a collection of $\mathrm{C}$ codes and MOTIF programs, originally developed by Los Alamos National Laboratory and Argonne National Laboratory, but 
now in use in more than 90 independent projects, including particle and nuclear physics, astronomy and industrial applications [19].

The physical architecture of a control system developed in the EPICS environment is shown in the left half of Figure 16. A workstation (here an HP 745) runs the graphical user interfaces (GUIs) or other programs which communicate via ethernet to an IOC (Input/Output Controller). The IOC for the cryotarget is a Motorola MV162-532 chip on a circuit board located in a VME crate. The communication with the hardware is through the IOC. In the right half of Figure 16 is a schematic of the actual software tools which make the hardware communication possible. For the cryotarget, GUI development programs such as MEDM and TCL were used to develop control screens for each piece of hardware to be controlled. When the status of a hardware device is queried or changed, the GUI communicates to the IOC via a network protocol known as channel access. Explicitly, the GUI communicates with a database which runs on the IOC. This database contains multiple records for each device that is to be controlled. Most of these records refer to a specific function a device is to perform, such as reading the output of a temperature sensor or setting the position of a valve. The database is monitored by the record support. When a change or update of a hardware device is requested, the record support calls the device support routines necessary to query the hardware. Depending upon the complexity of the hardware, the device support may communicate directly with it or it may call a specialized driver support to do the communicating. This cycle continues over and over, for each change or update of a device's status.

The control system for the Hall A target was produced entirely in the EPICS environment. There are 13 serial devices, two relay boards and five I/O boards that are queried and controlled, on time scales of 0.1 to 10 seconds. The development of the Hall A control system and its associated GUIs built upon the work done previously for the Hall C cryogenic target. The changes and additions necessary for Hall A were mostly done by myself, Fraser Duncan (University of Maryland) and Johnny Tang (Accelerator Division) over a period of approximately nine months. The GUI contains approximately 40 subscreens which allow 


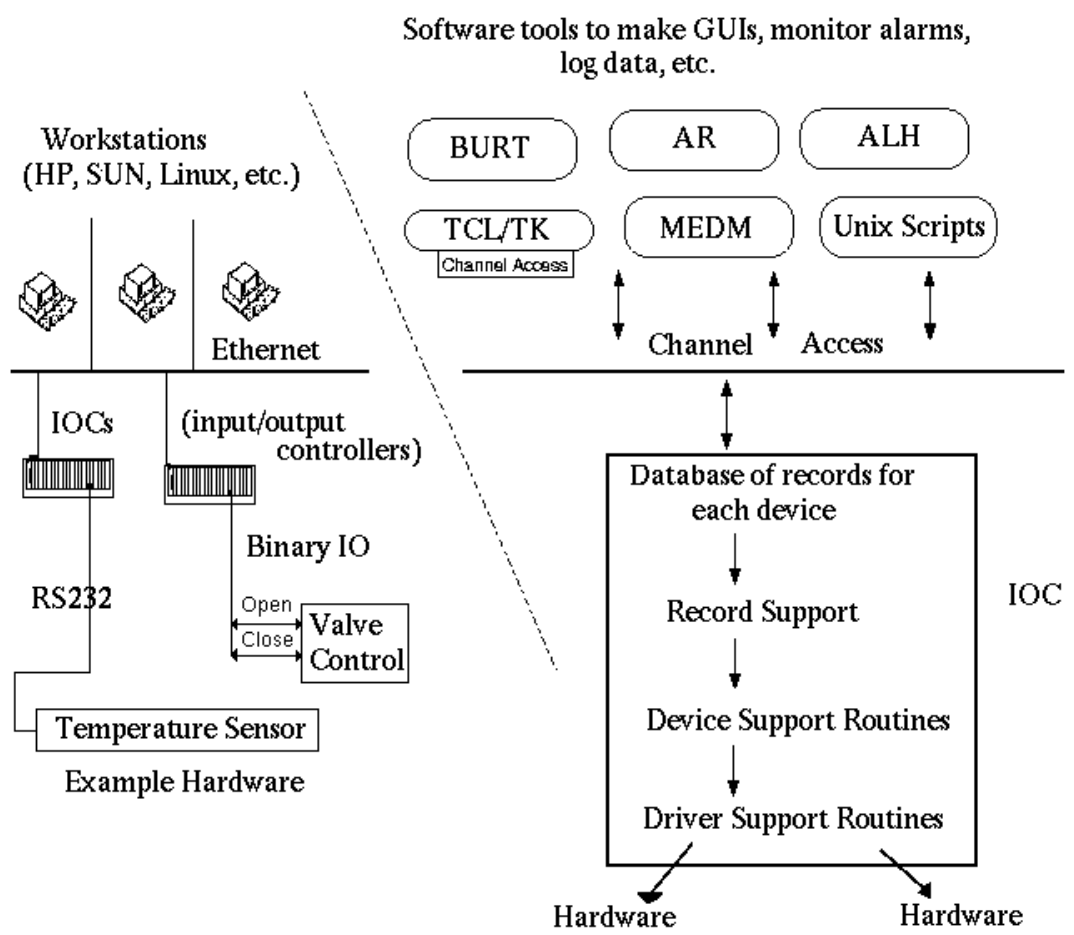

FIG. 16: Physical (left) and logical (right) architecture of a control system developed in the EPICS environment. 
TABLE II: Cryotarget parameters.

\begin{tabular}{|c||c|c|c|}
\hline Target Liquid & Temperature $(\mathrm{K})$ & Pressure $(\mathrm{psia})$ & Density $\left(\mathrm{g} / \mathrm{cm}^{3}\right)$ \\
\hline \hline Hydrogen & 19.00 & 26.0 & $0.07230 \pm 0.00007$ \\
\hline Deuterium & 22.00 & 22.0 & $0.1670 \pm 0.0005$ \\
\hline
\end{tabular}

communication with the 1200 records in the IOC's database.

In addition to the cryotarget slow controls, I have worked on several other hardware control projects utilizing the EPICS environment. The first such project involved working with a group from Jefferson Lab Hall B to develop a control system for the LeCroy 1458 high voltage mainframe. Each high voltage mainframe has the capability to output voltage on 192 independent channels. There are five of these high voltage mainframes in use in Hall A, providing high voltage to various detectors and photomultiplier tubes used in the spectrometer detector stacks and the beamline devices. The control system developed is in use in Hall A and a variation of it is used in Hall B. Recently, I have worked on debugging and upgrading the controls for the Hall A beam current monitoring system and the Hall A polarized ${ }^{3} \mathrm{He}$ target.

\section{Operating parameters}

The operating temperature and pressure of the hydrogen and deuterium target loops is shown in Table II. The temperature is determined by the Cernox resistive temperature measurements, while the pressure is measured by two pressure transducers, one located in the target fill line and the other in the target return line. The error on the density results from four sources. The first is the error on the measured temperature and pressure. The Cernox temperature measurements have an absolute accuracy of approximately $50 \mathrm{mK}$. The density dependence on temperature is $\frac{1}{\rho} \frac{d \rho}{d T}=-1.25 \% / \mathrm{K}$, which leads to an error of less than $0.1 \%$ in the density from temperature uncertainty. The pressure measurement from the pressure transducers has an error of about approximately 0.3 psia. The dependence 
of density on pressure is $\frac{1}{\rho} \frac{d \rho}{d P}=0.01 \% /$ psia, which leads to an error of much less than $0.1 \%$ in the density from the pressure measurement. The additional sources of error in the density are the uncertainty in the equations of state and uncertainty in our knowledge of the relative amounts of ortho vs. para molecules in the hydrogen and deuterium target fluid (because the two molecular states have different densities). These two sources combined contribute about $0.1 \%$ error in the density determination for hydrogen and about $0.3 \%$ error in the density determination for deuterium. All of these factors combined lead to an operating density of $0.07230 \pm 0.00007 \mathrm{~g} / \mathrm{cm}^{3}$ for hydrogen and $0.1670 \pm 0.0005 \mathrm{~g} / \mathrm{cm}^{3}$ for deuterium in the absence of beam.

With beam, there is an additional current-dependent uncertainty in the target density due to local boiling. Several efforts were made to study the target density variation as a function of beam current [20]. For all of these tests, the beam spot size (raster size) was maintained at $\pm 1.7 \mathrm{~mm}$ by $\pm 1.4 \mathrm{~mm}$ ( $x$ and $y$ dimensions, respectively). Figure 17 shows the results of the two boiling tests conducted on liquid hydrogen. Plotted is the analyzed yield (number of events, normalized to charge and deadtime) versus the incident beam current. Both data sets have been normalized to one at their lowest current points. The error bars are statistical and are approximately 1-2\%. The data sets agree within error bars in their region of overlap. Hydrogen shows a significant boiling effect above about $40 \mu \mathrm{A}$. This does not significantly affect our $\mathrm{H}\left(e, e^{\prime} p\right)$ calibration data because these data were taken at a beam current of $10 \mu \mathrm{A}$, where boiling effects are very small. Figure 18 shows the results of the two boiling tests conducted on liquid deuterium. The error bars are statistical and are approximately $0.5-3 \%$. The results of the deuterium boiling studies at high current are of importance because the majority of the data for the structure function measurement were taken at beam currents greater than $50 \mu \mathrm{A}$. Based on these boiling studies, a current-dependent density correction was applied for both hydrogen and deuterium data runs. This correction is described in Section 3.9.4. 


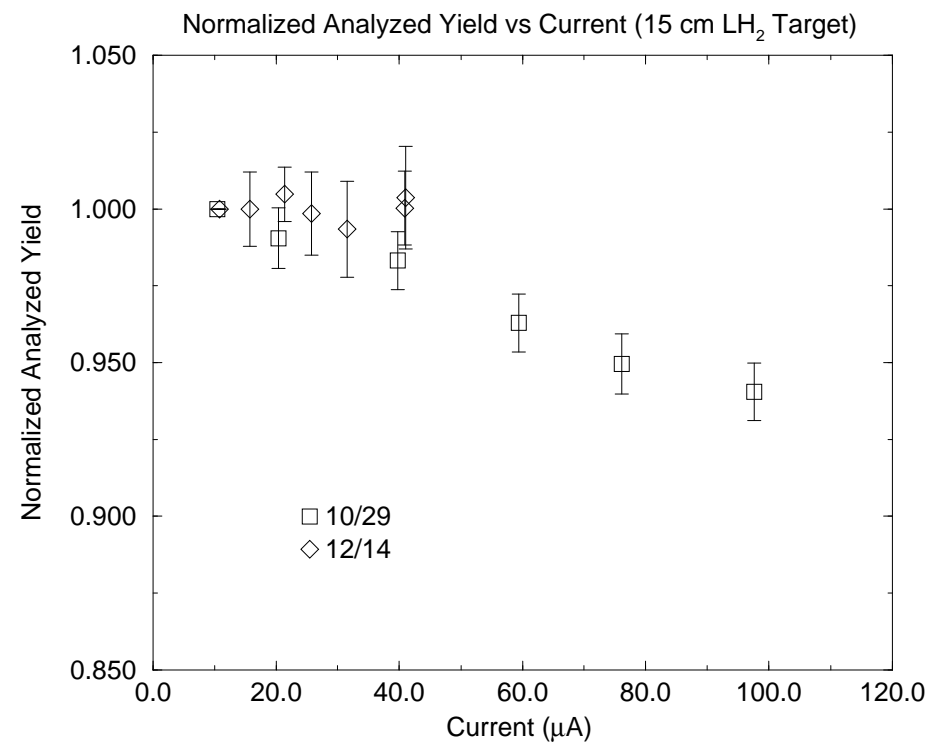

FIG. 17: Normalized yield versus current for the $15 \mathrm{~cm} \mathrm{LH2} \mathrm{target.} \mathrm{Shown} \mathrm{is}$ data taken on 10/29/97 and 12/14/97.

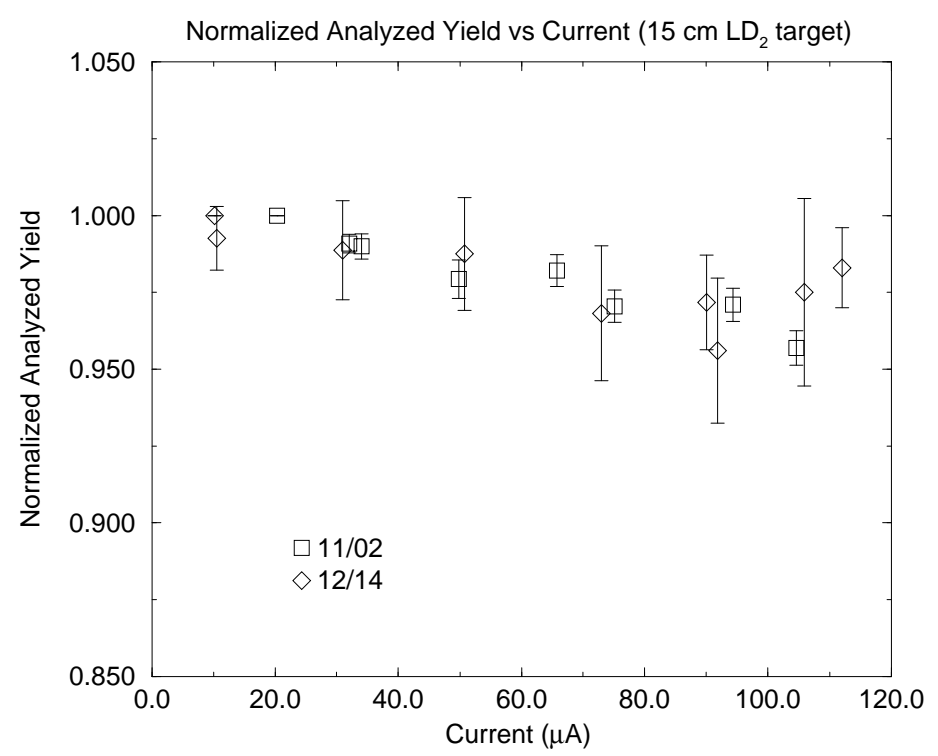

FIG. 18: Normalized yield versus current for the $15 \mathrm{~cm}$ LD2 target. Shown is data taken on 11/02/97 and 12/14/97. 
TABLE III: Solid target thicknesses.

\begin{tabular}{|c|c|c|}
\hline Target & Thickness $(\mathrm{mm})$ & Density $\left(\mathrm{mg} / \mathrm{cm}^{3}\right)$ \\
\hline $\mathrm{Al}$ & $\mathrm{N} / \mathrm{A}$ & $\mathrm{N} / \mathrm{A}$ \\
\hline $\mathrm{C}$ & $1.016 \pm 0.003$ & $223.20 \pm 0.14$ \\
\hline $\mathrm{Al}$ & $1.016 \pm 0.013$ & 2.71 \\
\hline $\mathrm{BeO}$ & $0.508 \pm 0.013$ & $\mathrm{~N} / \mathrm{A}$ \\
\hline Empty & $\mathrm{N} / \mathrm{A}$ & $\mathrm{N} / \mathrm{A}$ \\
\hline
\end{tabular}

\subsubsection{Solid Targets}

Attached to the bottom of the cryotarget ladder was a solid target ladder with three aluminum dummy targets and five solid target positions (see Figure 12). Each dummy target consists of two flat plates of aluminum, separated by empty space. The three dummy targets, with lengths of $10 \mathrm{~cm}, 15 \mathrm{~cm}$ and $4 \mathrm{~cm}$, were used to estimate the contribution of the target aluminum endcaps to the background. Below the dummy targets were the solid foil targets. On this ladder were two aluminum targets, one carbon target, one Beryllium-Oxide (BeO) target and one empty target with no target foil. Each target (except the empty target) was approximately $2.54 \mathrm{~cm}$ high and $1.91 \mathrm{~cm}$ wide. Table III lists the thicknesses of each target.

At the top of the solid target ladder is an aluminum target with two small holes (1 $\mathrm{mm}$ and $2 \mathrm{~mm}$ square) in it. This target was used during the fast raster commissioning, with the idea that the dimensions of the rectangular holes were known so the dimensions of a rastered beam passing through the holes could be discerned. Next are carbon and aluminum target foils. These targets can be used for spectrometer studies. Fourth on the solid target ladder is the Beryllium-Oxide $(\mathrm{BeO})$ target. When beam is incident on a BeO target, it causes the target to glow brilliantly. This target is used for a visual check that beam is present and in the correct position with a well defined shape. At the bottom of the solid target ladder is the empty target, which is essentially an aluminum foil with a circular 
hole cut in it. The empty target functions as the "target-out-of-beam" position, because when it is positioned in the beam's path, the beam goes through the hole, straight to the beam dump.

\subsection{High Resolution Spectrometers}

There are two superconducting high resolution spectrometers in Hall A which are nominally identical in terms of their magnetic properties. The spectrometer magnets are in a QQDQ (quad, quad, dipole, quad) configuration, as shown in Figure 19. The optical length of the spectrometer is 23.4 meters, with a $45^{\circ}$ vertical bending angle at the dipole. The spectrometers have a momentum range from 0.3 to $4.0 \mathrm{GeV} / \mathrm{c}$ and a momentum resolution of $10^{-4}$ (FWHM).

The entrance window to each spectrometer is separated from the scattering chamber exit windows by $20 \mathrm{~cm}$ of air. The entrance window is covered with 0.18 $\mathrm{mm}$ of Kapton foil. A box containing three tungsten collimators [21] is located between this entrance window and the first quadrupole (Q1). The upper collimator is a sieve slit (Figure 20). This collimator is $5 \mathrm{~mm}$ thick and has a $7 \times 7$ grid of holes bored in it; two of the holes are $4 \mathrm{~mm}$ in diameter and the rest are $2 \mathrm{~mm}$ in diameter. The sieve slit collimator is used during spectrometer optics studies. The middle collimator is an $8 \mathrm{~cm}$ thick $6 \mathrm{msr}(6.29 \mathrm{~cm}$ wide $\times 12.18 \mathrm{~cm}$ high at a distance of $110.9 \mathrm{~cm}$ from the target) rectangular collimator. This collimator is used during normal data taking so as to ensure that the limits of acceptance of the spectrometer are well defined. The bottom collimator is an "empty collimator" which performs no collimation. The empty collimator position was used during the high momentum transfer measurements so as to maximize the event rate. At the top of each spectrometer is a $0.10 \mathrm{~mm}$ thick Titanium exit window, situated between the spectrometer's last quadrupole (Q3) and the detector shield house.

The particles that traverse the spectrometer are detected in a detector package located in a shield house at the top of each spectrometer. The position and angle of incidence of the detected particles are measured at the spectrometer "focal plane" with a pair of vertical drift chambers. These measured coordinates can 


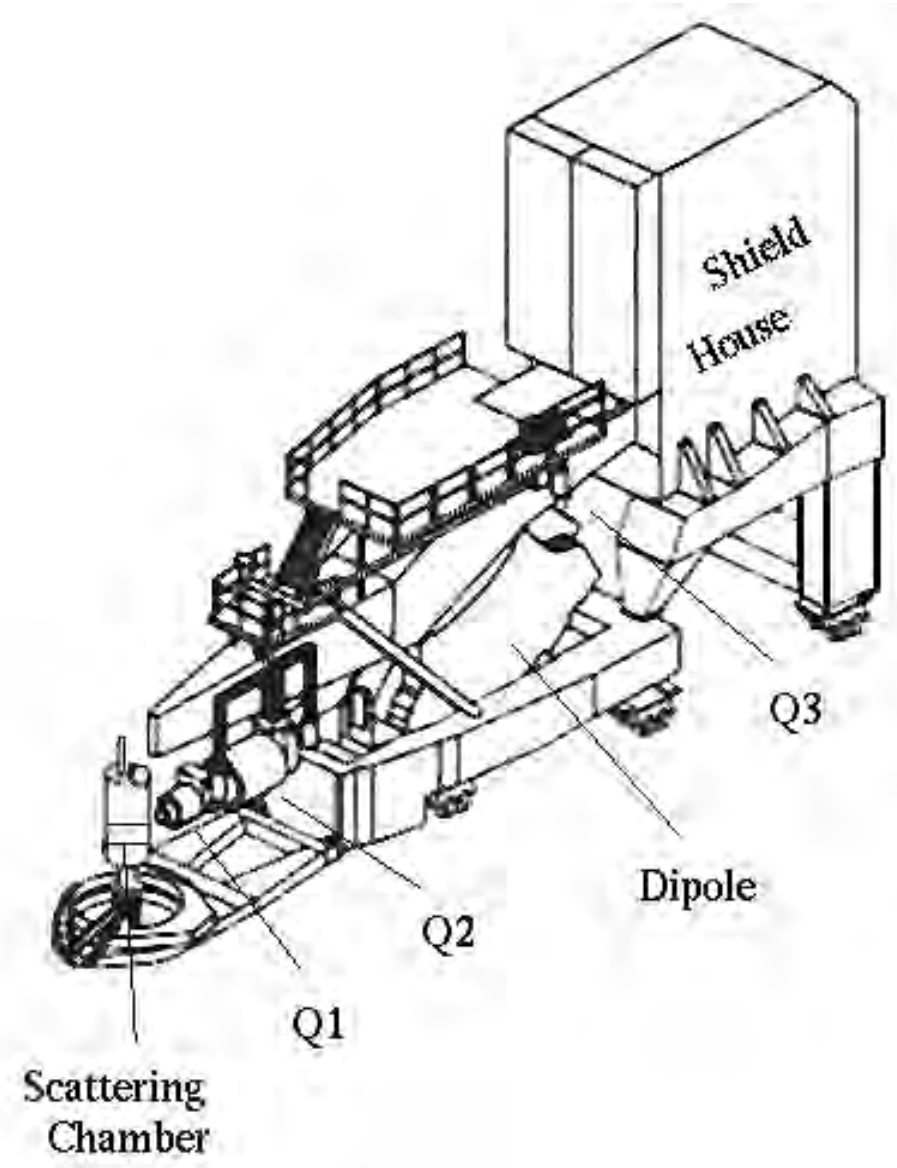

FIG. 19: Side view of a Hall A High Resolution Spectrometer. Shown are the scattering chamber and the spectrometer magnetic elements, including the dipole and the three quadrupole magnets (labeled Q1, Q2 and Q3). During data taking, the detectors sit inside the shield house at the top of the spectrometer. 


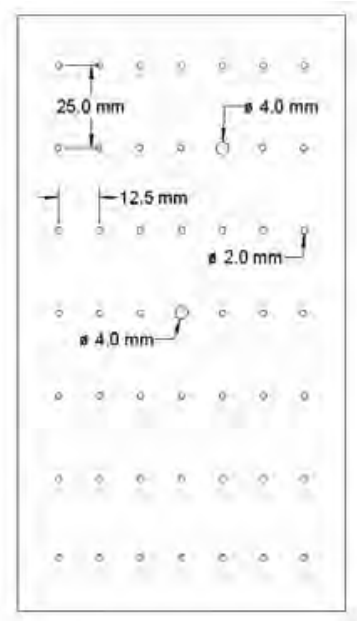

FIG. 20: Diagram of the sieve slit collimator used on the Electron Arm. The sieve slit collimator installed on the Hadron Arm is identical to that of the Electron Arm. It is installed flipped along the horizontal centerline relative to the HRSE sieve slit, so that its off-center $4 \mathrm{~mm}$ hole is in the bottom-right quadrant of the collimator instead of the top-right.

then be used to reconstruct the interaction point within the target via a set of optical traceback elements (see Section 3.3).

\subsection{Detector Packages}

The detector package for each spectrometer is mounted in a steel space frame. During data taking, the space frame and detectors sit inside a metal and concrete shield house where they are shielded from background radiation that could cause spurious events. The detector packages for the two spectrometers are shown in Figures 21 and 22. They are similar, each having two Vertical Drift Chambers (VDCs) and two planes of scintillators (S1 and S2). For this experiment, these were the only detectors employed on the hadron spectrometer. Additionally, the electron spectrometer's detector package contained a $\mathrm{CO}_{2}$ gas Čerenkov detector 


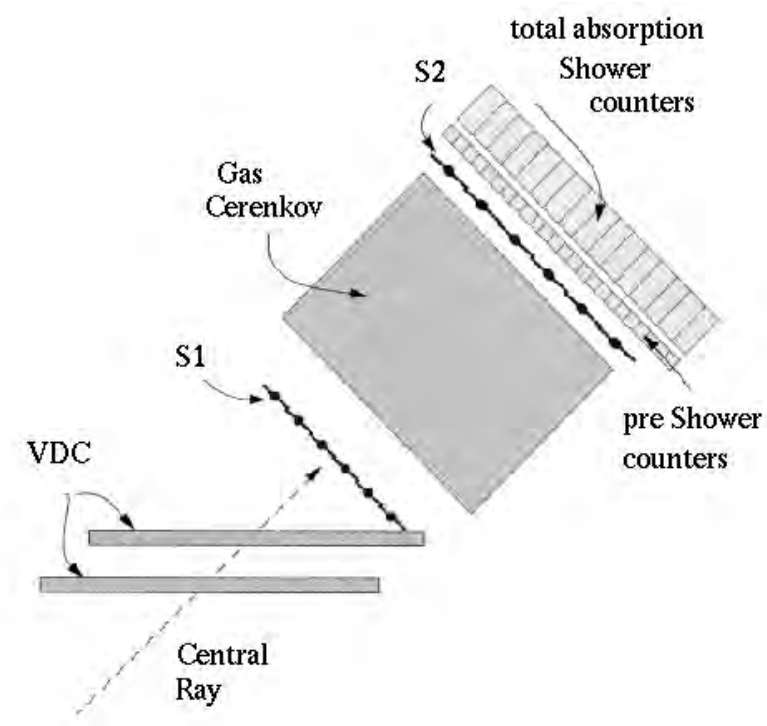

FIG. 21: Schematic of the Electron Arm detector package as used during this experiment.

and two arrays of lead-glass blocks acting as a pre-shower and a shower detector, respectively. In the following sections, a brief description of each detector will be given. The detector efficiencies will be discussed in Chapter 3 .

\subsubsection{Vertical Drift Chambers}

In order to determine the position and angle of incidence of particles passing through the detector package, each spectrometer has two Vertical Drift Chambers (VDCs) separated by $50 \mathrm{~cm}$. For a complete discussion of the VDCs, see Reference [22]. The VDCs are mounted on permanent rails on the floor of the shield house, between the spectrometer Q3 exit window and the detector space frame. As shown in Figure 23, each VDC has two wire planes, perpendicular to each other, in a standard UV configuration. The active area of each VDC is $211.8 \mathrm{~cm} \times 28.8 \mathrm{~cm}$ in the dispersive and transverse directions, respectively. The position resolution 


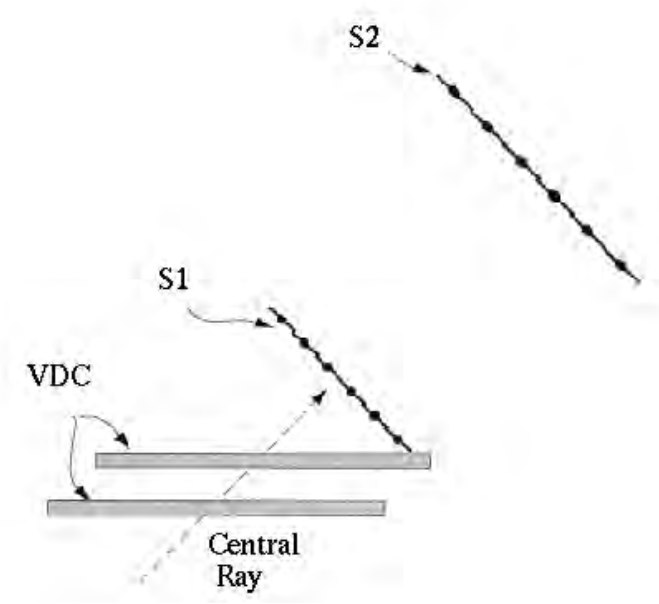

FIG. 22: Schematic of the Hadron Arm detector package as used during this experiment.

of each plane is approximately $225 \mu \mathrm{m}$ (FWHM). Each wire plane contains 368 signal wires. The signal wires are $20 \mu \mathrm{m}$ in diameter and are made of gold-plated Tungsten. Each wire plane is oriented at $45^{\circ}$ with respect to the spectrometer central ray.

Inside each VDC, there are three planes of negative high voltage $(-4000 \mathrm{~V}$ nominal), surrounding the two planes of grounded signal wires. The chamber gas is a mixture of Argon (Ar) and Ethane $\left(\mathrm{C}_{2} \mathrm{H}_{6}\right)$. When a charged particle passes through the VDC, it produces ions in the gas mixture. The electrons drift along the electric field lines defined by the high voltage planes and the signal wires. The large electric fields near the signal wires give rise to ionization avalanches resulting in detectable signals. These signals are then amplified and discriminated. The resulting logic pulses start multihit TDCs (time-to-digital converters), which are stopped by the event trigger. 

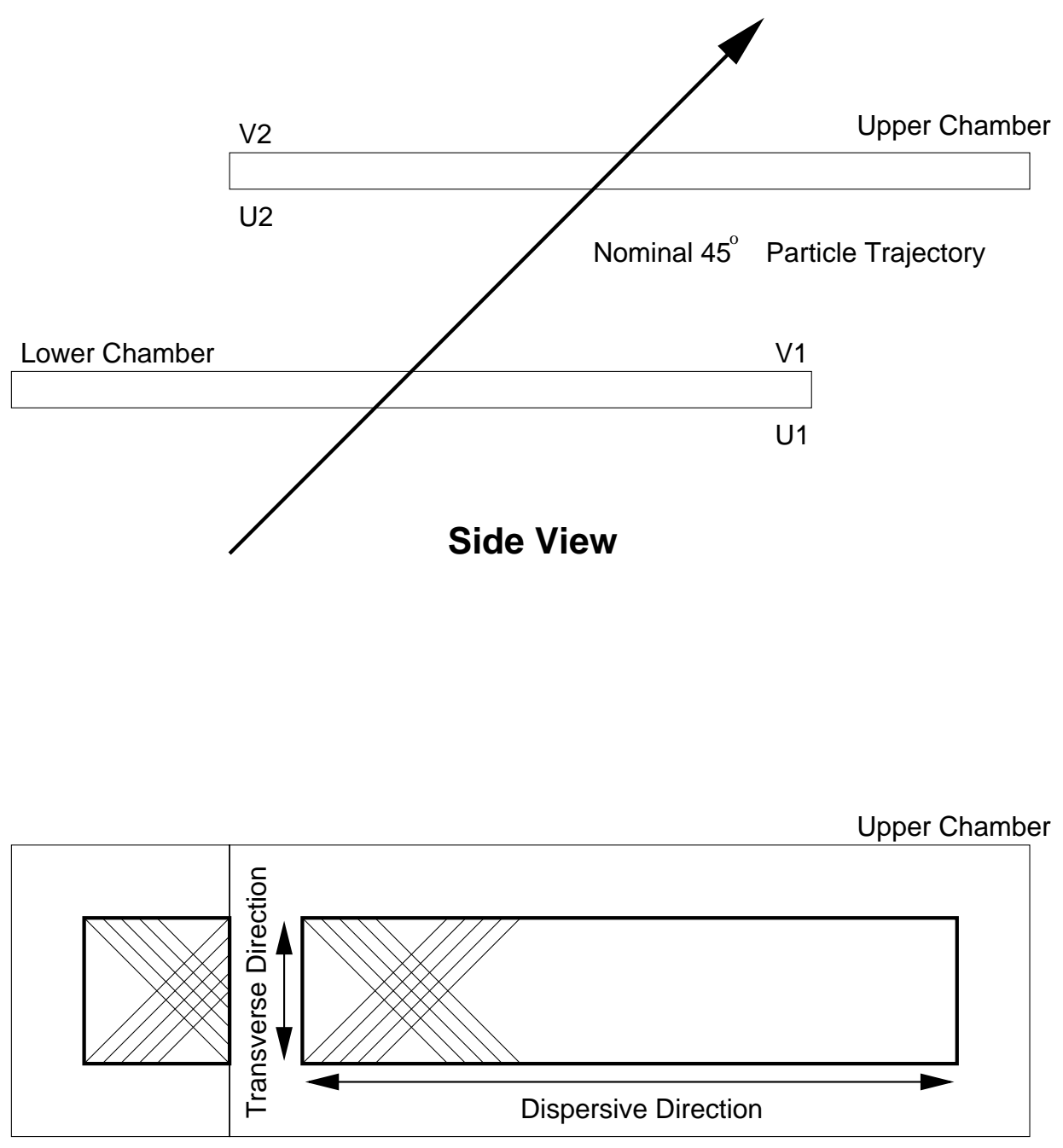

Lower Chamber

Top View

FIG. 23: Schematic of the two Vertical Drift Chambers (VDCs) of the Electron Spectrometer. The two in the Hadron Spectrometer are identically configured. 


\subsubsection{Scintillator Hodoscope}

Each detector package contains two planes of trigger scintillators, S1 and S2. The two planes are separated by 2 meters. Each plane of scintillators consists of six scintillator paddles, made of Bicron BC-408 plastic. Two Burle 8575 two inch photomultiplier tubes (PMTs) view each scintillator (one on each side). The active area of the $\mathrm{S} 1$ scintillator paddles is $36 \mathrm{~cm}$ (transverse) $\times 29.3 \mathrm{~cm}$ (dispersive) $\times$ $0.5 \mathrm{~cm}$. The paddles in plane S2 are slightly larger, with an active area of 60 $\mathrm{cm}$ (transverse) $\times 37 \mathrm{~cm}$ (dispersive) $\times 0.5 \mathrm{~cm}$. The scintillator paddles of each plane overlap by $5.0 \mathrm{~mm}$ in order to ensure complete coverage of the detector plane. As seen in Figures 21 and 22, the scintillators are oriented so that they are perpendicular to the spectrometer central ray. The scintillators are used, either alone or in combination with another detector (e.g. the gas Čerenkov), to provide the physics event triggers.

\subsection{3 Čerenkov Detector}

To discriminate between pions and electrons, a threshold gas Čerenkov detector was employed [23]. A Cerenkov detector operates on the principle that when a charged particle travels through the detector medium, it emits Čerenkov light if it travels faster than light would in that same medium (i.e. $v \geq \frac{c}{n}$, where $n$ is the index of refraction of the detector medium). The Cerenkov light is emitted in a cone about the particle's trajectory with an apex angle given by $\cos \theta=\frac{1}{n \beta}$.

The Cerenkov detector employed in the HRSE used 2780 liters of $\mathrm{CO}_{2}$ as the detector medium. The $\mathrm{CO}_{2}$ was at atmospheric pressure, leading to an index of refraction of $n=1.00041$. With this index of refraction, the minimum particle momentum for the production of Cerenkov light is $17 \mathrm{MeV} / \mathrm{c}$ for electrons and 4.8 MeV/c for pions. Note that the threshold momentum for pions is above the maximum momentum for the spectrometer, so pions could only give a Cerenkov signal through the production of knock-on electrons.

Mechanically, the detector was a rectangular tank. The dimensions of the sensitive area of the detector were $1.996 \mathrm{~m}$ in the dispersive $x$-direction, $0.558 \mathrm{~m}$ 

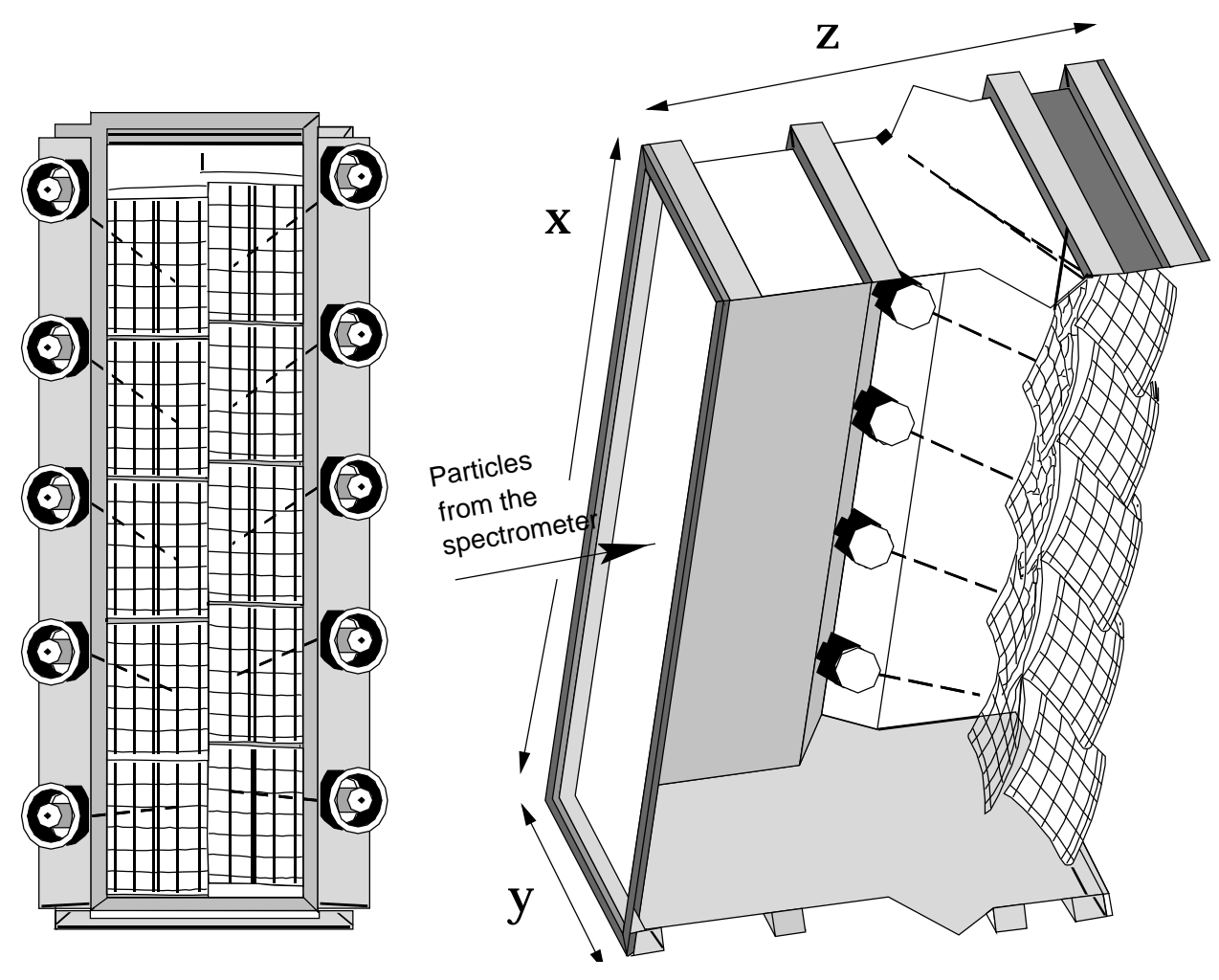

FIG. 24: Electron Arm $\mathrm{CO}_{2}$ gas Čerenkov detector. 
in the transverse $y$-direction and $1.5 \mathrm{~m}$ along the beam $z$-direction (see Figure 24). The entrance and exit windows of the detector were made of Tedlar foils (two $37.5 \mu \mathrm{m}$ thick films per window). The emitted Čerenkov light was reflected from ten aluminum mirrors placed just before the detector exit window. Each mirror had a spherical surface with radius of curvature of $90 \mathrm{~cm}$ and reflected the light to 5" photomultiplier tubes (PMTs) placed at the side of the detector box. The ten PMTs were of type Burle 8854, with a sensitive photocathode area of approximately $110 \mathrm{~mm}$ in diameter. The quantum efficiency of the tubes was $22.5 \%$ at $385 \mathrm{~nm}$, with a lower wavelength limit of $220 \mathrm{~nm}$. As a test, one of the ten PMT's of the detector was coated with p-Terphenyl $\left(\mathrm{C}_{18} \mathrm{H}_{14}\right)$. This coating absorbed UV light in the range from 110-360 $\mathrm{nm}$ and re-emitted it at about 385 $\mathrm{nm}$, increasing the photon yield in the sensitive range of the PMT.

\subsubsection{Pre-shower and Shower Detectors}

The last detectors in the electron arm are segmented lead glass shower counters. When charged particles are incident on the lead glass, they emit Bremsstrahlung radiation which can create secondary particles. In turn, some of these secondaries will interact, and so on, leading to a shower of particles. The number of photons produced by a shower is proportional to the charged track length, which is the sum of the distances traveled by the charged particles in the shower [24]. The charged track length is proportional to the energy of the incident particle, so monitoring the number of photons produced by a shower gives a measure of the incident particle energy.

The shower detector employed in the HRSE is divided into two sections. The first of the two sections is the 3.65 radiation length (r.l.) pre-shower detector, where a radiation length is defined as the average distance over which an electron's energy is reduced by a factor of $1 /$ e. The other is the 15.22 r.l. shower counter, which serves as a total absorption counter. The shower detectors are divided so as to make $e^{-}, \pi^{-}$discrimination easier. The electron has a high probability of starting a shower in the pre-shower detector relative to the pion, since it is lighter 
TABLE IV: Properties of the lead glass used as the pre-shower (TF-1) and shower (SF5) detectors.

\begin{tabular}{|c|c|c|}
\hline \hline Type & TF-1 Lead Glass & SF5 Lead Glass \\
\hline Radiation Length $(\mathrm{cm})$ & 2.74 & 2.38 \\
\hline $\begin{array}{c}\text { Total Radiation } \\
\text { Lengths of Detector }\end{array}$ & 3.65 & 15.22 \\
\hline
\end{tabular}

and therefore radiates more energy. By looking at the sum of the energy deposited in both the shower and pre-shower detectors versus the energy deposited in the pre-shower alone, electrons and pions can be distinguished.

Some of the physical properties of the lead glass used in the pre-shower and shower detectors are listed in Table IV. The pre-shower detector consists of 48 blocks of TF-1 lead glass, with dimensions $10 \mathrm{~cm} \times 10 \mathrm{~cm} \times 35 \mathrm{~cm}$ long. The blocks are arranged in an array of 24 blocks $\times 2$ columns, touching end to end. A Hammamatsu three inch R3036 PMT is affixed to one side of each block. The shower detector consists of 96 blocks of SF5 lead glass, in an array of 16 blocks $\times$ 6 columns. Unlike the pre-shower detector where the lead glass blocks lay flat on their support, the lead glass blocks for the shower counter stand on end, pointing outward along the direction of the central ray. Each block is $15 \mathrm{~cm} \times 15 \mathrm{~cm} \times 35$ cm tall. Each lead glass block is viewed by a 5" Philips XP2050 PMT.

\subsection{Trigger Types and Electronics}

For this experiment, the trigger electronics of both spectrometers were very similar. The singles triggers for both spectrometers (referred to as S1 and S3 below) were formed by the coincidence between its two scintillator planes, known as S1 and S2. The coincidence trigger was an AND of the triggers from the two single spectrometers. 
TABLE V: Trigger types.

\begin{tabular}{|c|c|c|}
\hline & Trigger Type & Description \\
\hline \multirow{5}{*}{$\begin{array}{c}\text { Raw } \\
\text { Triggers }\end{array}$} & S1 & HRSE S1 AND S2 (S-Ray) \\
\hline & $\mathrm{S} 2$ & $\begin{array}{c}\text { NOT S-Ray AND one missing of HRSE S1,S2, } \\
\text { Čerenkov }\end{array}$ \\
\hline & S3 & HRSH S1 AND S2 (S-Ray) \\
\hline & S4 & $\begin{array}{l}\text { NOT S-Ray AND One missing of } \\
\text { HRSH S1,S2 }\end{array}$ \\
\hline & $\mathrm{S} 5$ & S1 AND S3 in coincidence \\
\hline \multirow{5}{*}{$\begin{array}{c}\text { Accepted } \\
\text { Triggers }\end{array}$} & $\overline{\mathrm{TT} 1}$ & 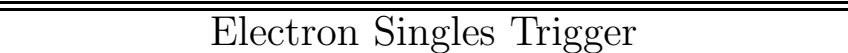 \\
\hline & $\mathrm{T} 2$ & Electron "Junk" Trigger \\
\hline & T3 & Hadron Singles Trigger \\
\hline & $\overline{\mathrm{T} 4}$ & Hadron "Junk" Trigger \\
\hline & $\mathrm{T} 5$ & Coincidence Trigger \\
\hline
\end{tabular}

A simplified diagram of the trigger electronics is seen in Figure 25. The full diagrams of the HRSE trigger circuit, the HRSH trigger circuit and the Coincidence circuit can be found in Appendix A.

The trigger types for this experiment are presented in Table V. An electron singles trigger, S1, was formed by a signal from the two HRSE scintillator planes $\mathrm{S} 1$ and S2 in coincidence. The signal had to satisfy the S-Ray requirement; that is, it had to have a hit-pattern such that if paddle $\mathrm{N}$ was hit on HRSE S1 then paddle $\mathrm{N}$ (or $\mathrm{N}+1$ or $\mathrm{N}-1$ ) had to be hit on HRSE S2. A hadron singles trigger, S3, was formed by a signal from the HRSH S1 and S2 in coincidence. It also had to have an S-Ray hit-pattern. Each spectrometer also had a "junk" trigger. The HRSE "junk" trigger, S2, had to have a non-S-Ray hit-pattern and allowed one of the S1, S2 or Čerenkov signals to be missing. The HRSH "junk" trigger, S4, had to have a non-S-Ray hit-pattern and allowed one of the S1 or S2 signals to be missing. These junk triggers were used for detector efficiency studies. The singles triggers, S1 and S3, are sent to an AND circuit to form the coincidence trigger, S5. 


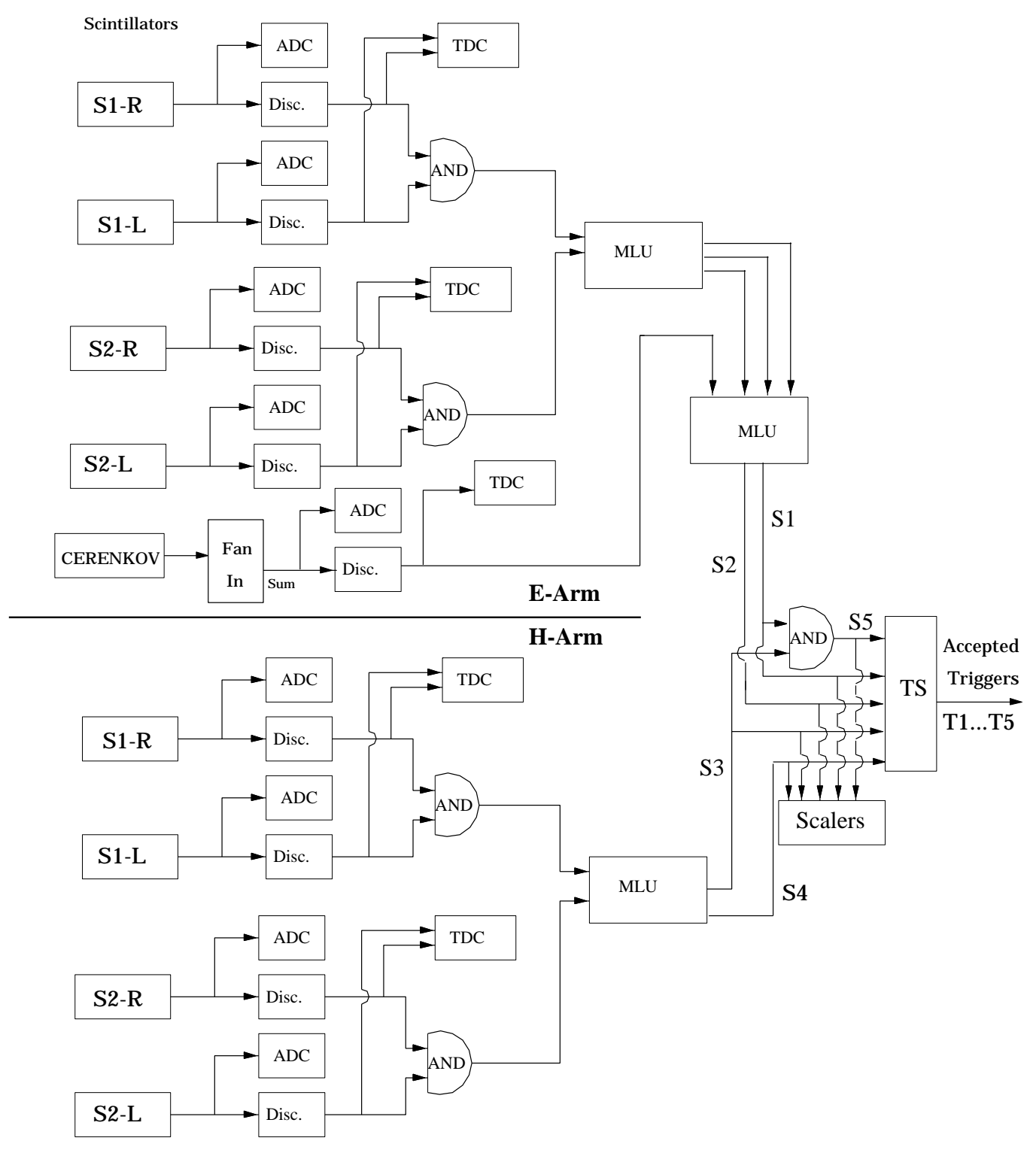

FIG. 25: A simplified diagram of the HRSE, HRSH and Coincidence trigger circuits used for this experiment. Note that none of the delays are shown. S1-S5 denote the raw trigger signals, while $\mathrm{T} 1-\mathrm{T} 5$ denote the accepted triggers. 
Each raw trigger is counted by the scalers and fed into the Trigger Supervisor (TS). The Trigger Supervisor first performs any requested prescaling of the raw triggers. The prescale factors are set by the experimenter and determine the fraction of events of a given type to be accepted by the TS. For example, if the prescale factor for a given trigger type is 5 , then the Trigger Supervisor ignores 4 events of that type and accepts the 5th. Then, if the TS isn't busy processing a previous event, it accepts the trigger. Once a trigger is accepted, the TS is dead for approximately $700 \mu$ s during which no other triggers are accepted while the event information is recorded (see Section 2.9).

\subsection{Data Acquisition}

The data acquired during E91-026 were a mixture of physics event data (acquired from the spectrometers for each accepted trigger), scaler data and data from the hardware slow controls systems (also known as EPICS events). All data were acquired using a data acquisition (DAQ) system built under the CODA (CEBAF On-line Data Acquisition) environment, version 1.4. For a detailed explanation of CODA and its capabilities, see Reference [25]. The data acquisition is controlled via a graphical user interface known as RunControl. Data acquired are written to a local computer disk and copied at specified times to the Mass Storage System (MSS) where it is archived on data tapes.

\subsubsection{Coda Overview}

CODA was developed at Jefferson Lab by the Data Acquisition Group. It is a toolkit from which application specific data acquisition systems can be developed, analogous to EPICS (which is a toolkit from which hardware slow controls systems can be developed). A schematic of the data acquisition system used in this experiment is shown in Figure 26. When particles pass through the detector package of either the electron or hadron spectrometers, they can generate signals in the scintillators and Čerenkov detector. If the pattern of detector signals is recognized 


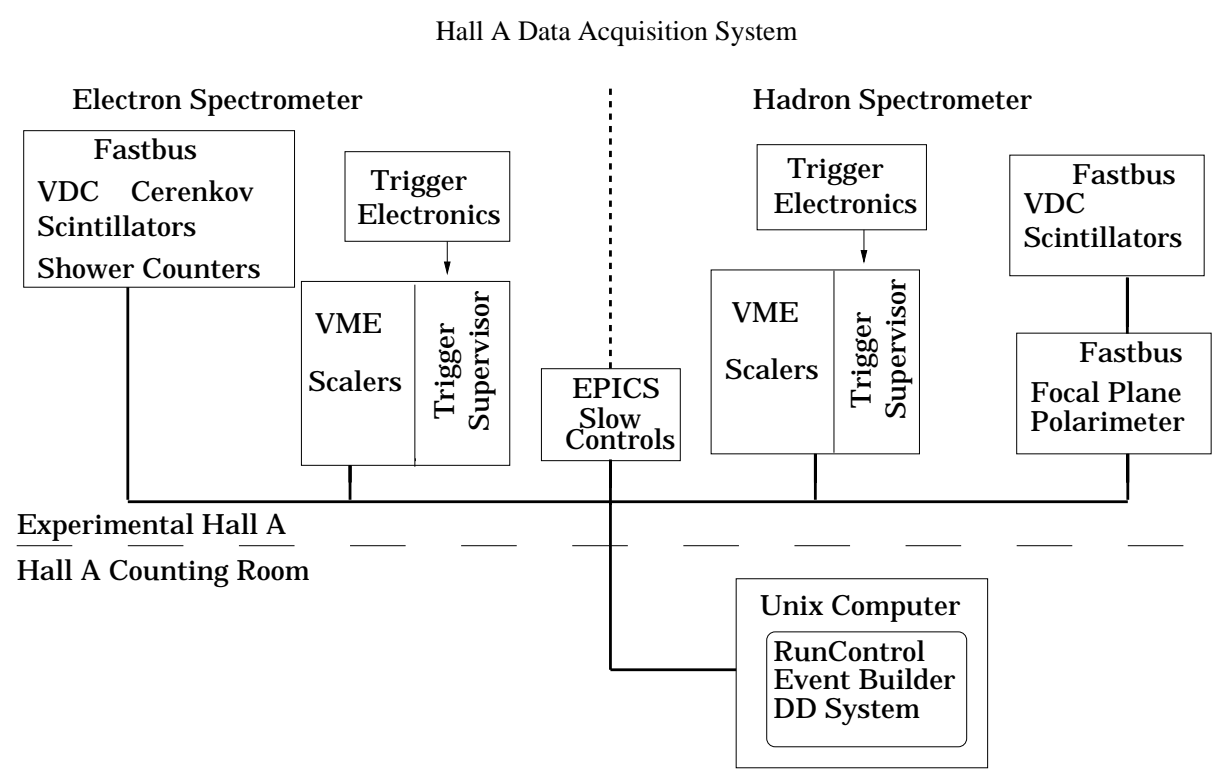

FIG. 26: Schematic of the Hall A DAQ.

by the trigger electronics as one of the allowed trigger types (see section 2.8 for allowed types), the Trigger Supervisor (TS) causes an event to be recorded by the data acquisition. First, the Read-Out Controllers (ROCs) are read out. There are four ROCs in the Hall A DAQ. The ROCs are single board computers in the FASTBUS and VME crates. These crates contain the ADCs, TDCs and scalers which contain the physics event information. The fragments of information from the ROCs are then collected by the Event Builder (EB) and put together into the CODA event format, including header and identifying information. After the event is built by the EB, it is written to disk for later analysis by the Hall A analysis program ESPACE, which is described in Section 3.2.

While data are being acquired, they can be examined using the Coda Data Distribution (DD) system. The DD system maintains a real-time event buffer which can be accessed by software tools. During this experiment, the program DHIST [26], which utilized the DD system to access the data in real-time, was 
used to make diagnostic histograms so that the quality of data could be monitored online.

\subsubsection{Coda Data File}

The data file generated by CODA is a mixture of status events, physics events and "special" events [27]. The status events are events such as PRESTART, GO, PAUSE and END that are included whenever the state of the run changes.

Spread throughout the data file are the physics event data. These data are arranged in an array of 32-bit words. The first part of the event structure contains "header" information, including, for instance, the event length, the event type (e.g. T1-T5) and the run number. The physics event data follow the header and contains information extracted from the four ROCs. Three of the ROCs provide the information from the detectors, while the forth ROC provides BPM and raster data which can later be used to reconstruct the interaction point of an event within the target. Typical coincidence event rates ranged from $200 \mathrm{~Hz}$ at low $Q^{2}$ to several $\mathrm{Hz}$ for the high $Q^{2}$ data points.

"Special events" are events such as scaler readouts and EPICS data from the detector and beamline elements not directly associated with the DAQ. The scalers count the raw hits on the detector phototubes, as well as the number of triggers generated by the trigger supervisor. They are monitored online, and also written to the datastream every 10 seconds. The so-called "EPICS events" provide the status of some of the experimental hardware components. This information can then be correlated to the physics data taken around the same time. Approximately every thirty seconds, a long list of EPICS variables is inserted into the datastream. This list contains readouts of the beam current, the beam position, the magnetic fields of the spectrometer magnets, the high voltage of the detector PMTs, etc. For hardware information needed on a more frequent time-scale, a subset of the EPICS variables are read out approximately every five seconds. This subset includes the beam position and beam current. The archived EPICS data are used, for example, to study the magnetic fields of the spectrometer magnets as a function of time 
over the course of a run. These data also allow pinpointing the time during a run when a piece of equipment began to malfunction. 


\section{Chapter 3}

\section{DATA ANALYSIS}

\subsection{Overview}

Approximately 40 GBytes of data were collected during the two month running period of this experiment. These data were analyzed using the Hall A data analysis program ESPACE (Event Scanning Program for hall A Collaboration Experiments). From this analysis, elastic $e-p$ calibration events and elastic $e$ - $d$ events were identified. These events, along with other measured and calculated factors, were used to determine both elastic $e-p$ cross sections and elastic $e-d$ cross sections as a function of $Q^{2}$. The measured $e-p$ cross sections were compared to a fit of the previous world data. The results of this comparison can be seen in Section 3.12. The deuteron elastic structure functions $A\left(Q^{2}\right)$ and $B\left(Q^{2}\right)$ were extracted from the measured elastic $e-d$ cross sections. The final results of our measurements of both $A\left(Q^{2}\right)$ and $B\left(Q^{2}\right)$ are presented in Chapter 4 .

\section{$3.2 \quad$ ESPACE}

The Hall A event analyzer, ESPACE [28], was adapted from an analyzer developed at Mainz by E. Offermann, and was used to analyze all of the data taken during this experiment. The input of ESPACE consists of the raw data file (which is the 
output of CODA), along with a header file (which contains run-specific information such as spectrometer magnetic fields), a detector map file (which contains the correspondence between the event readout electronics and the physical detector outputs) and a database file (which contains basic calibration constants of the detectors, such as gains, offsets, physical positions, etc.). Using these, it calculates the focal plane vertex for each detected particle trajectory $\left(x_{f p}, y_{f p}, \theta_{f p}, \phi_{f p}\right)$. Using the spectrometer magnetic matrix elements (see Section 3.3), the coordinates of a particle trajectory at the focal plane are traced back through the spectrometer to the target to obtain the interaction vertex $\left(\theta_{t g}, \phi_{t g}, y_{t g}, \delta p / p\right)$. The output of ESPACE consists of ntuples and histograms for the analyzed events, optionally cut with various logical conditions. These outputs can be produced for all levels of the analysis, i.e. from the raw ADC and TDC information, to the position of an event within the detectors, to the measured momentum of a particle.

The goal of the analysis was to identify elastic electron-deuteron (for the calibration runs, to identify elastic electron-proton) coincidence events and determine their precise kinematics. Events were first separated by type, i.e. coincidence or single arm. Next, coincidence events with a narrowly defined time difference between the triggers of the two spectrometers were identified, reducing the number of accidental coincidence events. The events that survived these cuts were further conditioned by requiring minimal signals on the HRSE Čerenkov and shower counters. These detector cuts ensured that the particle detected in the HRSE was an electron (except for inefficiencies). The events that remained after these cuts were the elastic electron-deuteron (electron-proton) coincidence events, from which $A\left(Q^{2}\right)$ and $B\left(Q^{2}\right)$ could be extracted.

\subsection{Spectrometer Optics Calibration}

The two Hall A high resolution spectrometers were commissioned between May 1996 and April 1997. During this commissioning, the optical properties of the spectrometers were studied via electron scattering from a thin ${ }^{12} \mathrm{C}$ target. The 


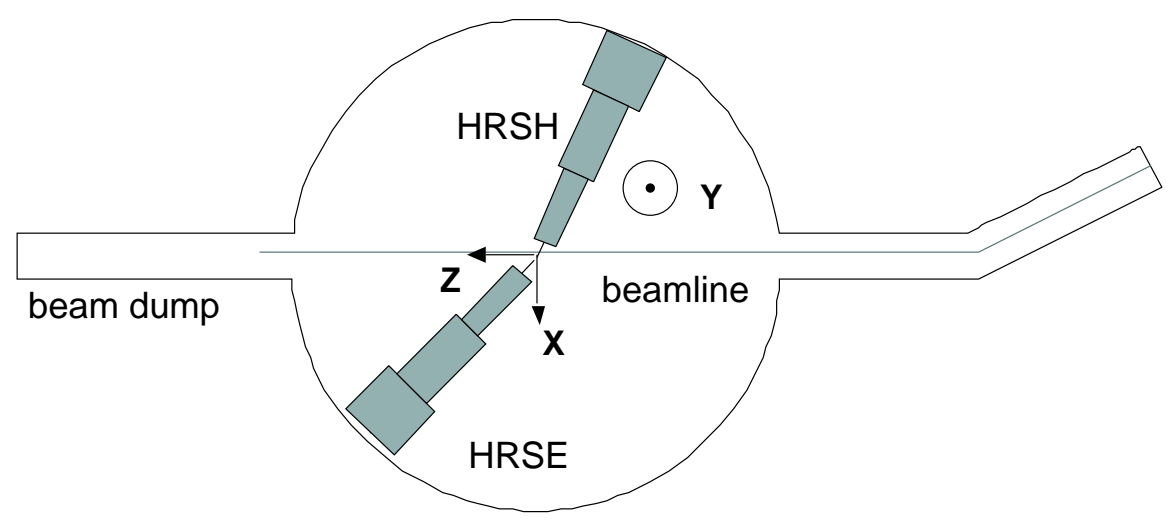

FIG. 27: Hall A Laboratory Coordinate System (top view).

sieve slit was used for these calibrations because it provides a set of trajectories with known input coordinates. ESPACE was used to analyze the sieve slit data and thus derive the spectrometer optical matrix elements. These optical matrix elements are used to reconstruct the interaction point at the target from the positions of the detected particles at the focal plane. To specify the optical properties of the spectrometers, five coordinate systems have been defined: a laboratory coordinate system, a target coordinate system and three coordinate systems linked to the detector packages of the spectrometers. These coordinate systems are described in [28] and are summarized below.

- Hall A Laboratory Coordinate System (HLCS)

The hall coordinate system is a right handed coordinate system whose origin is defined by the intersection of the electron beam with the vertical axis of the target system. The incident beam direction is defined to be the positive $z$-axis, while positive $y$ is vertically upward as defined by gravity (see Figure 27). The spectrometer's central angles are defined in this coordinate system.

- Target Coordinate System (TCS)

Each spectrometer has its own TCS. The $z_{t g}$-axis is defined by a line that is 


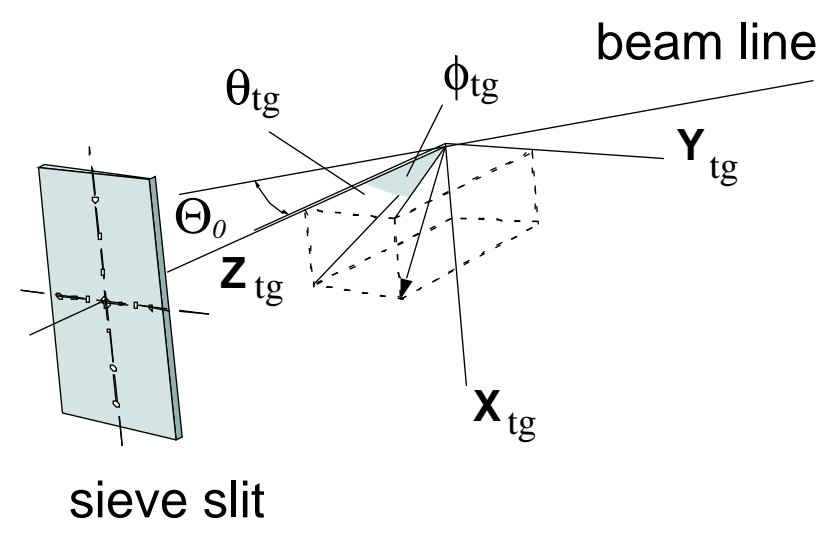

FIG. 28: Hall A Target Coordinate System.

perpendicular to the spectrometer's sieve slit, going through the midpoint of the central sieve slit hole (see Figure 28). The origin of this coordinate system is along this $z_{t g}$-axis, at a distance $Z_{0}$ from the sieve slit, at the center of rotation of the spectrometers. Ideally, the origin of the TCS should coincide with the origin of the HLCS. The $x_{t g}$-axis is parallel to a line going through the center sieve slit holes and points downward. The tangents of the out-of-plane angle $\left(\theta_{t g}\right)$ and the in-plane angle $\left(\phi_{t g}\right)$ are given by $\frac{d x_{t g}}{d z_{t g}}$ and $\frac{d y_{t g}}{d z_{t g}}$ respectively.

- Detector Coordinate System (DCS)

The DCS is defined in relation to the VDCs. The origin of this system is defined to be the intersection of wire 184 in the first (VDC1 U1) plane with the vertical projection of wire 184 in the second (VDC1 V1) plane onto the first plane (see Figure 29). From this origin, the $x_{d e t}$-axis points along the long symmetry axis (i.e. the dispersive direction) of the lower VDC, the $y_{d e t}$-axis is parallel to the short symmetry axis (i.e. the transverse direction) of the lower VDC and the $z_{\text {det }}$-axis points vertically upward. The quantities $\tan \theta_{\text {det }}$ and $\tan \phi_{\text {det }}$ are given by $\frac{d x_{\text {det }}}{d z_{\text {det }}}$ and $\frac{d y_{d e t}}{d z_{\text {det }}}$ respectively. From the intersection points of the particle track with the four VDC wire planes, 
the location of an event in the detector coordinate system can be extracted [28].

- Transport Rotated Coordinate System (TRCS)

The TRCS is obtained by rotating the DCS by $45^{\circ}$ ( $\rho_{0}$ in subsequent equations) around the $y_{d e t}$-axis so that the new $z$-axis $\left(z_{\text {trcs }}\right)$ direction is along the spectrometer central ray (see Figure 30). This axis is also perpendicular to all the detectors that follow the VDCs, so that $x_{\text {trcs }}$ and $y_{\text {trcs }}$ are respectively along the dispersive and transverse directions of those detectors.

- Focal Plane Coordinate System (FPCS)

The FPCS is obtained by rotating the DCS by an angle $\rho$ around the $y_{d e t^{-}}$ axis so that the new $z$-axis direction is along the local central ray (see Figure 31). The local central ray is the ray at the focal plane which, for a given $\delta p / p$ (the momentum relative to the central momentum of the spectrometer, i.e. $\left.\delta p / p=\frac{p-p_{0}}{p}\right)$, has $x_{t g}, y_{t g}, \theta_{t g}, \phi_{t g}=0$.

Finally, the coordinates and angle tangents of the particle track at the focal plane can be written as [28]:

$$
\begin{aligned}
x_{f p} & =\cos \rho_{0}\left(x_{\text {det }}-x_{\text {offset }}\right) \\
y_{f p} & =y_{\text {det }}-y_{\text {offset }} \\
\tan \theta_{f p} & =\frac{\tan \theta_{\text {det }}-\tan \rho}{1-\tan \theta_{\text {det }} \tan \rho} \\
\tan \phi_{f p} & =\frac{\tan \phi_{\text {det }}^{\prime}}{\cos \rho-\sin \rho \tan \theta_{\text {det }}} \\
\tan \phi_{\text {det }}^{\prime} & =\frac{\tan \phi_{\text {det }}-\tan \phi_{\text {offset }}}{1+\tan \phi_{\text {det }} \tan \phi_{\text {offset }}}
\end{aligned}
$$

where the offset terms take care of any misalignments in the VDC package and the $\theta_{\text {offset }}$ term has been absorbed in the angle $\rho$.

Given the coordinates $\left(x_{f p}, y_{f p}, \theta_{f p}\right.$ and $\left.\phi_{f p}\right)$ of an event at the focal plane, we can use the spectrometer properties to calculate the reaction vertex quantities 


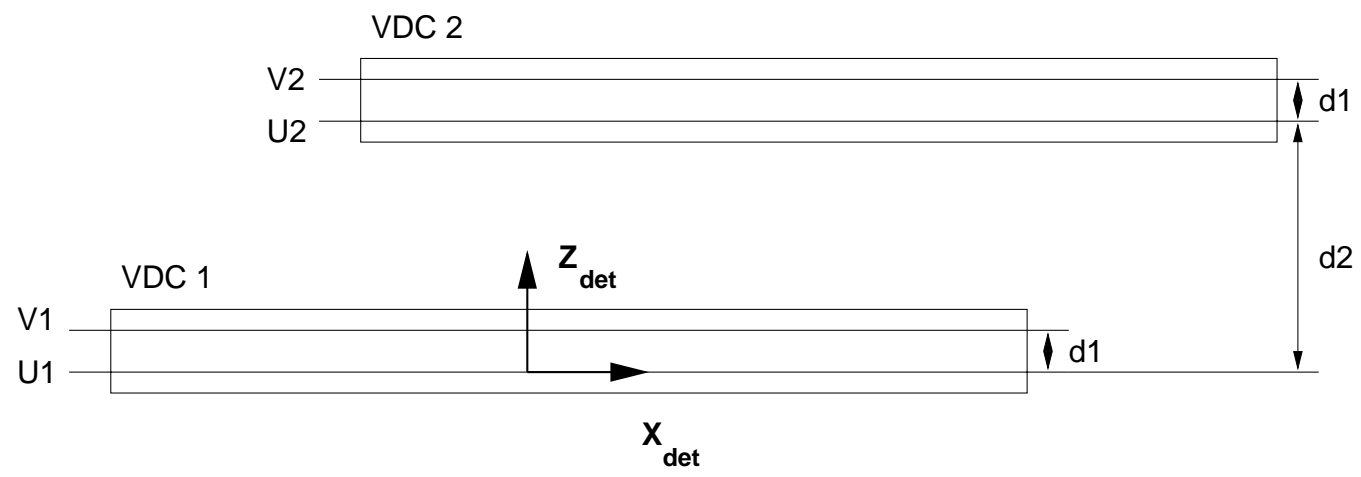

Side View

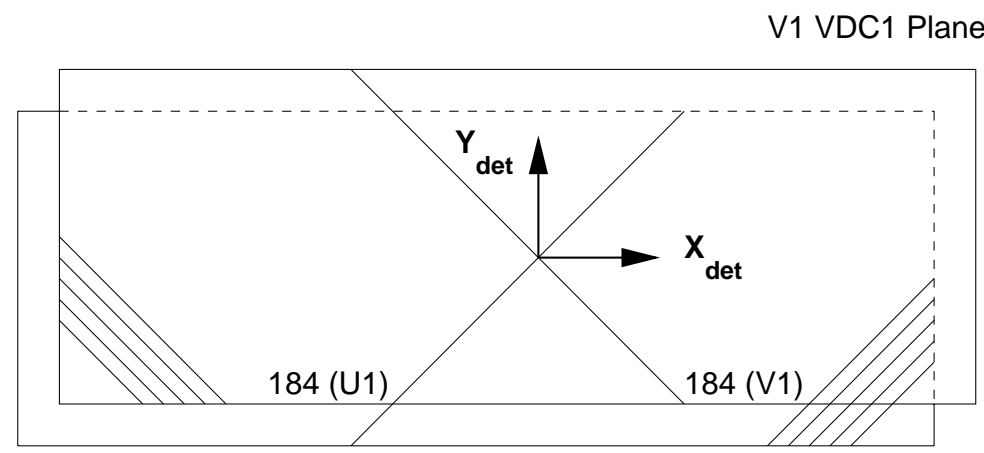

U1 VDC1 Plane

Top View

FIG. 29: Hall A Detector Coordinate System. 

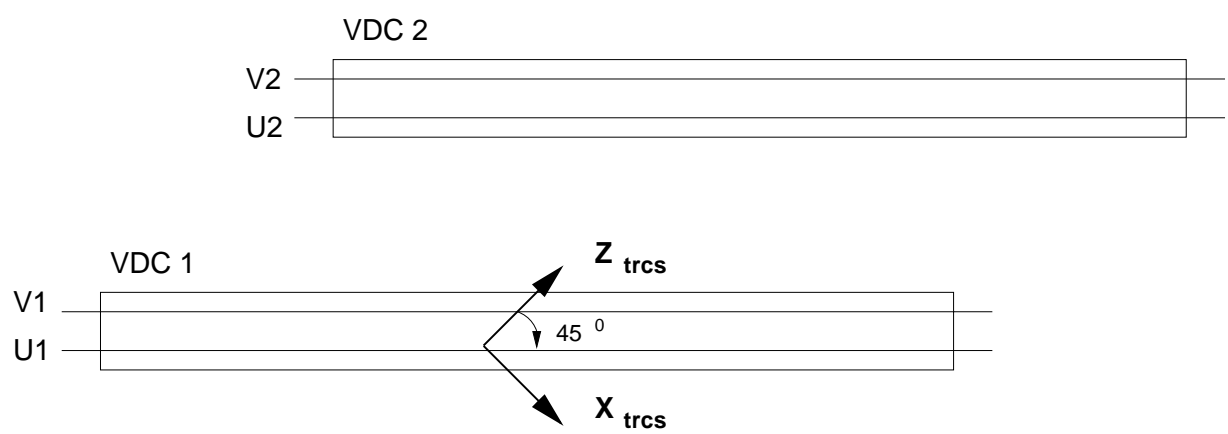

Side View

FIG. 30: Transport Rotated Coordinate System.

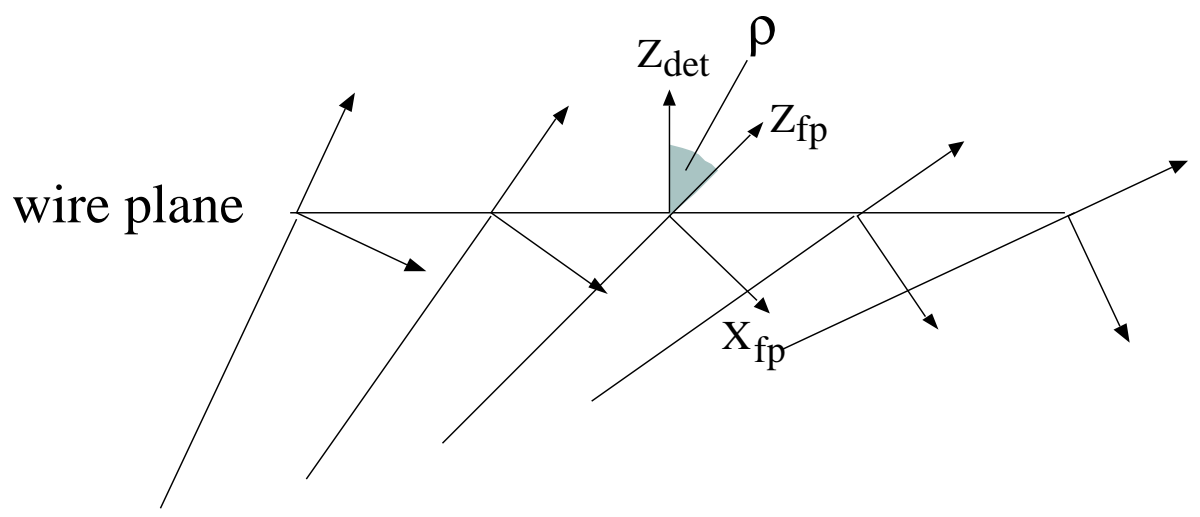

FIG. 31: Focal Plane Coordinate System. 
at the target: $x_{t g}, y_{t g}, \tan \theta_{t g}, \tan \phi_{t g}$ and $\delta p / p$. Since there are four measured quantities at the focal plane (two positions and two angles) and five unknowns at the target, some constraints have to be put on the target variables. The usual choice is to constrain the $x_{t g}$ variable to zero. This was accomplished during the commissioning by keeping the beam to within $100 \mu \mathrm{m}$ of the origin of the TCS. With a rastered beam where the $x_{t g}$ position varies by several millimeters, corrections must be made to the optical matrix elements [29]. The focal plane coordinates are linked to the target coordinates by a set of tensors $D_{i j k l}, T_{i j k l}$, $Y_{i j k l}$ and $P_{i j k l}$ :

$$
\begin{aligned}
\delta & =\sum_{i j k l} D_{i j k l} x_{f p}^{i} \tan ^{j} \theta_{f p} y_{f p}^{k} \tan ^{l} \phi_{f p} \\
\tan \theta_{t g} & =\sum_{i j k l} T_{i j k l} x_{f p}^{i} \tan ^{j} \theta_{f p} y_{f p}^{k} \tan ^{l} \phi_{f p} \\
y_{t g} & =\sum_{i j k l} Y_{i j k l} x_{f p}^{i} \tan ^{j} \theta_{f p} y_{f p}^{k} \tan ^{l} \phi_{f p} \\
\tan \phi_{t g} & =\sum_{i j k l} P_{i j k l} x_{f p}^{i} \tan ^{j} \theta_{f p} y_{f p}^{k} \tan ^{l} \phi_{f p}
\end{aligned}
$$

During the spectrometer commissioning, these tensors were determined via elastic scattering from a thin carbon foil using the sieve collimator in front of the spectrometers. A full listing of the spectrometer tensor elements and plots of the optimized momentum and position can be found in References [30] and [31].

\subsection{Beam Energy Determination}

For this experiment, the energy of the electron beam was determined through the study of coincidence elastic electron-proton scattering. For the $\mathrm{H}\left(e, e^{\prime} p\right)$ reaction, the incident beam energy is given by [32]

$$
E_{i}=M_{p}\left(\cot \frac{\theta_{e}}{2} \cot \theta_{p}-1\right)+E_{\text {loss }},
$$


where $M_{p}$ is the mass of the proton, $\theta_{e}$ is the electron scattering angle, $\theta_{p}$ is the proton angle relative to the incident beam direction and $E_{\text {loss }}$ is the energy loss of the electron before scattering (i.e. energy loss up to the reaction vertex).

The angles $\theta_{e}$ and $\theta_{p}$ are functions of the central angles of the spectrometers $\left(\theta_{0 e}\right.$ and $\left.\theta_{0 p}\right)$ and the angles of the particles relative to the spectrometer central axes [28]:

$$
\theta_{e}=\arccos \frac{\cos \theta_{0 e}-\phi_{t g e} \sin \theta_{0 e}}{\sqrt{1+\theta_{t g e}^{2}+\phi_{t g e}^{2}}}
$$

for the electron spectrometer and

$$
\theta_{p}=\arccos \frac{\cos \theta_{0 p}-\phi_{t g p} \sin \theta_{0 p}}{\sqrt{1+\theta_{t g p}^{2}+\phi_{t g p}^{2}}}
$$

for the hadron spectrometer, where $\theta_{0 p}$ is defined to be negative and $\theta_{0 e}$ positive. $\theta_{t g e(p)}$ and $\phi_{t g e(p)}$ are the angles of the particle track with respect to the spectrometer central ray in the dispersive $(x)$ and transverse $(y)$ directions. A small angle approximation has been assumed in Equations (16) and (17), so that $\tan ^{2} \theta_{\text {tge }}(p)$ has been approximated as $\theta_{t g e(p)}^{2}$.

The error on the beam energy determined using this method is given by

$$
\delta E_{i}=\sqrt{\left(\frac{\delta E_{i}}{\delta \theta_{e}}\right)^{2} \delta \theta_{0 e}^{2}+\left(\frac{\delta E_{i}}{\delta \theta_{p}}\right)^{2} \delta \theta_{0 p}^{2}},
$$

where $\delta \theta_{0 e}$ and $\delta \theta_{0 p}$ are the uncertainties in the surveyed positions of the spectrometer central angles. The error on fitting the centroid of the energy distribution is negligible as reported by the PAW [33] Gaussian fitting routine. Taking the required partial derivatives of Equation (15) leads to

$$
\Delta E_{i}=\sqrt{\frac{M_{p}^{2} \cot ^{2} \theta_{p}}{4 \sin ^{4} \frac{\theta_{e}}{2}} \Delta \theta_{0 e}^{2}+\frac{M_{p}^{2} \cot ^{2} \frac{\theta_{e}}{2}}{\sin ^{4} \theta_{p}} \Delta \theta_{0 p}^{2}} .
$$


The uncertainty in the central angles of both the electron and hadron spectrometers is 0.3 milliradians from repeated surveys of their positions. For the kinematics of this experiment and the $0.3 \mathrm{mrad}$ angular uncertainties, the typical error on the determination of the central value of the beam energy was $0.1-0.2 \%$.

The reconstructed beam energy calculated using Equation (15) on an event by event basis is shown in Figure 32. The width of the distribution is consistent with multiple scattering and the finite angular resolution of the spectrometers. For this kinematics, the beam energy reported by the accelerator operators was 4424.0 $\mathrm{MeV}$. The centroid of the distribution in Figure 32, representing the calculated beam energy before energy loss is taken into account, is $4392.1 \mathrm{MeV}$. The energy loss is calculated assuming that the incident electron interacts at the center of the cryotarget, after passing through $7.5 \mathrm{~cm}$ of liquid hydrogen. For this kinematics, the energy loss is $2.7 \mathrm{MeV}$, leading to a total energy extracted from the $\mathrm{H}\left(e, e^{\prime} p\right)$ analysis of $4394.8 \mathrm{MeV} \pm 4.7 \mathrm{MeV}$, where the error is obtained from Equation (19). This energy is $0.7 \%$ lower than the nominal beam energy of $4424.0 \mathrm{MeV}$.

A total of seventeen $\mathrm{H}\left(e, e^{\prime} p\right)$ runs for the $B\left(Q^{2}\right)$ kinematics and eighteen runs for the $A\left(Q^{2}\right)$ kinematics were analyzed using this technique to extract the incident beam energy. They were all found to yield an energy below the nominal value reported by the accelerator. A plot of the normalized energy difference ((measured-nominal)/nominal) versus run number (i.e. time) is shown in Figure 33. The top half shows the energy for the $B\left(Q^{2}\right)$ kinematical points, all taken at beam energies below $1.0 \mathrm{GeV}$, and the bottom half shows the energy extracted for the $A\left(Q^{2}\right)$ points, all taken at energies above $3.0 \mathrm{GeV}$. For the $B\left(Q^{2}\right)$ runs, the average energy difference between the measured value and the nominal value reported by the accelerator is $-0.6 \%$. For the $A\left(Q^{2}\right)$ kinematics, the average energy difference is also $-0.6 \%$.

This difference between the reported accelerator energy and the measured Hall A energy has been traced to a discrepancy in the determination of the magnetic field in the arc of eight magnets that the beam passes through between the Beam Switchyard and Hall A. During this experiment, the magnetic field of the arc had not been mapped, so it was determined from the current in the magnet coils. 


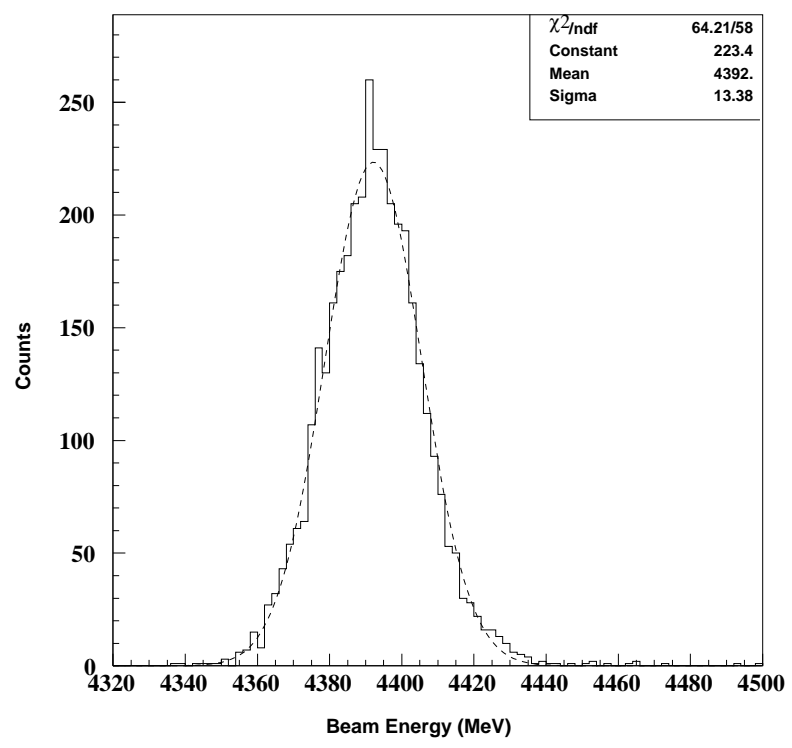

FIG. 32: Incident beam energy from $\mathrm{H}\left(e, e^{\prime} p\right)$ with nominal beam energy 4424.0 $\mathrm{MeV}$. The electron scattering angle is $\theta_{0 e}=31.17^{\circ}$ and the proton scattering angle is $\theta_{0 p}=-32.12^{\circ}$. The solid line is the data and the dashed curve is a Gaussian fit. 
About six months after this experiment, the arc magnets were removed from the arc and their fields accurately mapped. After this mapping, it was found that the field values that had been used during E91-026 to determine the energy of the beam being delivered to Hall A were approximately $0.40-0.45 \%$ too high. This means the accelerator reported nominal energy was $0.40-0.45 \%$ too high, since there is a linear dependence of the beam's momentum on the field strength. The value extracted for the beam energy is also a function of the path followed by the beam through the arc magnets, so a further correction to the nominal energy can arise. This correction was found to be approximately $0.20 \%$ for a run at the end of October (around run 835 in Figure 33), leading to the accelerator reported nominal energy being $0.6-0.7 \%$ too high. This is consistent with our measured energies during the same time frame. It was decided to apply a constant correction to the nominal accelerator reported energy of $-0.6 \%$, with an error of $\pm 0.1 \%$ to account for the spread in the measured energies.

\subsection{Total Charge Determination}

The discussion in Section 2.4.2 focused on charge extraction from the BCM signals, but the signals provided by the BCMs are only proportional to the beam current. The absolute value of the current is provided by the Unser monitor. Unfortunately, the zero offset of the Unser monitor drifts on the scale of minutes, so the more stable BCMs are used as the continuous current monitor. A calibration procedure has been developed to calibrate the output of the BCM's to that of the Unser monitor.

The calibration procedure consists of a series of beam current steps (cf. Fig. 34), typically from 0-100 $\mu \mathrm{A}$. During each current calibration step, the current remains at zero for one minute. During this time, the Unser monitor signal is recorded. The average of this value represents the average zero offset of the Unser monitor, denoted $I_{0}$. Next the current is stepped to some maximum value (typically $\geq 100 \mu \mathrm{A}$ ) as quickly as possible. The current remains at this maximum for one minute, during which time the outputs of the Unser and the two BCMs are 

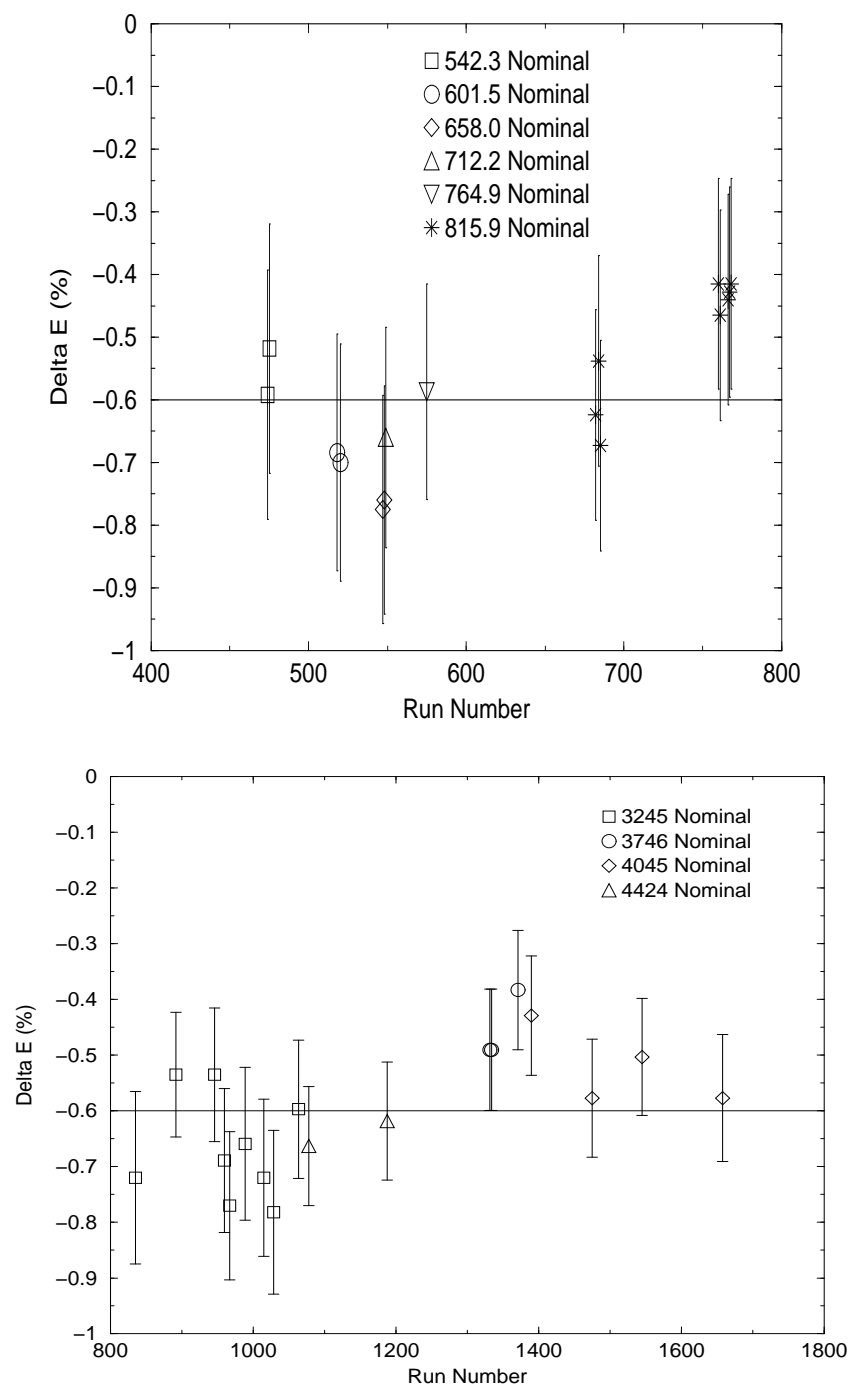

FIG. 33: Incident beam energy versus run number (time). The runs are all elastic $e-p$ runs. For the top plot, which are the $\mathrm{H}\left(e, e^{\prime} p\right)$ runs taken for the $B\left(Q^{2}\right)$ kinematics, the average energy difference is $-0.6 \%$, marked with the solid line. The bottom plot is the energy extracted from the $A\left(Q^{2}\right)$ kinematics. The average energy difference is also $-0.6 \%$. 


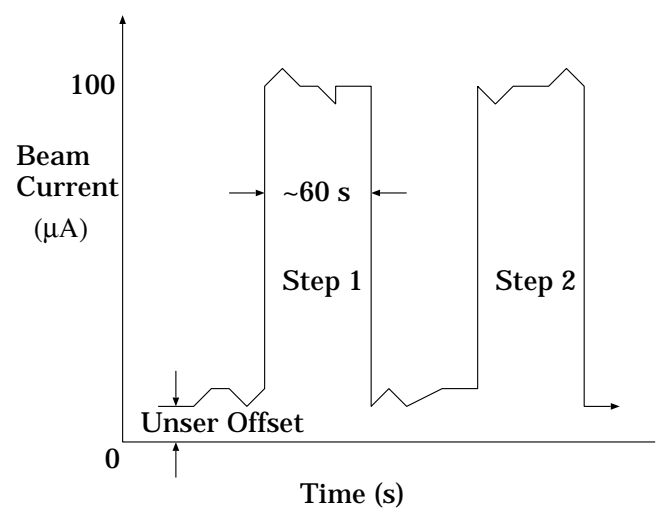

FIG. 34: Current calibration schematic.

recorded. Denoting the output of the Unser as $I_{u}$ and the output of the BCM's as $V_{1}$ and $V_{2}$ (for the upstream and downstream BCM respectively), the output of the BCM's can be related to the current measured by the Unser monitor as:

$$
I_{u}-I_{0}=A_{1(2)} \times V_{1(2)}
$$

where $A_{1(2)}$ is the calibration coefficient for the upstream (downstream) BCM. This calibration procedure continues for four more steps in current (from zero to maximum). At the end of the procedure an average is taken of the calibration coefficients computed during each step, and this becomes the best estimate of the calibration coefficient, until the next current calibration. The standard deviation is also reported for the average calibration coefficient.

During the analysis of Experiment 89-003 (the first Hall A experiment), a calculation was made of the magnitude of the expected error of the calibration coefficients $A_{1}$ and $A_{2}$ [34]. The expected error was calculated to be on the level of $0.3 \%$, while the average standard deviation on all of the calibration coefficients taken during this experiment was on the order of $1.2 \%$. The cause of this difference between the expected error and the measured error was found to be a problem with 
the algorithm used by the calibration procedure. Namely, the Unser and BCM readings were sometimes found to get out of sync during the part of the calibration sequence where the current went from 0 to maximum or vice versa, so that, for example, one meter would be reading the maximum current and the other would still be reading the zero current. Assuming that this error is random and can occur in both directions equally, the calibration coefficients should scatter around the true value. Therefore, the standard deviation of the calibration coefficients is taken to be the error on the calibration coefficient (and therefore the current and the charge).

\subsection{Electron and Recoil Tracking}

The vertical drift chambers (VDCs) of the Electron and Hadron spectrometers are filled with a gaseous mixture of argon and ethane (50\% each). When a charged particle passes through the chambers, it ionizes the gas and the freed electrons drift along the electric field lines formed by the high voltage planes and signal wires (see Section 2.7.1 for a description of the VDC hardware). In the vicinity of the signal wires, the electric field strength increases and the electrons gain enough energy between collisions to ionize additional gas molecules. In turn, those electrons can ionize more gas molecules, and so on, forming an avalanche of electrons incident on the wires. At the same time, the positive ions are drifting away from the signal wires, leading to an appreciable negative signal on the wires. A TDC, which is stopped by the event trigger, is used to infer the elapsed time between the initial ionization and an above threshold signal being induced on a signal wire. This drift time, combined with the electron drift velocity, yields the drift distance (see Reference [31] for details).

Typically, a charged particle induces a signal on three to six wires as it passes through the chamber. As illustrated in Figure 35, the particle's path through the VDC is reconstructed by fitting the perpendicular distances to each hit wire with a tracking algorithm. The tracking algorithm chooses tracks via a goodness-of-fit test, so the first track with a $\chi^{2}$ within acceptable limits is chosen as the track 


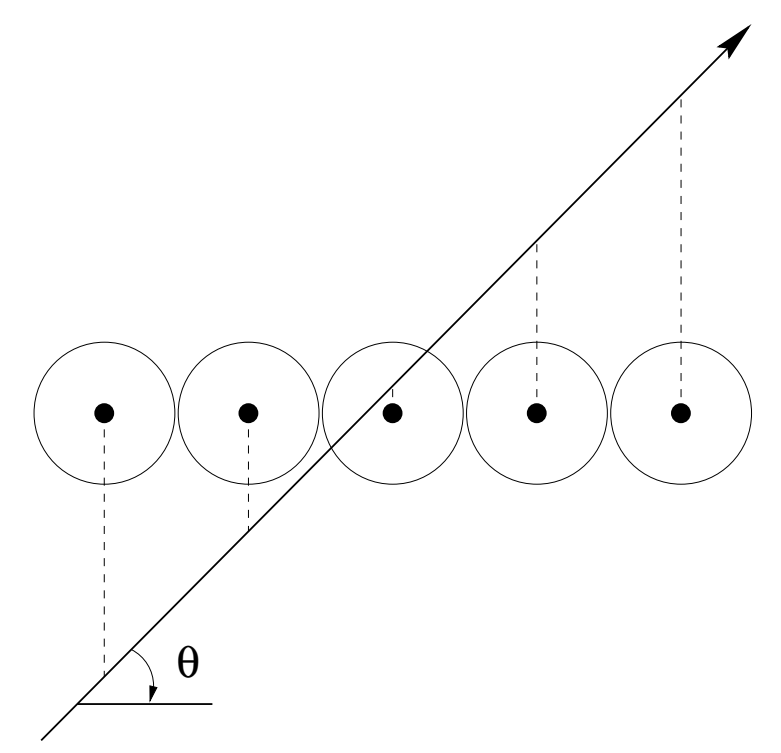

FIG. 35: A typical trajectory in one of the VDC wire planes. The perpendicular distances (dashed lines) to each hit wire are fit to reconstruct the particle's path (solid line) through the chambers. The circles indicate the boundary between the regions of uniform electric field and the regions of radial electric field. 
of the particle which generated the trigger. This track is later subject to more constraints during analysis: it has to point back to the target and forward to the shower detectors, etc. Since there are four wire planes (2 VDCs per spectrometer, each with two wire planes), two positions and two angles can be obtained from the chambers, allowing determination of $\left(x_{f p}, y_{f p}, \theta_{f p}, \phi_{f p}\right)$.

\subsection{Electron Identification}

The HRSE Čerenkov and Shower counters were used to distinguish between electrons and negatively charged pions. Electrons were required to produce a minimum signal in the Čerenkov counter. Also, the ratio of the total energy deposited in the shower counter to the tracked momentum of the particle ( $\frac{E^{\prime}}{P^{\prime}}$, or EP Ratio) had to be greater than a minimum value.

\subsection{1 Čerenkov Analysis}

The Čerenkov counter used in this experiment (see Section 2.7.3) was a threshold counter, where an electron with a momentum above $17 \mathrm{MeV} / \mathrm{c}$ or a pion with a momentum above $4.8 \mathrm{GeV} / \mathrm{c}$ could produce Cerenkov light. Since the threshold momentum for pions is above the maximum accepted momentum of the spectrometer, pions could only give a Čerenkov signal through the production of knock-on electrons.

The ADC spectrum for a single phototube of the Čerenkov counter is shown in Figure 36. The sharp pedestal peak (the PMT signal when no light is present) is seen around channel 500. After the pedestal, the single and double photoelectron peaks (the PMT signals for one and two photoelectrons being liberated from the PMT photocathode by the incident light) can be seen. Next is a broad distribution that corresponds to the PMT signal for varying numbers of photoelectrons. The average number of photoelectrons emitted for the Cerenkov phototubes, Tube 7 excluded, was approximately eight. Tube 7 was coated with the wavelength shifter p-Terphenyl, which shifted some of the low wavelength Cerenkov light into the 


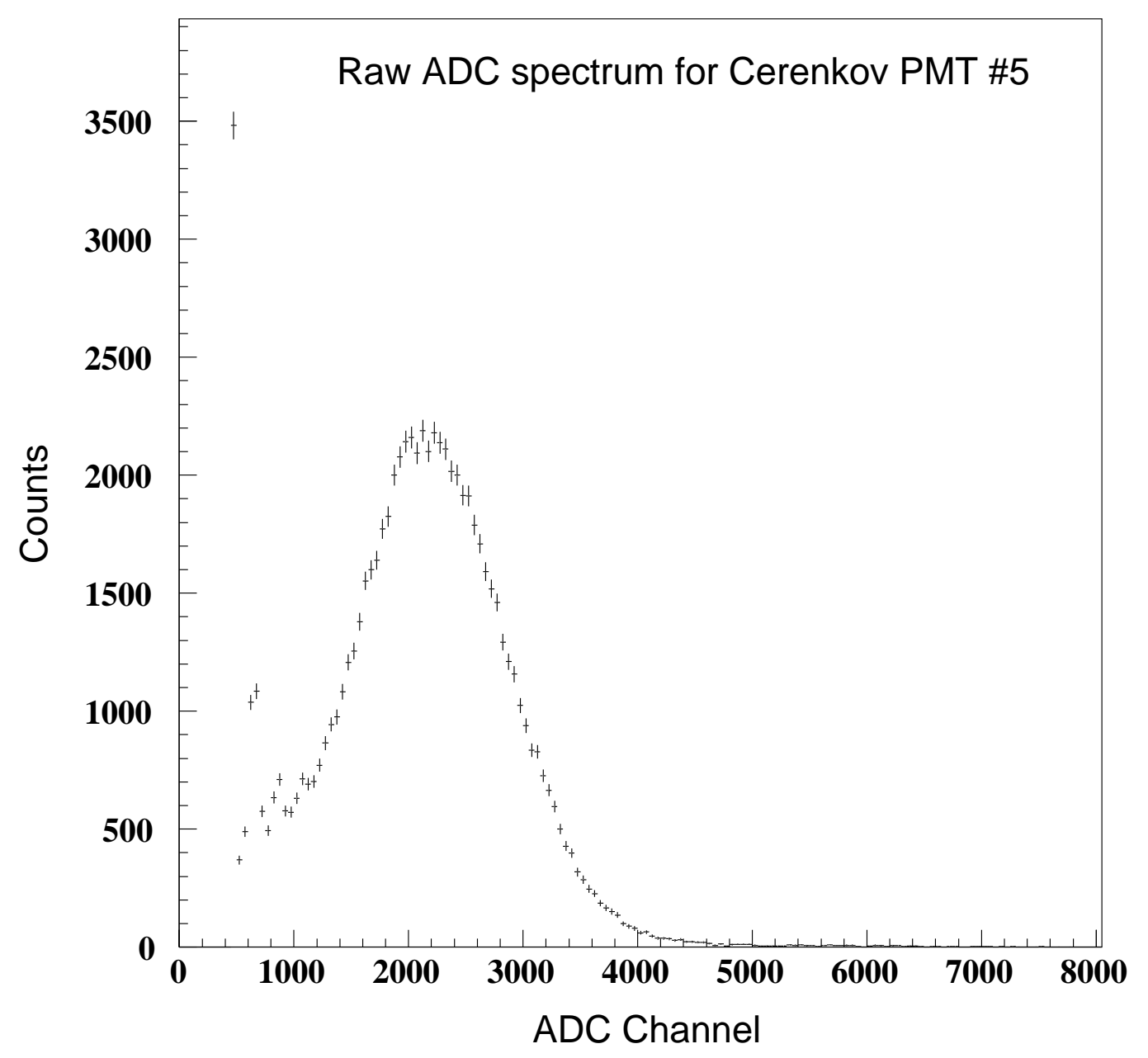

FIG. 36: ADC spectrum for a typical PMT on the EARM gas Čerenkov counter. The spike around channel 500 is the pedestal, which is the PMT signal when no light is present. The peak around channel 600 is the single photoelectron peak, while the peak around channel 800 is the two photoelectron peak. 


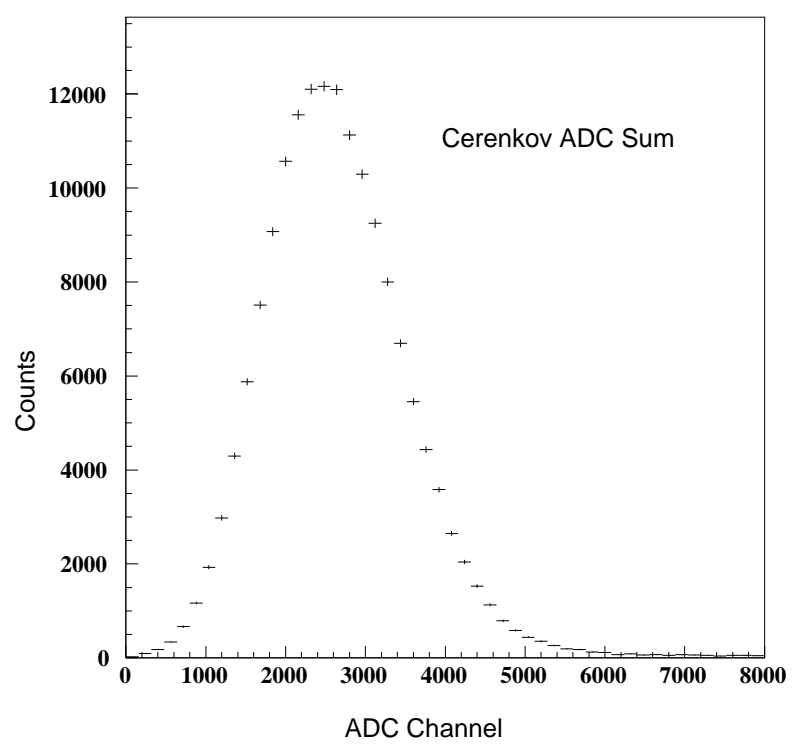

FIG. 37: Sum of the ADC spectra for all ten phototubes of the Cerenkov detector. A good electron event was required to have an ADC sum above Channel 500.

sensitive range of the PMT, causing it to average approximately 13 photoelectrons emitted per event. Since the Cerenkov light emitted by the incident charged particle can strike more than one of the counter's mirrors, the sum of the ADC values for all ten PMT's is used for particle identification. A histogram of this sum accumulated over the course of a run can be seen in Figure 37. A pion passing through the detector stack can knock out electrons from the detector materials. These electrons, known as $\delta$ - or knock-on electrons, can generate a signal in the Cerenkov counter, but they are of lower energy than the electrons which were scattered from the target, so they tend to produce a signal whose ADC sum is peaked at small values. To remove these $\delta$-electron events, a cut was placed requiring particles to produce a Cerenkov ADC sum greater than a threshold value of ADC channel 500. 


\subsubsection{Shower Energy}

The total energy deposited in the shower counters is the sum of the energy deposited in both the pre-shower and shower detectors. The method of extracting the energy deposited in these two detectors is summarized below and described in detail in Reference [35].

For each event, the total energy deposited in the pre-shower and shower counters is given by the sum of the energy deposited in the cluster of blocks around the reconstructed particle track. The deposited energy is calculated by multiplying the block's signal by a calibration coefficient determined previously $\left(C_{P S i}\right.$ and $C_{S H j}$ for the $i^{t h}$ block of the pre-shower and $j^{\text {th }}$ block of the shower detector). The total shower energy, $E^{\prime}$, normalized to the tracked particle momentum, $P^{\prime}$, is given by:

$$
\frac{E^{\prime}}{P^{\prime}}=\frac{\left(\sum_{i=1}^{N_{P S}} C_{P S i}\left(A_{P S i}-P_{P S i}\right)+\sum_{j=1}^{N_{S H}} C_{S H j}\left(A_{S H j}-P_{S H j}\right)\right)}{P^{\prime}},
$$

where $A_{P S i}$ and $P_{P S i}$ are the ADC signal and pedestal for the $i^{\text {th }}$ pre-shower block and $A_{S H j}$ and $P_{S H j}$ are for the $j^{\text {th }}$ shower block. $N_{P S}$ and $N_{S H}$ represent the number of blocks around the central block that the energy is summed over (the cluster). For the pre-shower detector, a cluster is defined as 6 blocks in a 2 $\times 3$ array, while for the shower detector, a cluster is 9 blocks in a $3 \times 3$ array (see Figure 38). A pion passing through the shower detectors deposits less energy than an electron because of its larger mean free path (cf. Figure 39). To cut pions out of the data, events were required to have deposited a normalized amount of energy $\frac{E^{\prime}}{P^{\prime}}>0.6$.

The pre-shower and shower block coefficients were determined using "white spectra" runs (where the shower counter blocks were nearly uniformly illuminated with particles) with "clean" electron events. The electron calibration events were required to pass several cuts:

1. Data reconstruction in all of the detectors was successful.

2. Only one track was reconstructed in the VDCs. 


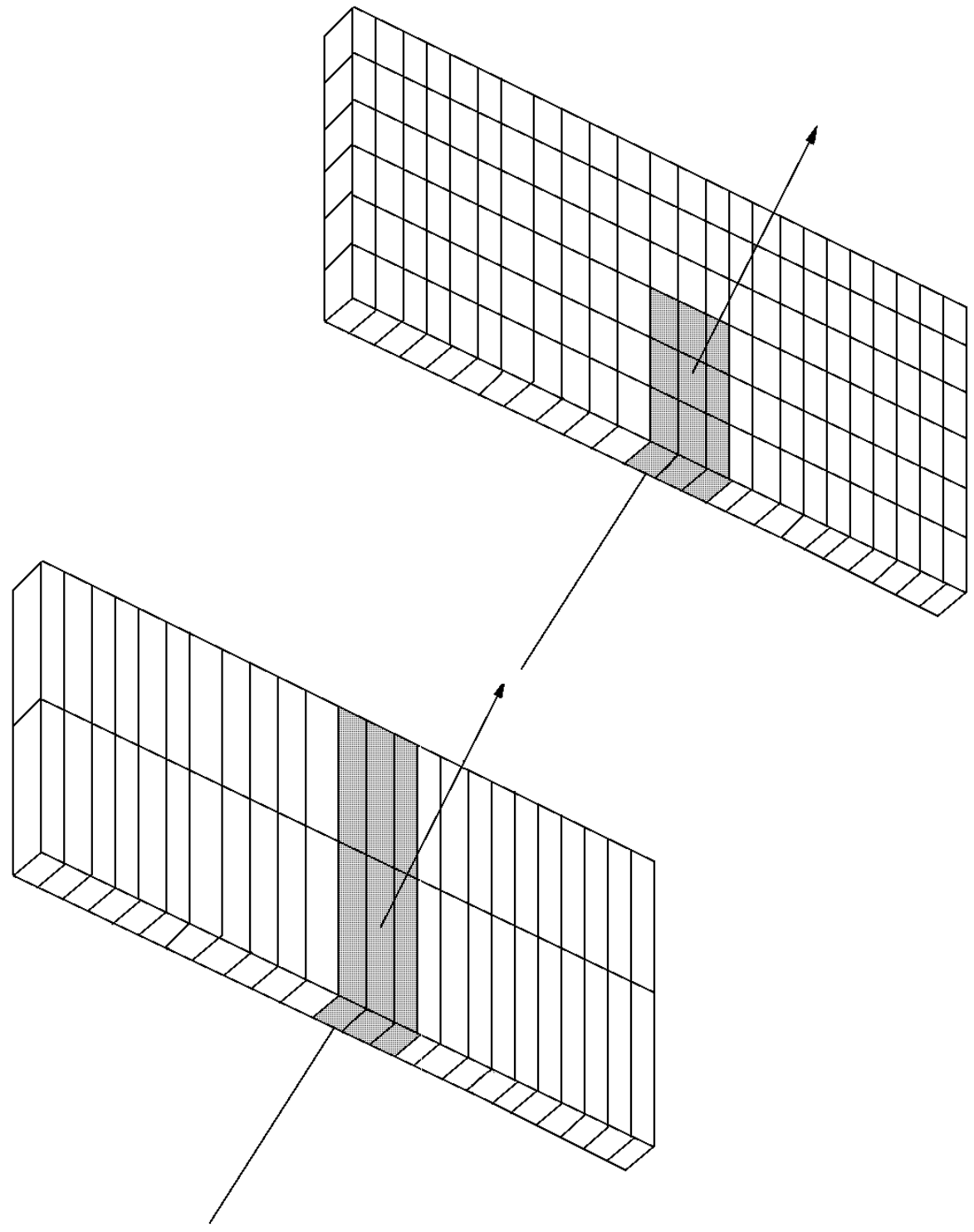

FIG. 38: Cluster of blocks (shaded blocks) considered for the pre-shower (below) and shower (above) detectors for the given particle track (arrowed line). 


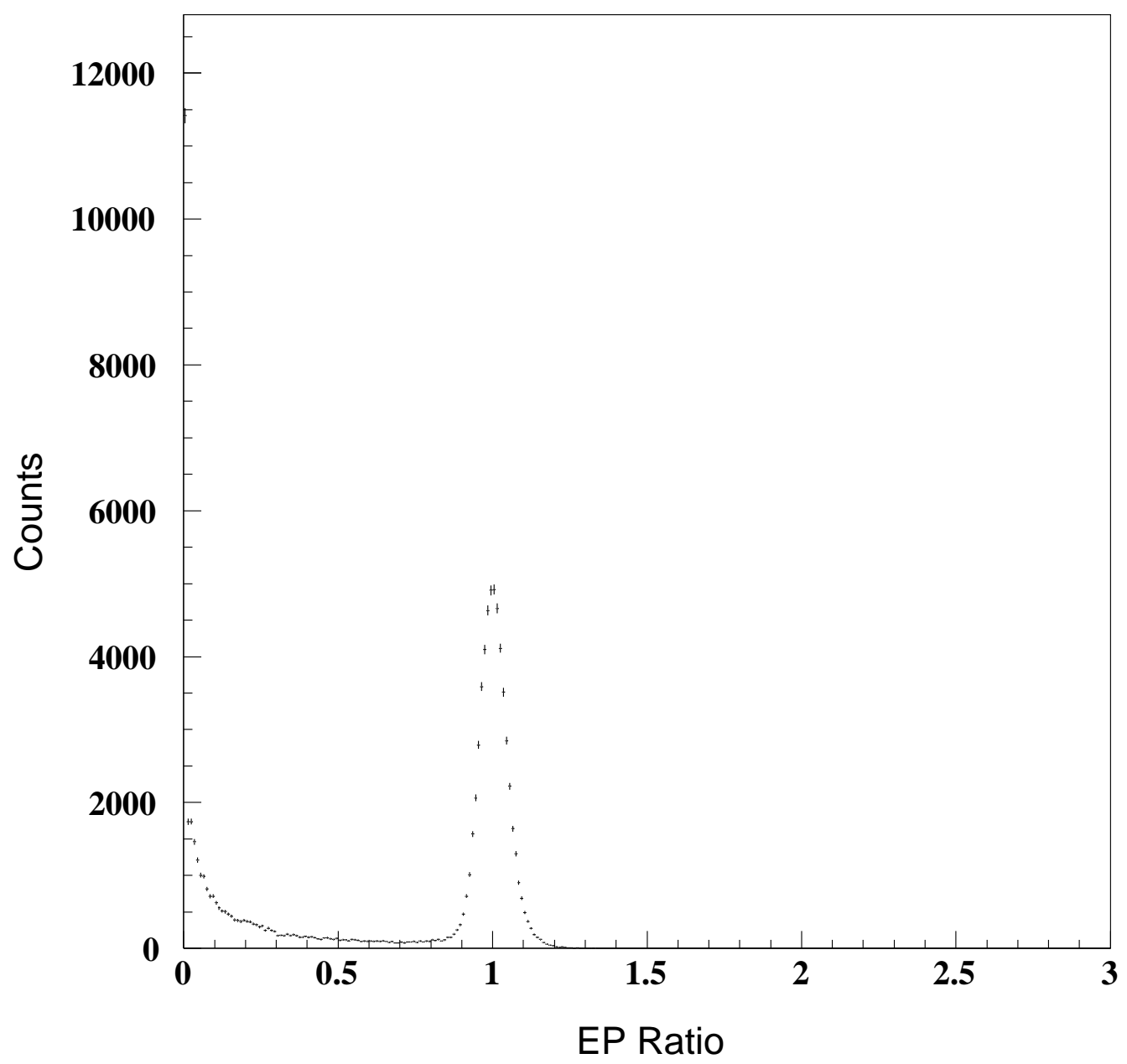

FIG. 39: Raw spectrum of the ratio of the sum of the energy deposited in both the pre-shower and shower counters to the tracked momentum of the particle (EP Ratio). A good electron event was required to have an EP Ratio greater than 0.6. 
3. The sum of the amplitudes of the gas Cerenkov signal was greater than a threshold value.

4. Only one cluster of blocks fired in each of the pre-shower and shower detectors.

For events that passed these cuts, the calibration coefficients were fitted by minimizing the functional

$$
\chi^{2}=\sum_{k=1}^{N}\left[\sum_{i \in M_{P S}^{k}} C_{P S i}\left(A_{P S i}^{k}-P_{P S i}\right)+\sum_{j \in M_{S H}^{k}} C_{S H j}\left(A_{S H j}^{k}-P_{S H j}\right)-P^{k}\right]^{2},
$$

where $N$ is the number of calibration events that passed the above cuts, $i$ represents the indices of the pre-shower blocks included in the cluster (reconstructed in the $k^{t h}$ event), $j$ represents the indices of the shower blocks included in the cluster (reconstructed in the $k^{t h}$ event), $M_{P S}^{k}$ and $M_{S H}^{k}$ respectively are the set of pre-shower and shower blocks included in the cluster for the $k^{t h}$ event and $P^{k}$ is the tracked momentum of the $k^{t h}$ event. In this way, the calibration constant for each block was determined. The total number of these constants is 144 (48 for the pre-shower and 96 for the shower detector).

\subsection{Recoil Particle Identification}

Deuterons from elastic electron-deuteron scattering events were separated from other particles in the hadron spectrometer by means of a time-of-flight cut. The coincidence time-of-flight (TOF) is the time difference between a trigger in the Electron spectrometer and a trigger in the Hadron spectrometer. For a real coincidence event where the particles originate from the same scattering, the TOF corresponds to the difference in flight times through the spectrometers between an electron and a hadron. 


\subsubsection{Coincidence Time-of-Flight}

The Coincidence TOF is measured by starting a TDC when a trigger is observed on the HRSE and stopping it when a trigger is observed on the HRSH. Real coincidences are expected to have a narrow range of coincidence times, since, for a given kinematics, the momentum range of the scattered deuterons is small. There are also random coincidences caused by uncorrelated single arm events in each spectrometer that just happen to fall within the coincidence time window. These accidental coincidences form a flat background in the coincidence TOF spectrum. In the case of electron-deuteron scattering, there is also a background of coincidences caused by protons coming from the disintegration of the deuteron. The top half of Figure 40 shows the raw coincidence time spectrum. The peak around channel 225 corresponds to true elastic electron-deuteron coincidences. This figure shows the flat background caused by uncorrelated events, and also a secondary peak caused by inelastic electron-proton coincidence events.

It is desirable to make the coincidence time peak as narrow as possible so that real coincidences are easily separated from background and the statistical uncertainty coming from accidentals within the peak time window is reduced. To do this, there are several corrections which are applied to the coincidence time calculation. The first accounts for time differences caused by where the charged particle strikes the trigger scintillator. The second accounts for differences in velocity and flight-path of the electrons and hadrons through the spectrometers. In general, the TOF for a particle traveling along the central ray of the spectrometer is given by

$$
t_{0}=\frac{l_{0}}{\beta_{0}}
$$

where $l_{0}$ is the path length along the central ray and $\beta_{0}$ is the velocity of a particle traveling along the central ray. For a particle whose path differs from the central ray by $\Delta l$, the flight-time would be

$$
t=\frac{l_{0}+\Delta l}{\beta}
$$


where $\beta$ is the velocity of the particle and the path difference $\Delta l$ can be calculated from the particle's position in the focal plane. The correction to the TOF is then given by

$$
\Delta t=t-t_{0}=\frac{l_{0}+\Delta l}{\beta}-\frac{l_{0}}{\beta_{0}} .
$$

The effect of applying these corrections on the TOF spectrum is shown in the bottom half of Figure 40. True coincidences are now found in the narrow peak centered around channel 214. The secondary proton peak is still visible in this figure since the spectrum shown is uncut on detector signals which allow particle identification (PID). The effect of cutting on a minimum signal from the Čerenkov and a minimum EP Ratio from the shower counters is shown in Figure 41. The background has been reduced to a minimum and the events within the surviving peak are the true elastic $e-d$ coincidence events.

\subsection{Corrections to the Experimental Data}

There are several effects that would reduce the extracted number of coincidence events and yield an artificially low cross section. The first is that of detector and trigger inefficiencies, which means an inefficiency in the shower counters, the Čerenkov or in the scintillators or trigger setup itself. Deadtime and prescaling also cause a reduction of events. Deadtime refers to a potentially good event not being recorded because the data acquisition was busy. Raw events are prescaled at the Trigger Supervisor, which throws away a fraction of potentially good events. Another effect that could decrease the number of coincidence events is re-scattering or absorption of the recoiling proton or deuteron on its way out of the cryotarget, through the scattering chamber exit window or in the windows of the spectrometer. A "boiling" correction is also applied to the data, since the density of the target varies as a function of the incident beam current. These corrections are discussed in detail in the sections that follow. 

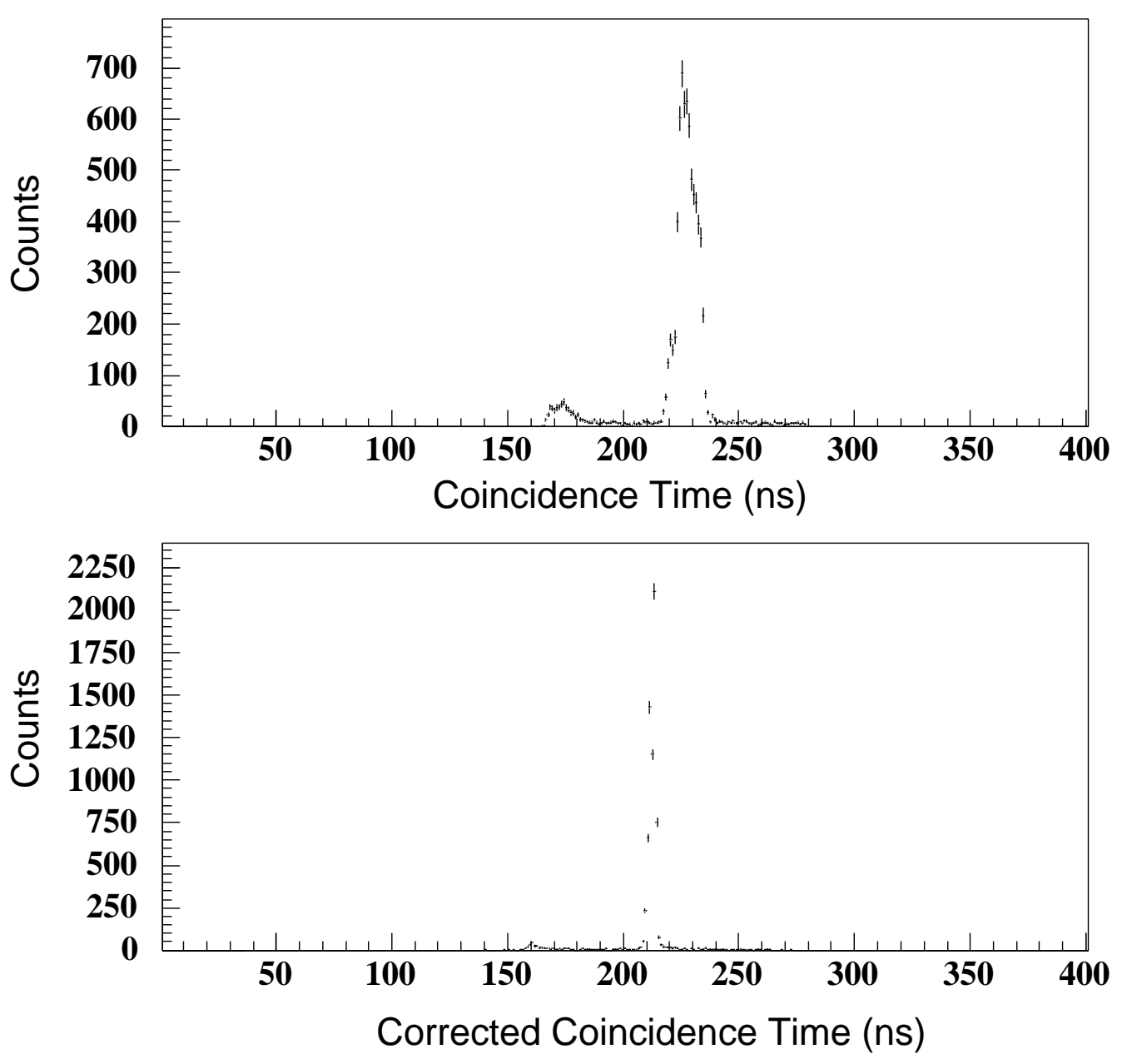

FIG. 40: Raw coincidence time (top) and corrected coincidence time (bottom) spectra from an $e$ - $d$ run at $E_{\text {beam }}=3.245 \mathrm{GeV}$ and $Q^{2}=1.22(\mathrm{GeV} / \mathrm{c})^{2}$. The raw coincidence time spectrum shows two peaks; one from elastic electron-deuteron scattering events (centered around channel 225) and one from inelastic electronproton events coming from the breakup of the deuteron (far left). The time difference between these peaks corresponds to the TOF difference between protons and deuterons through the hadron spectrometer. The bottom spectrum shows the effect of corrections applied to the coincidence time calculation. These corrections are described in the text. 


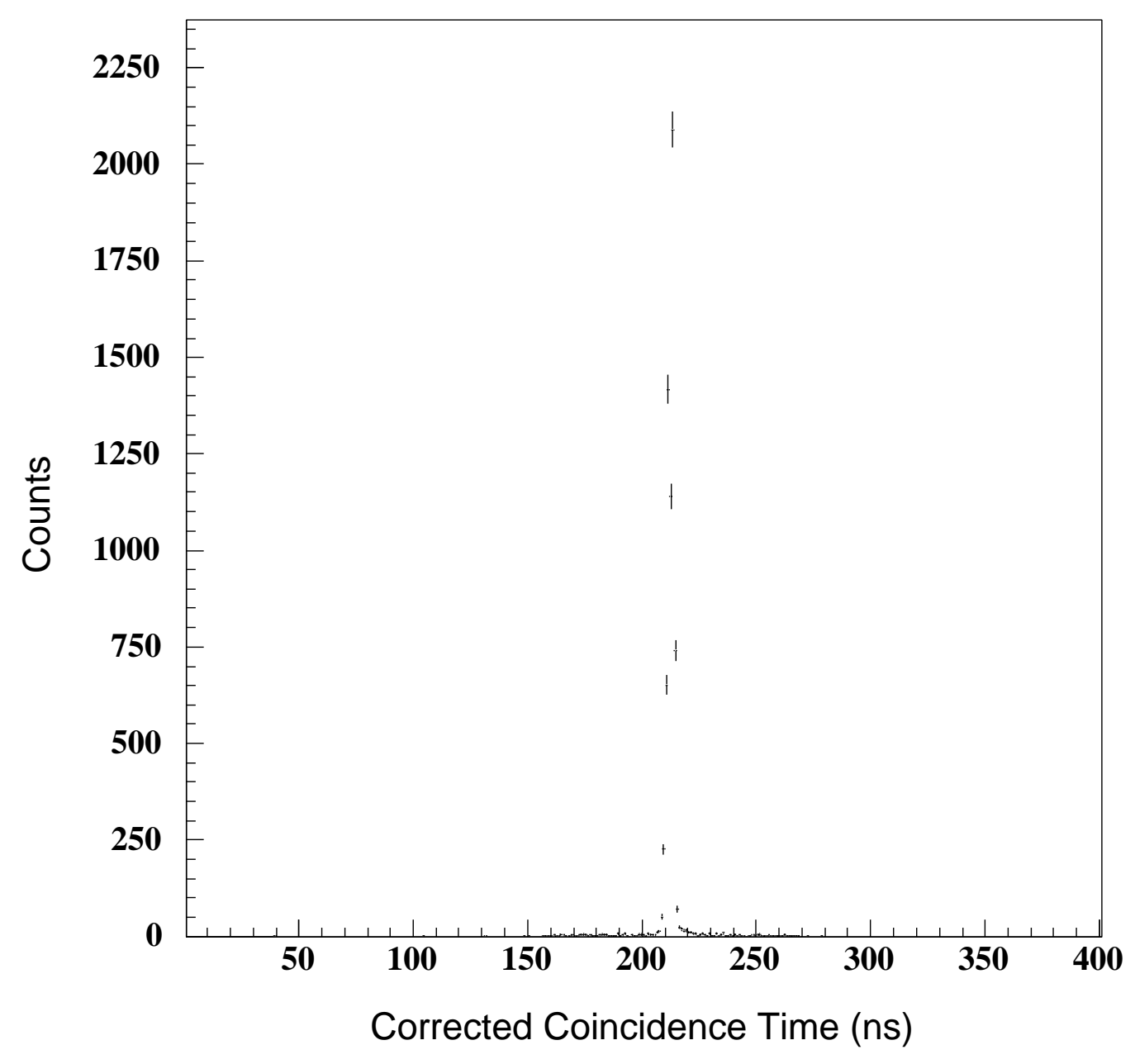

FIG. 41: Corrected coincidence time spectrum with detector cuts applied. The cuts include a minimum signal in Čerenkov counter and a minimum $E^{\prime} / P^{\prime}$ ratio from shower counters. The events within the peak centered around Channel 214 are the true elastic $e-d$ coincidence events. 


\subsubsection{Detector and Trigger Inefficiencies}

A correction is made to the data to account for inefficiencies in the detectors and the trigger logic. This correction is denoted as $C_{d e t}$ in subsequent sections.

\section{Trigger and Scintillator Inefficiencies}

The inefficiency of the trigger is a combination of inefficiencies in the scintillators that form the trigger and inefficiencies in the trigger logic itself. The trigger inefficiency of each spectrometer for coincidence events is determined by taking the ratio of coincidence events that gave a trigger in the other spectrometer to the coincidence events that gave a trigger in both spectrometers. The events that gave a trigger in both spectrometers were required to have a good raw coincidence time, a Čerenkov ADC sum between channels 500 and 8000 and an EP ratio (the ratio of the energy deposited in the shower counter to the tracked momentum of the particle) between 0.50 and 2.50 for the backward angle $\left(B\left(Q^{2}\right)\right)$ data and between 0.65 and 2.50 for the forward angle $A\left(Q^{2}\right)$ data.

For proton coincidence measurements, the electron arm trigger inefficiency averaged $1.5 \%$ for the backward angle data and $1.0 \%$ for the forward angle data. The hadron arm inefficiency averaged 1.0\% for the backward angle data and 1.8\% for the forward angle data. A systematic error of $0.3 \%$ is applied to these results to account for the spread in the measurements. For deuteron coincidence measurements, the electron arm efficiency was taken to be the same as for the proton running. The hadron arm inefficiency averaged $1.7 \%$ for the backward angle data and $2.5 \%$ for the forward angle measurements. A systematic error of $0.5 \%$ is applied to these results. These values are summarized in Table VI.

As mentioned previously, the trigger inefficiency comes from both inefficiences in the trigger logic and in the scintillators themselves. Although included in the above correction, inefficiences in the scintillators were studied by taking the ratio of the number of events that fired only the other scintillator (obtained from the so-called "junk" triggers - see Section 2.8) to the number of events that fired both scintillators. 
TABLE VI: Trigger inefficiency corrections.

\begin{tabular}{|c|c|c|c|c|}
\hline & Run Type & $\begin{array}{c}\text { HRSE Ineff. } \\
(\%)\end{array}$ & $\begin{array}{c}\text { HRSH Ineff. } \\
(\%)\end{array}$ & $\begin{array}{c}\text { Total } \\
(\%)\end{array}$ \\
\hline \hline Proton & Backward angle & 1.5 & 1.0 & $2.5 \pm 0.3$ \\
data & Forward angle & 1.0 & 1.8 & $2.8 \pm 0.3$ \\
\hline Deuteron & Backward angle & 1.5 & 1.7 & $3.2 \pm 0.5$ \\
data & Forward angle & 1.0 & 2.5 & $3.5 \pm 0.5$ \\
\hline
\end{tabular}

For the proton data, the trigger inefficiences for EARM S1, EARM S2 and HARM S1 were found to be approximately 0.3\%. HARM S2 exhibited an inefficiency of $1.0 \%$. The extra $0.7 \%$ was due to proton losses in HARM S1. For the deuteron data, the inefficiency of EARM S1, EARM S2 and HARM S1 should be the same. The inefficiency of HARM S2 coming from deuteron losses in HARM S1 should double from that of the proton data $(0.7 \% \rightarrow 1.4 \%)$, leading to a total of $1.7 \%$ inefficiency in HARM S2 for deuteron detection. No separate correction was made to the data to correct for scintillator inefficiencies, as it was already included in the trigger inefficiency correction.

The remaining trigger inefficiency came from inefficiencies in the trigger logic itself. Specifically, a small fraction of good events were classified as S2 or S4 "junk" triggers instead of S1 or S3 triggers as they should have been.

\section{Tracking Inefficiency}

The remaining detector inefficiency is a combination of the tracking inefficiencies of the drift chambers (on both spectrometers) and the inefficiencies of the Čerenkov and shower counter (on the electron arm only). For the vertical drift chambers, the tracking efficiency was defined as the ratio of the number of events for which at least one track was found to the number of good events that should have yielded a track. A good event was defined as one that satisfied the following conditions:

- The event had to have a good raw coincidence time in the range of real 
coincidence events (i.e. a $T C$ within the limits of the peak shown in Figure 40).

- The EP Ratio had to be between 0.50-2.50 for the backward angle data and 0.65-2.50 for the forward angle data.

- The Čerenkov ADC sum was required to be between channels 500-8000.

Figure 42 shows the tracking efficiencies of the electron arm (top) and hadron arm (bottom) VDC. As this figure shows, the VDCs were almost 100\% efficient, so no tracking inefficiency correction was applied to the data.

\section{Čerenkov Inefficiency}

The Cerenkov inefficiency was defined as the ratio of the number of events for which the ADC sum was not within the limits of channels 500-8000 to the number of good events where the sum should have been between these limits. A good event must satisfy the following conditions:

- It was a coincidence event within the real coincidence time peak.

- At least one track was reconstructed in the electron arm VDC.

- The EP Ratio was between 0.50-2.50 for the backward angle data and 0.652.50 for the forward angle data.

The inefficiency of the Cerenkov detector as a function of $E^{\prime}$ (the scattered electron energy), is shown in Figure 43. Most of this inefficiency was caused by events which yielded an ADC sum which was less than channel 500. A 0.4\% Čerenkov inefficiency correction was applied to the cross section, with a $0.1 \%$ systematic uncertainty to account for the spread in the measurements.

\section{Shower Counter Inefficiency}

The shower counter inefficiency was defined as the ratio of the number of events for which the EP ratio was not within the limits of $0.50-2.50$ for the backward 

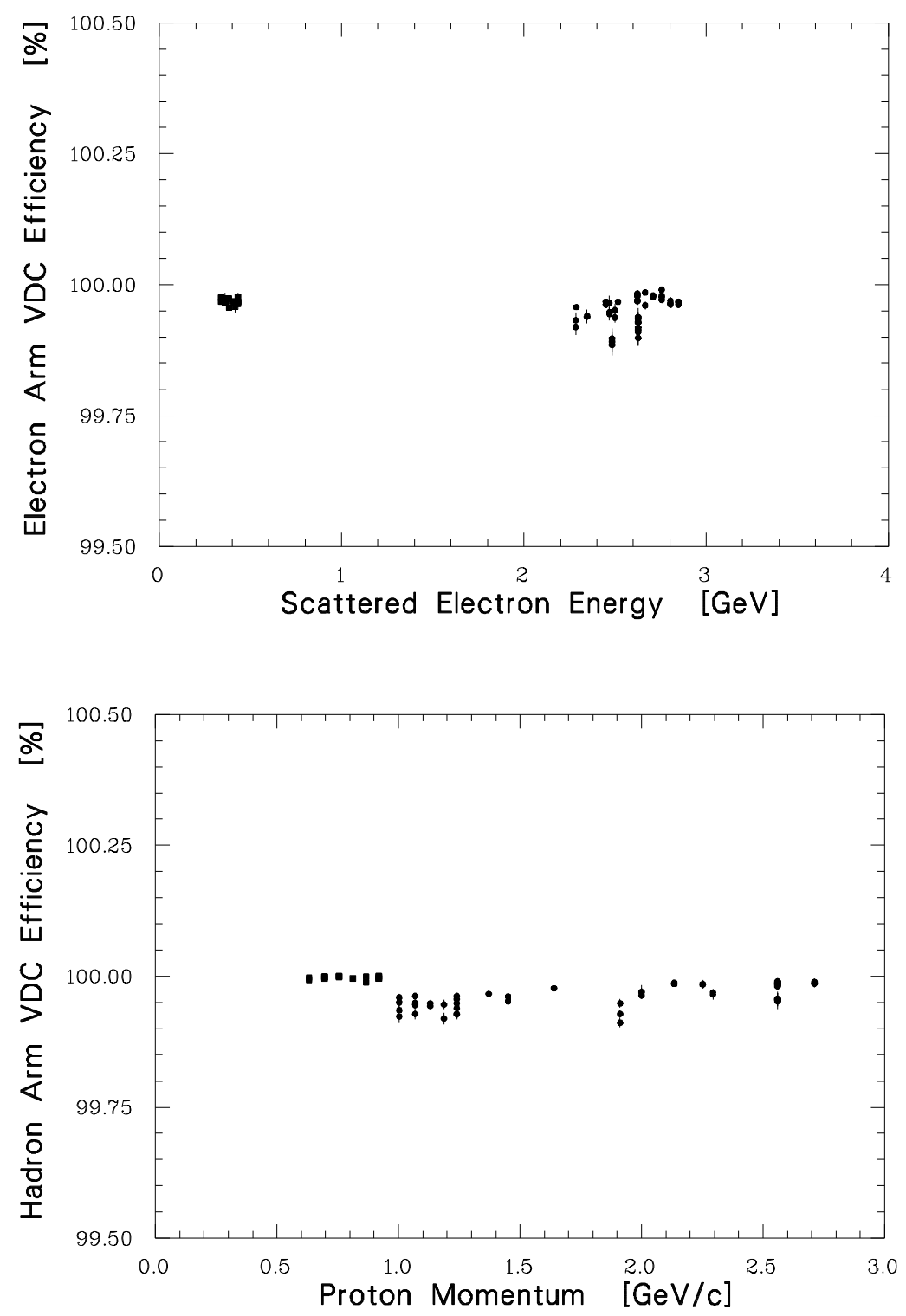

FIG. 42: Electron and Hadron ARM VDC efficiency. Top: Electron arm VDC efficiency versus the scattered electron energy. Bottom: Hadron arm VDC efficiency versus the scattered proton momentum. 


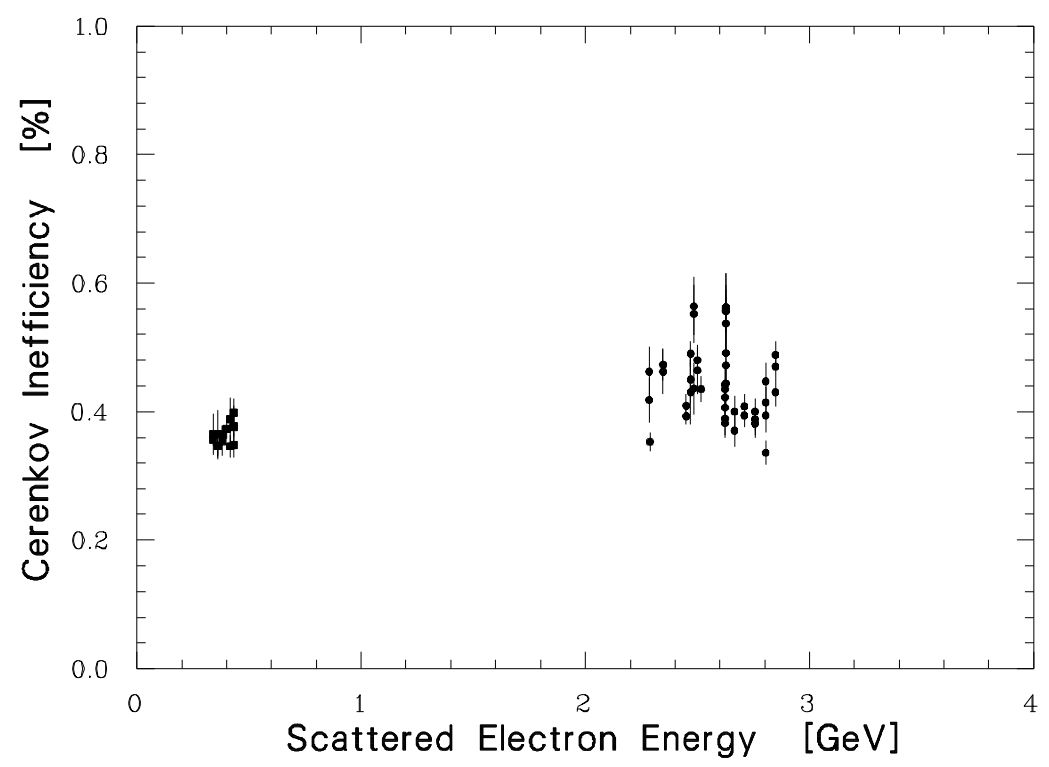

FIG. 43: Electron arm Čerenkov inefficiency versus the scattered electron energy.

angle data (within 0.65-2.50 for the forward angle data) to the number of good events where the ratio should have been within these limits. A good event must satisfy the following conditions:

- The event had to be a coincidence event within the real coincidence peak.

- At least one track was reconstructed in the electron arm VDC.

- The Čerenkov ADC sum was between channels 500-8000.

The inefficiency of the calorimeter was found to be a constant as a function of the scattered electron energy, $E^{\prime}$, for the backward angle (low energy) data, as seen in Figure 44. To correct for this inefficiency, a constant correction of $0.6 \%$ was applied to the cross section, with a $0.05 \%$ systematic uncertainty. For the forward angle (high energy) data, a constant correction of $0.95 \%$ was applied to the cross section, with of systematic uncertainty of $0.1 \%$. 


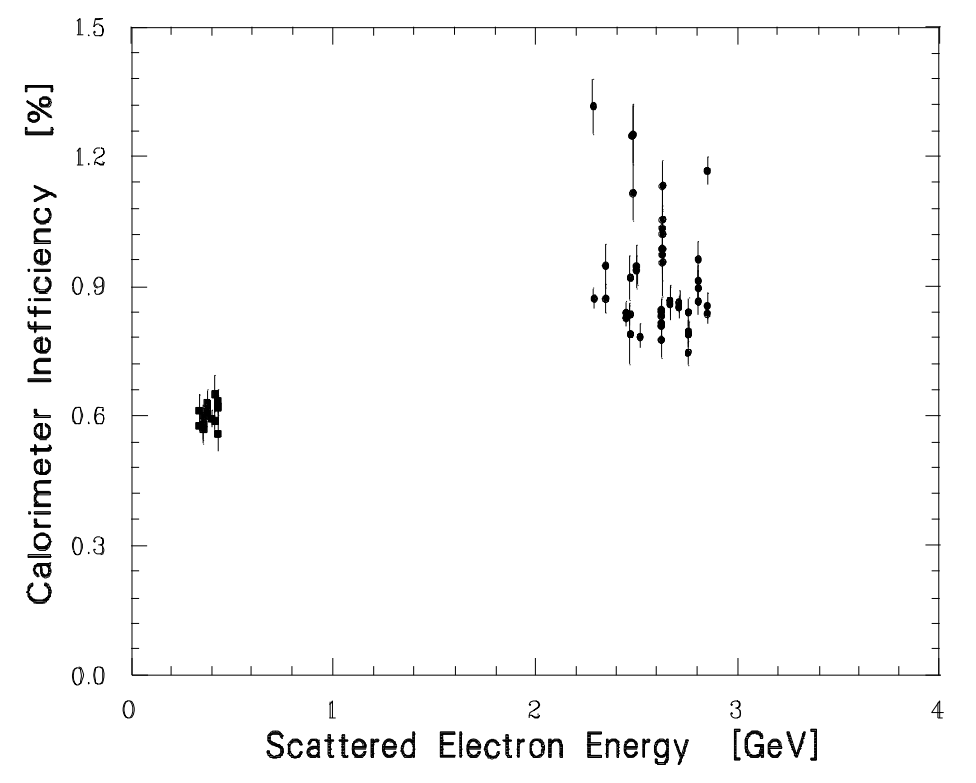

FIG. 44: Shower counter inefficiency for backward (low energy) and forward (high energy) angle data.

\subsubsection{Deadtime and Prescaling Correction}

A correction has to be made to the raw number of extracted coincidence events for the portion of events that are lost through computer deadtime and prescaling. Computer deadtime refers to events not being recorded because the data acquisition is busy processing a previous event (the data acquisition takes approximately $700 \mu \mathrm{s}$ to process an event). The prescaling correction accounts for the fraction of potentially good events that were artificially prescaled away in order to keep the computer deadtime reasonably low. This loss of events from both deadtime and prescaling can be corrected for by measuring the number of raw triggers versus the number of accepted triggers. The trigger setup for this experiment was presented in Table V of Section 2.8. The raw triggers are denoted by S1-S5, while the accepted triggers are denoted by T1-T5. The "livetime" for the coincidence trigger is simply the number of accepted triggers (T5) divided by the number of 
raw coincidence triggers (S5), so the deadtime correction is the inverse of this. The corrected number of coincidence enents is then given by

$$
N_{e d}^{c o r r}=\frac{S 5 \times N_{e d}^{r a w}}{T 5}
$$

where $N_{e d}^{r a w}$ is the number of raw (initial) coincidence events extracted from the analysis. For the elastic electron-deuteron data runs, the deadtime and prescaling correction is typically $10 \%$.

\subsubsection{Recoil Nuclear Interactions}

A portion of the recoiling nuclei from elastic electron scattering undergo further interaction along their way out of the target (both within the target fluid and within the aluminum walls of the target cell) or on their way through the scattering chamber and spectrometer windows. These interactions can cause the recoiling nucleus to be absorbed or deviated from its original path so that it fails to reach the recoil detectors. To account for these losses, a correction (denoted as $C_{r n i}$ ) is applied to the measured cross section. Note that this correction only takes into account recoil interactions in the target or spectrometer and scattering chamber windows. Recoil interactions within the detectors are taken into account with a detector inefficiency correction (Section 3.9.1).

For hydrogen, the $C_{r n i}$ correction is a combination of two corrections: one that takes into account proton-proton interactions within the target fluid and one that takes into account proton-aluminum interactions within the target cell walls, the aluminum scattering chamber exit window and the kapton (treated as $0.1 \mathrm{~mm}$ of aluminum) spectrometer entrance window. The percentage of protons lost in each material was calculated using the nuclear collision length, $\lambda$, of nucleons in matter:

$$
\lambda=\frac{A}{N_{a v} \rho \sigma},
$$

where $A$ and $\rho$ are, respectively, the atomic mass and density of the matter, $N_{a v}$ is Avogadro's number and $\sigma$ is the cross section of the reaction in question. 
The fraction of interacting particles is given by $P_{\text {int }}=1-\exp [-x / \lambda]$, where $x$ is the distance traveled through the material. For the $e-p$ calibration runs, the $C_{r n i}$ correction ranged from $0.7 \%-1.8 \%$, depending upon the momentum of the recoiling baryon.

For deuterium, the $C_{r n i}$ correction takes into account deuteron-deuteron interactions within the target fluid and deuteron-aluminum interactions within the target cell walls, the scattering chamber exit window and the spectrometer windows. The correction is done as above for hydrogen, using the nuclear collision length. The deuteron-deuteron cross section is approximated by $\sigma_{d d}=2\left(\sigma_{p p}+\sigma_{p n}\right)$, where $\sigma_{p p}$ and $\sigma_{p n}$ are the proton-proton and neutron-proton total cross sections. This leads to a $C_{r n i}$ correction ranging from $2.8 \%-5.1 \%$ for the elastic $e-d$ data.

\subsubsection{Target Density Correction}

Although the beam was rastered before it interacted with the target fluid, it was found that the density of the target varied as a function of the incident beam current. As described in Section 2.5.1, the target density change as a function of beam current was studied for both hydrogen and deuterium by monitoring the event rate as a function of current, from 10-120 $\mu \mathrm{A}$. Deuterium (hydrogen) was found to exhibit a $2.5 \%$ (5.0\%) density decrease at an incident beam current of 120 $\mu \mathrm{A}$ compared to its density at $10 \mu \mathrm{A}$. The density decrease was a linear function of current. In the cross section calculations, a corrected density was used instead of the nominal target liquid density, $\rho_{0}$, where this corrected density depended upon the average beam current, $I_{\text {ave }}$ (in $\mu \mathrm{A}$ ), during a run as described above:

$$
\rho=\rho_{0 d}\left(1-0.00021 \times I_{\text {ave }}\right),
$$

for deuterium and

$$
\rho=\rho_{0 h}\left(1-0.00042 \times I_{\text {ave }}\right) .
$$

for hydrogen. 


\subsection{Monte Carlo Simulation}

A Monte Carlo program [36] was used to model the double arm solid angle and focal plane event distributions for each of the elastic $e-p$ and $e-d$ kinematics of the experiment. The sequence of operations begins with the generation of an elastic electron-hadron scattering event in the target. The $z$ position of the event is generated using random numbers uniformly distributed over the target length $L$. The $x$ and $y$ coordinates of the interaction are generated taking into account the dimensions of the beam rastering. The electron scattering angles, $\theta_{e}$ and $\phi_{e}$, are generated using random numbers uniformly distributed between predefined $\theta$ and $\phi$ limits, which are chosen to be larger than the angular acceptance of the spectrometers. The incident electron's energy is modeled using a Gaussian distribution of random numbers around the central energy of the beam, with an energy spread of $0.01 \%$. The value of $E$ is corrected for ionization energy loss, multiple scattering and radiation loss for passage through a length $z$ of target material. To end the event generation sequence, the scattered electron energy, $E^{\prime}$, the recoil particle momentum, $P_{r}$, and the recoil scattering angles are calculated from elastic kinematics.

The Monte Carlo contains a realistic model of the two Hall A magnetic spectrometers. The particles can be transported through the spectrometers to the detectors in two ways. The first is by tracing each particle (ray) through a model of the spectrometer system. The second is by using TRANSPORT [37] matrix elements directly. TRANSPORT matrix elements are obtained by first ray-tracing a group of particles through the spectrometer. Then the matrix elements are obtained via a least squares fit to the initial and final coordinates of the particles. The matrix method uses less computer time than the ray-tracing method, but it requires an extensive study of the apertures of the system before it can be used.

For the ray-tracing method, information about each spectrometer element needs to be supplied. An element can be a magnetic element, such as a quadrupole or dipole, a drift length or an aperture. The information supplied for each magnet 
is its magnetic field, gradient and effective length. For the drifts, the drift distances are supplied, and for the apertures, their dimensions. Particles were traced through the three quadrupoles of the HRSE and HRSH using the ray-tracing method, in a series of 40 steps. At the end of each step, the coordinates of the particles with respect to the central ray were calculated using the Lorentz force equation $(\vec{F}=q \vec{v} \times \vec{B})$. For the EARM and HARM dipoles, a combination of the ray-tracing and the TRANSPORT matrix method was utilized. Particles were stepped through the dipole's uniform magnetic field in ten steps, using matrix elements to transfer the particles from one step to another. At the end of each step, particles were checked to see if they hit any apertures, which were assumed to be perfectly absorbing. This allowed for a clear picture of the acceptance-defining apertures, like the magnet pole faces or parts of the vacuum system, inside the spectrometers.

Electron and recoil distributions were plotted for events which successfully made it to the detectors. Figures 45 and 46 show a comparison between data and Monte Carlo predictions for the electron $y_{t r a}$ and $\phi_{t r a}$ distributions and the hadron $\theta_{\text {tra }}$ and $x_{\text {tra }}$ distributions at the wire chambers. The data are from a $\mathrm{H}\left(e, e^{\prime} p\right)$ run, utilizing the $15 \mathrm{~cm}$ hydrogen target, with an incident electron beam energy of $3.245 \mathrm{GeV}$. Both the electron and hadron distributions are described very well by the Monte Carlo, giving us confidence that the spectrometer and its apertures are well modeled.

\section{Solid Angle}

In this experiment, the scattered electron and recoiling deuteron are detected in coincidence, so the double-arm solid angle is a convolution of the acceptances of the two spectrometers coupled together by elastic kinematics and radiative effects. This double-arm solid angle is used in the cross section determination $\left(\Delta \Omega_{M C}\right.$ in Equation 36) and is determined from the Monte Carlo simulation used to model this experiment. It includes the effective elastic solid angle as well as the energy dependent portions of the radiative corrections. It is averaged over the target length $L$, the incident electron energy $E$ and the phase space of the incident 

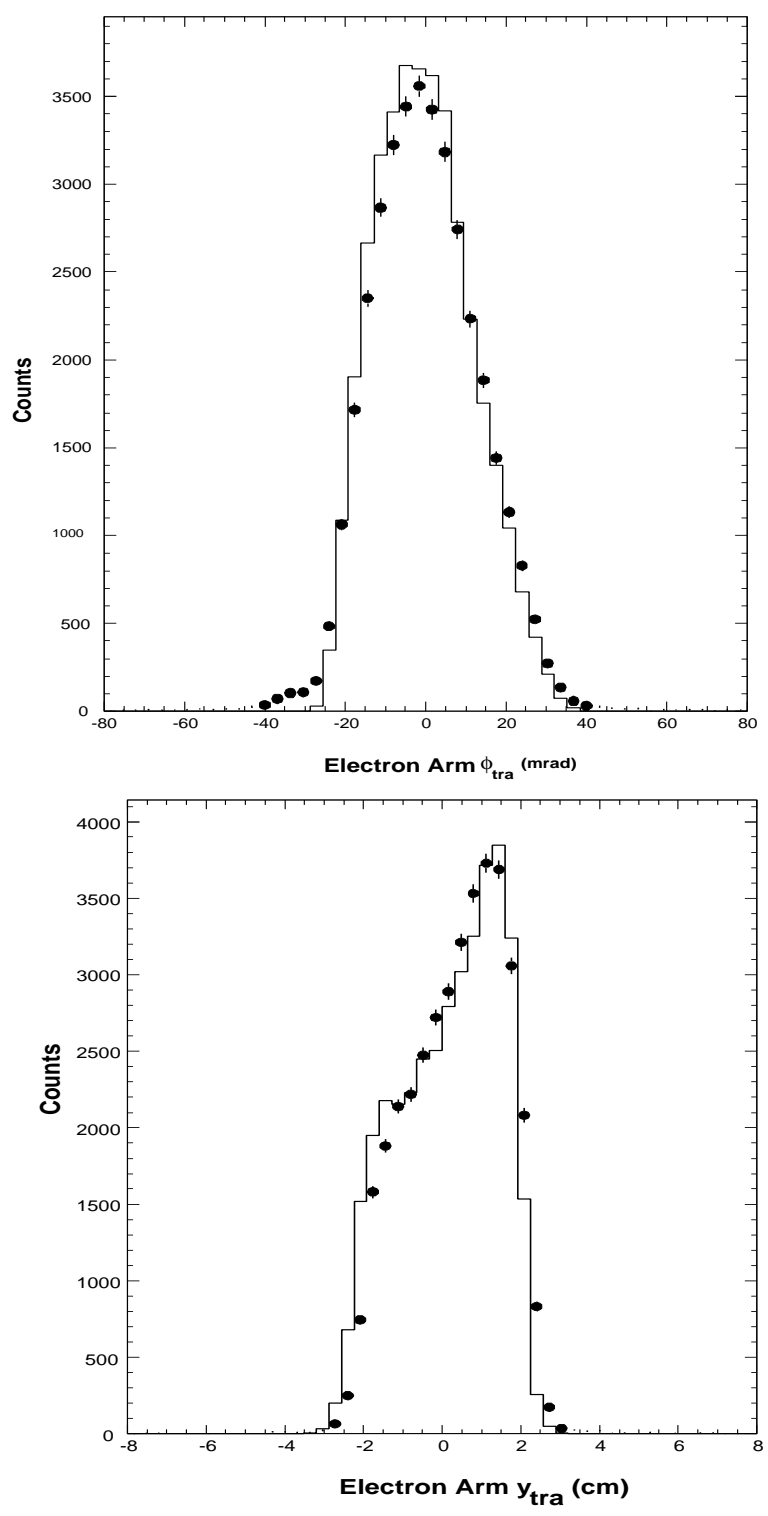

FIG. 45: Comparison of data to Monte Carlo for the electron spectrometer. The solid line is the Monte Carlo prediction and the circles are data from a $\mathrm{H}\left(e, e^{\prime} p\right)$ run. 

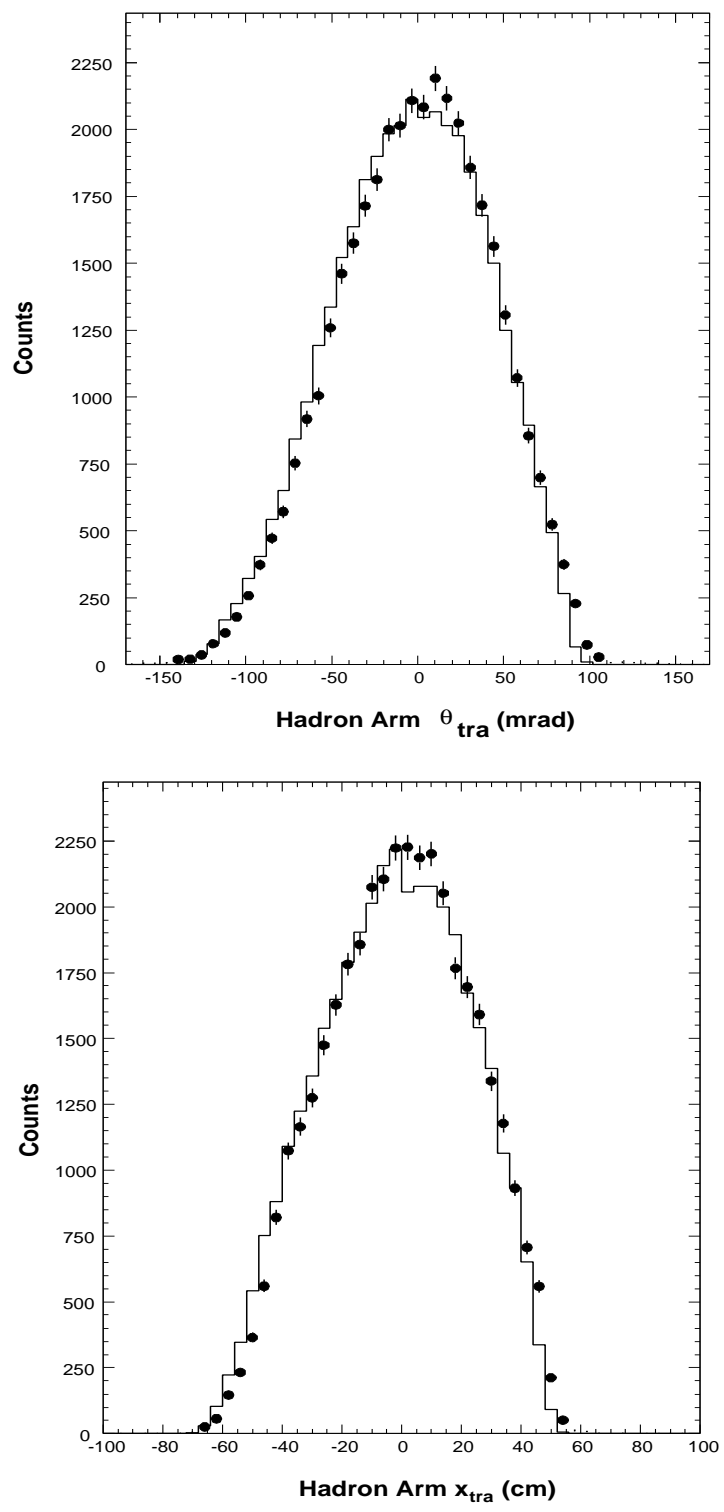

FIG. 46: Comparison of data to Monte Carlo for the hadron spectrometer. The solid line is the Monte Carlo prediction and the circles are data from a $\mathrm{H}\left(e, e^{\prime} p\right)$ run. 
beam. The computation of $\Delta \Omega_{M C}$ is described in detail in Reference [36] and summarized below.

The double-arm solid angle is given by the two dimensional integral

$$
\Delta \Omega_{M C}=\iint C_{M C} g(E, \Theta) f_{R C}\left(\Delta E^{\prime}, \Delta P_{r}\right) d \theta d \phi
$$

where $f_{R C}\left(\Delta E^{\prime}, \Delta P_{r}\right)$ represents the portion of the radiative corrections that depend upon the momentum acceptances, $\Delta E^{\prime}$ and $\Delta P_{r}$, of the electron and recoil spectrometers respectively. $\Delta \Omega_{M C}$ is weighted by

$$
g(E, \Theta)=\frac{\sigma(E, \Theta)}{\sigma\left(E_{0}, \Theta_{0}\right)}
$$

where $g(E, \Theta)$ represents the shape of the unradiated elastic cross section and is normalized to unity at $E_{0}, \Theta_{0} . C_{M C}$ represents the corrections due to ionization and multiple scattering [36]. The two dimensional integral of Equation (30) is performed using standard Monte Carlo techniques: for $N_{T}$ trial points randomly and uniformly distributed over the target length, between $\theta_{1} \leq \theta \leq \theta_{2}$ and $\phi_{1} \leq$ $\phi \leq \phi_{2}$, the integral is approximated by

$$
\int_{\theta_{1}}^{\theta_{2}} \int_{\phi_{1}}^{\phi_{2}} f(\theta, \phi) d \theta d \phi \approx\left(\theta_{2}-\theta_{1}\right)\left(\phi_{2}-\phi_{1}\right) \frac{1}{N_{T}} \sum_{i=1}^{N_{T}} f\left(\theta_{i}, \phi_{i}\right),
$$

where $f(\theta, \phi) \equiv C_{M C} g(E, \Theta) f_{R C}\left(\Delta E^{\prime}, \Delta P_{r}\right)$.

In the Monte Carlo loop, the energy loss due to ionization, multiple scattering and radiation are applied to each trial event. In the case of a good event, where both the scattered electron and recoiling nucleus pass all the way through the modeled spectrometers to the detectors, $f(\theta, \phi)=g(E, \Theta)$ otherwise $f(\theta, \phi)=0$. 


\subsection{Radiative Corrections}

Radiation effects necessitate important corrections in the analysis of electron scattering experiments. Radiative processes may be grouped into two categories: internal radiation and external radiation. Internal radiation is when the electron radiates real and virtual photons in the presence of the target nucleus involved in the elastic reaction (referred to as internal bremsstrahlung and vertex corrections, respectively). External radiation is when the electron radiates real and virtual photons in the presence of the Coulomb fields of nuclei other than the target nucleus (external bremsstrahlung).

The radiative corrections for this experiment are calculated within the Monte Carlo (Section 3.10) using the formalism of Reference [38]. Two factors account for the radiative corrections. The first, $\Delta \Omega_{M C}$, was discussed in Section 3.10. The second, $F\left(Q^{2}, T\right)$ accounts for the change in the interaction vertex due to radiative effects. It is given by

$$
\begin{aligned}
F\left(Q^{2}, T\right)=1+0.5772 b T & +\frac{2 \alpha}{\pi}\left[\frac{-14}{9}+\frac{13}{12} \ln \frac{Q^{2}}{m_{e}^{2}}\right] \\
& -\frac{\alpha}{2 \pi} \ln \frac{E}{E^{\prime}} \\
& +\frac{\alpha}{\pi}\left[\frac{1}{6} \pi^{2}-\Phi\left(\cos ^{2} \frac{\Theta}{2}\right)\right]
\end{aligned}
$$

where $\Phi$ is the Spence function [38] and $T$ is the total path length (in radiation lengths) that the electron traverses in the target before and after scattering. The factor $b$ is given by

$$
b=\frac{4}{3}\left[1+\frac{1}{9}\left[\frac{Z+1}{Z+\xi}\right]\left(\ln 183 Z^{-1 / 3}\right)^{-1}\right] .
$$

$Z$ is the atomic number of the material and $\xi$ is given by

$$
\xi=\frac{0.154 z_{c}^{2} Z t}{\beta^{2} A_{0}}
$$


where $z_{c}$ is the charge of the incident particle, $t$ is the path length in the material in $\mathrm{gm} / \mathrm{cm}^{2}, \beta=v / c$ and $A_{0}$ is the atomic mass of the material. $F\left(Q^{2}, T\right)$ is used in the cross section calculation as in Equation (36).

\subsection{Electron-Proton Elastic Cross Section Calibrations}

For each $A\left(Q^{2}\right)$ and $B\left(Q^{2}\right)$ kinematical point, a coincidence $e-p$ elastic cross section measurement was also made. The $e-p$ measurements calibrated our method of cross section determination, via comparisons of the measured $e-p$ cross section with a fit of the existing world data. The kinematics of the $e-p$ measurements were chosen so that the solid angle Jacobian matched that of the corresponding $e-d$ measurement. Data were taken both with and without the acceptance defining collimators in front of the spectrometers.

The elastic electron-proton cross sections were calculated using:

$$
\frac{d \sigma}{d \Omega}=\frac{N_{e p} C_{e f f}}{N_{i} N_{t}(\Delta \Omega)_{M C} F\left(Q^{2}, T\right)}
$$

where:

1. $\mathrm{N}_{e p}$ is the number of double-arm elastic e-p events extracted from the analysis.

2. $\mathrm{N}_{i}$ is the number of incident electrons.

3. $\mathrm{N}_{t}$ is the number of target nuclei (in units of nuclei $/ \mathrm{cm}^{2}$ ).

4. $(\Delta \Omega)_{M C}$ is the effective double-arm acceptance from a Monte Carlo simulation (Section 3.10).

5. $F\left(Q^{2}, T\right)$ is the portion of radiative corrections that depend only on $Q^{2}$ and $T$, where $T$ is the target length in radiation lengths. This factor is not 
included in the solid angle calculation, whereas radiative corrections that depend on the momentum acceptances of the spectrometers are.

6. $C_{\text {eff }}=C_{d e t} C_{c d t} C_{r n i}$ represents the corrections for detector and trigger inefficiencies $\left(C_{d e t}\right)$, computer deadtime and prescaling $\left(C_{c d t}\right)$ and losses of recoil nuclei due to nuclear interactions $\left(C_{r n i}\right)$. These corrections are discussed in detail in Section 3.9.

Tables VII, VIII and IX and Figure 47 show our measured $e-p$ cross sections (for the kinematics of the $A\left(Q^{2}\right)$ and $B\left(Q^{2}\right)$ measurements) divided by the Rosenbluth formula prediction using the form factors of Reference [39], which are a recent fit to the world proton form factor data:

$$
\begin{gathered}
G_{E}^{p}\left(Q^{2}\right)=\frac{1}{1+0.62 Q+0.68 Q^{2}+2.80 Q^{3}+0.83 Q^{4}}, \\
G_{M}^{p}\left(Q^{2}\right)=\frac{1}{1+0.35 Q+2.44 Q^{2}+0.50 Q^{3}+1.04 Q^{4}+0.34 Q^{5}},
\end{gathered}
$$

where $Q$ is in units of $\mathrm{GeV} / \mathrm{c}$. Using these form factors, the cross section for elastic electron-proton scattering is given by

$$
\frac{d \sigma}{d \Omega}=\sigma_{M}\left[\frac{G_{E}^{2}\left(Q^{2}\right)+\tau G_{M}^{2}\left(Q^{2}\right)}{1+\tau}+2 \tau G_{M}^{2}\left(Q^{2}\right) \tan ^{2}(\theta / 2)\right]
$$

where $\sigma_{M}$ and $\tau$ are as previously defined. The error in the parameterization is $\pm 3 \%$ for $Q^{2} \leq 10(\mathrm{GeV} / \mathrm{c})^{2}$, dominated by an overall normalization error in the data, increasing to $20 \%$ at $Q^{2}=30(\mathrm{GeV} / \mathrm{c})^{2}$, where the statistical accuracy dominates [39]. For the $A\left(Q^{2}\right)$ kinematics, the $e-p$ calibration data taken with the acceptance-defining collimators were found to agree with the cross section predicted by Equation (39) within $0.3 \%$ (on average). The $e-p$ calibration data taken without acceptance-defining collimators were found to be $2.6 \%$ higher, on 
TABLE VII: Ratio of the measured elastic $e-p$ cross sections to theory for the $A\left(Q^{2}\right)$ Kinematics (collimators IN). Listed are the measured elastic $e-p$ cross sections divided by the Rosenbluth prediction using the form factors of Reference [39]. This Table is for data taken with the acceptance defining collimators in front of both spectrometers. NOTE: For $A\left(Q^{2}\right)$ Kinematics.

\begin{tabular}{|c|c|c|c|}
\hline $\begin{array}{c}\text { Nominal } e-d Q^{2} \\
(\mathrm{GeV} / \mathrm{c})^{2}\end{array}$ & $\begin{array}{c}\text { Actual } e-p Q^{2} \\
(\mathrm{GeV} / \mathrm{c})^{2}\end{array}$ & $\begin{array}{c}\text { Cross Section Ratio } \\
\sigma / \sigma_{\text {model }}\end{array}$ & Error $_{\text {stat }+ \text { syst }}$ \\
\hline \hline 0.70 & 0.812 & 1.006 & \pm 0.02 \\
\hline 0.83 & 0.903 & 1.003 & \pm 0.02 \\
\hline 0.96 & 0.990 & 1.002 & \pm 0.02 \\
\hline 1.08 & 1.072 & 1.002 & \pm 0.02 \\
\hline 1.22 & 1.151 & 1.001 & \pm 0.02 \\
\hline 1.35 & 1.228 & 0.969 & \pm 0.02 \\
\hline 1.57 & 1.352 & 1.014 & \pm 0.02 \\
\hline 1.80 & 1.477 & 1.005 & \pm 0.02 \\
\hline 2.40 & 1.781 & 1.019 & \pm 0.02 \\
\hline 3.10 & 2.223 & 0.958 & \pm 0.02 \\
\hline 3.50 & 2.373 & 0.979 & \pm 0.02 \\
\hline 4.0 & 2.616 & 1.002 & \pm 0.02 \\
\hline 4.5 & 2.885 & 1.018 & \pm 0.02 \\
\hline 5.0 & 3.107 & 1.006 & \pm 0.02 \\
\hline 5.37 & 3.285 & 1.014 & \pm 0.02 \\
\hline 5.43 & 3.354 & 1.027 & \pm 0.02 \\
\hline 6.0 & 3.625 & 1.006 & \pm 0.02 \\
\hline
\end{tabular}


TABLE VIII: Ratio of the measured elastic $e-p$ cross sections to theory for the $A\left(Q^{2}\right)$ Kinematics (collimators OUT). Listed are the measured elastic e-p cross sections divided by the Rosenbluth prediction using the form factors of Reference [39]. This Table is for data taken without the acceptance defining collimators in front of both spectrometers. NOTE: For $A\left(Q^{2}\right)$ Kinematics.

\begin{tabular}{|c|c|c|c|}
\hline $\begin{array}{c}\text { Nominal } e-d Q^{2} \\
(\mathrm{GeV} / \mathrm{c})^{2}\end{array}$ & $\begin{array}{c}\text { Actual } e-p Q^{2} \\
(\mathrm{GeV} / \mathrm{c})^{2}\end{array}$ & $\begin{array}{c}\text { Cross Section Ratio } \\
\sigma / \sigma_{\text {model }}\end{array}$ & Error $_{\text {stat+syst }}$ \\
\hline \hline 0.70 & 0.811 & 1.043 & \pm 0.02 \\
\hline 0.83 & 0.902 & 1.055 & \pm 0.02 \\
\hline 0.96 & 0.988 & 1.048 & \pm 0.02 \\
\hline 1.08 & 1.069 & 1.036 & \pm 0.02 \\
\hline 1.22 & 1.148 & 1.045 & \pm 0.02 \\
\hline 1.35 & 1.222 & 1.026 & \pm 0.02 \\
\hline 1.80 & 1.476 & 1.006 & \pm 0.02 \\
\hline 3.10 & 2.214 & 0.994 & \pm 0.02 \\
\hline 3.50 & 2.364 & 1.011 & \pm 0.02 \\
\hline 4.0 & 2.663 & 1.017 & \pm 0.02 \\
\hline 4.5 & 2.872 & 1.020 & \pm 0.02 \\
\hline 5.0 & 3.102 & 1.044 & \pm 0.02 \\
\hline 5.37 & 3.284 & 1.045 & \pm 0.02 \\
\hline 5.43 & 3.341 & 1.025 & \pm 0.02 \\
\hline 6.0 & 3.611 & 1.011 & \pm 0.02 \\
\hline
\end{tabular}

TABLE IX: Ratio of measured elastic $e-p$ cross sections to theory for the $B\left(Q^{2}\right)$ kinematics.

\begin{tabular}{|c|c|c|c|}
\hline $\begin{array}{c}\text { Nominal } e-d Q^{2} \\
(\mathrm{GeV} / \mathrm{c})^{2}\end{array}$ & $\begin{array}{c}\text { Actual } e-p Q^{2} \\
(\mathrm{GeV} / \mathrm{c})^{2}\end{array}$ & $\begin{array}{c}\text { Cross Section Ratio } \\
\sigma / \sigma_{\text {model }}\end{array}$ & Error $_{\text {stat }+ \text { syst }}$ \\
\hline \hline 0.70 & 0.369 & 0.9651 & \pm 0.04 \\
\hline 0.83 & 0.437 & 0.9931 & \pm 0.04 \\
\hline 0.96 & 0.505 & 0.9804 & \pm 0.04 \\
\hline 1.08 & 0.572 & 1.003 & \pm 0.04 \\
\hline 1.22 & 0.639 & 1.017 & \pm 0.04 \\
\hline 1.35 & 0.707 & 0.9985 & \pm 0.04 \\
\hline
\end{tabular}


average, than the predicted values. Therefore, all elastic electron-deuteron cross section data (for the $A\left(Q^{2}\right)$ measurements) taken without the acceptance-defining collimators have been normalized downward by 2.6\% . For the $B\left(Q^{2}\right)$ kinematics, the $e-p$ calibration data were $0.8 \%$ lower, on average, than the world data prediction. No correction was applied to the $B\left(Q^{2}\right)$ data. 

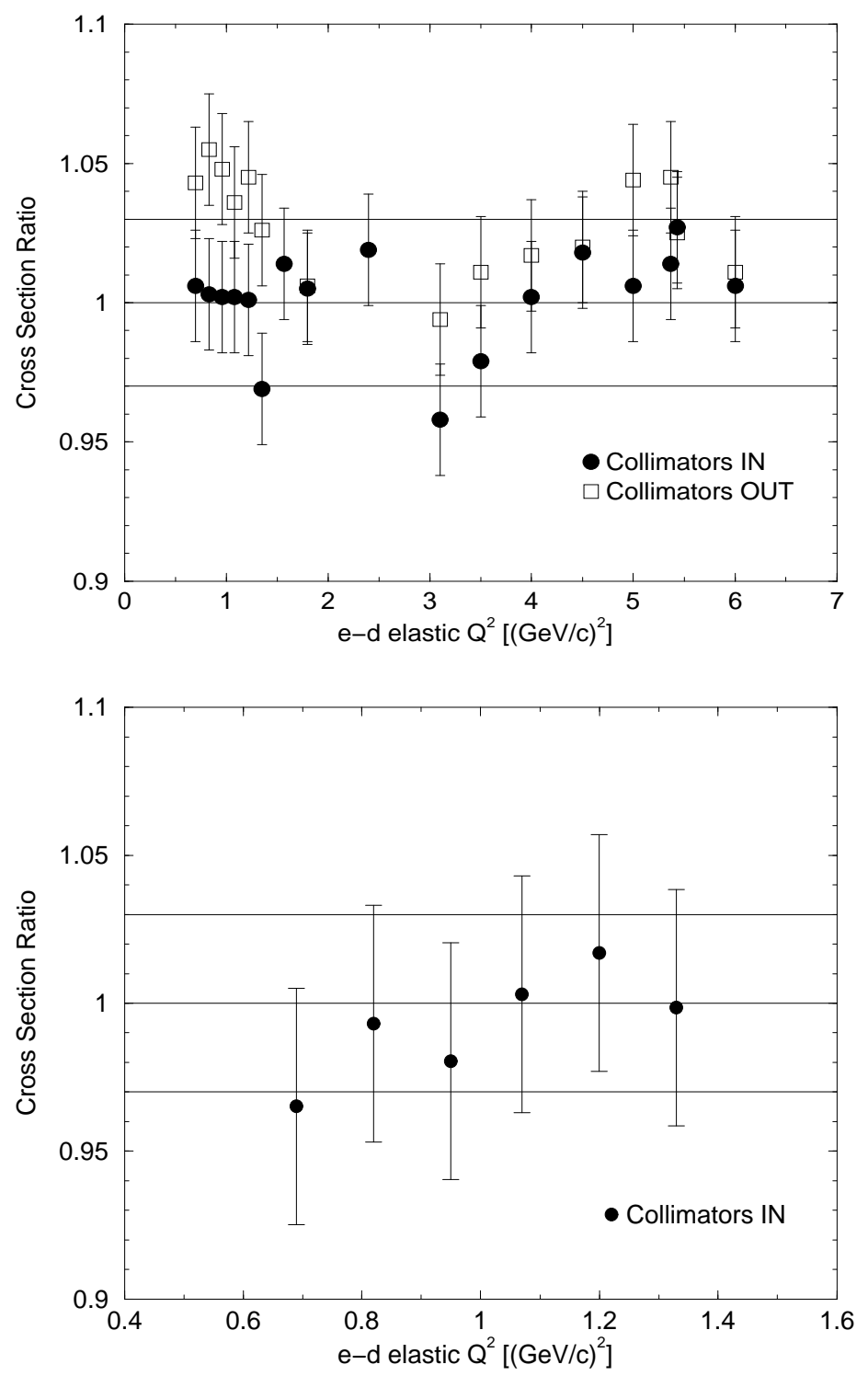

FIG. 47: Measured elastic $e-p$ cross sections divided by the Rosenbluth prediction using the form factors of Reference [39]. The solid lines at 1.03 and 0.97 represent the $\pm 3 \%$ error on the world data fit. The top plot is for the $A\left(Q^{2}\right)$ kinematics and the bottom plot for $B\left(Q^{2}\right)$. For the $B\left(Q^{2}\right)$ data, runs were taken only with the collimators in. 


\section{Chapter 4}

\section{RESULTS}

\section{1 $A\left(Q^{2}\right)$ Measurement}

The cross section for elastic electric-deuteron scattering was calculated using

$$
\frac{d \sigma}{d \Omega}=\frac{N_{e d} C_{e f f}}{N_{i} N_{t}(\Delta \Omega)_{M C} F\left(Q^{2}, T\right)},
$$

where $N_{e d}$ is the number of double-arm elastic $e$ - $d$ events extracted from the analysis and all other quantities are as in Equation (36). The measured cross sections for each of our kinematics are shown in Table X, along with their statistical and systematic errors added in quadrature. The statistical error ranged from $\pm 1 \%$ to $\pm 29 \%$ at $Q^{2}=5.954(\mathrm{GeV} / \mathrm{c})^{2}$. The cross section measurement at $Q^{2}=5.957$ $(\mathrm{GeV} / \mathrm{c})^{2}$ has $100 \%$ error because there was only one event for this kinematical point. The total systematic error has been estimated to be $\pm 5.9 \%$ and is dominated by the $\pm 3.6 \%$ uncertainty in the double-arm solid angle. A breakdown of the systematic errors as they stand at this time is shown in Table XI.

$A\left(Q^{2}\right)$ was extracted from the cross section using the Rosenbluth formula presented in Chapter 1:

$$
\frac{d \sigma}{d \Omega}=\sigma_{M}\left[A\left(Q^{2}\right)+B\left(Q^{2}\right) \tan ^{2}(\theta / 2)\right]
$$


TABLE X: Measured cross sections for the 18 kinematical points taken for the $A\left(Q^{2}\right)$ extraction. The error is the systematic and statistical error added in quadrature.

\begin{tabular}{|c|c|c|c|}
\hline$Q^{2}(\mathrm{GeV} / \mathrm{c})^{2}$ & $\theta_{e}$ (degrees) & Cross Section $\left(\mathrm{cm}^{2} / \mathrm{sr}\right)$ & Error $_{\text {stat }+ \text { syst }}\left(\mathrm{cm}^{2} / \mathrm{sr}\right)$ \\
\hline \hline 0.693 & 15.22 & $3.81 \times 10^{-34}$ & $\pm 0.23 \times 10^{-34}$ \\
\hline 0.821 & 16.67 & $1.56 \times 10^{-34}$ & $\pm 0.09 \times 10^{-34}$ \\
\hline 0.948 & 18.03 & $7.16 \times 10^{-35}$ & $\pm 0.43 \times 10^{-35}$ \\
\hline 1.076 & 19.32 & $3.60 \times 10^{-35}$ & $\pm 0.22 \times 10^{-35}$ \\
\hline 1.203 & 20.56 & $1.94 \times 10^{-35}$ & $\pm 0.12 \times 10^{-35}$ \\
\hline 1.331 & 21.77 & $9.89 \times 10^{-36}$ & $\pm 0.59 \times 10^{-36}$ \\
\hline 1.550 & 23.77 & $3.58 \times 10^{-36}$ & $\pm 0.22 \times 10^{-36}$ \\
\hline 1.780 & 25.79 & $1.39 \times 10^{-36}$ & $\pm 0.08 \times 10^{-36}$ \\
\hline 2.377 & 30.82 & $1.32 \times 10^{-37}$ & $\pm 0.08 \times 10^{-37}$ \\
\hline 3.040 & 27.98 & $2.33 \times 10^{-38}$ & $\pm 0.18 \times 10^{-38}$ \\
\hline 3.446 & 33.25 & $5.18 \times 10^{-39}$ & $\pm 0.45 \times 10^{-39}$ \\
\hline 3.956 & 36.59 & $1.29 \times 10^{-39}$ & $\pm 0.14 \times 10^{-39}$ \\
\hline 4.445 & 36.24 & $4.43 \times 10^{-40}$ & $\pm 0.68 \times 10^{-40}$ \\
\hline 4.951 & 39.30 & $1.17 \times 10^{-40}$ & $\pm 0.21 \times 10^{-40}$ \\
\hline 5.351 & 37.18 & $4.7 \times 10^{-41}$ & $\pm 1.4 \times 10^{-41}$ \\
\hline 5.357 & 41.83 & $4.5 \times 10^{-41}$ & $\pm 1.3 \times 10^{-41}$ \\
\hline 5.954 & 40.56 & $3.5 \times 10^{-41}$ & $\pm 1.0 \times 10^{-41}$ \\
\hline 5.957 & 45.74 & $8.1 \times 10^{-42}$ & $\pm 8.0 \times 10^{-42}$ \\
\hline
\end{tabular}


TABLE XI: Breakdown of $A\left(Q^{2}\right)$ systematic errors.

\begin{tabular}{|c|c|}
\hline Source & Magnitude \\
\hline beam charge & $2.0 \%$ \\
\hline beam energy & $1.5 \%$ \\
\hline target density & $1.0 \%$ \\
\hline electron angle & $1.5 \%$ \\
\hline double-arm solid angle & $3.6 \%$ \\
\hline Monte Carlo Statistics & $1.0 \%$ \\
\hline Electron detector inefficiencies (part of $\left.C_{\text {det }}\right)$ & $0.7 \%$ \\
\hline Hadron detector inefficiencies $\left(\right.$ part of $C_{\text {det }}$ ) & $0.7 \%$ \\
\hline recoil nuclear interactions $\left(C_{r n i}\right)$ & $2.6 \%$ \\
\hline VDC mistracking & $1.0 \%$ \\
\hline Accidental Subtraction & $1.5 \%$ \\
\hline \hline Total & $5.9 \%$ \\
\hline
\end{tabular}

where the Mott cross section, $\sigma_{M}=\frac{\alpha^{2} E^{\prime} \cos ^{2} \frac{\theta}{2}}{4 E^{3} \sin ^{4} \frac{\theta}{2}}$, is the cross section for scattering from a structureless target. $A\left(Q^{2}\right)$ was extracted from the measured cross sections shown in Table $\mathrm{X}$ assuming that $B\left(Q^{2}\right)$ does not contribute. This conclusion is supported by the existing $B\left(Q^{2}\right)$ data ([4], [10], [9]) which is shown in Figure 48 plotted on the same scale as the existing $A\left(Q^{2}\right)$ data ([3], [7], [9], [8]). From this Figure, it is seen that $B\left(Q^{2}\right)$ is always at least a factor of 10 lower than $A\left(Q^{2}\right)$. The contribution of $B\left(Q^{2}\right)$ to the measured cross section is further reduced by the $\tan ^{2}(\theta / 2)$ factor seen in the Rosenbluth formula (Equation (41)).

A fit to the existing $B\left(Q^{2}\right)$ data is shown in Figure 49. For each of the $A\left(Q^{2}\right)$ kinematics of this experiment, the magnitude of $B\left(Q^{2}\right)$ was extracted from the fit equation and the term $B\left(Q^{2}\right) \tan ^{2} \theta / 2$ was evaluated to calculate the magnetic structure function's contribution to the measured cross section. This contribution was found to be always less than $0.3 \%$, so it was neglected in the analysis and Equation (41) reduces to 


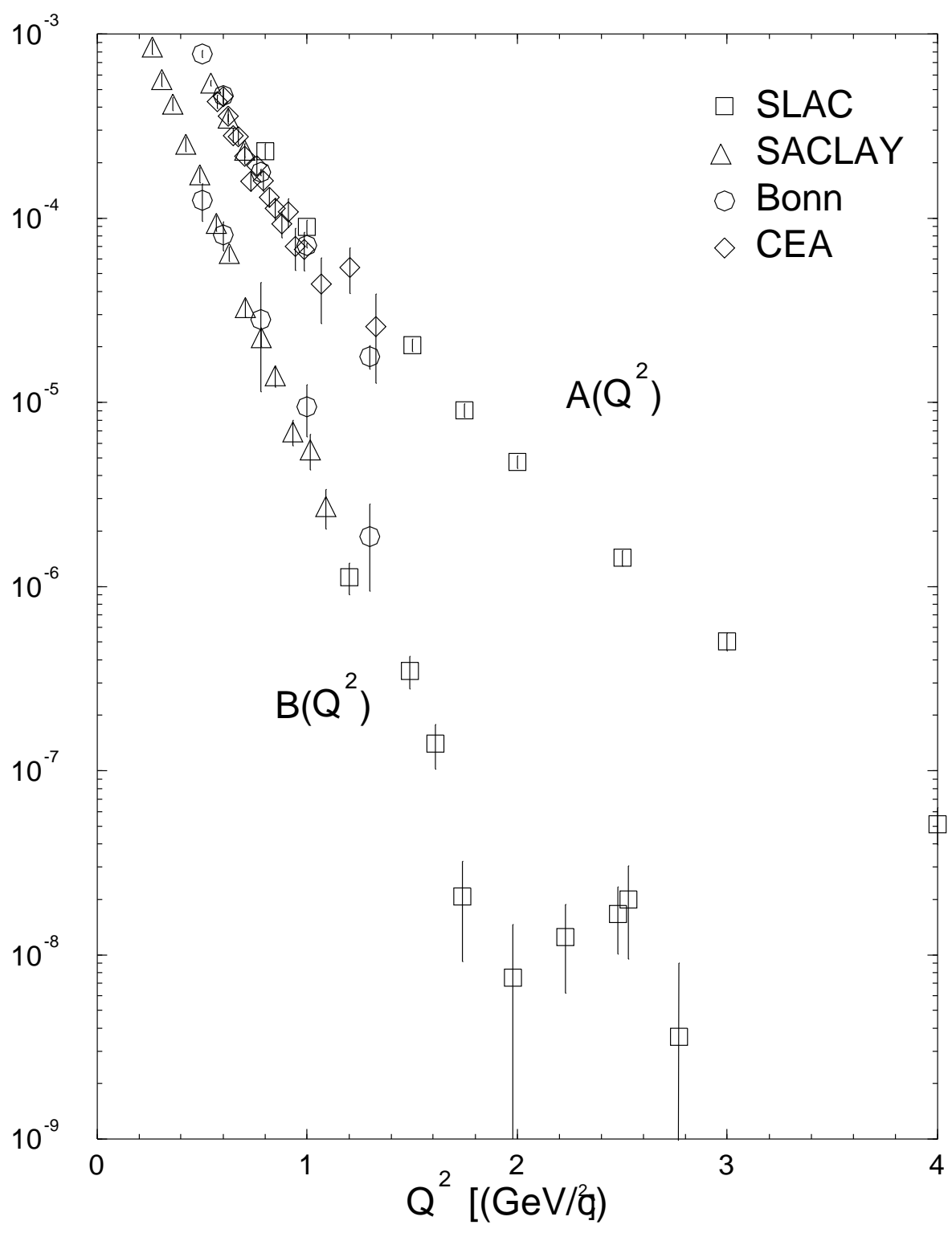

FIG. 48: Previous $A\left(Q^{2}\right)$ and $B\left(Q^{2}\right)$ data to $4(\mathrm{GeV} / \mathrm{c})^{2}$. The references for the data are in the text. 


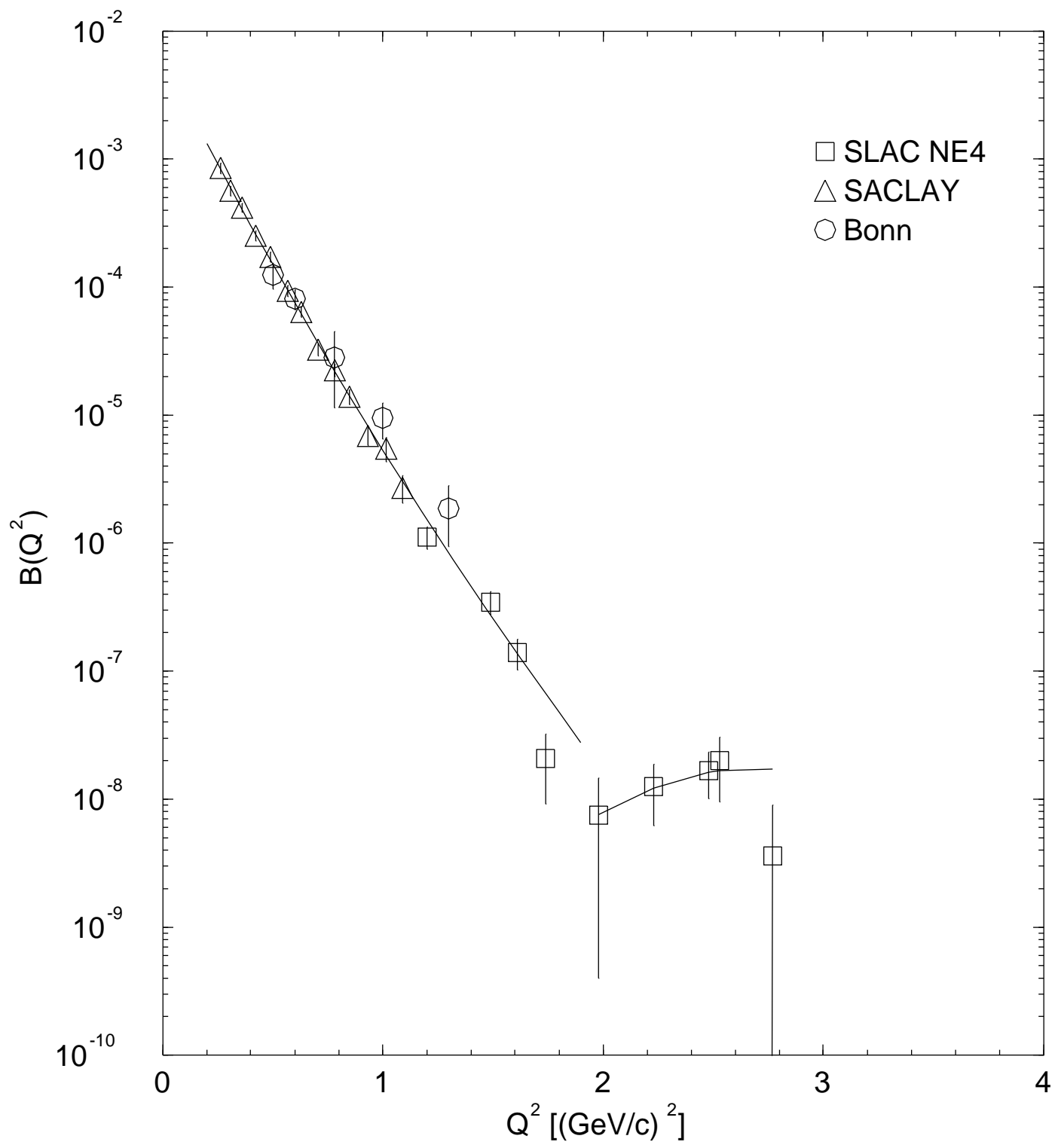

FIG. 49: Fit to the existing world $B\left(Q^{2}\right)$ data. The references are in the text. The data is fit in two parts: below and above the minimum. 


$$
\frac{d \sigma}{d \Omega}=\sigma_{M}\left[A\left(Q^{2}\right)\right] .
$$

The extracted values of $A\left(Q^{2}\right)$ are shown in Table XII. Each of the two highest $Q^{2}$ points represents the average of two cross section measurements (see Table X) taken with different beam energies $(4.0$ and $4.4 \mathrm{GeV})$. Figures 50 and 51 show the results of $A\left(Q^{2}\right)$ from this experiment [6] (solid circles), along with the previous data from SLAC [3], Saclay [7], Bonn [9] and CEA [8]. As seen in these figures, our results agree with the previous SLAC [3] and Saclay [7] measurements and are significantly higher than the Bonn [9] and CEA [8] data in their region of overlap, resolving the discrepancy that existed between the low $Q^{2}$ data sets. Our $A\left(Q^{2}\right)$ measurements continue the same smooth fall off to high momentum transfer (Figure 51), showing no evidence of any diffractive minima. Note that the three form factors that make up $A\left(Q^{2}\right)$ (Equation (6)) are predicted to exhibit a diffractive structure [40]. However, when they are combined with some kinematical factors to form $A\left(Q^{2}\right)$, this structure is smoothed out, producing the same smooth fall off of this structure function with increasing $Q^{2}$ that is observed. 
TABLE XII: Table of extracted $A\left(Q^{2}\right)$ values.

\begin{tabular}{|c|c|c|}
\hline$Q^{2}(\mathrm{GeV} / \mathrm{c})^{2}$ & $A\left(Q^{2}\right)$ & Error $_{\text {stat }+ \text { syst }}$ \\
\hline \hline 0.693 & $2.56 \times 10^{-4}$ & $\pm 0.15 \times 10^{-4}$ \\
\hline 0.821 & $1.52 \times 10^{-4}$ & $\pm 0.09 \times 10^{-4}$ \\
\hline 0.948 & $9.69 \times 10^{-5}$ & $\pm 0.58 \times 10^{-5}$ \\
\hline 1.076 & $6.52 \times 10^{-5}$ & $\pm 0.39 \times 10^{-5}$ \\
\hline 1.203 & $4.56 \times 10^{-5}$ & $\pm 0.27 \times 10^{-5}$ \\
\hline 1.331 & $2.96 \times 10^{-5}$ & $\pm 0.18 \times 10^{-5}$ \\
\hline 1.550 & $1.56 \times 10^{-5}$ & $\pm 0.10 \times 10^{-5}$ \\
\hline 1.780 & $8.62 \times 10^{-6}$ & $\pm 0.52 \times 10^{-6}$ \\
\hline 2.377 & $1.78 \times 10^{-6}$ & $\pm 0.11 \times 10^{-6}$ \\
\hline 3.040 & $3.32 \times 10^{-7}$ & $\pm 0.26 \times 10^{-7}$ \\
\hline 3.446 & $1.35 \times 10^{-7}$ & $\pm 0.12 \times 10^{-7}$ \\
\hline 3.956 & $5.23 \times 10^{-8}$ & $\pm 0.59 \times 10^{-8}$ \\
\hline 4.445 & $2.04 \times 10^{-8}$ & $\pm 0.31 \times 10^{-8}$ \\
\hline 4.951 & $7.9 \times 10^{-9}$ & $\pm 1.4 \times 10^{-9}$ \\
\hline 5.354 & $3.46 \times 10^{-9}$ & $\pm 0.75 \times 10^{-9}$ \\
\hline 5.955 & $2.85 \times 10^{-9}$ & $\pm 0.81 \times 10^{-9}$ \\
\hline
\end{tabular}




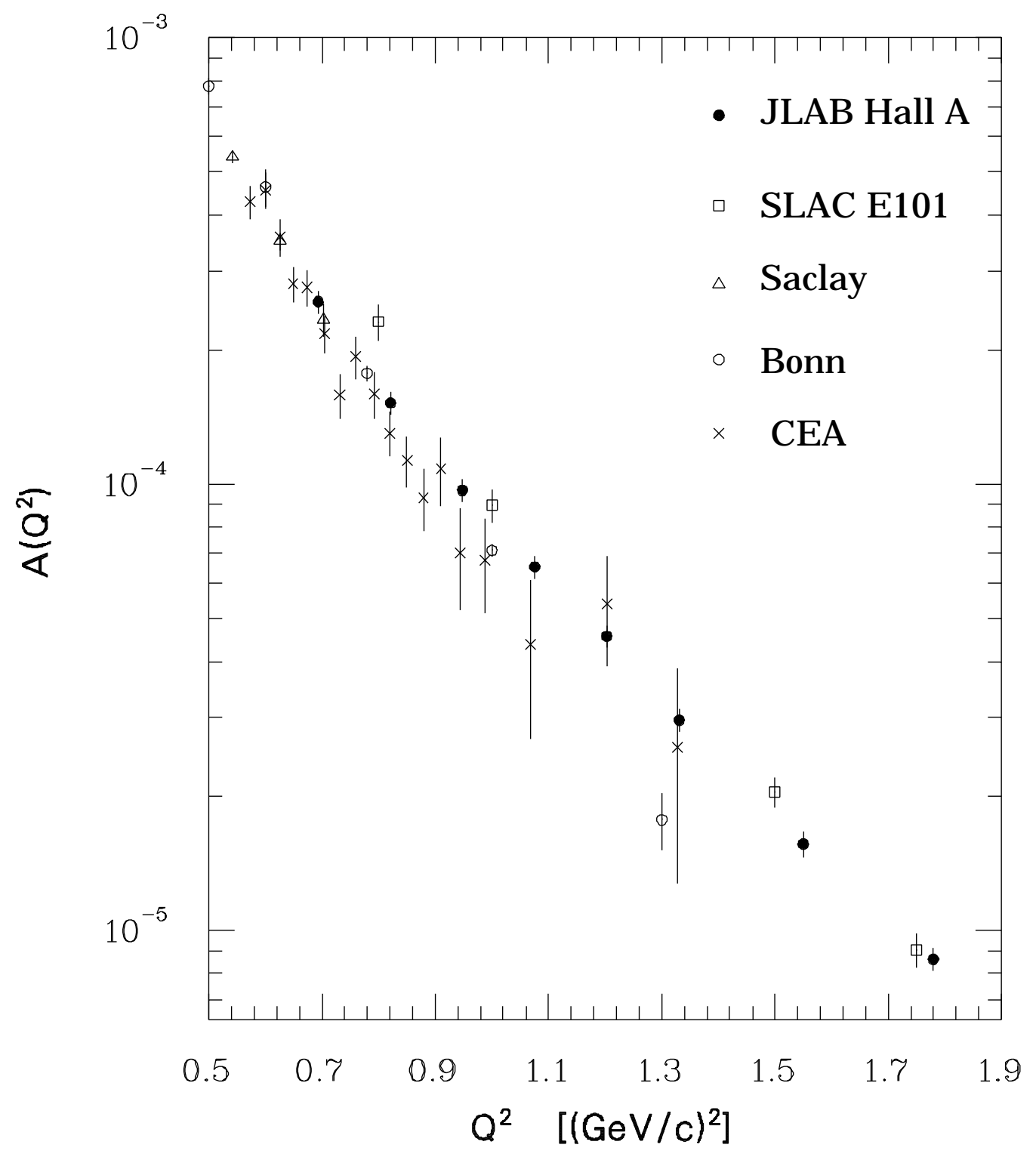

FIG. 50: Hall A $A\left(Q^{2}\right)$ measurements to $Q^{2}=1.9(\mathrm{GeV} / \mathrm{c})^{2}$. Also shown are previous measurements from SLAC [3], Saclay [7], Bonn [9] and CEA [8]. 


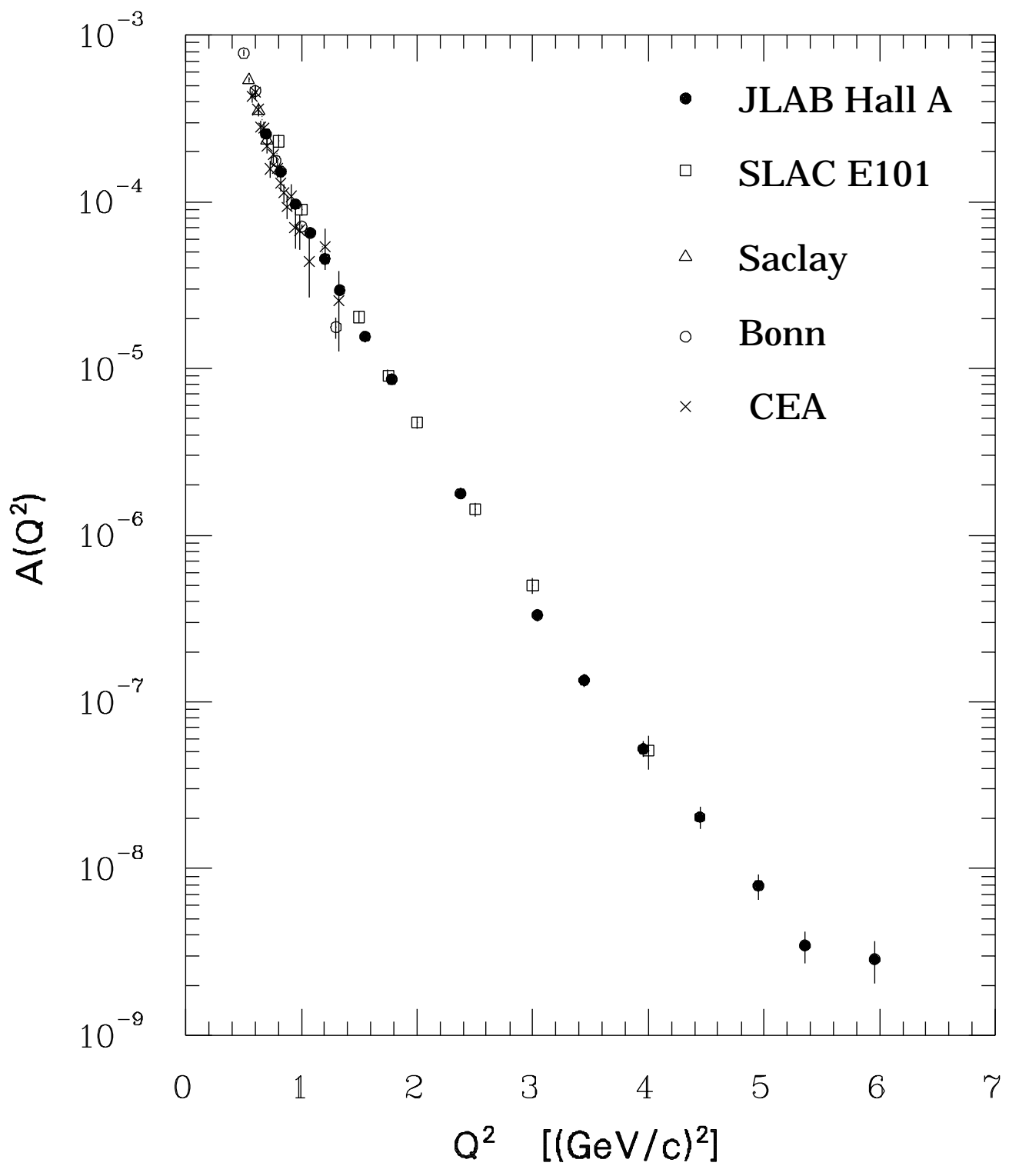

FIG. 51: Hall A $A\left(Q^{2}\right)$ measurements to $Q^{2}=6(\mathrm{GeV} / \mathrm{c})^{2}$. Also shown are previous measurements from SLAC [3], Saclay [7], Bonn [9] and CEA [8]. 


\section{2 $B\left(Q^{2}\right)$ Measurement}

The magnetic structure function, $B\left(Q^{2}\right)$, was extracted from the data using the Rosenbluth Separation technique. This technique involves measuring the elastic $e$ - $d$ cross section at two different angles (one forward, one backward) while keeping the momentum transfer fixed. At forward angles, the electric structure function's contribution to the cross section dominates, while at backward angles, the magnetic structure function's contribution to the cross section is enhanced because of the $\tan ^{2} \theta / 2$ factor in the Rosenbluth formula:

$$
\frac{d \sigma}{d \Omega}=\sigma_{M}\left[A\left(Q^{2}\right)+B\left(Q^{2}\right) \tan ^{2}(\theta / 2)\right]
$$

In the previous section, the measurement of the elastic forward angle cross section was described. An analogous procedure was followed to determine the cross section from the backward angle scattering data, where the electron spectrometer was positioned at its maximum backward angle of $144.5^{\circ}$. As before, the cross section for elastic $e$ - $d$ scattering is given by

$$
\frac{d \sigma}{d \Omega}=\frac{N_{e d} C_{e f f}}{N_{i} N_{t}(\Delta \Omega)_{M C} F\left(Q^{2}, T\right)},
$$

where $N_{e d}$ is the total number of coincidence events extracted from the analysis and all other quantities are as in the previous section. To do the Rosenbluth separation, the two cross sections measured at the same momentum transfer (one with a forward scattering angle, one with a backward angle) were combined to yield, in effect, two equations with two unknowns:

$$
\sigma_{F}=A\left(Q^{2}\right)+B\left(Q^{2}\right) \tan ^{2}\left(\theta_{F} / 2\right)
$$

and 
TABLE XIII: Table of extracted $B\left(Q^{2}\right)$ values.

\begin{tabular}{|c|c|c|}
\hline$Q^{2}(\mathrm{GeV} / \mathrm{c})^{2}$ & $B\left(Q^{2}\right)$ & Error $_{\text {stat }+ \text { syst }}$ \\
\hline \hline 0.691 & $3.27 \times 10^{-5}$ & $\pm 0.16 \times 10^{-5}$ \\
\hline 0.820 & $1.54 \times 10^{-5}$ & $\pm 0.06 \times 10^{-5}$ \\
\hline 0.949 & $7.24 \times 10^{-6}$ & $\pm 0.55 \times 10^{-6}$ \\
\hline 1.079 & $2.58 \times 10^{-6}$ & $\pm 0.28 \times 10^{-6}$ \\
\hline 1.208 & $1.44 \times 10^{-6}$ & $\pm 0.19 \times 10^{-6}$ \\
\hline 1.337 & $6.5 \times 10^{-7}$ & $\pm 1.2 \times 10^{-7}$ \\
\hline
\end{tabular}

$$
\sigma_{B}=A\left(Q^{2}\right)+B\left(Q^{2}\right) \tan ^{2}\left(\theta_{B} / 2\right),
$$

where $\sigma_{F}$ and $\sigma_{B}$ are respectively the forward and backward angle cross section measurements, taken at the same $Q^{2}$, normalized to their respective Mott cross section values.

Equations (45) and (46) were solved simultaneously at six different values of $Q^{2}$ to extract the magnetic structure function. Table XIII lists the extracted values of $B\left(Q^{2}\right)$. A close-up of these values is shown in Figure 52, plotted as solid circles, along with the existing data from SLAC [4], Saclay [10] and Bonn [9]. As seen, our magnetic structure function results agree very well with the previous results from all three other laboratories in their regions of overlap. The error bars on our results are much reduced over the previous measurements. Our data is shown plotted on an enlarged scale in Figure 53. We were able to make measurements only in the range of $0.7 \leq Q^{2} \leq 1.35(\mathrm{GeV} / \mathrm{c})^{2}$, so we fall short of measuring the magnetic structure function in the range of one of its measured minima, which is exhibited by the SLAC [4] data. Measurement of $B\left(Q^{2}\right)$ beyond $Q^{2} \approx 1.35(\mathrm{GeV} / \mathrm{c})^{2}$ requires the ablility to detect scattered electrons at very large scattering angles (close to $180^{\circ}$ ) where the magnetic structure function's contribution to the cross section is enhanced. 


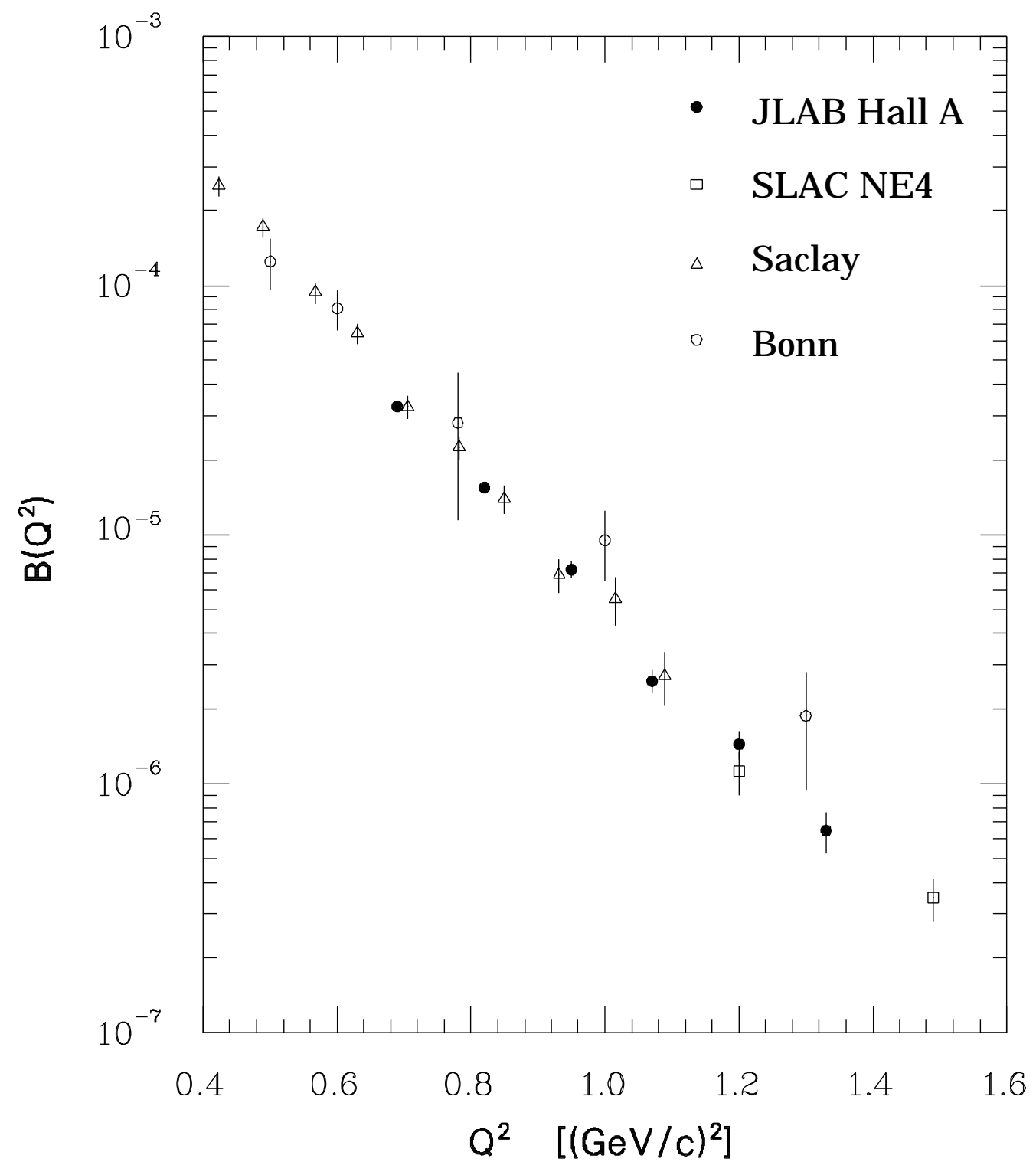

FIG. 52: Hall A $B\left(Q^{2}\right)$ measurements to $Q^{2}=1.6(\mathrm{GeV} / \mathrm{c})^{2}$. Also shown are previous measurements from SLAC [4], Saclay [10] and Bonn [9]. 


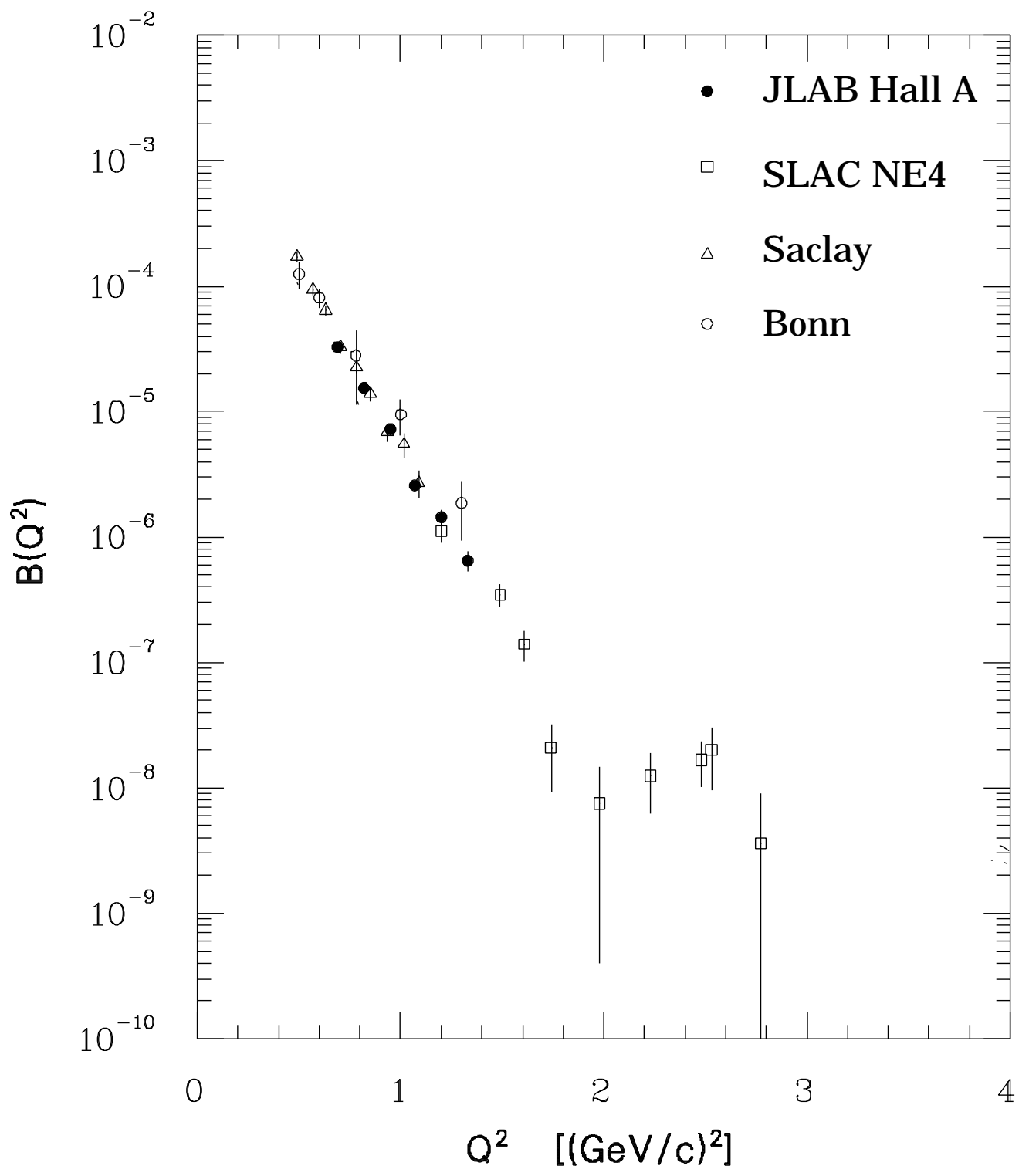

FIG. 53: $B\left(Q^{2}\right)$ measurements to $Q^{2}=4(\mathrm{GeV} / \mathrm{c})^{2}$. Also shown are previous measurements from SLAC [4], Saclay [10] and Bonn [9]. 


\section{Chapter 5}

\section{COMPARISON WITH THEORY}

The deuteron structure functions $A\left(Q^{2}\right)$ and $B\left(Q^{2}\right)$ can be calculated in terms of the nonrelativistic (NRIA) and relativistic impulse approximations (RIA), both with and without the inclusion of meson exchange currents (MEC). These approaches view the deuteron as a collection of interacting nucleons and mesons. At large momentum transfers, explicit quark and gluon degrees of freedom are expected to play an important role. At sufficiently large momentum transfer, the $Q^{2}$ evolution of the structure functions can be calculated in both quark dimensional scaling models and perturbative QCD (pQCD). In the sections that follow, I give a brief summary of the theoretical descriptions of the deuteron structure functions, as well as a comparison to the present and previous experimental data.

\subsection{Nonrelativistic Impulse Approximation}

In the impulse approximation, the virtual photon interacts with one of the nucleons in the deuteron and leaves the other as a spectator (Figure 54). As seen in Equations (6) and (7), the deuteron structure functions $A\left(Q^{2}\right)$ and $B\left(Q^{2}\right)$ are described in terms of three form factors, $F_{C}\left(Q^{2}\right), F_{Q}\left(Q^{2}\right)$ and $F_{M}\left(Q^{2}\right)$. The coupling of the virtual photon to the deuteron's charge yields a contribution to $F_{C}\left(Q^{2}\right)$, $F_{Q}\left(Q^{2}\right)$ and part of $F_{M}\left(Q^{2}\right)$ that is a product of the nucleons' electric form factors with an integral of the deuteron's D-State wave function, while its coupling 


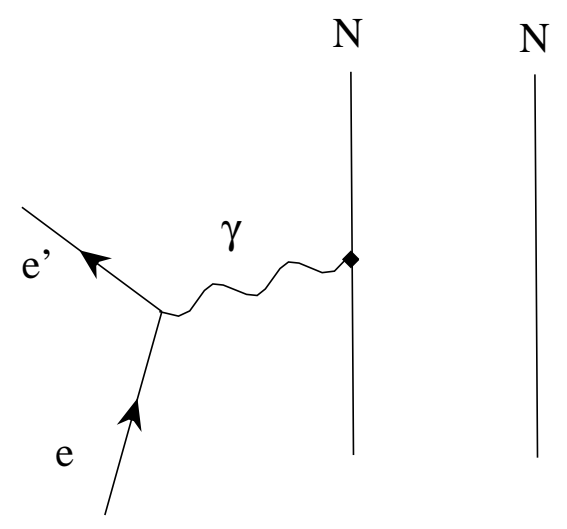

FIG. 54: Feynman diagram for the Impulse Approximation. Here, the virtual photon interacts with one of the nucleons. The blob at the photon-nucleon vertex represents the vertex form factor.

to the deuteron's spin adds a term to $F_{M}\left(Q^{2}\right)$ that is proportional to the nucleons' magnetic form factors. The expressions for the three form factors in terms of the nucleons' electromagnetic form factors and the deuteron wave function are given in Reference [41]:

$$
\begin{gathered}
F_{C}=\left(G_{E}^{p}+G_{E}^{n}\right) C_{E}, \\
F_{Q}=\left(G_{E}^{p}+G_{E}^{n}\right) C_{Q}, \\
F_{M}=\frac{M_{d}}{M_{p}}\left(\left(G_{M}^{p}+G_{M}^{n}\right) C_{S}+\frac{1}{2}\left(G_{E}^{p}+G_{E}^{n}\right) C_{L}\right),
\end{gathered}
$$

where $G_{E}^{p(n)}$ and $G_{M}^{p(n)}$ are the electric and magnetic form factors of the proton (neutron), respectively. These nucleon form factors take into account the fact that the proton and neutron are not point particles but have their own electromagnetic structure. $C_{E}, C_{Q}, C_{S}$ and $C_{L}$ give the distribution of the proton and neutron point currents inside the deuteron as determined by the deuteron wave function. They are given by: 


$$
\begin{gathered}
C_{E}=\int_{0}^{\infty}\left[u^{2}(r)+w^{2}(r)\right] j_{0}\left(\frac{Q r}{2}\right) d r \\
C_{Q}=\frac{3}{\sqrt{2} \tau} \int_{0}^{\infty} w(r)\left[u(r)-\frac{w(r)}{2 \sqrt{2}}\right] j_{2}\left(\frac{Q r}{2}\right) d r \\
C_{S}=\int_{0}^{\infty}\left\{\left[u^{2}(r)-\frac{1}{2} w^{2}(r)\right] j_{0}\left(\frac{Q r}{2}\right)+\frac{1}{2} w(r)\left[2^{1 / 2} u(r)+w(r)\right] j_{2}\left(\frac{Q r}{2}\right)\right\} \\
C_{L}=\frac{3}{2} \int_{0}^{\infty} w^{2}(r)\left[j_{0}\left(\frac{Q r}{2}\right)+j_{2}\left(\frac{Q r}{2}\right)\right] d r
\end{gathered}
$$

Here, $j_{0}\left(\frac{Q r}{2}\right)$ and $j_{2}\left(\frac{Q r}{2}\right)$ are spherical Bessel functions and $u(r)$ and $w(r)$ are the S- and D-state wave functions of the deuteron normalized so that $\int_{0}^{\infty}\left[u^{2}(r)+\right.$ $\left.w^{2}(r)\right] d r=1$.

There have been many NRIA calculations of the deuteron form factors, using a variety of deuteron wave functions and nucleon form factor parameterizations [1], [41]. The model of Wiringa, Stoks and Schiavilla [42], which employs the Argonne $v 18$ potential and the Höhler et al. [43] parameterization for the nucleon electromagnetic form factors, has been found to describe our $A\left(Q^{2}\right)$ data to 2.0 $(\mathrm{GeV} / \mathrm{c})^{2}$ when relativistic and meson exchange current corrections are included (see below).

The Argonne $v 18$ potential has a charge-independent part with 14 operators that is an updated version of the Argonne v14 potential, and a chargeindependence breaking part that has three charge-dependent and one chargeasymmetric operators. These charge-symmetry breaking terms arise because the potential has been fit to both $p p$ and $n p$ scattering data. As a further constraint, the potential has also been fit to low-energy $n n$ scattering parameters and the deuteron binding energy. In addition to the strong interaction, the potential includes terms to completely describe the electromagnetic interaction between the nucleons.

Table XIV shows a comparison between some static deuteron properties (values from Reference [1]), computed using the Argonne $v 18$ potential, and their experimental values. The deuteron binding energy, $E_{d}$, is fit exactly by construction. 
TABLE XIV: Deuteron static properties. The numbers are from Reference [1].

\begin{tabular}{|c|c|c|c|c|}
\hline & $\begin{array}{c}\text { Experimental } \\
\text { Value }\end{array}$ & $\begin{array}{c}\text { Prediction } \\
\text { Argonne } v 18\end{array}$ & $\begin{array}{c}\text { Prediction } \\
\text { Argonne } v 18+\mathrm{R}+\mathrm{MEC}\end{array}$ & Units \\
\hline$E_{d}$ & $2.224575(9)$ & 2.224575 & & $\mathrm{MeV}$ \\
\hline$\eta$ & $0.0256(4)$ & 0.0250 & & \\
\hline$r_{d}$ & $1.971(5)$ & 1.967 & & $\mathrm{fm}$ \\
\hline$\mu_{d}$ & $0.857406(1)$ & 0.847 & 0.871 & $\mu_{0}$ \\
\hline$Q_{d}$ & $0.2859(3)$ & 0.270 & 0.275 & $\mathrm{fm}^{2}$ \\
\hline$P_{d}$ & & 5.76 & & $\%$ \\
\hline
\end{tabular}

The predicted asymptotic $D / S$ state ratio, $\eta$, and deuteron radius, $r_{d}$, are both within 1.5 standard deviations of their experimental values. Both the deuteron magnetic and quadrupole moments, $\mu_{d}$ and $Q_{d}$, are underestimated, but when relativistic and meson exchange current contributions are added, the quadrupole moment becomes closer to the experimental value. The $S$ - and $D$-wave components of the deuteron wave function are shown in Figure 55, compared to those for the older Argonne $v 14$ model. It is seen that the short range behavior of the wave functions differs slightly between the two models.

The low $Q^{2} A\left(Q^{2}\right)$ data from this experiment [6] are shown in Figure 56 along with existing data from SLAC [3], Saclay [7], Bonn [9] and CEA [8]. The impulse approximation prediction (dotted line) underestimates the data. When relativistic corrections and meson exchange current contributions are included (solid line), the theoretical prediction agrees well with the data. The relativistic and meson exchange current corrections are included in the form of the electromagnetic current operator [42]. The electromagnetic current operator ([44], [45]) consists of oneand two-body parts. The one-body part has the standard NRIA form, with the inclusion of spin-orbit relativistic corrections. The two-body part contains contributions that correspond (in a One-Boson-Exchange picture) to those obtained from pion- and vector-meson ( $\rho$ and $\omega$ ) exchanges. The $\rho \pi \gamma$ MEC contribution is also taken into account. As seen in the Figure, relativistic and MEC corrections 


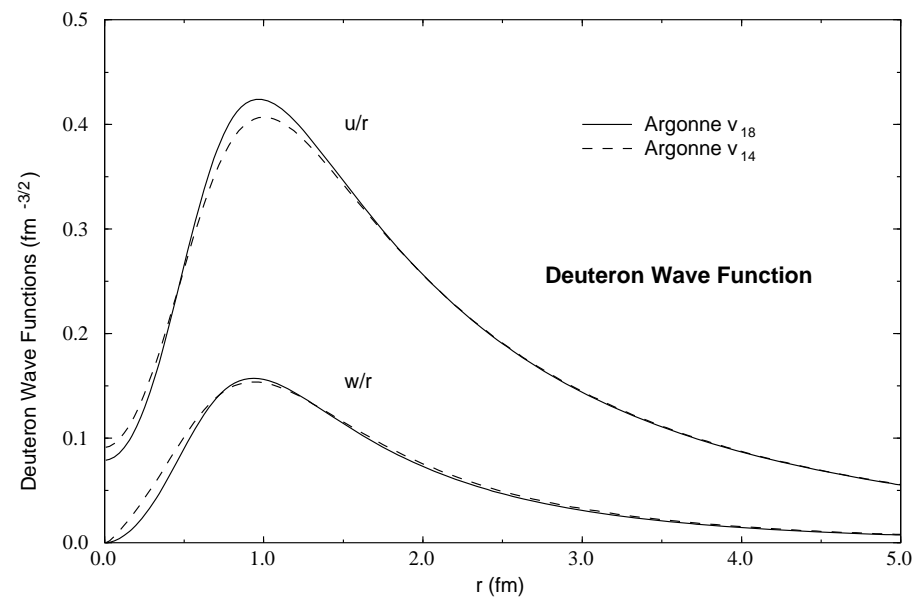

FIG. 55: Comparison of the deuteron wave function between two NRIA models. The deuteron $S$ - and $D$-state wave functions divided by $r$. The dashed curves are the predictions of the Argonne $v 14$ model, while the solid curves are of the Argonne $v 18$ model.

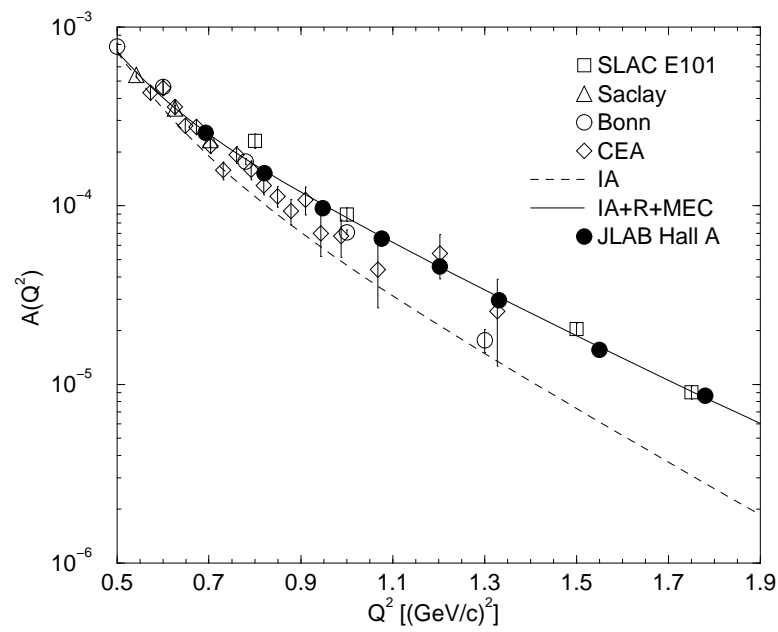

FIG. 56: NRIA comparison to $A\left(Q^{2}\right)$ data. The curves are from Reference [42]. The IA prediction (dashed line) underestimates the data. When relativistic corrections and meson exchange currents are added (solid line), the data are well predicted. 


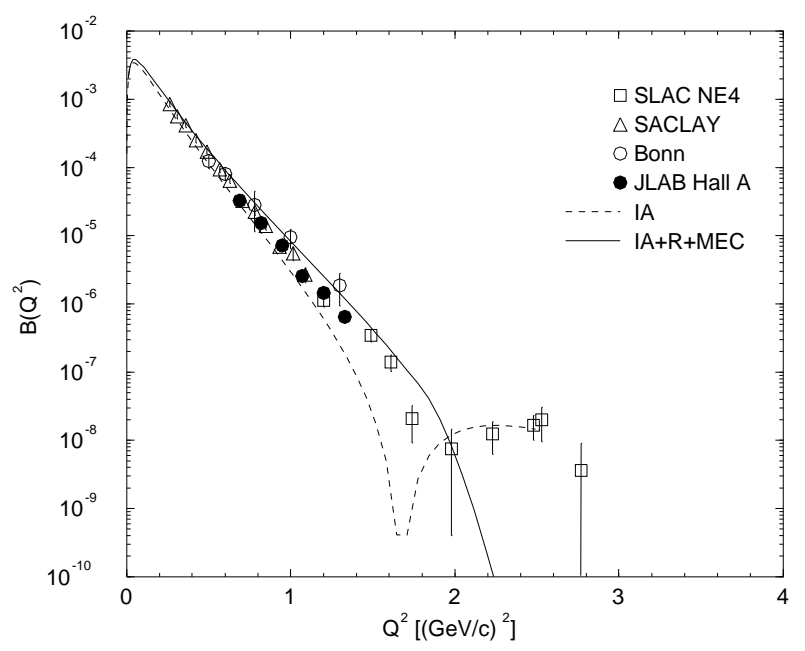

FIG. 57: NRIA comparison to $B\left(Q^{2}\right)$ data. The curves are the theoretical prediction of Reference [42].

play an important role in the context of this model.

Experimentally, the SLAC $B\left(Q^{2}\right)$ data [4] exhibit a minimum between $Q^{2}=$ 1.5-2.0 $(\mathrm{GeV} / \mathrm{c})^{2}$, corresponding to a sign change of the magnetic form factor. Our $B\left(Q^{2}\right)$ data in the range from $0.7 \leq Q^{2} \leq 1.35(\mathrm{GeV} / \mathrm{c})^{2}$ are shown in Figure 57 as solid circles. The NRIA predictions of Wiringa et al. [42] put the minimum close to $Q^{2}=1.7(\mathrm{GeV} / \mathrm{c})^{2}$ and underestimate our data. When relativistic and MEC corrections are included, the position of the minimum increases to approximately $2.2(\mathrm{GeV} / \mathrm{c})^{2}$. This results in an overestimate of our data. The leading two-body contributions to the magnetic structure function are from spin-orbit and quadratic spin-orbit interactions [42], which are of opposite sign. However, the overestimate of the data could indicate an insufficient degree of cancellation between them. The contribution from the $\rho \pi \gamma \mathrm{MEC}$ is small over the momentum transfer range considered here. 


\subsection{Relativistic Impulse Approximation}

When the momentum transferred to the target nucleus is larger than the nucleon mass, the usual nonrelativistic description of the nucleus is no longer reliable. It is therefore necessary to develop relativistically covariant models of nuclear systems.

In the one-photon exchange approximation, the invariant amplitude for elastic electron-deuteron scattering is a function of the electron and deuteron current matrix elements:

$$
\mathcal{M}=<k^{\prime} \lambda^{\prime}\left|j_{\mu}^{e l}\right| k \lambda>\frac{1}{q^{2}}<P^{\prime} M^{\prime}\left|J_{D}^{\mu}\right| P, M>.
$$

The electron current is $\left\langle k^{\prime} \lambda^{\prime}\left|j_{\mu}^{e l}\right| k \lambda\right\rangle=i e \bar{u}_{\lambda^{\prime}}\left(k^{\prime}\right) \gamma_{\mu} u_{\lambda}(k)$, where $k$ and $k^{\prime}$ are the initial and final electron momenta and $\lambda$ and $\lambda^{\prime}$ are the corresponding helicities. The deuteron current can be written in terms of three form factors, $\mathcal{F}_{1}, \mathcal{F}_{2}$ and $\mathcal{G}_{1}$, which are related to the usual charge, quadrupole and magnetic form factors of the deuteron:

$$
<P^{\prime} M^{\prime}\left|J_{\mu}^{D}\right| P, M>=e_{\rho}^{*}\left(P^{\prime}, M^{\prime}\right) J_{\mu}^{\rho \sigma} e_{\sigma}(P, M),
$$

with

$$
J_{\mu}^{\rho \sigma}=\left(P_{\mu}^{\prime}+P_{\mu}\right)\left[g^{\rho \sigma} \mathcal{F}_{1}\left(q^{2}\right)-\frac{q^{\rho} q^{\sigma}}{2 M_{d}^{2}} \mathcal{F}_{2}\left(q^{2}\right)\right]+I_{\mu \nu}^{\rho \sigma} q^{\nu} \mathcal{G}_{1}\left(q^{2}\right) .
$$

In Equations (55) and (56), $e_{\sigma}(P, M)$ is the deuteron polarization vector with helicity $M, P$ and $P^{\prime}$ are the initial and final deuteron momenta and $I_{\mu \nu}^{\rho \sigma}$ is the infinitesimal generator of the Lorentz transformations [56]. In terms of $\mathcal{F}_{1}, \mathcal{F}_{2}$ and $\mathcal{G}_{1}$, the charge, quadrupole and magnetic form factors are

$$
\begin{aligned}
F_{C} & =\mathcal{F}_{1}+(2 / 3) \tau\left(\mathcal{F}_{1}+(1+\tau) \mathcal{F}_{2}+\mathcal{G}_{1}\right) \\
F_{Q} & =\mathcal{F}_{1}+(1+\tau) \mathcal{F}_{2}+\mathcal{G}_{1}, \\
F_{M} & =\mathcal{G}_{1}
\end{aligned}
$$


where $\tau=Q^{2} / 4 M_{d}^{2}$ as previously defined. To evaluate the deuteron current matrix element, the deuteron electromagnetic current and wave function must first be calculated from a relativistic model.

As described in Reference [46], there are many relativistic methods available to perform a calculation of the deuteron current matrix element. The different approaches are outlined in Figure 58. The first (and most profound) choice is between "Hamiltonian dynamics" and "manifestly covariant dynamics". Hamiltonian dynamics [47] uses conventional quantum mechanics where the theoretical framework is well known and understood. Negative energy states are excluded. The disadvantage of this approach is that some of the Lorentz transformations include the interaction, and therefore cannot be evaluated without doing additional dynamical calculations. Manifestly covariant dynamics uses the Bethe-Salpeter formalism [48] and related quasipotential equations [49, 50], or the Equal Time Approximation. In this formalism, the Lorentz transformations are kinematic, i.e. they depend only on the spin and momenta of the particles and not on the interactions. Negative energy states are included in the dynamics and the theory is no longer a conventional quantum mechanics. This method is described in detail below.

Manifestly covariant dynamics for bound states can be said to have started with the introduction of the Bethe-Salpeter (BS) equation [48] in 1951. The scattering amplitude $M$ is given by the BS equation as

$$
M=V+V G_{B S} M,
$$

where $V$ is the relativistic kernel (potential) and $G_{B S}$ is the free two-body propagator which describes the propagation of two off-shell nucleons. Equation (58) is an iterative equation, which is solved repeatedly until the scattering amplitude converges.

The Bethe-Salpeter equation is a four-dimensional equation and therefore difficult to solve. This led to the development of so-called "quasipotential" approximations to the BS equation. In these equations, the propagator $G_{B S}$ is replaced 


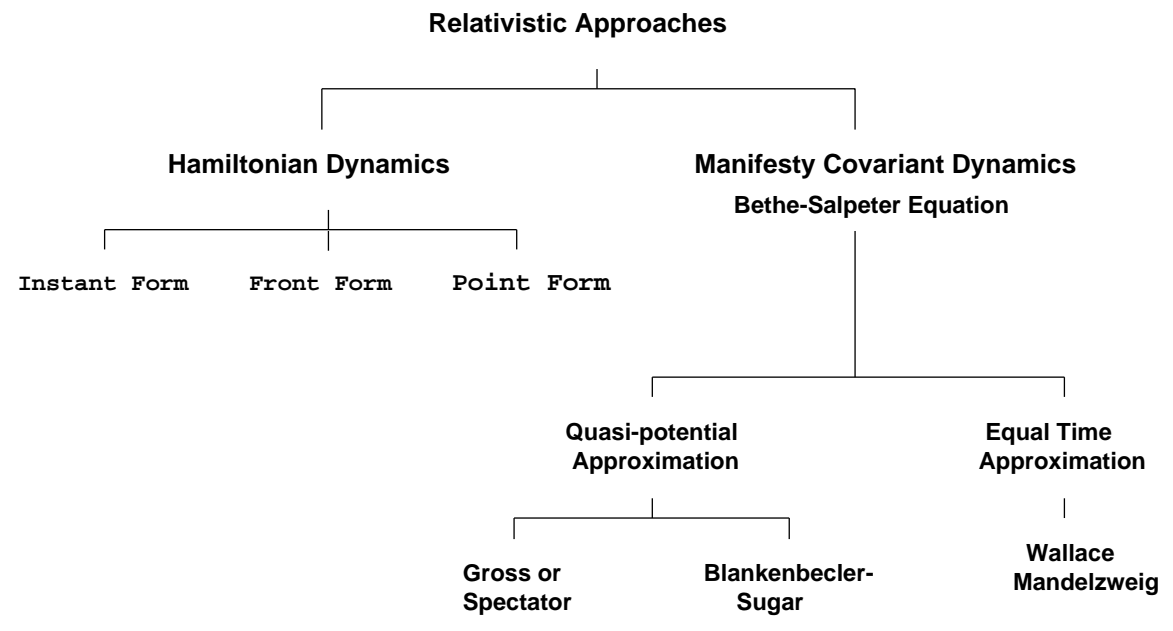

FIG. 58: Schematic of relativistic approaches.

by a new propagator $g$. The scattering amplitude is then written as

$$
M=U-U g M,
$$

where $U$ is the quasipotential defined as

$$
U=V-V\left(G_{B S}-g\right) U .
$$

The new propagator $g$ is chosen to include a one-dimensional delta function that constrains the relative energy of the propagating pair, reducing Equation (59) to a three dimensional equation. The quasipotential $U$ should be determined using Equation (60), but because of difficulties in solving this equation, $U$ is chosen to be the One-Boson-Exchange (OBE) potential, i.e. $U=V_{O B E}$, with meson-nucleon coupling constants chosen by fitting the $N N$ scattering data and the deuteron static properties. 
Two relativistic calculations of the deuteron structure functions which utilized quasipotential approximations to the Bethe-Salpeter equation have been compared to our data. The first is that of Van Orden, Divine and Gross [51], which employed the Gross or Spectator equation [49]. This equation is obtained by constraining the relative energy of the intermediate states by restricting one of the nucleons to its positive energy mass shell. For their calculation, a one-boson-exchange interaction kernel was employed. The meson-nucleon couplings used include offshell couplings and form factors which depend on the invariant masses of the three virtual particles connected at the interaction vertex.

The application of the Gross equation to the calculation of nucleon-nucleon scattering was described in Reference [52]. Four models of the $N N$ interaction, each with different one-boson-exchange kernels, were discussed. Each model was fitted to the NN phase shift data and constrained to fit the deuteron binding energy. The model compared to our data is a variation on model IIB and its parameters have been adjusted to fit the Nijmegen energy dependent phase shifts. This model used a one-boson-exchange kernel containing six mesons: $\pi, \eta, \sigma, \delta$, $\omega$ and $\rho$.

The wave functions for this model (IIB, solid lines) and a similiar model (W16, dashed lines) are shown in Figure 59. As seen in this Figure, the wave functions for relativistic models have four components: the usual $S$ and $D$ waves that appear in the nonrelativistic description of the deuteron and two $P$ waves (a spin triplet and singlet) of relativistic origin. These $P$ states arise because of the inclusion of negative energy intermediate states. The wave function is normalized as

$$
\int_{0}^{\infty} d r\left[u^{2}+w^{2}+v_{t}^{2}+v_{s}^{2}\right]+<\frac{\delta V}{\delta M_{d}}>=1
$$

where $u$ and $w$ represent the $S$ and $D$ states and $v_{t}$ and $v_{s}$ are the triplet and singlet states, respectively. The contributions to the normalization of the wave function from these components are $92.979 \%$ for the $S$ wave, $5.015 \%$ for the $D$ wave, $0.049 \%$ for the triplet $P$ wave and $0.009 \%$ for the singlet $P$ wave. The remaining $2 \%$ is 

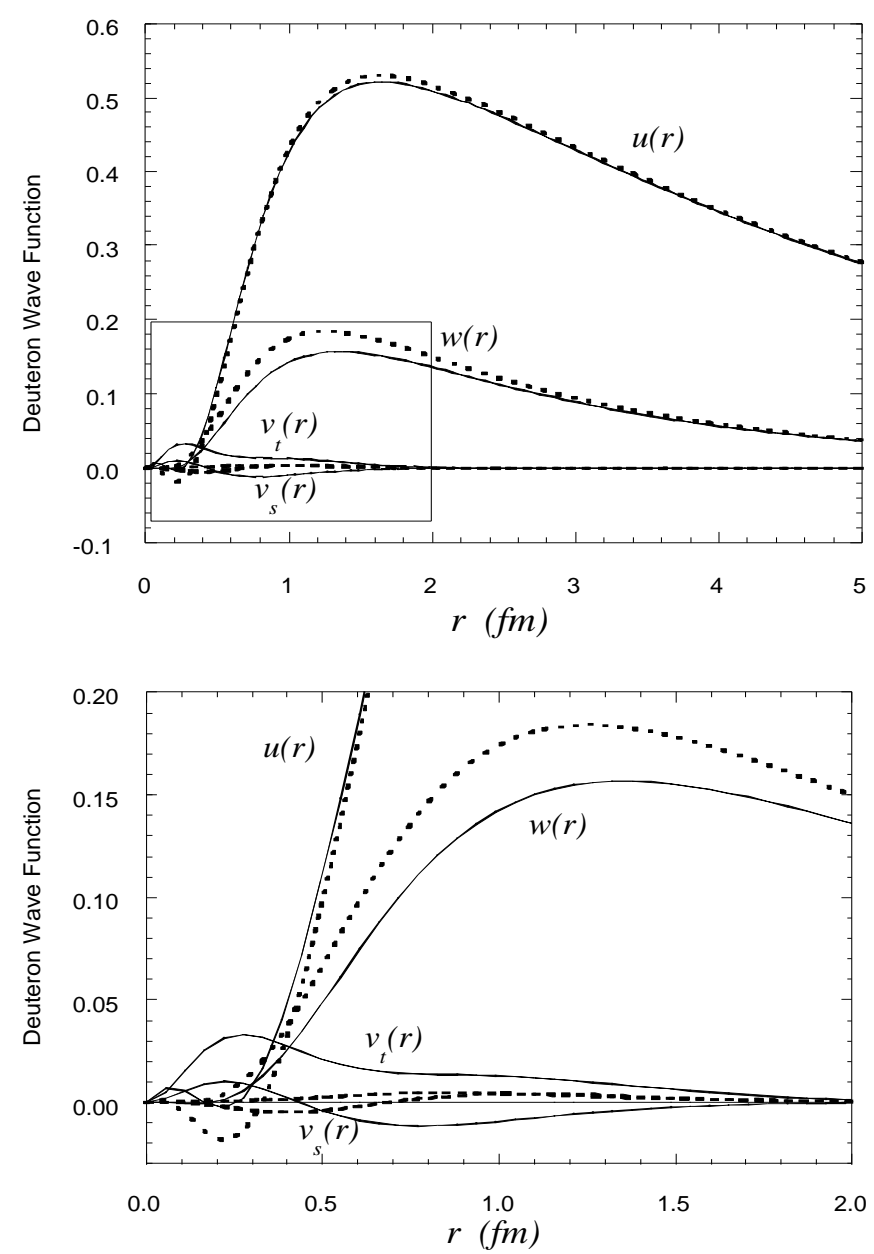

FIG. 59: A relativistic deuteron wave function. Shown is the four-component relativistic deuteron wave function of Reference [52]. The solid lines represent the wave function of model IIB, while the dotted line is that of model W16. The boxed region in the upper panel is shown in the lower panel. Note that the signs of the singlet and triplet $P$ waves are opposite after approximately $0.4 \mathrm{fm}$. 
associated with the derivative term arising from the energy dependence of the potential.

The deuteron form factors can be calculated by evaluating the Feynman diagrams shown in Figure 60. Diagram (a) of this Figure has particle 1 constrained on-shell, and can be written in the form of a matrix element of an off-shell singlenucleon current operator between two of the Gross wavefunctions described above, where the off-shell current operator is given by [53]

$$
\begin{aligned}
J^{\mu}\left(p^{\prime}, p\right) & =F_{1}\left(Q^{2}\right) f_{0}\left(p^{2}, p^{2}\right) \gamma^{\mu} \\
& +\frac{F_{2}\left(Q^{2}\right)}{2 m} h_{0}\left(p^{2}, p^{2}\right) i \sigma^{\mu \nu} q_{\nu} \\
& +F_{3}\left(Q^{2}\right) g_{0}\left(p^{\prime 2}, p^{2}\right) \frac{p^{\prime}-m}{2 m} \gamma^{\mu} \frac{\not p^{\prime}-m}{2 m},
\end{aligned}
$$

with

$$
\begin{gathered}
f_{0}\left(p^{\prime 2}, p^{2}\right) \equiv \frac{h\left(p^{2}\right)}{h\left(p^{\prime 2}\right)} \frac{m^{2}-p^{\prime 2}}{p^{2}-p^{2}}+\frac{h\left(p^{\prime 2}\right)}{h\left(p^{2}\right)} \frac{m^{2}-p^{2}}{p^{\prime 2}-p^{2}}, \\
g_{0}\left(p^{\prime 2}, p^{2}\right) \equiv\left(\frac{h\left(p^{2}\right)}{h\left(p^{\prime 2}\right)}-\frac{h\left(p^{\prime 2}\right)}{h\left(p^{2}\right)}\right) \frac{4 m^{2}}{p^{\prime 2}-p^{2}} .
\end{gathered}
$$

Here, $F_{3}\left(Q^{2}\right)$ and $h_{0}\left(p^{\prime 2}, p^{2}\right)$ are arbitrary functions subject to the constraints that $F_{3}(0)=1$ and $h_{0}\left(m^{2}, m^{2}\right)=1$. In the above equations, $h\left(p^{2}\right)$ and $h\left(p^{\prime 2}\right)$ represent the initial and final nucleon form factors, while $h_{0}\left(p^{2}, p^{2}\right)=f_{0}\left(p^{\prime 2}, p^{2}\right)$ and $F_{3}\left(Q^{2}\right)=G_{E}^{p}\left(Q^{2}\right)$ [51]. For numerical values of the nucleon form factors, the parameterization of Galster et al. [54] was employed. Diagrams (b) and (c) of Figure 60 represent situations where the virtual photon is absorbed on particle 1 and the nucleon is on-shell either before (b) or after (c) the absorption of the virtual photon. All three diagrams of Figure 60 have been found to be necessary in order to recover the correct normalization for the charge form factor when $Q^{2}=0$. This method of calculating the deuteron current matrix element from 


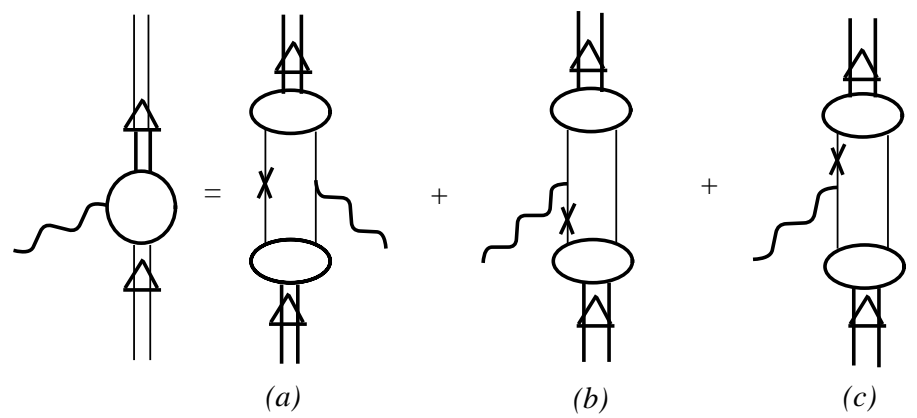

FIG. 60: Feynman diagrams representing the Gross current matrix element. The ovals represent the deuteron wave function, single lines represent nucleon propagators, x's denote the nucleon that is on-shell and the wavy lines represent virtual photons.

three diagrams (as opposed to diagram (a) only) is called the "complete impulse approximation", or CIA in later plots.

Another quasipotential approximation to the Bethe-Salpeter equation is that of Blankenbecler, Sugar, Lugunov and Tavkheidze, referred to as the BSLT equation [50]. This equation differs from the Spectator equation in that it puts both nucleons equally off-shell, so that the relative energy is set to zero. Hummel and Tjon [55] calculated the deuteron elastic form factors using the BSLT equation and a one-boson-exchange exchange kernel containing six mesons: $\pi, \eta, \sigma, \delta, \omega$ and $\rho$.

The BSLT current matrix element is described by a Feynman diagram similiar to that of Figure 60-(a) and a symmetric diagram where the virtual photon attaches to particle 1. Diagrams 60-(b) and -(c) are unnecessary because there is no on-shell constraint to account for in this approximation. In numerical calculations of the matrix element, the nucleon electromagnetic form factor parameterization of Höhler et al. [43] is employed. Since the form of the matrix element calculated in this approximation looks like a matrix element of the single-nucleon current 
operator between two relativistic wave functions, their results are referred to as the relativistic impulse approximation (RIA).

Figure 61 shows the CIA calculation of Van Orden et al. [51] and the RIA calculation of Hummel and Tjon [55] compared to this and previous data for the electric structure function $A\left(Q^{2}\right)$. The CIA calculation (solid line) gives a fair description of our data up to about $Q^{2}=1.5(\mathrm{GeV} / \mathrm{c})^{2}$, then it begins to underestimate the data, while the RIA calculation (dashed line) underestimates our data over the whole range of momentum transfers being investigated. The failure of the relativistic theories to describe the data gives an estimate of the contribution that other effects can make to the elastic electron-deuteron form factors. Additional effects which change the theoretical description of the structure functions include meson-exchange currents (Section 5.3). The theoretical description is also sensitive to the parameterization used for the single-nucleon form factors [55]. Quark effects might also be important, such as those which might arise from a possible six quark component of the deuteron wave function, so the underestimation of the data may indicate the size of any quark effects not included in meson field theory [51].

Figure 62 shows a comparison of the CIA and RIA calculations to the magnetic structure function data. Our data cover the momentum transfer range $0.7 \leq Q^{2}$ $\leq 1.35(\mathrm{GeV} / \mathrm{c})^{2}$ and is shown in this Figure as solid circles. The CIA calculation slightly overestimates our data at low $Q^{2}$ (below $\left.1.0(\mathrm{GeV} / \mathrm{c})^{2}\right)$, but describes our two highest $Q^{2}$ points. This calculation predicts a minimum in the structure function around a $Q^{2}$ of $1.8(\mathrm{GeV} / \mathrm{c})^{2}$, which is consistent with the SLAC NE4 measurement [4]. The RIA calculation (dashed line) of Hummel and Tjon underestimates the data and predicts a minimum around a $Q^{2}$ of $1.4(\mathrm{GeV} / \mathrm{c})^{2}$. Note that the minimum of $B\left(Q^{2}\right)$ is at larger $Q^{2}$ for the CIA calculation of Van Orden et al. than for the RIA calculation of Hummel and Tjon. This results from differences in the deuteron wave functions between the two models [51].

As seen above, the relativistic calculations generally underestimate the measured electric structure function, while they over- or under-estimate the magnetic 


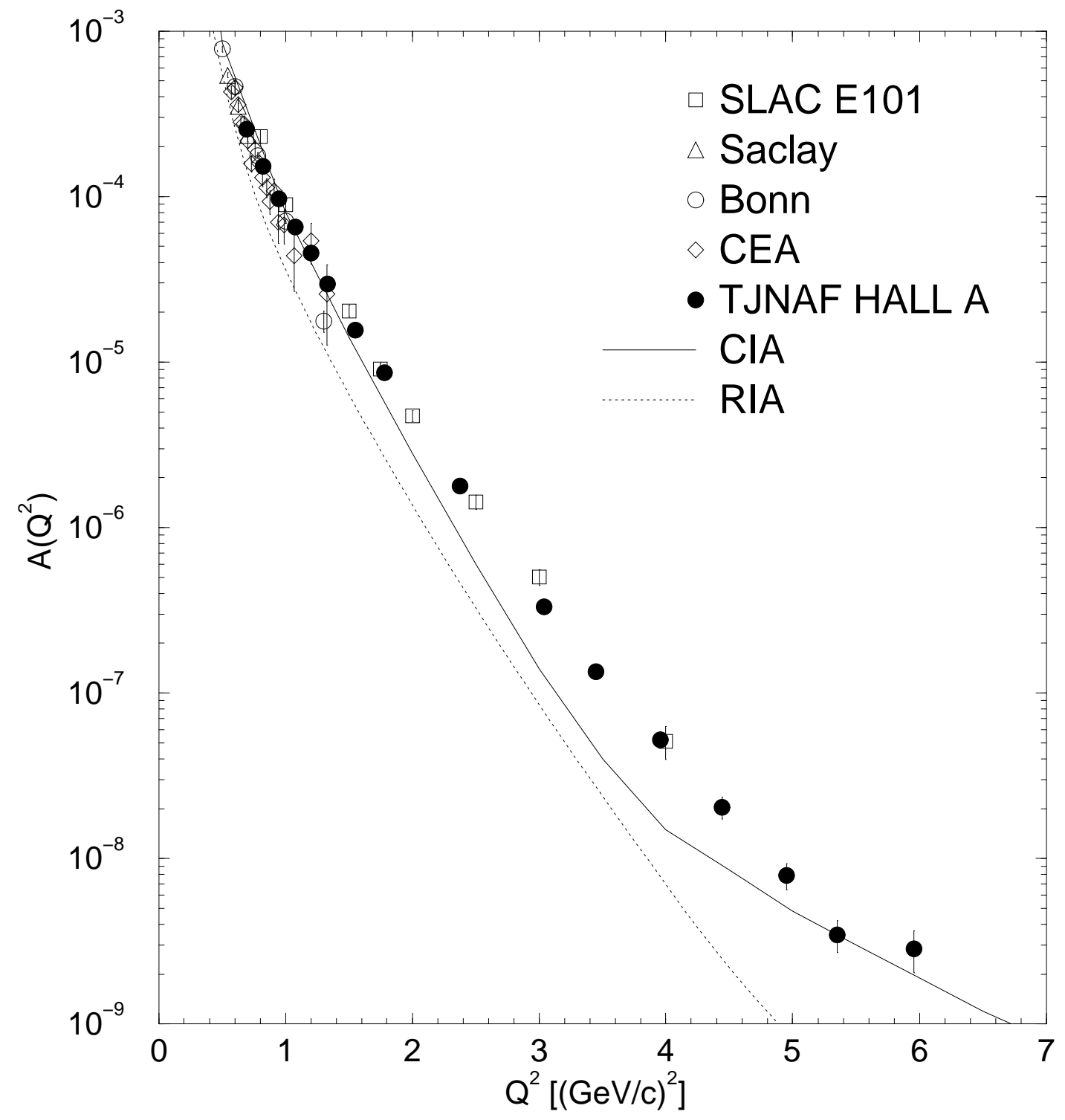

FIG. 61: Comparison of several relativistic theories to the $A\left(Q^{2}\right)$ data. Shown are the CIA calculation of Reference [51] (solid line) and RIA calculation of Reference [55] (dashed line) compared to this and previous data for $A\left(Q^{2}\right)$. The references for the data are in the text. 
structure function depending upon the model used. As discussed in the next section, the inclusion of meson exchange current contributions into the theoretical calculation of $A\left(Q^{2}\right)$ tends to bring the CIA [51] calculation into better agreement with the data, while their inclusion for $B\left(Q^{2}\right)$ leads to an underestimation of the data. The inclusion of MEC contributions into the RIA calculation [55] of Hummel and Tjon leads to an overestimation of the $A\left(Q^{2}\right)$ data and an underestimation of the $B\left(Q^{2}\right)$ data in our momentum transfer regions.

\subsection{Meson Exchange Currents}

Both nonrelativistic and relativistic impulse approximation calculations underestimate the experimentally measured values of $A\left(Q^{2}\right)$ and $B\left(Q^{2}\right)$ (see, for example, Figures 56, 57, 61 and 62). This discrepancy between theory and experiment generally increases with increasing $Q^{2}$. Since the nuclear force is mediated by the exchange of virtual mesons between nucleons in the nucleus, further contributions to the cross section can come from the scattering from these meson exchange currents (MEC). This is because the meson exchange gives rise to an electromagnetic exchange-current which is in addition to the nucleons' intrinsic magnetic moment and convection current. So, in the electromagnetic interaction of nuclei with the virtual photon, a contribution to the interaction should come from the coupling of the exchange-current with the virtual photon. MEC contributions are expected to be large at large $Q^{2}$ because they provide a mechanism for sharing the incoming photon momentum equally between the two nucleons.

Figure 63 shows the first-order MEC diagrams. The top two diagrams describe interactions with the meson-nucleon vertex (left) and intermediate nucleonantinucleon states (right). The middle two diagrams describe interactions where the meson is not emitted at the same time as the interaction occurs (time runs vertically upward). The bottom three graphs correspond to the direct coupling of the virtual photon to the exchanged meson. The leftmost of the three has the same meson on both sides of the interaction vertex. This diagram is not allowed in elastic $e-d$ scattering because it violates G-parity conservation. The remaining 


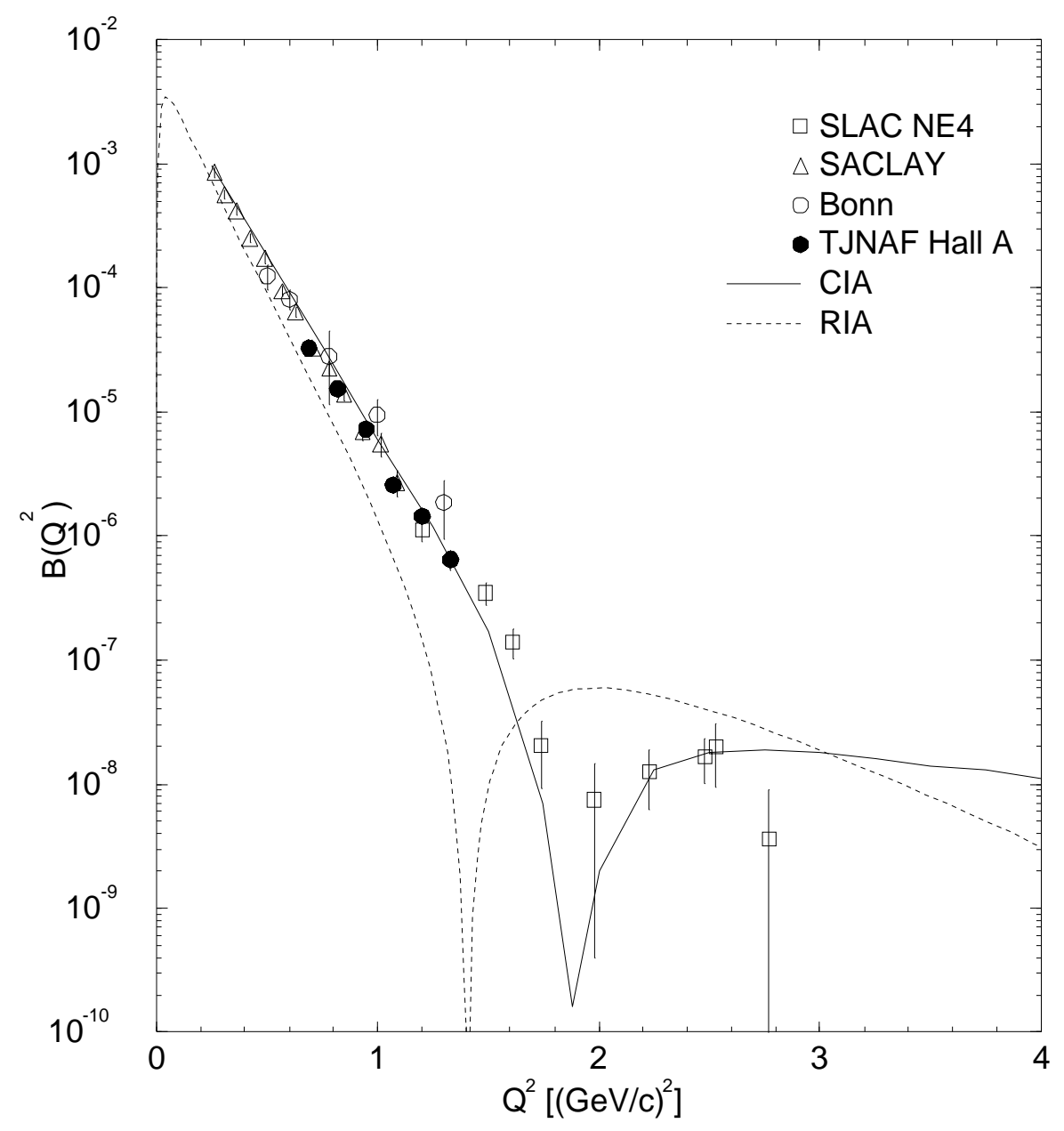

FIG. 62: Comparison of several relativistic calculations to the $B\left(Q^{2}\right)$ data. Shown are the CIA calculation of Reference [51] (solid line) and RIA calculation of Reference [55] (dashed line) compared to this and previous data for $B\left(Q^{2}\right)$. The references for the data are in the text. 
two diagrams, the " $\omega \sigma \gamma$ " and the " $\rho \pi \gamma$ " diagrams, are allowed in elastic $e-d$ scattering and are found to give a contribution to the cross section, as will be seen below.

Meson exchange contributions to the nonrelativistic impulse approximation prediction of the deuteron form factors were discussed in Section 5.1. In the relativistic Bethe-Salpeter model of the nuclear force, the form factors can be calculated from only two contributions: the relativistic impulse approximation in which the photon couples directly to one of the bound nucleons, and the meson exchange current contribution in which the photon couples to the exchanged mesons. This means that the diagrams from Rows 1 and 2 of Figure 63 are taken into account automatically in the RIA formalism, so the only MEC contributions that need to be taken into account for the deuteron form factors come from the $\rho \pi \gamma$ and $\omega \sigma \gamma$ (and related) graphs.

There are several relativistic calculations of the deuteron form factors which include MEC contribution (e.g. [55], [51]), but the magnitudes of the MEC contributions to the form factors are uncertain because of uncertainties in the mesonphoton vertex form factors and coupling constants. For the $\rho \pi \gamma$ exchange current, the coupling constant $g_{\rho \pi \gamma}$ is not well known, but it can be extracted from the decay width of $\rho \rightarrow \pi \gamma$ :

$$
g_{\rho \pi \gamma}^{2}=\frac{4 \pi}{e^{2}} \frac{24 \Gamma(\rho \rightarrow \pi \gamma)}{m_{\rho}\left(1-m_{\pi}^{2} / m_{\rho}^{2}\right)^{3}} .
$$

Experiments by Berg et al. [57] give $g_{\rho \pi \gamma}=0.56$. The sign of this coupling constant is taken to be positive. For the $\omega \sigma \gamma$ exchange current, the coupling constant is taken to be $g_{\omega \sigma \gamma}=-g_{\rho \pi \gamma}$, as suggested from a study of a relativistic quark model by Chemtob, Moniz and Rho [58], where it was found that the $g_{\rho \pi \gamma}$ and $g_{\omega \sigma \gamma}$ coupling constants are of the same magnitude, but of opposite signs.

The $Q^{2}$ dependence of the MEC vertex form factor is also uncertain. As seen in Figure 64, various parameterizations for the form factor of the $\rho \pi \gamma$ vertex exist (similarly for the $\omega \sigma \gamma$ vertex). In this figure, the solid curve is the vertex form 

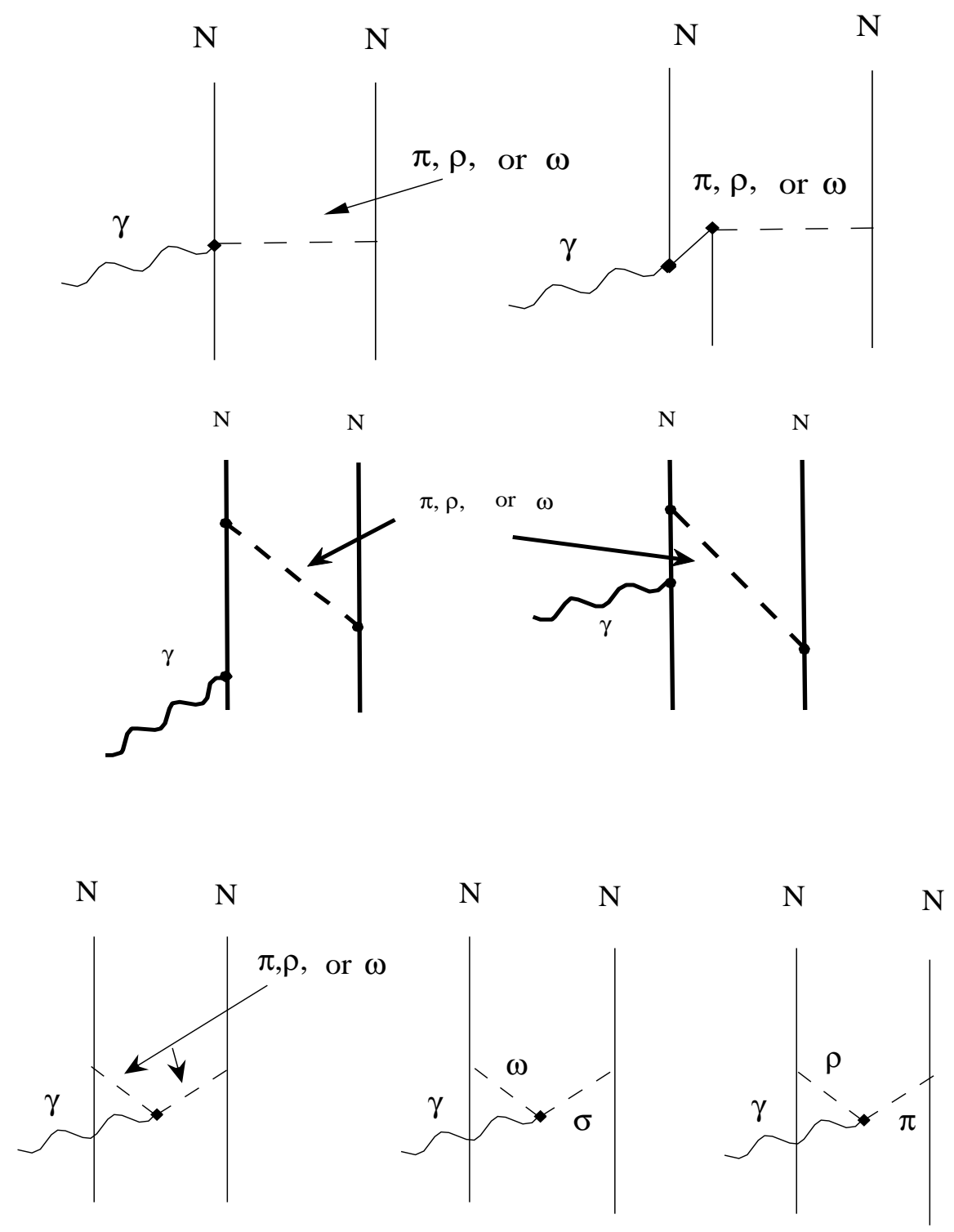

FIG. 63: Feynman diagrams of Meson Exchange Currents. Top row: seagull (left), pair (right), Middle row: Retardation graphs, Bottom row: Meson-Photon interaction graphs; the first diagram in this row doesn't contribute because of $G$-parity conservation. 


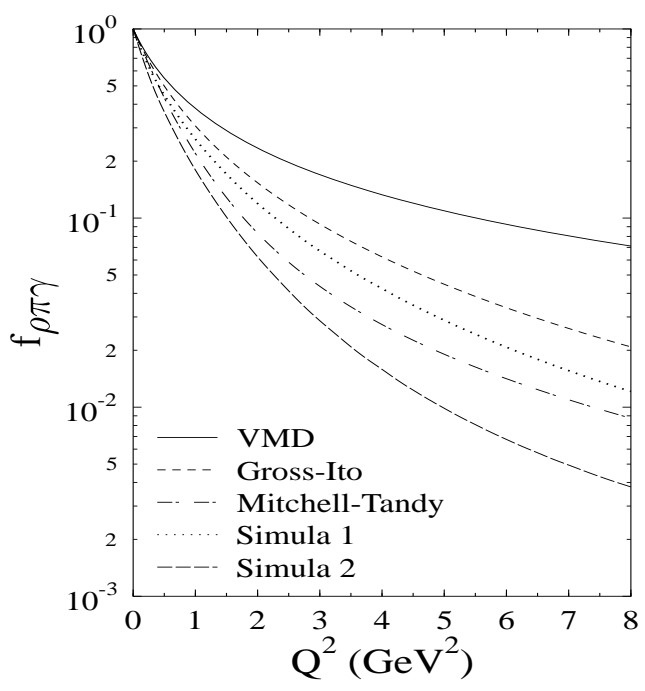

FIG. 64: Various models of the $\rho \pi \gamma$ MEC form factor.

factor obtained with the Vector Meson Dominance (VMD) model, in which the form factor is parameterized as

$$
f_{\rho \pi \gamma}\left(Q^{2}\right)=\frac{m_{\omega}^{2}}{m_{\omega}^{2}+Q^{2}},
$$

where $m_{\omega}$ is the mass of the $\omega$ meson. This model describes the interaction between the virtual photon and meson as being mediated by two vector mesons with similar masses and opposite phases. The other predictions shown in Figure 64 for the $\rho \pi \gamma$ vertex form factor are from quark loop calculations in which relativistic models of the $\rho$ and $\pi$ mesons are used. As the figure shows, the quark-based models produce a much softer form factor than is produced by the VMD assumption. The difference between the models is substantial at large $Q^{2}$, which results in a range of predictions for the $Q^{2}$ evolution of the deuteron structure functions when MEC contributions are included. 


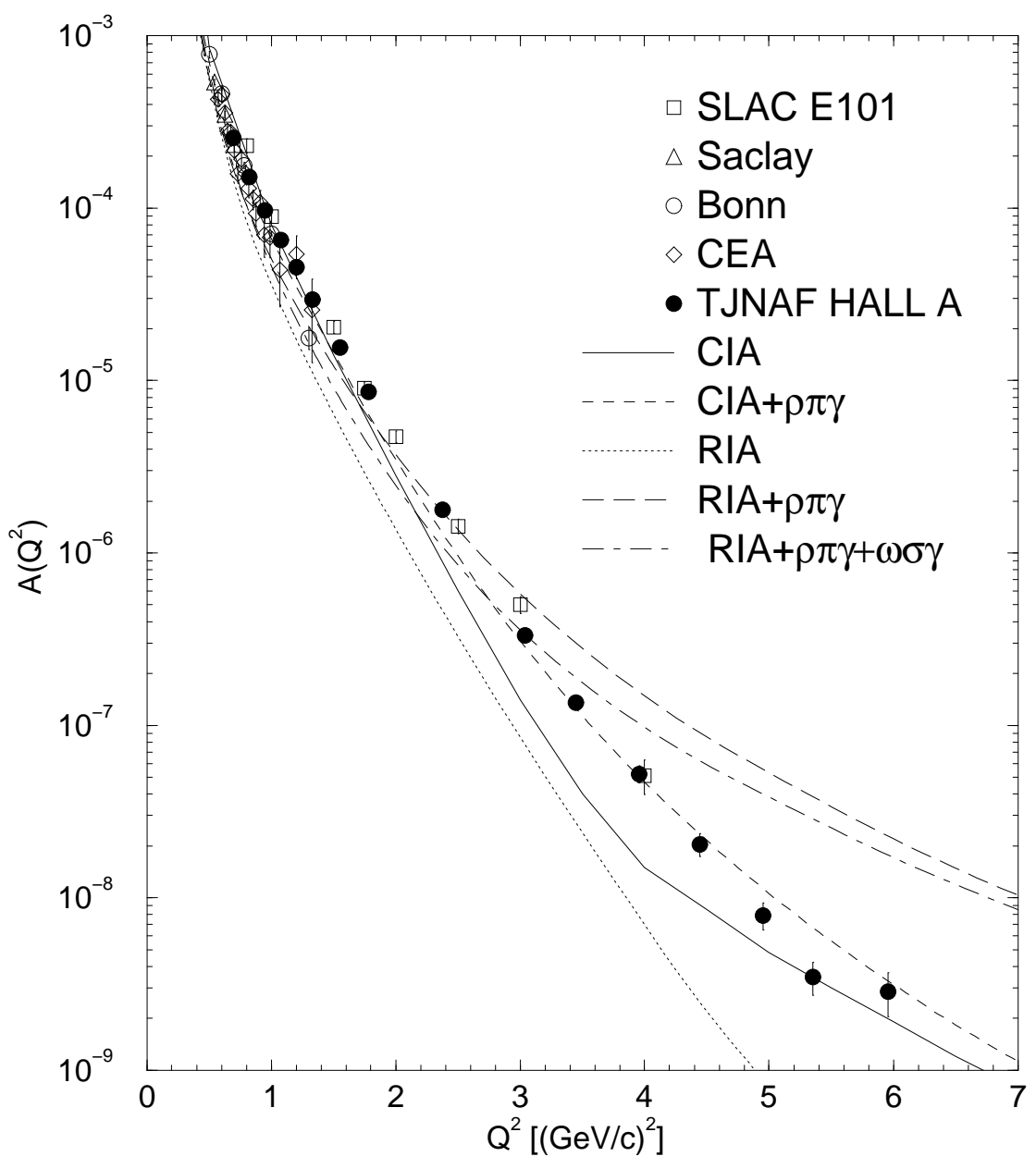

FIG. 65: Comparison of several relativistic calculation (with the inclusion of MEC) to the $A\left(Q^{2}\right)$ data. The solid line is the CIA of Van Orden et al. [51]. The dashed line is CIA $+\rho \pi \gamma$ meson exchange current. The dotted line is the RIA of Hummel and Tjon [55]. The long-dashed line is the RIA $+\rho \pi \gamma$ meson exchange current. The dot-dashed line is the RIA $+\rho \pi \gamma+\omega \sigma \gamma$ meson exchange currents. 
Figure 65 shows the RIA and RIA+MEC calculations of Hummel and Tjon [55] compared to this and previous data for the electric structure function $A\left(Q^{2}\right)$. As seen, the RIA prediction (dotted line) underestimates the data. When the $\rho \pi \gamma$ MEC contribution is included (long-dashed line), using the VMD form factor and $g_{\rho \pi \gamma}=0.56$ for the coupling constant, the data is overestimated. An additional exchange current contribution comes from including the $\omega \sigma \gamma$ MEC. Assuming that $f_{\omega \sigma \gamma} \approx$ constant $\times f_{\rho \pi \gamma}$ and $g_{\omega \sigma \gamma}=-g_{\rho \pi \gamma}$, adding the $\omega \sigma \gamma$ MEC cancels part of the $\rho \pi \gamma \mathrm{MEC}$, resulting in a prediction for $A\left(Q^{2}\right)$ that still overestimates the data (dot-dashed line), but to a lesser extent than when only the $\rho \pi \gamma$ MEC contribution is included. Also seen in this Figure are the CIA and CIA+MEC calculations of Van Orden et al. [51]. As in the case of the RIA of Hummel and Tjon, the CIA (solid line) underestimates the $A\left(Q^{2}\right)$ data. When the $\rho \pi \gamma$ MEC contribution is added (short-dashed line), using the $\rho \pi \gamma$ vertex form factor parameterization of Gross and Ito [5], the theory agrees well with the data over most of the measured $Q^{2}$ range.

Figure 66 shows a comparison between theory and data for the $B\left(Q^{2}\right)$ structure function. The RIA calculation (dotted line) of Hummel and Tjon underestimates the data and predicts a minimum around $Q^{2}$ of $1.4(\mathrm{GeV} / \mathrm{c})^{2}$. Addition of the $\rho \pi \gamma$ MEC (long-dashed line) has a small effect at high $Q^{2}$, but otherwise doesn't change the RIA results. Addition of the $\omega \sigma \gamma$ MEC (dot-dashed line) brings the prediction closer to the data, though the discrepancy is still significant. Also seen in this Figure are the CIA and CIA+MEC calculations of Van Orden et al. The CIA (solid line) calculation slightly underestimates our low $Q^{2}$ data (below $\left.1.0(\mathrm{GeV} / \mathrm{c})^{2}\right)$, but describes our two highest $Q^{2}$ points without the addition of any meson exchange current contribution. When the $\rho \pi \gamma$ MEC contribution is added, the position of the minimum shifts toward lower $Q^{2}$ and the data is underestimated.

In summary, including a contribution to the form factors from the $\rho \pi \gamma$ meson exchange current tends to increase the size of $A\left(Q^{2}\right)$ and shift the minimum of $B\left(Q^{2}\right)$. In the case of $A\left(Q^{2}\right)$, using the vector meson dominance form factor for the meson-photon vertex produces much too large an effect, while using the 
softer quark model form factors gives smaller effects. Hummel and Tjon found that a $\omega \sigma \gamma$ MEC contribution was necessary (which cancelled some of the $\rho \pi \gamma$ MEC contribution), while Van Orden et al. found that this exchange current was unnecessary.

This description of the deuteron in terms of nucleons and mesons should eventually break down at sufficiently large momentum transfers. There, a quarkbased description is known to be more economical. Furthermore, because QCD is asymptotically free, at large enough momentum transfer the strong coupling constant, $\alpha_{s}$, becomes small enough for perturbative QCD (pQCD) calculations of the asymptotic behavior of the deuteron form factors to be feasible.

\subsection{Quark Dimensional Scaling and Perturba- tive QCD}

The first predictions for the asymptotic behavior of the deuteron structure functions in terms of quarks came from dimensional counting rules [59],[60]. Using dimensional analysis, the asymptotic behavior of the cross section for the scattering process $\mathrm{AB} \rightarrow \mathrm{CD}$ is

$$
\frac{d \sigma}{d t} \sim \frac{f(t / s)}{t^{n-2}}
$$

( $t \rightarrow \infty, t / s$ fixed, where $s$ and $t$ are the Mandlestam variables). In Equation (67), $n$ is the total number of leptons $\left(n_{e}\right)$, photons and quark $\left(n_{H}\right)$ components of the initial and final states. For elastic electron-deuteron scattering, $n=14$. This equation was derived [61] starting with the observation that the invariant amplitude $M$, which connects the initial and final states, has the dimension of energy to the power $4-n$. At large energy and momentum transfer, $t$ is the energy scale available, leading to

$$
M \sim \frac{f(t / s)}{(\sqrt{t})^{n-4}} .
$$




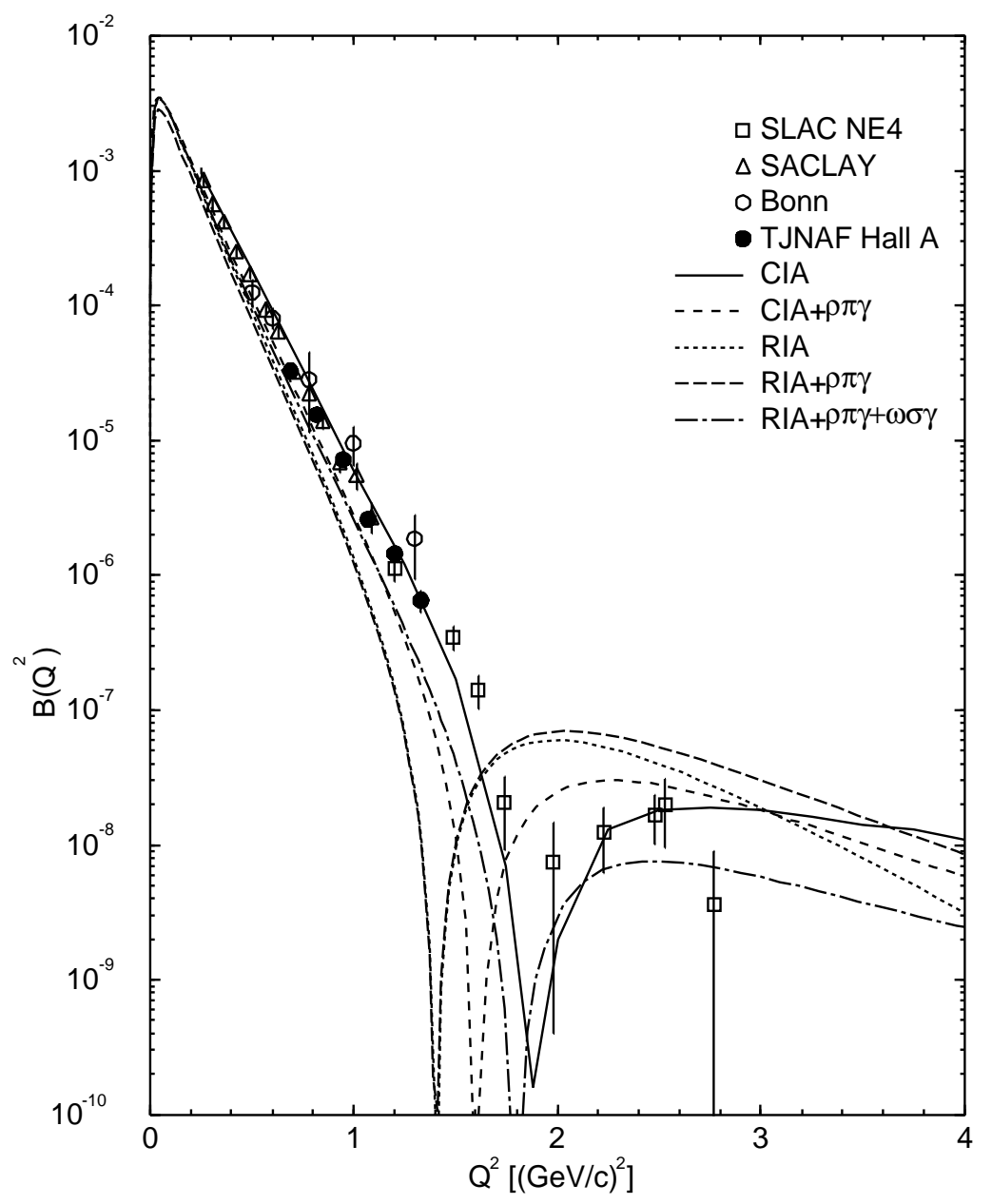

FIG. 66: Comparison of several relativistic calculation (with the inclusion of MEC) to the $B\left(Q^{2}\right)$ data. The solid line is the CIA of Van Orden et al. [51]. The dashed line is CIA $+\rho \pi \gamma$ meson exchange current. The dotted line is the RIA of Hummel and Tjon [55]. The long-dashed line is the RIA $+\rho \pi \gamma$ meson exchange current. The dot-dashed line is the RIA $+\rho \pi \gamma+\omega \sigma \gamma$ meson exchange currents. 
Since the cross section is related to the amplitude by $\frac{d \sigma}{d t} \sim|M|^{2} / t^{2}$, Equation (67) is obtained.

This result can be applied to describe the asymptotic dependence of the electromagnetic form factor of an elastic electron-hadron interaction. The cross section can be written as

$$
\frac{d \sigma}{d t} \sim \frac{\left|F\left(Q^{2}\right)\right|^{2}}{\left(Q^{2}\right)^{2}} f(t / s)
$$

Combining this with Equation (67) leads to an expression for the form factor which depends upon $n_{H}$, the number of quark constituents of the hadron:

$$
F\left(Q^{2}\right) \sim \frac{1}{\left(Q^{2}\right)^{n_{H}-1}}
$$

where $n=2 n_{H}+2 n_{e}$ and $n_{e}=1$ have been used. Applying this to mesons $\left(n_{H}=2\right)$ leads to the prediction $F\left(Q^{2}\right) \sim 1 / Q^{2}$. For the proton $\left(n_{H}=3\right)$, it gives $F_{1}\left(Q^{2}\right) \sim 1 /\left(Q^{2}\right)^{2}$ for the dominant form factor and $F_{2}\left(Q^{2}\right) \sim 1 /\left(Q^{2}\right)^{3}$. The magnetic form factor is suppressed by a factor of $1 / Q^{2}$ because one quark helicity flip is required in magnetic scattering. Applying the above to elastic electrondeuteron $\left(n_{H}=6\right)$ scattering leads to a prediction for the asymptotic behavior of the dominant form factor $A\left(Q^{2}\right)$ :

$$
A\left(Q^{2}\right) \sim\left(\frac{1}{Q^{2}}\right)^{10}
$$

and for the magnetic form factor $B\left(Q^{2}\right)$ :

$$
B\left(Q^{2}\right) \sim\left(\frac{1}{Q^{2}}\right)^{12}
$$



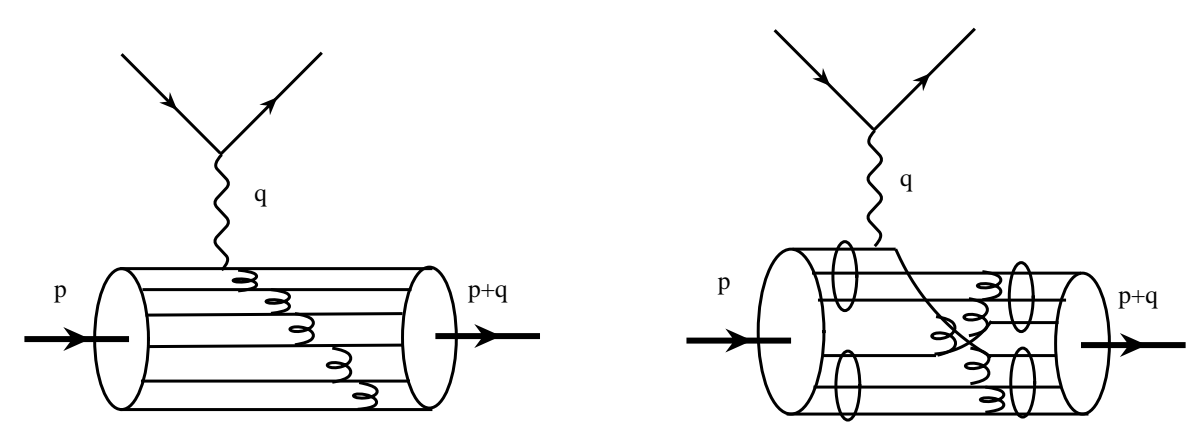

FIG. 67: Schematic of quark momentum exchange during elastic scattering. The horizontal solid lines represent the quarks. The squiggly lines between the quarks represent the exchange of gluons. The left diagram is called a cascade diagram, while the right diagram is known as a quark interchange diagram.

These predictions were later substantiated in perturbative QCD, as described below.

Quantum chromodynamics is the theory of the strong interaction, so it should be able to provide a fundamental description of nuclear physics. Thus, even if phenomena appear to be explained by the traditional nuclear physics of nucleons and mesons, they should also, in principle, be explainable in terms of quarks and gluons. As seen in previous sections, the deuteron elastic electric and magnetic structure functions have been fairly well described by the relativistic impulse approximation formalism, when meson exchange current contributions are included (Figures 65,66 ). As described below, the pQCD description of the asymptotic behavior of the electric structure function agrees well with the data beyond $Q^{2} \sim 4$ $(\mathrm{GeV} / \mathrm{c})^{2}$. 
To get the $Q^{2}$ dependence of the form factors at high $Q^{2}$, the deuteron is considered as a collection of six parallel-moving quarks [62], [63], as in Figure 67. The scattering process is described by one of the quarks absorbing a virtual photon of momentum $q\left(Q^{2}=-q^{2}\right)$. This momentum is then shared equally among the six quarks, via the exchange of hard (energetic) gluons. In this picture, the prediction for the dominant form factor, $F_{d} \equiv \sqrt{A\left(Q^{2}\right)}$, has the form [62]

$$
F_{d}\left(Q^{2}\right)=\left[\frac{\alpha_{s}\left(Q^{2}\right)}{Q^{2}}\right]^{5} \sum_{m, n} d_{m n}\left(\ln \frac{Q^{2}}{\Lambda_{Q C D}^{2}}\right)^{-\gamma_{n}^{d}-\gamma_{m}^{d}}
$$

where $\alpha_{s}\left(Q^{2}\right)$ and $\Lambda_{Q C D}$ are the QCD strong coupling constant and scale parameter, and $\gamma_{m, n}$ and $d_{m n}$ are QCD anomalous dimensions and constants. The main dependence $\left[\frac{\alpha_{s}\left(Q^{2}\right)}{Q^{2}}\right]^{5}$ comes from the five quark-gluon vertices. This prediction is consistent with that of the dimensional counting rules described above. This prediction also is seen (Figure 68) to be consistent with the data above a momentum transfer squared of about $4(\mathrm{GeV} / \mathrm{c})^{2}$.

In order to approximately remove the effects of nucleon compositeness, a new "reduced" deuteron form factor, $f_{d}$, was defined [62]:

$$
f_{d}\left(Q^{2}\right) \equiv \frac{F_{d}\left(Q^{2}\right)}{F_{N}^{2}\left(Q^{2} / 4\right)}
$$

where $F_{N}\left(Q^{2}\right)=\left(1+Q^{2} / 0.71\right)^{-2}$ is the dipole form factor. The arguments for the nucleon form factors $\left(F_{N}\right)$ are $Q^{2} / 4$ because in the limit of zero binding energy, each nucleon must change its momentum by $Q / 2$. The reduced nuclear form factor is directly related to the probability for the two nucleon system to remain intact. In terms of the reduced deuteron form factor, the pQCD prediction for the falloff of $A\left(Q^{2}\right)$ is

$$
f_{d}\left(Q^{2}\right)=\frac{\alpha_{s}\left(Q^{2}\right)}{Q^{2}}\left(\ln \frac{Q^{2}}{\Lambda_{Q C D}^{2}}\right)^{(2 / 5) c_{F} / \beta},
$$




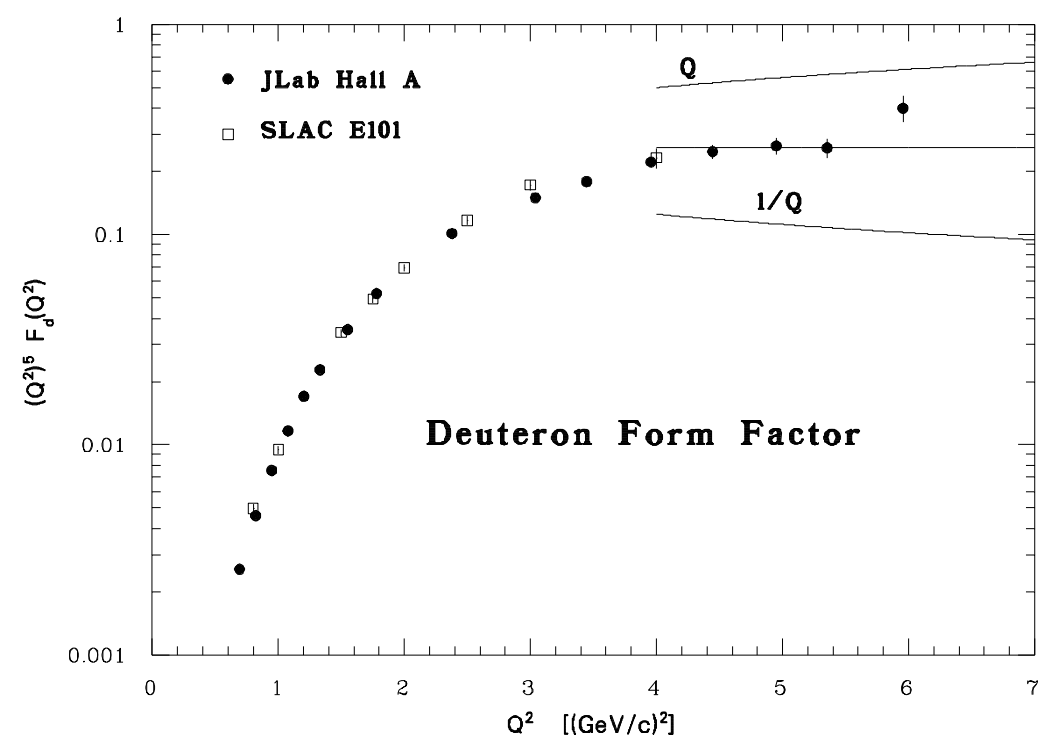

FIG. 68: Deuteron form factor scaling. $F_{d} \equiv \sqrt{A\left(Q^{2}\right)} \times\left(Q^{2}\right)^{5}$ from this experiment [6] and from SLAC E101 [3]. The line labeled $Q$ shows how the data would scale if $F_{d}\left(Q^{2}\right) \sim 1 / Q^{9}$; the line labelled $1 / Q$ shows how the data would scale if $F_{d}\left(Q^{2}\right) \sim 1 / Q^{11}$, while the straight line shows the shape for the predicted $1 / Q^{10}$ scaling. 
where $c_{F}=\left(n_{c}^{2}-1\right) / n_{c}$ and $\beta=11-(2 / 3) n_{f}$, with $n_{c}=3$ and $n_{f}=2$ being the number of colors and effective flavors. Our data appear to follow this prediction for $Q^{2}>2(\mathrm{GeV} / \mathrm{c})^{2}$ (Figure 69$)$.

The predictions described above are valid for $Q^{2} \gg 2 M_{d} \Lambda_{Q C D}$. For example, when $\Lambda_{Q C D}=200 \mathrm{MeV}$, this condition becomes $Q^{2} \gg \sim 0.8(\mathrm{GeV} / \mathrm{c})^{2}$. Although these predictions seem to be valid at our momentum transfers of approximately 3-6 $(\mathrm{GeV} / \mathrm{c})^{2}$, pQCD predictions for the asymptotic behavior of the deuteron tensor polarization, $T_{20}$, are not supported by the data in this same momentum transfer range [61].

Several authors have questioned the validity of pQCD at the momentum transfers of this experiment. In Reference [64], Isgur et al. concluded that, at these momentum transfers, nonperturbative contributions dominate the form factors and the pQCD formalism is not applicable until much larger momentum transfers. In addition, Farrar et al. [65] obtained the absolute normalization for the pQCD prediction of the reduced deuteron form factor shown in Figure 69. It was found to be almost four orders of magnitude smaller than experiment at $Q^{2} \sim 4$ $(\mathrm{GeV} / \mathrm{c})^{2}$ (note that the curve in Figure 69 has been normalized to the data at $Q^{2}$ $\left.=4(\mathrm{GeV} / \mathrm{c})^{2}\right)$. This suggests that if pQCD is to be applicable at this momentum transfer range, additional corrections must be taken into account. 


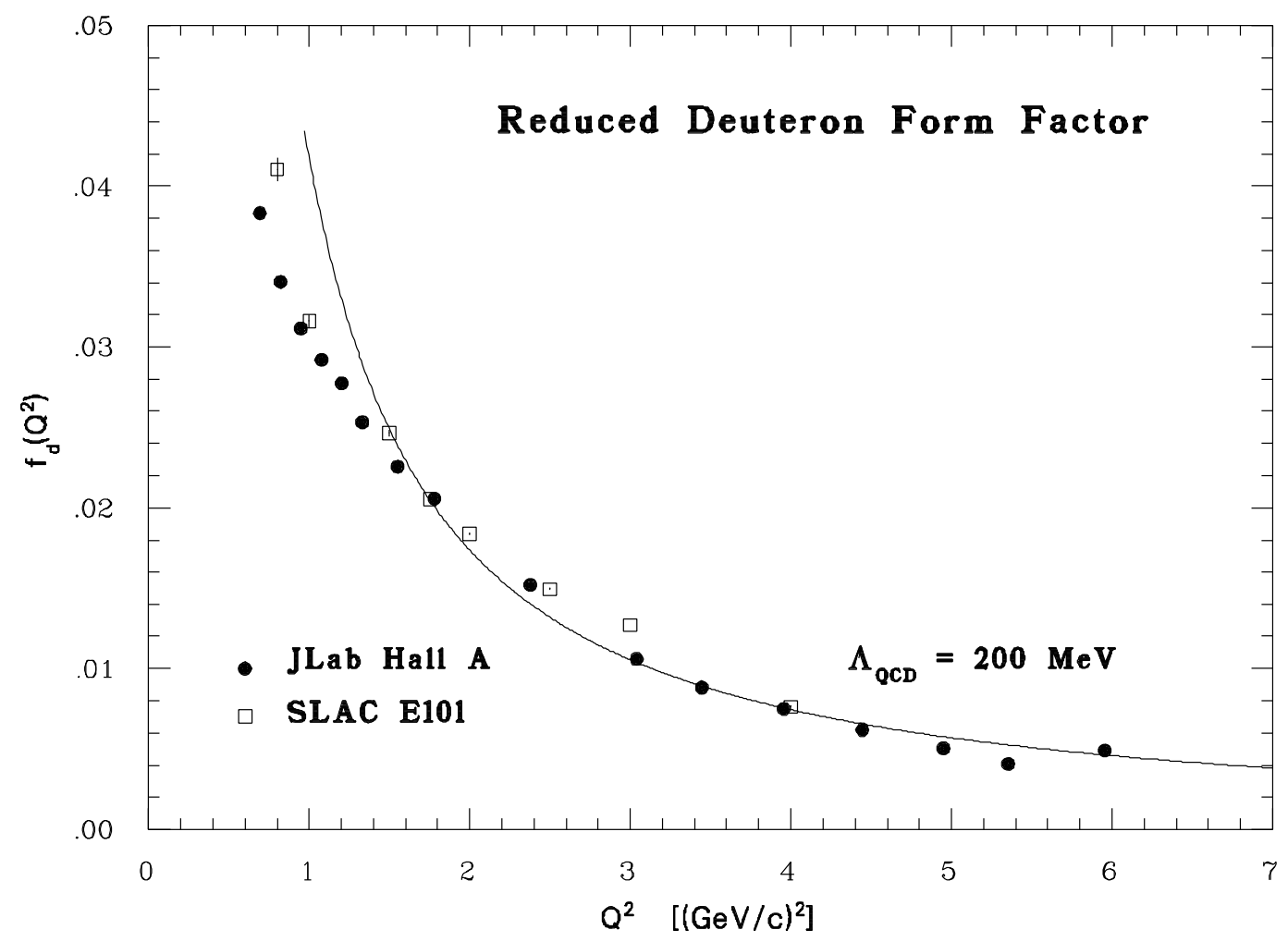

FIG. 69: The reduced deuteron form factor, $f_{d} \equiv \frac{F_{d}\left(Q^{2}\right)}{F_{N}^{2}\left(Q^{2} / 4\right)}$, as a function of $Q^{2}$. The curve is the asymptotic pQCD prediction of Ref. [62] with $\Lambda_{Q C D}=200 \mathrm{MeV}$, arbitrarily normalized to the data at $Q^{2}=4(\mathrm{GeV} / \mathrm{c})^{2}$. 


\section{Chapter 6}

\section{SUMMARY AND CONCLUSIONS}

We have performed a precise measurement of the deuteron elastic structure functions $A\left(Q^{2}\right)$ and $B\left(Q^{2}\right)$, in the momentum transfer ranges of $0.7 \leq Q^{2} \leq 6.0$ $(\mathrm{GeV} / \mathrm{c})^{2}$ and $0.7 \leq Q^{2} \leq 1.35(\mathrm{GeV} / \mathrm{c})^{2}$, respectively. This measurement took place in Hall A at Jefferson Laboratory. The structure functions were measured via coincidence elastic electron-deuteron scattering of the JLAB electron beam from a high power liquid deuterium target. More than 700 Watts of power was deposited on the target from the electron beam, enabling a cross section measurement at the level of $10^{-41} \mathrm{~cm}^{2} / \mathrm{sr}$.

The results of the structure function measurements were compared to calculations based on nonrelativistic (NRIA) and relativistic (RIA) impulse approximation predictions, both with and without the inclusion of meson exchange currents (MEC). Both NRIA and RIA predictions generally underestimated the structure functions until MEC contributions were added. The effect of the meson exchange current contributions to the structure functions varied according to the model used for the vertex form factors and coupling constants. Which meson exchange current contributions are important is also uncertain, as some groups saw the need to include several different MEC mechanisms and others did not. Both of the preceding observations indicate that our understanding of elastic electron deuteron 
scattering is still incomplete.

The results of the $A\left(Q^{2}\right)$ measurement were compared to the predictions of the dimensional scaling quark model and to perturbative Quantum Chromodynamics. These models predict that, at large momentum transfer, the structure function should fall off as $1 /\left(Q^{2}\right)^{10}$. In this case, the product $A\left(Q^{2}\right) \times\left(Q^{2}\right)^{10}$ should be independent of $Q^{2}$ (i.e. $A\left(Q^{2}\right)$ should exhibit a scaling behavior). Although the validity of $\mathrm{pQCD}$ at these moderate momentum transfers has been questioned [64], [65], our data exhibit this scaling behavior beyond a momentum transfer of approximately $4(\mathrm{GeV} / \mathrm{c})^{2}$. The origin of this behavior provides a challenge to theory and indicates the presence of interesting phenomena in the deuteron, even at moderate momentum transfers. 


\section{Appendix A}

\section{Full Trigger Electronics Diagram}




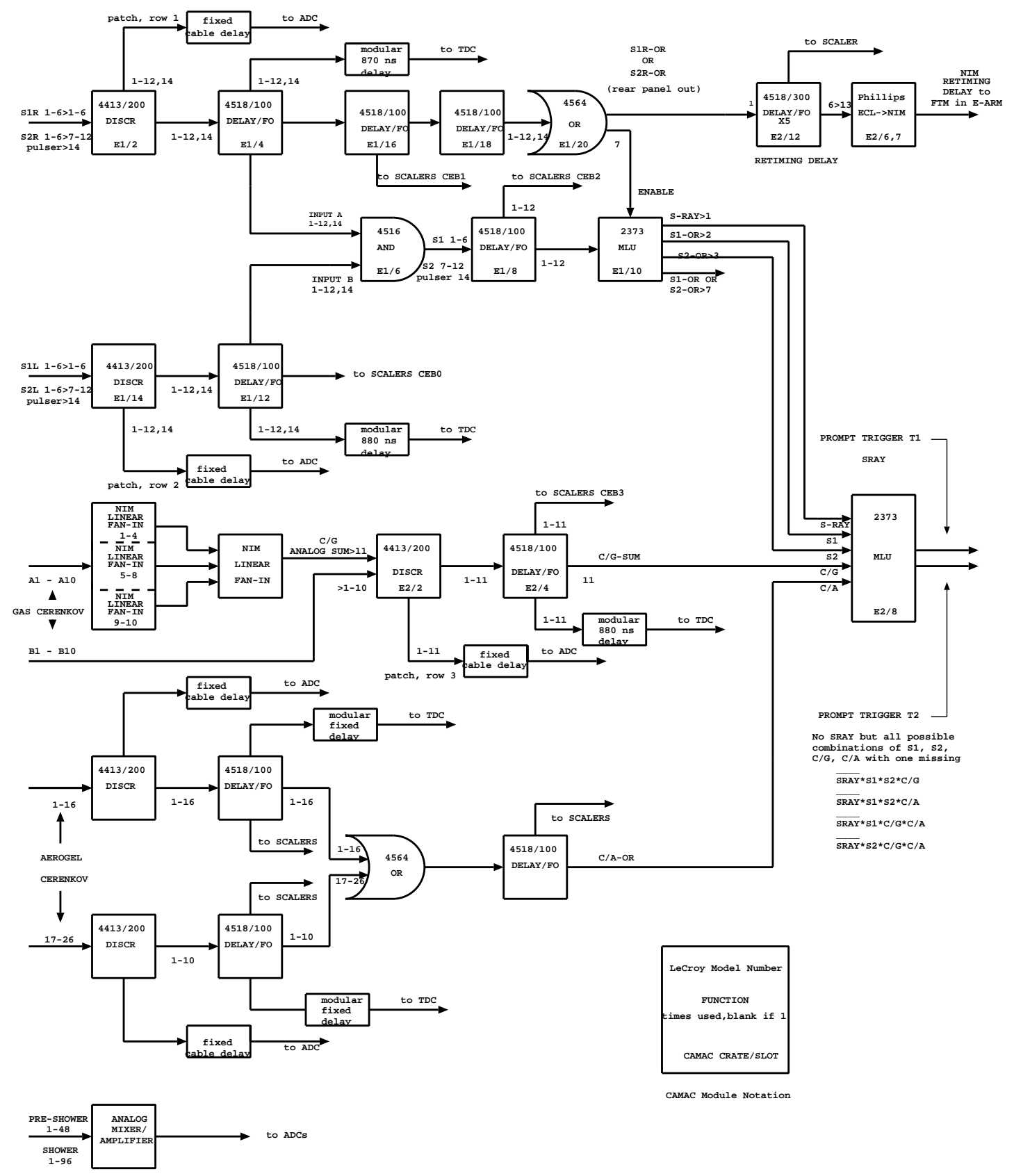

HALI A ELECTRON PROMPT TRIGGER DESIGN 11/13/97

FIG. 70: Electron Spectrometer Trigger Circuit. 


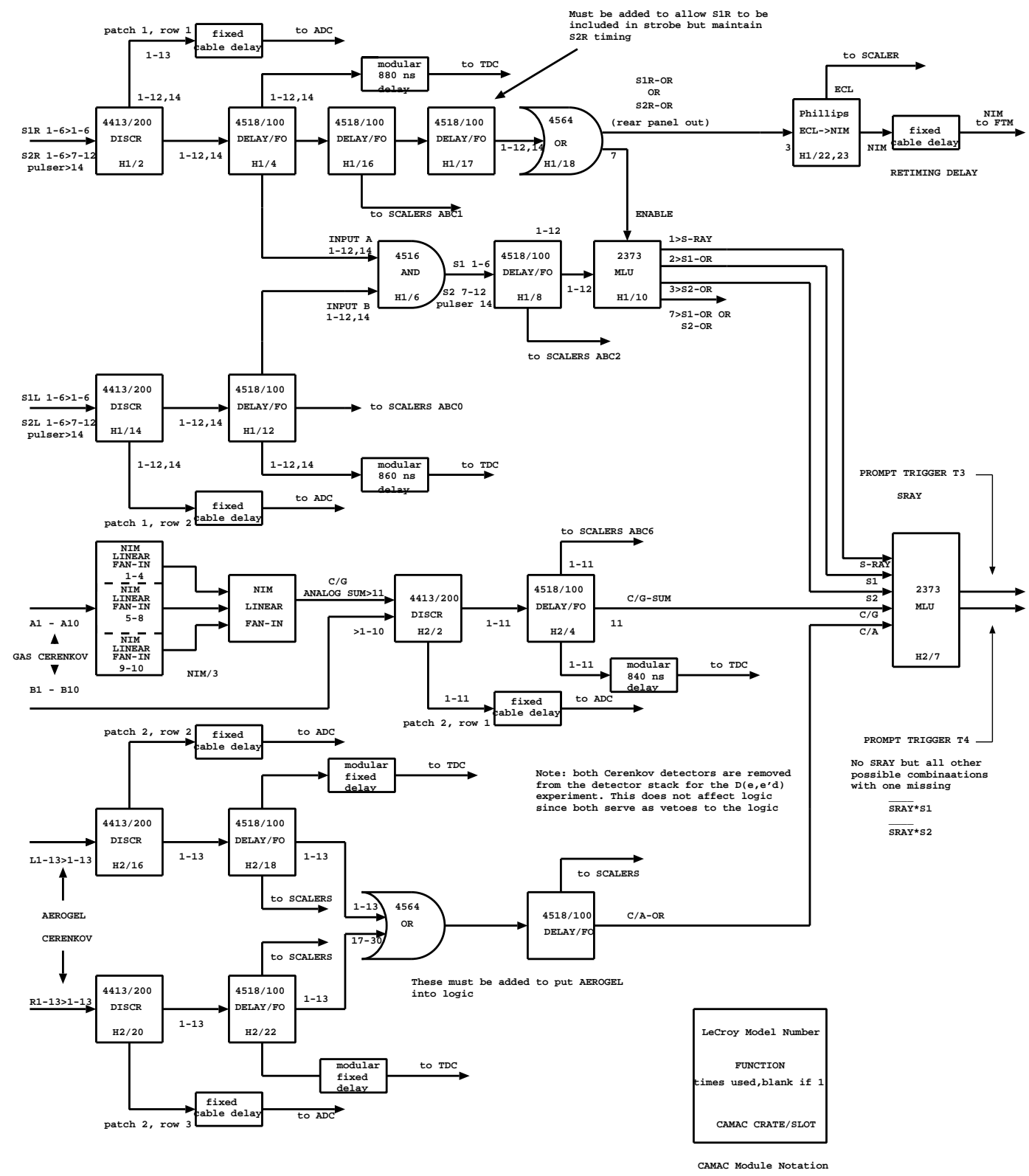

Note: The FPP is not included in TRIGGER LOGIC

Note: The modular delays are NIM LD1200

HALL A HADRON PROMPT TRIGGER DESIGN 11/13/97

FIG. 71: Hadron Spectrometer Trigger Circuit. 


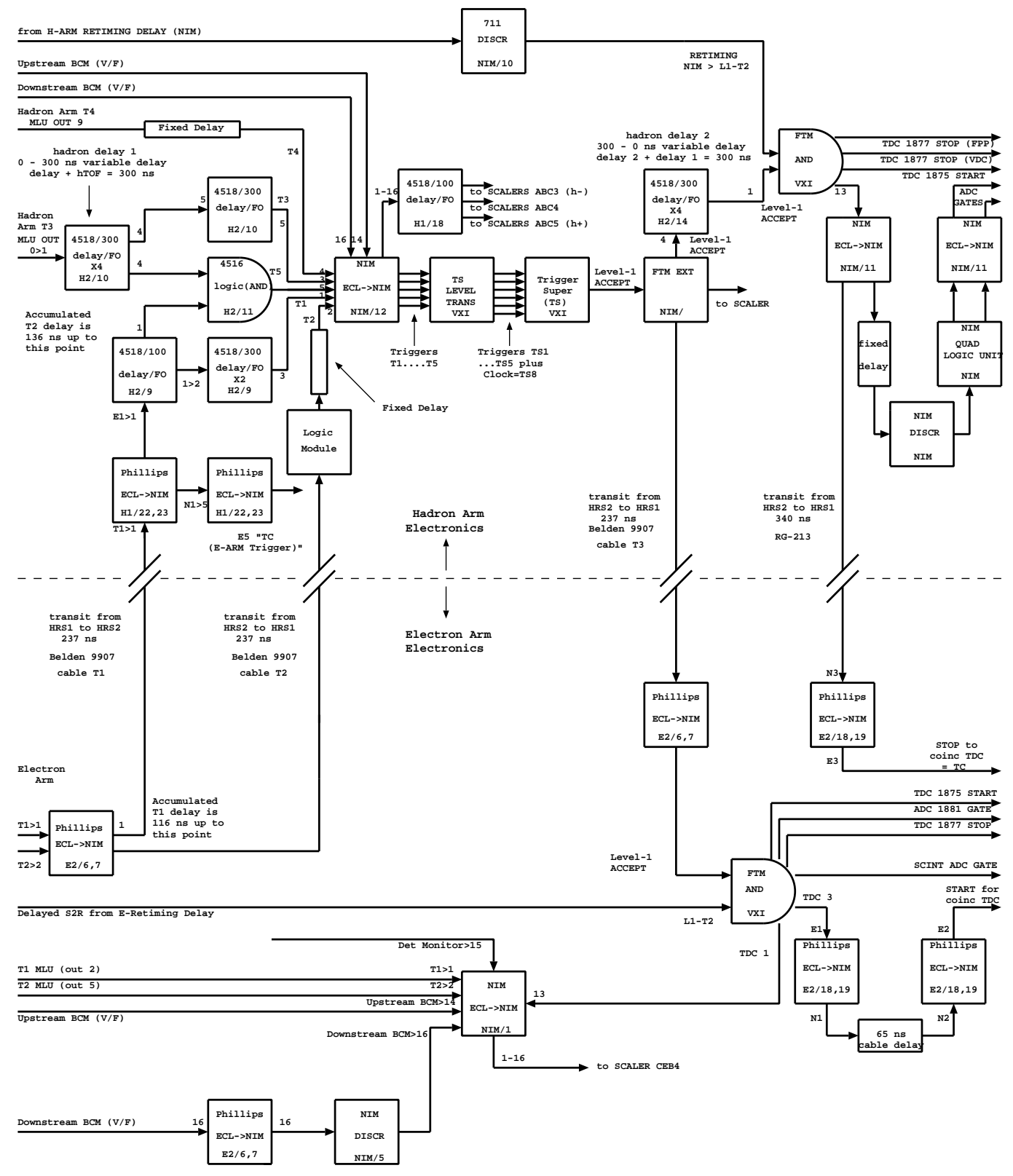

Coincidence Trigger $11 / 14 / 97$

FIG. 72: Coincidence Trigger Circuit. 


\section{Appendix B}

\section{Tables of Kinematics}


TABLE XV: Kinematics for the $A\left(Q^{2}\right)$ measurements.

\begin{tabular}{|c|c|c|}
\hline $\begin{array}{c}Q^{2} \\
(\mathrm{GeV} / \mathrm{c})^{2}\end{array}$ & $\begin{array}{c}\theta_{e} \\
(\text { degrees })\end{array}$ & $\begin{array}{c}\text { Nominal Beam Energy } \\
(\mathrm{GeV})\end{array}$ \\
\hline \hline 0.693 & 15.22 & 3.245 \\
\hline 0.821 & 16.67 & 3.245 \\
\hline 0.948 & 18.03 & 3.245 \\
\hline 1.076 & 19.32 & 3.245 \\
\hline 1.203 & 20.56 & 3.245 \\
\hline 1.331 & 21.77 & 3.245 \\
\hline 1.550 & 23.77 & 3.245 \\
\hline 1.780 & 25.79 & 3.245 \\
\hline 2.377 & 30.82 & 3.245 \\
\hline 3.040 & 27.98 & 4.045 \\
\hline 3.446 & 33.25 & 3.746 \\
\hline 3.956 & 36.59 & 3.746 \\
\hline 4.445 & 36.24 & 4.045 \\
\hline 4.951 & 39.30 & 4.045 \\
\hline 5.351 & 37.18 & 4.424 \\
\hline 5.357 & 41.83 & 4.045 \\
\hline 5.954 & 40.56 & 4.424 \\
\hline 5.957 & 45.74 & 4.045 \\
\hline
\end{tabular}


TABLE XVI: Table of $\mathrm{H}\left(e, e^{\prime} p\right)$ kinematics for the $A\left(Q^{2}\right)$ measurements.

\begin{tabular}{|c|c|c|c|}
\hline $\begin{array}{c}\text { Nominal } e-d Q^{2} \\
(\mathrm{GeV} / \mathrm{c})^{2}\end{array}$ & $\begin{array}{c}\text { Actual } e-p Q^{2} \\
(\mathrm{GeV} / \mathrm{c})^{2}\end{array}$ & $\begin{array}{c}\theta_{e} \\
(\text { degrees })\end{array}$ & $\begin{array}{c}\text { Nominal Beam Energy } \\
(\mathrm{GeV})\end{array}$ \\
\hline \hline 0.70 & 0.812 & 17.25 & 3.245 \\
\hline 0.83 & 0.903 & 18.358 & 3.245 \\
\hline 0.96 & 0.990 & 19.391 & 3.245 \\
\hline 1.08 & 1.072 & 20.35 & 3.245 \\
\hline 1.22 & 1.151 & 21.27 & 3.245 \\
\hline 1.35 & 1.228 & 22.14 & 3.245 \\
\hline 1.57 & 1.352 & 23.54 & 3.245 \\
\hline 1.80 & 1.477 & 24.94 & 3.245 \\
\hline 2.40 & 1.781 & 28.39 & 3.245 \\
\hline 3.10 & 2.223 & 25.47 & 4.045 \\
\hline 3.50 & 2.373 & 29.41 & 3.746 \\
\hline 4.0 & 2.616 & 31.64 & 3.746 \\
\hline 4.5 & 2.885 & 31.003 & 4.045 \\
\hline 5.0 & 3.107 & 33.07 & 4.045 \\
\hline 5.37 & 3.285 & 34.75 & 4.045 \\
\hline 5.43 & 3.354 & 31.172 & 4.424 \\
\hline 6.0 & 3.625 & 33.40 & 4.424 \\
\hline
\end{tabular}

TABLE XVII: Kinematics for the $B\left(Q^{2}\right)$ measurements.

\begin{tabular}{|c|c|c|}
\hline $\begin{array}{c}Q^{2} \\
(\mathrm{GeV} / \mathrm{c})^{2}\end{array}$ & $\begin{array}{c}\theta_{e} \\
(\text { degrees })\end{array}$ & $\begin{array}{c}\text { Nominal Beam Energy } \\
(\mathrm{GeV})\end{array}$ \\
\hline \hline 0.691 & 144.55 & 0.5423 \\
\hline 0.820 & 144.55 & 0.6014 \\
\hline 0.949 & 144.53 & 0.6580 \\
\hline 1.079 & 144.53 & 0.7122 \\
\hline 1.208 & 144.53 & 0.7649 \\
\hline 1.337 & 144.53 & 0.8159 \\
\hline
\end{tabular}


TABLE XVIII: Table of $\mathrm{H}\left(e, e^{\prime} p\right)$ kinematics for the $B\left(Q^{2}\right)$ measurements.

\begin{tabular}{|c|c|c|c|}
\hline $\begin{array}{c}\text { Nominal } e-d Q^{2} \\
(\mathrm{GeV} / \mathrm{c})^{2}\end{array}$ & $\begin{array}{c}\text { Actual } e-p Q^{2} \\
(\mathrm{GeV} / \mathrm{c})^{2}\end{array}$ & $\begin{array}{c}\theta_{e} \\
(\text { degrees })\end{array}$ & $\begin{array}{c}\text { Nominal Beam Energy } \\
(\mathrm{GeV})\end{array}$ \\
\hline \hline 0.70 & 0.369 & 90.01 & 0.5423 \\
\hline 0.83 & 0.437 & 90.00 & 0.6014 \\
\hline 0.96 & 0.505 & 89.99 & 0.6580 \\
\hline 1.08 & 0.572 & 89.98 & 0.7122 \\
\hline 1.22 & 0.639 & 90.00 & 0.7649 \\
\hline 1.35 & 0.707 & 89.99 & 0.8159 \\
\hline
\end{tabular}




\section{Bibliography}

[1] J. Carlson and R. Schiavilla, Rev. Mod. Phys. 70, 743 (1998); and references therein.

[2] D. Abbott et al., Phys. Rev. Lett. 82, 1379 (1999).

[3] R. G. Arnold et al., Phys. Rev. Lett. 35, 776 (1975).

[4] R. G. Arnold et al., Phys. Rev. Lett. 58, 1723 (1987); P. E. Bosted et al., Phys. Rev. C42, 38 (1990); and references therein.

[5] H. Ito and F. Gross, Phys. Rev. Lett. 71, 2555 (1993).

[6] L. C. Alexa et al., Phys. Rev. Lett. 82, 1374 (1999).

[7] S. Platchkov et al., Nucl. Phys. A510, 740 (1990).

[8] J. E. Elias et al., Phys. Rev. 177, 2075 (1969).

[9] R. Cramer et al., Z. Phys. C29, 513 (1985).

[10] S. Auffret et al., Phys. Rev. Lett. 54, 649 (1985).

[11] M. Petratos, CEBAF PR-91-026, (1991).

[12] Hall A, http://www.jlab.org/Hall-A

[13] C. Hyde-Wright, Event-by-Event Calibration of Beam Positions during VCS Exp. 93-050, JLAB Internal Report (unpublished). 
[14] K. Unser, IEEE Trans. Nucl. Sci. 16, 934 (1969); K. Unser, IEEE Trans. Nucl. Sci. 28, 2344 (1981).

[15] S. Nanda, ed., Hall A Experimental Equipment Operations Manual, http://www.jlab.org/Hall-A/document/OPMAN/index.html, (April 1997).

[16] L. Ewell, Charge and Current Measurements for Experiment e91026 at TJNAF, JLAB Internal Report (unpublished).

[17] A. Deur, La cible cryogenique du hall A du Thomas Jefferson National Accelerator Facility, Universite Blaise Pascal internal report (unpublished).

[18] R. Suleiman, Hall A Cryogenic and Dummy Targets Information, JLAB Internal Report (unpublished).

[19] B. Dalesio, Experimental Physics and Industrial Control System (EPICS) Overview, http://www.atdiv.lanl.gov/aot8/epics/training/New/ EPICS_Overview/index.htm (May 1998).

[20] K. McCormick, TJNAF Hall A Cryotarget Density Dependence on Beam Current, JLAB Internal Report (unpublished).

[21] J. LeRose, Latest Best Estimate of Target to Collimator/Sieve Distance, http://www.jlab.org/Hall-A/news/minutes/collimator-distance.html (March 1999).

[22] K. G. Fissum et al., VDC Manual V2.1, MIT-LNS Internal Report \#0397 (unpublished).

[23] M. Iodice et al., Nucl. Instrum. Meth. A411, 223 (1998).

[24] J. Gomez, Shower Counter for the SLAC $8 \mathrm{GeV} / \mathrm{c}$ Spectromter, SLAC Internal Report (unpublished).

[25] CODA: CEBAF On-line Data Acquisition User's Manual, JLAB Internal Report (unpublished). 
[26] R. Michaels, Online Codes Dataspy and DHIST for Hall A Spectrometers, http://www.jlab.org/Hall-A/equipment/daq/dplot.html (Jan. 1999)

[27] R. Michaels, Hall A Raw Data Structure, http://www.jlab.org/Hall-A/equipment/daq/dstruct.html (Jan. 1999)

[28] E. A. J. M. Offermann and M. Kuss, ESPACE Manual Version 2.7.0, 1998 (unpublished).

[29] L. Todor, Private Communication.

[30] N. Liyanage, Ph.D. thesis, Massachusetts Institute of Technology, 1999.

[31] J. Gao, Ph.D. thesis, Massachusetts Institute of Technology, 1999.

[32] J. Gao et al, Beam Energy Measurement at Jefferson Lab Using the Hall A Spectrometers, JLAB Internal Report (unpublished).

[33] C. Group, PAW (Physics Analysis Workstation) Users Guide, Program Library Q121, CERN.

[34] P. Ulmer, Beam Charge Measurements: Hall A Experiment E89-003 at Jefferson Laboratory, JLAB Internal Report (unpublished).

[35] A. Ketikyan, H. Voskanyan, B. Wojtsekhowski, About Shower Detector Software, JLAB Internal Report (unpublished).

[36] A. T. Katramatou et al., Nucl. Instr. Meth. A267, 448 (1988); and references therein.

[37] K. L. Brown et al., SLAC Report \# 91 (1977, unpublished).

[38] L. W. Mo and Y. S. Tsai, Rev. Mod. Phys. 41, 205 (1969); Y. S. Tsai, Radiative Corrections to Electron Scattering, SLAC-PUB-848 (1971).

[39] P. E. Bosted, Phys. Rev. C51, 409 (1995).

[40] M. Gari and H. Hyuga, Nucl. Phys. A264, 409 (1976). 
[41] E. L. Loman, Ann. Physics 125, 309 (1980).

[42] R. B. Wiringa, V.G.J. Stoks and R. Schiavilla, Phys. Rev. C. 51, 38 (1995).

[43] G. Höhler et al., Nucl. Phys. B114, 505 (1976).

[44] R. Schiavilla, V. R. Pandharipande and D. O. Riska, Phys. Rev. C 40, 2294 (1989).

[45] R. Schiavilla and D. O. Riska, Phys. Rev. C 43, 437 (1991).

[46] F. Gross, JLAB Report No. JLAB-THY-98-37 (unpublished)

[47] B. D. Keister and W. N. Polyzou, Adv. Nucl. Phys. 20, 225 (1991).

[48] E. E. Salpeter and H. A. Bethe, Phys. Rev. 84, 1232 (1951).

[49] F. Gross, Phys. Rev. 186, 1448 (1969).

[50] R. Blankenbecler and R. Sugar, Phys. Rev. 142, 1051 (1966).

[51] J. W. Van Orden, N. Devine and F. Gross, Phys. Rev. Lett. 75, 4369 (1995).

[52] F. Gross, J. W. Van Orden and K. Holinde, Phys. Rev. C. 45, 2094 (1992).

[53] Y. Surya and F. Gross, College of William and Mary Report No. WM-95-101; CEBAF Report No. CEBAF-TH-95-04.

[54] S. Galster et al., Nucl. Phys. B32, 221 (1971).

[55] E. Hummel and J. A. Tjon, Phys. Rev. C 42, 423 (1990); and references therein.

[56] M. J. Zuilhof and J. A. Tjon, Phys. Rev. C. 22, 2369 (1980).

[57] D. Berg et al., Phys. Rev. Lett. 44, 706 (1980).

[58] M. Chemtob, E. Moniz and M. Rho, Phys. Rev. C 10, 344 (1974).

[59] S. J. Brodsky and G. R. Farrar, Phys. Rev. Lett. 31, 1153 (1973). 
[60] V. A. Matveev, R. M. Muradyan and A. N. Tavkhelidze, Lett. Nuovo Cimento 7, 719 (1973).

[61] C. E. Carlson, J. R. Hiller, R. J. Holt, Annu. Rev. Nucl. Part. Sci. 47, 395 (1997).

[62] S. J. Brodsky, C-R. Ji and G. P. Lepage, Phys. Rev. Lett. 51, 83 (1983).

[63] C. E. Carlson and F. Gross, Phys. Rev. Lett. 53, 127 (1984).

[64] N. Isgur and C. H. Llewellyn Smith, Phys. Rev. Lett. 52, 1080 (1984); Phys. Lett. B 217, 535 (1989).

[65] G. R. Farrar, K. Huleihel, and H. Zhang, Phys. Rev. Lett. 74, 650 (1995). 


\section{Vita}

Kathleen Rae McCormick

Department of PHYSICS

Old Dominion University

Norfolk, VA 23529

\section{EDUCATION}

Ph.D., Hadronic Physics, August 1999

Old Dominion University

Dissertation: Measurement of the Deuteron Elastic Structure Functions at Large Momentum Transfers

M.S., Nuclear and Particle Physics, January 1995

University of Illinois at Urbana-Champaign

B.S., Physics, May 1993

Eastern Kentucky University

Honors Thesis: Reaction $Q$ Values from $(p, n \gamma)$ Thresholds

Typeset using $\mathrm{AT}_{\mathrm{EX}}$. 